\title{
LOW FREQUENCY ELECTROSTATIC INSTABILITIES \\ IN A TWO-DUST COMPONENT PLASMA
}

\author{
A DISSERTATION \\ SUBMITTED TO THE DEPARTMENT OF PHYSICS, \\ UNIVERSITY OF DURBAN-WESTVILLE \\ IN PARTIAL FULFILLMENT OF THE REQUIREMENTS \\ FOR THE DEGREE OF \\ MASTER OF SCIENCE
}

By

Shimul Kumar Maharaj

April 1999 


\section{Preface}

The work described in this thesis was carried out by the author in the Department of Physics, University of Durban-Westville, from January 1997 to January 1999, under the supervision of Professor R. Bharuthram.

These studies represent original work by the author and have not been submitted in any form to another university. Where use is made of the work of others, it has been duly acknowledged in the text. 


\section{Acknowledgements}

I wish to the thank the following people who contributed towards this thesis in various ways:

Professor R. Bharuthram, my supervisor, for his guidance, interest and availability.

My close colleagues Sadha, Juggy, Suleman and Shobana for their valued support. To Hu Edwards for assistance with the $\mathrm{LAT}_{\mathrm{E}} \mathrm{X}$ typesetting program. To Suri for the encouragement and the many cups of tea during the rounding up stages of this work. To Dr. Jetendra Parashar for the many useful discussions pertaining to life in general and the work contained herein.

The Brijlals of Westmeath Avenue for providing me with an extremely warm, comfortable and supportive home environment for the period in which this work was conducted. To Surajh, Nirisha and Reshmie for the funfilled evenings at the movies. To my mother, my pillar of support, and whose patience, motivation and steadfast guidance will continue to inspire me over the many years to come. To Akshay, Helena and my grandmother for the concern and the support.

To all the others whose encouragement and support is sincerely appreciated.

iii 


\section{Abstract}

The kinetic dispersion relation for a magnetized dusty plasma comprising of ions, electrons and massive, charged dust particles is solved for low frequency electrostatic instabilities in the dust plasma frequency regime. The free energy is provided by the drifting ion beam. The effect of varying parameters such as ion drift speed, particle densities, ion temperature and magnetic field strength on the real frequency and growth rate is examined. Initially light and heavy dust species of different charge are separately considered. This procedure is then repeated for a four-component plasma in an attempt to study the effect of the presence of both the dust species on low frequency electrostatic phenomena. Using a different plasma model, instabilities generated by an equal $\mathbf{E} \times \mathbf{B}$ drift of both the magnetized ions and electrons relative to the unmagnetized dust grains of both the heavy and light dust species is also investigated. The latter instabilities are applicable to the planetary ring plasmas of Saturn. Throughout our studies, numerical solutions of the full dispersion relation for the real frequency and growth rate are compared with approximate analytical solutions. 
To my loving mother, Akshay and in memory of my late father 


\title{
List of commonly used symbols
}

\author{
$\alpha_{j}$ \\ $=k_{\perp}^{2} C_{j}^{2} / \Omega_{j}^{2}$ (where $j=i, e, d l$ and $d h$ for the ions, electrons, light \\ and heavy dust particles respectively) \\ $\vec{B}_{o}\left(\mathbf{B}_{\mathbf{o}}\right)$ \\ external magnetic field in the $\hat{\mathbf{z}}$ direction \\ $C_{j}$ \\ $=\left(T_{j} / m_{j}\right)^{1 / 2}=$ thermal velocity of the $j^{\text {th }}$ species \\ $=\left(T_{\perp} / m_{i}\right)^{1 / 2}=$ thermal velocity of beam ions perpendicular to $\vec{B}_{o}$ \\ $=\left(T_{\|} / m_{i}\right)^{1 / 2}=$ thermal velocity of beam ions along $\vec{B}_{o}$ \\ $C_{s}$ \\ $=\left(T_{e} / m_{i}\right)^{1 / 2}=$ ion sound speed \\ $\delta$ \\ $n_{i o} / n_{e o}$ \\ magnitude of the electron charge \\ permittivity of free space \\ $\epsilon_{o}$ \\ distribution function of particles of type $j$ \\ $f_{j}$ \\ $f_{j o}$ \\ equilibrium distribution function of particles of type $j$ \\ $\Gamma_{p j}$ \\ $=e^{-\alpha_{j}} I_{\mathrm{p}}\left(\alpha_{j}\right)$ \\ i \\ $\sqrt{-1}$ \\ $I_{\mathrm{p}}$ \\ modified Bessel function of order $\mathrm{p}$ \\ $J_{\mathrm{p}}$ \\ Bessel function of order $\mathrm{p}$ \\ $\vec{k}$ \\ $=\left(k_{x}, k_{y}, k_{z}\right)=$ wave propagation vector
}




\begin{tabular}{|c|c|}
\hline$k_{\perp}$ & $=\left(k_{x}^{2}+k_{y}^{2}\right)^{1 / 2}=$ component of $\vec{k}$ perpendicular to $\vec{B}_{o}$ \\
\hline$k_{\|}$ & $=k_{z}=$ component of $\vec{k}$ along $\vec{B}_{o}$ \\
\hline$\lambda_{d}$ & $=\left(T_{e} / 4 \pi n_{o} e^{2}\right)^{1 / 2}=$ normalizing length \\
\hline \multirow[t]{3}{*}{$\lambda_{d j}$} & $=\left(T_{j} / 4 \pi n_{j o} q_{j}^{2}\right)^{1 / 2}=$ Debye length of $j^{t h}$ species \\
\hline & where $q_{j}=e,-e,-Z_{d h} e$ and $-Z_{d l} e$ (for $j=i, e, d h$ and $d l$ \\
\hline & respectively) \\
\hline$m_{j}$ & mass of particles of species $j(j=i, e, d l$ and $d h)$ \\
\hline$n_{j}$ & density of particles of species $j$ (for $j=e, i, d l$ and $d h$ ) \\
\hline$n_{j o}$ & equilibrium density of particles of species $j$ \\
\hline$n_{\circ}$ & total plasma density \\
\hline$\omega$ & $=\omega_{r}+\mathrm{i} \gamma=$ complex frequency \\
\hline $\bar{\omega}$ & $=\omega-\vec{k} \cdot \vec{V}_{o}=$ Doppler shifted frequency \\
\hline$\omega_{r}$ & real frequency \\
\hline$\gamma$ & growth rate \\
\hline \multirow[t]{3}{*}{$\Omega_{j}\left(\omega_{c j}\right)$} & $=\left|q_{j} B_{o} / m_{j} c\right|=$ gyrofrequency of the $j^{t h}$ species \\
\hline & where $q_{j}=e,-e,-Z_{d h} e$ and $-Z_{d l} e($ for $j=i, e, d h$ and $d l$ \\
\hline & respectively) \\
\hline \multirow[t]{3}{*}{$\omega_{p j}$} & $=\left(4 \pi n_{j o} q_{j}^{2} / m_{j}\right)^{1 / 2}=$ plasma frequency of species $j$ \\
\hline & where $q_{j}=e,-e,-Z_{d h} e$ and $-Z_{d l} e$ (for $j=i, e, d h$ and $d l$ \\
\hline & respectively) \\
\hline$\phi$ & electrostatic potential \\
\hline$\psi$ & angle between $k_{x}$ and $k_{\perp}$ \\
\hline$\theta$ & angle between $\vec{k}$ and $k_{x}$ \\
\hline
\end{tabular}


$\Theta$

$T_{\perp}$

$T_{\|}$

$T_{j}$

$\vec{V}$

$\sigma$

$\beta$

$V_{\perp}$

$\vec{V}_{E}$

$\vec{V}_{d i}\left(\vec{V}_{o}\right)$

$Z(\lambda)$

$Z_{j}$

$\nabla$ azimuthal angle

beam ion temperature perpendicular to $\vec{B}_{0}$

beam ion temperature along $\vec{B}_{o}$

temperature of particles of species $j(j=e, i, d l$ and $d h)$

velocity vector

$=T_{\perp} / T_{\|}$

$=1-\sigma$

$=\left(V_{x}^{2}+V_{y}^{2}\right)^{1 / 2}=$ component of $\vec{V}$ in $x-y$ plane

$\vec{E} \times \vec{B}_{o}$ drift of the ions and electrons (Chapter 6)

ion drift velocity

plasma dispersion function with argument $\lambda$

the number of electronic charges on each dust grain of the $j^{\text {th }}$ species $(j=d l$ and $d h)$

differential operator $\frac{\partial}{\partial x} \hat{x}+\frac{\partial}{\partial y} \hat{y}+\frac{\partial}{\partial z} \hat{z}$ 


\section{Contents}

Preface $\quad$ ii

Acknowledgements $\quad$ iii

Abstract iv

List of commonly used symbols vi

1 Introduction $\quad 1$

2 Literature Survey 5

3 Derivation of the Kinetic Dispersion Relation 12

3.1 Contribution of Background Species to Dispersion Relation . . . . . . 21

3.2 Approximate Solutions of the Kinetic Dispersion Relation . . . . . . . 23

4 Solution of the Dispersion Relation for a Single Dust Component $\begin{array}{ll}\text { Plasma } & \mathbf{3 4}\end{array}$

4.1 Heavy Dust Component . . . . . . . . . . . . . . . 34

4.1 .1 Numerical Results . . . . . . . . . . . . . 35 
4.2 Light Dust Component . . . . . . . . . . . . . . 51

4.2 .1 Numerical Results ... . . . . . . . . . . . 51

5 Solution of the Dispersion Relation for a Four-Component Plasma (Two Dust Species)

5.1 Numerical Results . . . . . . . . . . . . . . . . 65

6 Solution of the Dispersion Relation for Equal Crossfield Drift of

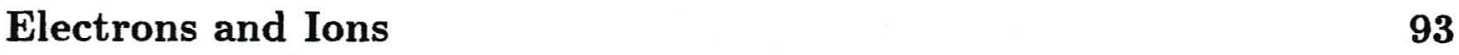

6.1 The Kinetic Dispersion Relation . . . . . . . . . . . . . . . . . 94

6.2 Numerical Results . . . . . . . . . . . . . . . . 95

$\begin{array}{ll}7 \text { Conclusion } & 104\end{array}$

8 References 108 


\section{List of Figures}

4.1 Plot of normalized growth rate against normalized wavenumber. The parameter labelling the curves is the normalized ion drift speed $V_{d i z} / C_{s}$. The fixed parameters are $T_{i}=T_{e}, T_{d h}=0.01 T_{e}, n_{e o}=0.1 n_{o}, m_{d h} / m_{i}=$

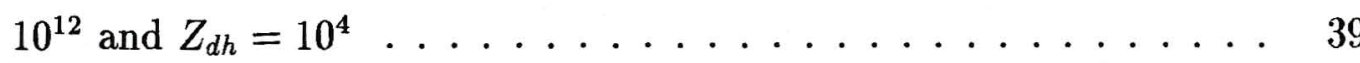

4.2 Plot of the normalized real frequency versus normalized wavenumber for $V_{d i z}=0.3 C_{s}$. The broken line shows the real frequency calculated from the approximate expression (4.3). All other parameters are as in Figure $4.1 \ldots \ldots \ldots \ldots \ldots \ldots \ldots \ldots$

4.3 Plot of normalized growth rate against normalized wavenumber for $V_{d i z}=0.3 C_{s}$. All other fixed parameters are as in Figure 4.1. The broken line shows the growth rate calculated from the approximate expression $(4.5) \ldots \ldots \ldots \ldots \ldots \ldots \ldots$

4.4 Plot of the normalized growth rate against normalized wavenumber for $V_{d i z}=0.2 C_{s}$. The parameter labelling the curves is the normalized electron density $n_{e o} / n_{o}$. All other fixed parameters are as in Figure 4.143 
4.5 Plot of normalized real frequencies corresponding to the growth rate curves in Figure 4.4. The parameter labelling the curves is the normalized equilibrium electron density $n_{e o} / n_{o} \ldots \ldots \ldots$

4.6 Plot of normalized growth rate against normalized wavenumber for $V_{d i z}=0.2 C_{s}$. The parameter labelling the curves is the normalized ion temperature $T_{i} / T_{e}$. All other fixed parameters are as in Figure 4.1

4.7 Plot of the normalized real frequencies corresponding to the curves in Figure 4.6. The parameter labelling the curves is the normalized ion temperature $T_{i} / T_{e} \ldots \ldots \ldots \ldots \ldots$

4.8 Plot of the normalized growth rates as a function of normalized wave number for different values of the electron density $n_{e o} / n_{o}$ : (a) $n_{e o}=$ $0.00 n_{o}$, (b) $n_{e o}=0.5 n_{o}$, (c) $n_{e o}=0.75 n_{o}$ and (d) $n_{e o}=0.95 n_{o}$. The parameter labelling the curves is $T_{i} / T_{e}$. All other parameters are as in Figure $4.6 \ldots \ldots \ldots \ldots \ldots \ldots \ldots \ldots$

4.9 Plot of normalized growth rate as a function of $k_{y} / k$. The parameter labelling the curves is $\omega_{p i} / \Omega_{i}$. Fixed parameters are $V_{d i z}=0.2 C_{s}, k \lambda_{d}=$ 2.4 and $T_{i}=0.1 T_{e}$. All other parameters are as in Figure $4.1 \ldots 47$

4.10 Plot of the normalized real frequency (1) and growth rate (2) versus $k \lambda_{d}$ for oblique angles of propagation relative to $\mathbf{B}_{\mathbf{o}}$. Here $T_{i}=T_{e}$ and $k_{y} / k=0.707\left(\theta=45^{\circ}\right)$ for various values of $\omega_{p i} / \Omega_{i}$ : (a) $\omega_{p i} / \Omega_{i}=0.01$, (b) $\omega_{p i} / \Omega_{i}=0.5$ and (c) $\omega_{p i} / \Omega_{i}=1.0$. All other fixed parameters are as in Figure $4.9 \ldots \ldots \ldots \ldots \ldots \ldots$ 
4.11 Plot of the normalized real frequency (1) and growth rate (2) versus $k \lambda_{d}$. Here $k_{y} / k=0.5\left(\theta=30^{\circ}\right)$ for various values of $\omega_{p i} / \Omega_{i}$ : (a) $\omega_{p i} / \Omega_{i}=0.01$, (b) $\omega_{p i} / \Omega_{i}=0.5$ and (c) $\omega_{p i} / \Omega_{i}=1.0$. All other fixed parameters are as in Figure $4.10 \ldots \ldots \ldots \ldots$

4.12 Plot of normalized real frequency (1) and growth rate (2) versus $k \lambda_{d}$. Here $k_{y} / k=0.95\left(\theta=72^{\circ}\right)$ for various values of $\omega_{p i} / \Omega_{i}:$ (a) $\omega_{p i} / \Omega_{i}=$ 0.01 , (b) $\omega_{p i} / \Omega_{i}=0.5$ and (c) $\omega_{p i} / \Omega_{i}=1.0$. All other fixed parameters are as in Figure $4.10 \ldots \ldots \ldots \ldots$

4.13 Plot of the normalized growth rate against normalized wavenumber. The parameter labelling the curves is the normalized ion drift speed $V_{d i z} / C_{s}$. The fixed parameters are $T_{i}=T_{e}, T_{d l}=0.01 T_{e}, n_{e o}=$ $0.1 n_{o}, m_{d l} / m_{i}=10^{6}$ and $Z_{d l}=10^{2} \ldots \ldots \ldots \ldots$

4.14 Plot of normalized growth rate against normalized wavenumber for $V_{d i z}=0.2 C_{s}$. The parameter labelling the curves is the normalized electron density $n_{e o} / n_{o}$. All other fixed parameters are as in Figure 4.1355

4.15 Plot of normalized real frequencies corresponding to the growth rate curves in Figure 4.14. The parameter labelling the curves is $n_{e o} / n_{o}$.

4.16 Plot of the normalized growth rate against normalized wavenumber for $V_{d i z}=0.2 C_{s}$. The parameter labelling the curves is the normalized ion temperature $T_{i} / T_{e}$. All other fixed parameters are as in Figure 4.1356

4.17 Plot of normalized real frequencies corresponding to the growth rate curves in Figure 4.16. The parameter labelling the curves is $T_{i} / T_{e} \ldots 56$ 
4.18 Plot of normalized growth rate versus $k \lambda_{d}$ for different values of the equilibrium electron density $n_{e o} / n_{o}:$ (a) $n_{e o}=0.00 n_{o}$, (b) $n_{e o}=0.5 n_{o}$, (c) $n_{e o}=0.75 n_{o}$ and (d) $n_{e o}=0.95 n_{o}$. The parameter labelling the curves is $T_{i} / T_{e}$. All other parameters are as in Figure $4.1 \ldots 57$

4.19 Plot of normalized growth rate as a function of $k_{y} / k$. The parameter labelling the curves is $\omega_{p i} / \Omega_{i}$. Fixed parameters are $V_{d i z}=0.2 C_{s}, k \lambda_{d}=$ 2.4 and $T_{i}=0.1 T_{e}$. All other parameters are as in Figure $4.13 \ldots$.

4.20 Plot of normalized real frequency (1) and growth rate (2) versus $k \lambda_{d}$ for oblique angles of propagation relative to $\mathbf{B}_{\mathbf{0}}$. Here $T_{i}=T_{e}$ for (a) $k_{y} / k=0.707\left(\theta=45^{\circ}\right)$ and (b) $k_{y} / k=0.5\left(\theta=30^{\circ}\right)$. The parameter labelling the curves is $\omega_{p i} / \Omega_{i}$. All other fixed parameters are as in Figure $4.19 \ldots \ldots \ldots \ldots \ldots \ldots \ldots \ldots$

4.21 Plot of the normalized real frequency (1) and growth rate (2) versus $k \lambda_{d}$ for $k_{y} / k=0.95\left(\theta=72^{\circ}\right)$. Here $\omega_{p i} / \Omega_{i}=0.01$. All other fixed parameters are as in Figure $4.20 \ldots \ldots 6$

4.22 Plot of the normalized real frequency (1) and growth rate (2) versus $k \lambda_{d}$ for $k_{y} / k=0.95\left(\theta=72^{\circ}\right)$. Here $\omega_{p i} / \Omega_{i}=0.5$. All other fixed parameters are as in Figure $4.20 \ldots \ldots \ldots \ldots$

4.23 Plot of the normalized real frequency (1) and growth rate (2) versus $k \lambda_{d}$ for $k_{y} / k=0.95\left(\theta=72^{\circ}\right)$ and $\omega_{p i} / \Omega_{i}=1.0$. All other fixed parameters are as in Figure $4.20 \ldots \ldots \ldots \ldots$ 
5.1 Plot of normalized growth rate versus normalized wavenumber. The parameter labelling curves is the normalized ion drift speed $V_{d i z} / C_{s}$. The fixed parameters are $T_{i}=T_{e}, T_{d l}=0.01 T_{e}, T_{d h}=0.01 T_{e}, n_{e o}=$ $0.1 n_{o}, n_{d l o}=n_{d h o}, m_{d l} / m_{i}=10^{6}, Z_{d l}=10^{2}, m_{d h} / m_{i}=10^{12}$ and $Z_{d h}=$ $10^{4} \ldots \ldots \ldots \ldots \ldots \ldots \ldots \ldots \ldots \ldots \ldots \ldots \ldots$

5.2 Plot of the normalized real frequency against normalized wavenumber for $V_{d i z}=0.3 C_{s}$. The dashed curve shows the result using the approximate analytic expression (5.3). All other fixed parameters are as in

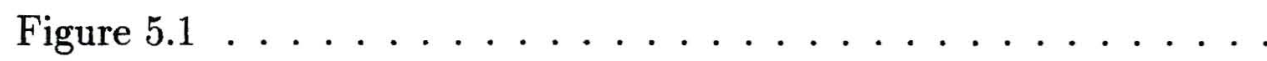

5.3 Plot of the normalized growth rate as a function of normalized wave number for $V_{d i z}=0.3 C_{s}$. The dashed curve shows the result calculated from the approximate expression (5.4). All other parameters are as in

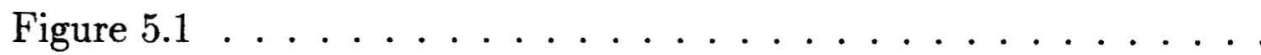

5.4 Plot of the normalized real frequency versus $n_{d l o} / n_{d h o}$ for $V_{d i z}=0.2 C_{s}$. Here $k \lambda_{d}=0.7$. All other fixed parameters are as in Figure 5.1 . . .

5.5 Plot of the normalized growth rate corresponding to the real frequency curve of Figure $5.4 \ldots \ldots \ldots \ldots \ldots$

5.6 Plot of the analytical solutions of real frequency versus normalized wave number for the three-component plasmas corresponding to the light and heavy dust constituents and for a four-component (combined light and heavy dust species) plasma. All fixed parameters are as in Figure $5.1 \ldots \ldots \ldots \ldots \ldots \ldots \ldots$ 
5.7 Plot of the normalized growth rate versus normalized wave number for $V_{d i z}=0.2 C_{s}$. The parameter labelling the curves is the normalized electron density $n_{e o} / n_{o}$. All other fixed parameters are as in Figure 5.175

5.8 Plot of normalized real frequencies corresponding to the growth rates in Figure 5.7. The parameter labelling the curves is $n_{e o} / n_{o} \ldots \ldots$

5.9 Plot of the normalized growth rate versus $k \lambda_{d}$ for $V_{d i z}=0.2 C_{s}$ for (a) $n_{e o}=0.00 n_{o}$, (b) $n_{e o}=0.5 n_{o}$ and (c) $n_{e o}=0.75 n_{o}$. The parameter labelling the curves is the normalized ion temperature $T_{i} / T_{e}$. All other fixed parameters are as in Figure $5.1 \ldots \ldots \ldots$

5.10 Plot of the normalized real frequency (1) and growth rate (2) versus $n_{e o} / n_{o}$ for (a) $k \lambda_{d}=1.0$ and (b) $k \lambda_{d}=2.0$. The parameter labelling the curves is $T_{i} / T_{e}$. All other fixed parameters are as in Figure 5.1 .

5.11 Plot of the normalized growth rate versus $k \lambda_{d}$ for $V_{d i z}=0.2 C_{s}$. The parameter labelling the curves is $T_{i} / T_{e}$. All other fixed parameters are as in Figure $5.1 \ldots \ldots \ldots \ldots \ldots$

5.12 Plot of normalized real frequencies corresponding to the growth rates of Figure 5.11. The parameter labelling the curves is $T_{i} / T_{e} \ldots \ldots$

5.13 Plot of the normalized real frequency versus $k \lambda_{d}$ for $V_{d i z}=0.2 C_{s}$. The parameter labelling the curves is $T_{i} / T_{e}$. The dashed curves are calculated from the approximate expression (5.3). All other fixed parameters are as in Figure $5.1 \ldots \ldots \ldots$. . . . . . . . 80 
5.14 Plot of normalized real frequency as a function of $k \lambda_{d}$ for $V_{d i z}=0.2 C_{s}$. The parameter labelling the curves is the normalized electron density $n_{e o} / n_{o}$. Here $T_{i}=T_{e}$. The dashed curves are calculated from the approximate expression (5.3). All other fixed parameters are as in Figure $5.1 \ldots \ldots \ldots \ldots \ldots \ldots$

5.15 Plot of the normalized growth rate versus $k_{y} / k$ for $V_{d i z}=0.2 C_{s}$. Here $T_{i}=0.1 T_{e}$ and $k \lambda_{d}=2.4$. The parameter labelling the curves is $\omega_{p i} / \Omega_{i}$. All other fixed parameters are as in Figure $5.1 \ldots \ldots$

5.16 Plot of normalized real frequency (1) and growth rate (2) versus $k \lambda_{d}$ for oblique angles of propagation relative to $\mathbf{B}_{\mathbf{o}}$. Here $T_{i}=T_{e}, k_{y} / k=$ $0.707\left(\theta=45^{\circ}\right)$ and $\omega_{p i} / \Omega_{i}=0.01$. All other fixed parameters are as in Figure $5.15 \ldots \ldots \ldots \ldots \ldots \ldots \ldots$

5.17 Plot of normalized real frequency (1) and growth rate (2) versus $k \lambda_{d}$ for $k_{y} / k=0.707\left(\theta=45^{\circ}\right)$. Here $\omega_{p i} / \Omega_{i}=0.5$. All other fixed parameters are as in Figure $5.15 \ldots \ldots \ldots \ldots \ldots$

5.18 Plot of normalized real frequency (1) and growth rate (2) versus $k \lambda_{d}$ for $k_{y} / k=0.707\left(\theta=45^{\circ}\right)$. Here $\omega_{p i} / \Omega_{i}=1.0$. All other fixed parameters are as in Figure $5.15 \ldots \ldots \ldots \ldots \ldots$

5.19 Plot of normalized real frequency (1) and growth rate (2) versus $k \lambda_{d}$ for oblique angles of propagation relative to $\mathbf{B}_{\mathbf{o}}$. Here $T_{i}=T_{e}, k_{y} / k=0.5$ $\left(\theta=30^{\circ}\right)$ and $\omega_{p i} / \Omega_{i}=0.01$. All other fixed parameters are as in Figure 5.15 
5.20 Plot of normalized real frequency (1) and growth rate (2) versus $k \lambda_{d}$ for $k_{y} / k=0.5\left(\theta=30^{\circ}\right)$. Here $\omega_{p i} / \Omega_{i}=0.5$. All other fixed parameters are as in Figure $5.15 \ldots \ldots \ldots \ldots \ldots$

5.21 Plot of normalized real frequency (1) and growth rate (2) versus $k \lambda_{d}$ for $k_{y} / k=0.5\left(\theta=30^{\circ}\right)$. Here $\omega_{p i} / \Omega_{i}=1.0$. All other fixed parameters are as in Figure $5.15 \ldots \ldots \ldots \ldots \ldots$

5.22 Plot of normalized real frequency (1) and growth rate (2) versus $k \lambda_{d}$ for oblique angles of propagation relative to $\mathbf{B}_{\mathbf{o}}$. Here $T_{i}=T_{e}, k_{y} / k=0.95$ $\left(\theta=72^{\circ}\right)$ and $\omega_{p i} / \Omega_{i}=0.01$. All other fixed parameters are as in Figure $5.15 \ldots \ldots \ldots \ldots \ldots \ldots \ldots$

5.23 Plot of normalized real frequency (1) and growth rate (2) versus $k \lambda_{d}$ for $k_{y} / k=0.95\left(\theta=72^{\circ}\right)$. Here $\omega_{p i} / \Omega_{i}=0.5$. All other fixed parameters are as in Figure $5.15 \ldots \ldots \ldots \ldots \ldots$

5.24 Plot of normalized real frequency (1) and growth rate (2) versus $k \lambda_{d}$ for $k_{y} / k=0.95\left(\theta=72^{\circ}\right)$. Here $\omega_{p i} / \Omega_{i}=1.0$. All other fixed parameters are as in Figure $5.15 \ldots \ldots \ldots \ldots$

5.25 Plot of normalized real frequencies as a function of $k \lambda_{d}$ for the two modes in Figure 5.20. The dashed curve corresponds to the approximate solution (5.3). All other fixed parameters are as in Figure 5.20 .

5.26 Plot of normalized growth rates corresponding to the real frequency curves for the two modes in Figure $5.25 \ldots \ldots \ldots \ldots$ 
6.1 Plot of normalized growth rate versus $k_{y} / k(\sin \theta)$. The parameter labelling the curves is the normalized equal ion and electron drift speed $V_{E} / C_{s}$. The fixed parameters are $T_{i}=T_{e}, T_{d l}=0.01 T_{e}, T_{d h}=$ $0.01 T_{e}, n_{e o}=0.1 n_{o}, n_{d l o}=n_{d h o}, Z_{d l}=10^{2}, m_{d l}=10^{6} m_{i}, Z_{d h}=$ $10^{4}, m_{d h}=10^{12} m_{i}, k \lambda_{d}=0.7$ and $\omega_{p i}=0.01 \omega_{c i} \ldots \ldots 96$

6.2 Plot of normalized real frequencies corresponding to the growth rate curves of Figure 6.1. The parameter labelling the curves is $V_{E} / C_{s} \ldots 96$

6.3 Plot of the normalized real frequency and growth rate versus $k \lambda_{d}$ for (a) $k_{y} / k=0.175\left(\theta=10^{\circ}\right)$ and (b) $k_{y} / k=0.993\left(\theta=83^{\circ}\right)$. Here $V_{E}=$ $10 C_{s}$. The broken line is the result calculated from the approximate solution (6.2). All other fixed parameters are as in Figure 6.1 . . . .

6.4 Plot of the normalized real frequency and growth rate versus $k \lambda_{d}$ for (a) $k_{y} / k=0.175\left(\theta=10^{\circ}\right)$ and (b) $k_{y} / k=0.993\left(\theta=83^{\circ}\right)$. Here $m_{d}=10^{12} m_{i}$ and $Z_{d}=10^{4}$ for a three-component (single heavy dust species) plasma. The broken line is the result calculated from the approximate solution (6.3). All other fixed parameters are as in Figure

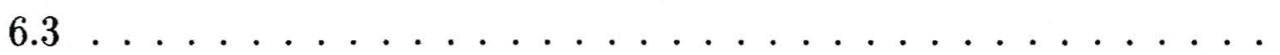

6.5 Plot of the normalized growth rate versus $k_{y} / k$ for $V_{E}=10 C_{s}$. The parameter labelling the curves is the normalized equilibrium electron density $n_{e o} / n_{o}$. All other fixed parameters are as in Figure 6.1 . . 100

6.6 Plot of normalized growth rate against $k_{y} / k$. The parameter labelling the curves is the normalized ion temperature $T_{i} / T_{e}$. All other parameters are as in Figure $6.5 \ldots \ldots \ldots 2$ 
6.7 Plot of the normalized growth rate as a function of $k_{y} / k$. Here $n_{e o}=$ $0.1 n_{o}$. The parameter labelling the curves is $n_{d l o} / n_{d h o}$. All other fixed parameters are as in Figure $6.5 \ldots \ldots . \ldots . \ldots . \ldots 103$

6.8 Plot of normalized real frequencies corresponding to the growth rate curves in Figure 6.7. The parameter labelling the curves is $n_{d l o} / n_{d h o} \cdot 103$ 


\section{Chapter 1}

\section{Introduction}

The concept of 'plasma instability' has attained widespread attention because of its importance in nuclear fusion and space research. Plasma instabilities play a vital role in many phenomena such as magnetic reconnection, and solar and pulsar radiation which occur in space and astrophysical plasmas. These instabilities are also detrimental to the confinement of a thermonuclear plasma. Instabilities arise because plasmas are usually far from their equilibrium states and hence contain certain amounts of free energy. The natural tendency for a plasma is to get rid of some of its excess energy and ultimately attain a state of thermodynamic equilibrium. It does this by shedding this energy to some of its normal wave modes and hence causing their amplitudes to grow in time. Such a growing plasma mode is referred to as being an unstable mode. Plasma instability refers to this process whereby the free energy in a plasma becomes converted into a growing wave mode.

The interest in studying linear and nonlinear instabilities in the so called "dusty" plasmas is fairly recent, this being motivated by the fact that charged dust has been 
increasingly found in cometary environments, planetary rings, interstellar clouds and also in laboratory devices. These dust grains are extremely variable with regard to shape, size, mass and charge. The dust grains range from micron, submicron sized grains to extremely large boulders. Grain charge is not fixed but depends on the properties of the surrounding plasma, photo-ionization processes, secondary electron emission and ultra-violet ray irradiation. If charging effects other than those due to the surrounding plasma are neglected, the dust grain must aquire a negative charge (as the more mobile electrons reach the grain surface first) in order to equalize the ion and electron thermal fluxes to the grain surface, and attain a state of equilibrium. Most theoretical treatments, therefore, assume the dust grains to be negatively charged. However, other processes such as photo-ionization, secondary electron emission and ultra-violet ray irradiation may result in grains becoming positively charged. Once the dust is charged it starts to react to electromagnetic as well as to gravitational forces. One finds that a grain must be in the micrometre range of sizes before electromagnetic effects start becoming dominant over gravitational effects (Northrop,1992).

The three fundamental length scales for a combined dust-plasma mixture are the grain size $a$, the Debye length $\lambda_{D}$ and an average intergrain distance $d$ which is roughly related to the dust density by $d \approx n_{d}^{-1 / 3}$. The grain size $a$ is the smallest of the three lengths. Single particle dynamics dominate over collective effects for $a \ll \lambda_{D}<d$. On the other hand, collective effects become important when $a \ll d<\lambda_{D}$. It is this latter case that we refer to as being a dusty plasma.

Dusty plasmas are similar to plasmas comprising of ions and electrons except that 
the dust grains have large mass and charge. Each dust grain can be highly negatively charged with up to $10^{4}$ electrons on a single dust grain. The mass of a typical dust grain is about $10^{6}-10^{12}$ that of the proton. The time scales associated with dust phenomena will therefore be orders of magnitude greater than those associated with the ion component of the plasma. The entire spectrum of plasma waves will therefore be modified by the presence of the charged dust component. Rao et al. (1990) found that a new 'sound' wave called a dust-acoustic wave could propagate in such a plasma. Linear dust-acoustic waves have been detected very recently in a laboratory experiment by Barkan et al. [2]. The low frequency density fluctuations of dust particles, which seems to be consistent with the theory of dust-acoustic waves, was observed by Prabhakara et al. (1996) in a hot cathode discharge device. Dust grains are also known to have been grown in laboratory plasmas to form the so called dust 'plasma crystals' which are Coulomb lattices of charged dust grains (Thomas et al.,1994).

The linear theory of waves in a dusty plasma is well developed. Wave excitation mechanisms may also give rise to large amplitude waves where nonlinear effects become important such as leading to the formation of solitary wave structures (solitons). Dusty plasmas encountered in the laboratory as well as in space environments are often immersed in an external magnetic field, therefore it is also imperative to study the effects of a magnetic field on wave phenomena in plasmas.

This thesis presents a study of low frequency electrostatic instabilities in a magnetized dusty plasma. A brief outline of the thesis is as follows: 
A survey of relevant literature is presented in Chapter 2. In Chapter 3 we derive the relevant dispersion relation and present approximate analytical solutions.

Chapter 4 investigates the dust-acoustic instability in a three-component plasma consisting of drifting ions, stationary electrons and a single, stationary heavy dust component. The ion drift is parallel to the external magnetic field. The effect of the variation of parameters such as ion drift speed, densities, ion temperature and magnetic field strength on the instability real frequency and growth rate is studied. The effect of the presence of a single light dust component on the dust-acoustic instability in a three-component plasma is also studied.

Chapter 5 examines the effect of two different species of dust (of different mass and charge) on the dust-acoustic instability in a four-component plasma. A complete variation of parameters as done in the previous chapter is undertaken.

Chapter 6 serves to examine the transition from the dust-acoustic instability to the dust-modified two-stream instability in a four-component plasma comprising of drifting ions, drifting electrons and two stationary dust components of equal density. Mass and charge of both dust components are the same as in preceding chapters. These instabilities are generated by equal $\mathbf{E} \times \mathbf{B}_{\mathbf{o}}$ drifts of both the magnetized electrons and ions across the external magnetic field, while the dust grains are treated as being unmagnetized.

Finally in Chapter 7 we present a summary of our results. 


\section{Chapter 2}

\section{Literature Survey}

In this chapter we present a discussion of the reported works in the literature that are relevant to our investigations.

The first study of wave phenomena on the dust time-scale was made by Rao et al. (1990). They studied the linear and weakly nonlinear properties of the dust-acoustic wave. In their analysis, the motion of the dust particles was assumed to be described by the fluid equations. The electrons and the ions were assumed to be in thermodynamic equilibrium and their densities described by Boltzmann distributions, because of their relatively small mass in comparison to the dust grains. In the nonlinear limit, they found that dust-acoustic solitons of either positive or negative electrostatic potential could propagate in the plasma.

Using a standard Vlasov analysis, M. Rosenberg (1993) investigated the conditions for the excitation of dust ion-acoustic (DIA) and dust-acoustic (DA) instabilities driven by weak ion and/or electron drifts in an unmagnetized dusty plasma. It was found that the dust ion-acoustic mode could be be driven unstable by an electron drift even 
in an isothermal plasma with $T_{i}=T_{e}$, provided that the dust grains carry most of the negative charge $\left(n_{i} / n_{e}>>1\right)$. Approximate expressions for the real frequency and growth rates of both instabilities are presented. Possible applications to collapsing protostellar clouds and Saturn's rings are discussed.

Using a standard linear Vlasov analysis, V.W. Chow and M. Rosenberg (1995) investigated the effect of the presence of charged dust on the electrostatic ion cyclotron instability (EICI). Although the EIC wave propagates almost perpendicular to the external magnetic field $\mathbf{B}_{\mathbf{o}}$, the wave vector has a small but finite component along $\mathbf{B}_{\mathbf{o}}$. The EICI is driven by electrons drifting along the direction of the magnetic field. They found that for negatively charged dust grains as $\delta=n_{i o} / n_{e o}$ increases, the critical electron drift for the onset of the instability decreases and the instability is enhanced. On the other hand, the presence of positively charged dust grains tends to stabilize the EICI. A possible application to the environment linking Jupiter to its satellite Io is discussed.

M. Rosenberg and N. A. Krall (1995) studied modified two-stream instabilities in a three-component magnetized dusty plasma driven by electrons and/or ions drifting across an external magnetic field. Instabilities in both the high frequency $\left(\omega_{c i} \ll \omega \ll \omega_{c e}\right)$ and low frequency $\left(\omega_{c d} \ll \omega \ll \omega_{c i}\right)$ regimes are studied via the full dispersion relation. In the former case the instability is driven by a drift of the magnetized electrons relative to the unmagnetized ions and the unmagnetized dust grains. The low frequency instability is driven by a drift of both the magnetized electrons and the 
magnetized ions relative to the unmagnetized dust grains. Both numerical and approximate analytical solutions of the dispersion relation are presented. Applications to dusty protostellar plasmas and planetary rings are also mentioned.

Using a kinetic approach, R. Bharuthram and T. Pather (1996) studied the dustacoustic instability in a three-component magnetized plasma consisting of stationary electrons, drifting ions and a stationary, massive, negatively charged dust component. The instability is driven by the energy provided by the drift of the ions along the magnetic field direction. Both numerical and approximate analytical solutions of the kinetic dispersion relation are presented. They found that

(1) The instability growth rates $\gamma$ increase with an increase in the drift speed of the ions. For normalized beam speeds $V_{d i z} / C_{s}<0.01$, the Landau damping due to the electrons and the negatively charged dust component is strong and there is no growth. (2) Due to the kinetic nature of the dust-acoustic instability, the growth rate $\gamma$ decreases as the normalized ion temperature $T_{i} / T_{e}$ increases.

(3) For a fixed $k \lambda_{d}$, the real frequency decreases as the normalized equilibrium electron density $n_{e o} / n_{o}$ increases. This behaviour agrees well with the analytical expression of the real frequency.

(4) The instability growth rates increase as $Z_{d}$ increases where $Z_{d}$ is the number of electronic charges on each dust grain. The growth rates also increase with a reduction in the dust grain to ion mass ratios.

(5) As the magnetization of the plasma increases, the range of propagation angles for the instability widens. 
The numerical results were found to be in excellent agreement with approximate analytical solutions of the dispersion relation.

R. Bharuthram (1997) examined low frequency electrostatic instabilities in a threecomponent dusty plasma driven by equal $\vec{E} \times \vec{B}$ drifts of both the ions and electrons across an external magnetic field. There is a transition from the dust-acoustic instability (DAI) for small angles of wave propagation to the dust-modified two-stream instability (DMTSI) of Rosenberg and Krall (1995) which becomes excited for wave propagation almost perpendicular to the magnetic field. A variation of the normalized ion temperature $T_{i} / T_{e}$ produces results that are consistent with the findings of Bharuthram and Pather (1996) for wave propagation in the direction of the magnetic field. The DAI was found to be more easily excited in plasmas having a high concentration of negatively charged, massive dust particles. The opposite was found to be true for the DMTSI. Results could be applicable to Saturn's rings where both the ions and electrons have an azimuthal drift relative to the levitated dust grains.

M. Rosenberg and V.W. Chow (1998) examined the effect of charged dust on the Farley-Buneman instability in a three component dusty plasma. The instability is applicable to the E-region of the ionosphere at altitudes of about $90-130 \mathrm{~km}$. In the presence of an electric field $\vec{E}_{o}$ perpendicular to $\vec{B}$, the instability is driven by a crossfield $\vec{E}_{o} \times \vec{B}$ drift of the electrons relative to the ions. In their analysis, they assumed that $\Omega_{e}=e B / m_{e} c \gg \nu_{e n}, \Omega_{i}=e B / m_{i} c \ll \nu_{i n}$ and $\Omega_{d}=Z_{d} e B / m_{d} c \ll \nu_{d n}$ where $\Omega_{e}\left(\Omega_{i}\right)\left(\Omega_{d}\right)$ is the electron (ion) (dust) gyrofrequency respectively and $\nu_{i n}$ $\left(\nu_{e n}\right)\left(\nu_{d n}\right)$ is the ion (electron) (dust)-neutral collision frequency. They found that 
the critical drift for excitation of the instability decreases as the charge density of the negatively charged dust grains increases, as was found for the EICI (Chow and Rosenberg,1995).

Most theoretical treatments of dusty plasmas assume the dust grains to be uniform with regard to shape, size, mass and charge. However in real dusty plasmas the size distribution of the dust grains must be considered. P. Meuris (1997) made a comparison of the dust plasma frequency of

(1) mono-sized dust grains

(2) dust grain size following a power law distribution with the differential dust density given by

$$
n(a) d a=K a^{-\beta} d a
$$

for charged dust grains with grain radii $a$ in the range $\left[a_{m i n} ; a_{m a x}\right]$. They found that the dust plasma frequency increases when a power law or a normal grain size distribution is considered. This correction is negligible for $\beta$ values greater than 2 but is significant for smaller values.

The focus of initial experimental work on dusty plasmas was on the measurement of charge on individual dust grains (Robertson,1995). It was not until very recently that experiments on collective processes in a plasma such as waves and instabilities were undertaken. Barkan et al. [1] investigated electrostatic ion cyclotron (EIC) waves in a Q-machine modified by the presence of a dust dispenser. They found that as the dust grains became increasingly negatively charged, larger amplitude waves were produced 
indicating that the EIC instability was enhanced. This behaviour is in good agreement with theoretical predictions. Linear dust-acoustic waves have been detected recently in a Q-machine by Barkan et al. [2]. Both theoretical and experimental studies of low frequency electrostatic waves in a magnetized dusty plasma were carried out by Merlino et al. (1998). The experiment was carried out in a dusty plasma device (DPD) which is a Q-machine modified by the presence of a rotating dust dispenser.

The plasma was created by ionization of potassium atoms supplied by an atomic beam. A dusty plasma was created by introducing kaolin (aluminium silicate) into the plasma column of the device. Further experiments on dust-acoustic waves were carried out in a glow discharge device containing nitrogen gas. The experiments performed confirm that the ion-acoustic and ion cyclotron instabilities are more easily excited in a dusty plasma consisting of negatively charged dust grains. Taking into account collision effects between the dust grains and the neutral gas molecules, the experimental results on the dust-acoustic mode were in good agreement with the theoretical dispersion relation obtained from fluid theory.

H.R. Prabhakara and V.L. Tanna (1996) investigated the trapping of negatively charged dust particles in a hot cathode filament plasma discharge device. The dust particles were made visible by the scattering of the light of a He-Ne laser. The source of the dust was about $5 \mathrm{~g}$ of alumina powder (average grain diameter $0.3 \mu \mathrm{m}$ ) placed in an aluminium plate at the bottom of the device. After aquiring a negative charge, it was observed that the dust particles became trapped in a plasma which was maintained at a positive potential with respect to the walls of the device. Due to the 
electric fields in the plasma being very weak, the dust grains began to fall under the influence of gravity, but would be pushed back into the plasma due to the repelling force of the electric field of a sheath region surrounding the lower plate. The observed low frequency density fluctuations of the dust was found to agree well with the theory of dust-acoustic waves although a more reliable method of measuring the number density of dust particles was required. 


\section{Chapter 3}

\section{Derivation of the Kinetic Dispersion Relation}

In our derivation of the kinetic dispersion relation we follow the method of Gary et al. (1970) and Singh (1989). As a general treatment, we consider a three-component collisionless plasma comprising of an anisotropic ion beam and stationary, isotropic background electron and dust components. A uniform magnetic field $\mathbf{B}_{\mathbf{o}}$ is assumed to be present in the $\hat{\mathbf{z}}$-direction. We assume that there is no equilibrium electric field $\left(\vec{E}_{o}=0\right)$. The electrostatic approximation is used, therefore the electric field $\vec{E}_{1}$ can be derived from the gradient of a scalar potential $\phi_{1}$ viz. $\vec{E}_{1}=-\nabla \phi_{1}$. Perturbations in the magnetic field are therefore neglected. The electrostatic modes occur due to charge separation and bunching phenomena. In our model all species are assumed to be magnetized.

The velocity distribution of the anisotropic ion beam is assumed to be a drifting Maxwellian with temperatures $T_{\perp}\left(T_{\|}\right)$perpendicular (parallel) to the external magnetic field. The beam is assumed to have drifts $V_{o y}\left(V_{o z}\right)$ perpendicular (parallel) to 
$\vec{B}_{\circ}$. The configuration is depicted below.

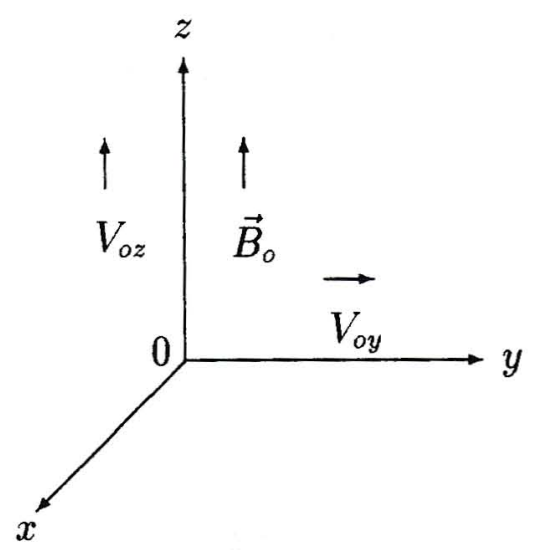

Figure 3.1

The equilibrium ion beam velocity distribution is given by

$$
\begin{gathered}
f_{i o}=\frac{n_{i o}}{\left(2 \pi C_{\perp}^{2}\right)\left(2 \pi C_{\|}^{2}\right)^{1 / 2}} \exp \left\{-\left[V_{x}^{2}+\left(V_{y}-V_{o y}\right)^{2}\right] / 2 C_{\perp}^{2}\right\} \times \\
\exp \left[-\left(V_{z}-V_{o z}\right)^{2} / 2 C_{\|}^{2}\right]
\end{gathered}
$$

where $C_{\perp}=\left(T_{\perp} / m_{i}\right)^{1 / 2}$ and $C_{\|}=\left(T_{\|} / m_{i}\right)^{1 / 2}$ are the perpendicular (parallel) thermal velocities of the beam ions respectively. The density of the ion beam is given by $n_{i o}$. In the linearization of the Vlasov equation

$$
\frac{\partial f_{i}}{\partial t}+\vec{V} \cdot \nabla f_{i}+\frac{\mathrm{d} \vec{V}}{\mathrm{~d} t} \cdot \frac{\partial f_{i}}{\partial \vec{V}}=0
$$

we consider small perturbations about equilibrium quantities: 


$$
\begin{aligned}
f_{i} & =f_{i o}+f_{i 1} \\
\vec{E} & =\vec{E}_{o}+\vec{E}_{1} \quad\left(\vec{E}_{o}=0\right) \\
\vec{B} & =\vec{B}_{o}+\vec{B}_{1} \\
n_{j} & =n_{j o}+n_{j 1}
\end{aligned}
$$

where $j=i, e$ and $d$ for the beam ions, electrons and dust particles respectively. Using the equations of motion for the ions

$$
\frac{\mathrm{d} \vec{V}}{\mathrm{~d} t}=\frac{e}{m_{i}}\left[\vec{E}_{o}+\vec{E}_{1}+\frac{\vec{V} \times \vec{B}_{o}}{c}+\frac{\vec{V} \times \vec{B}_{1}}{c}\right]
$$

the Vlasov equation for the beam now becomes

$$
\frac{\partial f_{i 1}}{\partial t}+\vec{V} \cdot \nabla f_{i 1}+\frac{e}{m_{i}}\left[\vec{E}_{o}+\frac{\vec{V} \times \vec{B}_{o}}{c}\right] \cdot \frac{\partial f_{i 1}}{\partial \vec{V}}=-\frac{e}{m_{i}}\left[\vec{E}_{1}+\frac{\vec{V} \times \vec{B}_{1}}{c}\right] \cdot \frac{\partial f_{i o}}{\partial \vec{V}}
$$

Since for electrostatic modes $\vec{E}_{1}=-\nabla \phi_{1}, \vec{B}_{1}=0$ and setting $\vec{E}_{o}=0$, the above equation can be rewritten as

$$
\frac{\mathrm{d} f_{i 1}}{\mathrm{~d} t}=\frac{e}{m_{i}} \nabla \phi_{1} \cdot \frac{\partial f_{i o}}{\partial \vec{V}}
$$

where the operator $d / d t$ is defined to be the rate of change following an unperturbed orbit in phase space (Gary et al.,1970). Integrating (3.6) along unperturbed orbits we obtain

$$
f_{i 1}(\vec{r}, \vec{V}, t)=\frac{e}{m_{i}} \int_{-\infty}^{t} \nabla \phi_{1}\left(\overrightarrow{r^{\prime}}, t^{\prime}\right) \cdot \frac{\partial f_{i o}\left(\vec{V}^{\prime}\right)}{\partial \vec{V}^{\prime}} \mathrm{d} t^{\prime}
$$

where $\quad \vec{V}^{\prime}=\frac{\mathrm{d} \vec{r}^{\prime}}{\mathrm{d} t^{\prime}}, \quad \frac{\mathrm{d} \vec{V}^{\prime}}{\mathrm{d} t^{\prime}}=\frac{e}{m_{i}}\left[\vec{E}_{o}+\frac{\vec{V}^{\prime} \times \vec{B}_{o}}{c}\right], \quad \overrightarrow{r^{\prime}}(0)=\vec{r}$ and $\quad \vec{V}^{\prime}(0)=\vec{V}$ 
Assuming the variation of perturbed quantities to be harmonic in space and time

$$
\begin{gathered}
f_{i 1}(\vec{r}, \vec{V}, t)=f_{i 1}(\vec{V}) \exp \{\mathrm{i}[\vec{k} \cdot \vec{r}-\omega t]\} \\
\phi_{1}(\vec{r}, t)=\phi_{1 k \omega} \exp \{\mathrm{i}[\vec{k} \cdot \vec{r}-\omega t]\}
\end{gathered}
$$

Then $\nabla \phi_{1}\left(\overrightarrow{r^{\prime}}, t^{\prime}\right)=i k \phi_{1}\left(\overrightarrow{r^{\prime}}, \mathrm{t}^{\prime}\right)$ and $(3.7)$ becomes

$$
f_{i 1}(\vec{r}, \vec{V}, t)=-\frac{\mathrm{i} e}{m_{i}} \int_{-\infty}^{t} \vec{k} \cdot \vec{V}_{e q} \phi_{1}\left(\vec{r}^{\prime}, t^{\prime}\right) f_{i o}\left(\vec{V}^{\prime}\right) \mathrm{d} t^{\prime}
$$

where

$$
\vec{V}_{e q}=-\left[\frac{V_{x}^{\prime}}{C_{\perp}^{2}} ; \frac{V_{y}^{\prime}-V_{o y}}{C_{\perp}^{2}} ; \frac{V_{z}^{\prime}-V_{o z}}{C_{\|}^{2}}\right]
$$

is obtained upon differentiation of (3.1).

Solving the equations of motion (3.4) for the ions we obtain

$$
\begin{aligned}
V_{x}^{\prime} & =V_{\perp}^{\prime} \cos \left(-\Omega_{i} t^{\prime}+\Theta\right) \\
V_{y}^{\prime}-V_{o y} & =V_{\perp}^{\prime} \sin \left(-\Omega_{i} t^{\prime}+\Theta\right) \\
V_{z}^{\prime} & =\text { constant }
\end{aligned}
$$

where $V_{\perp}^{\prime}=\left\{V_{x}^{\prime 2}+\left(V_{y}^{\prime}-V_{o y}\right)^{2}\right\}^{1 / 2}$ is the speed in a circular orbit in a plane perpendicular to the magnetic field. The configuration at $t^{\prime}=0$ is indicated in Figure 3.2.

Resolving $\vec{k}$ into components parallel and perpendicular to $\vec{B}_{o}$ (Figure 3.3 )

$$
\vec{k}=\left[k_{x}, k_{y}, k_{z}\right]=\left[k_{\perp} \cos \psi ; k_{\perp} \sin \psi ; k_{z}\right]
$$




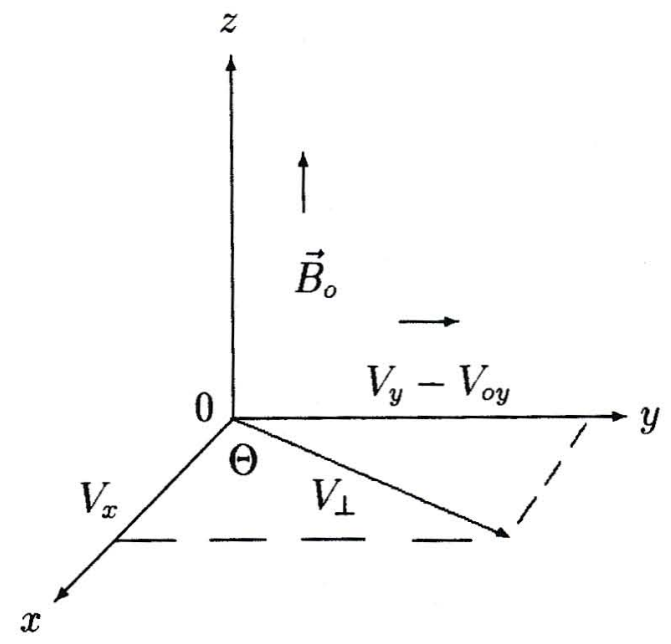

Figure 3.2

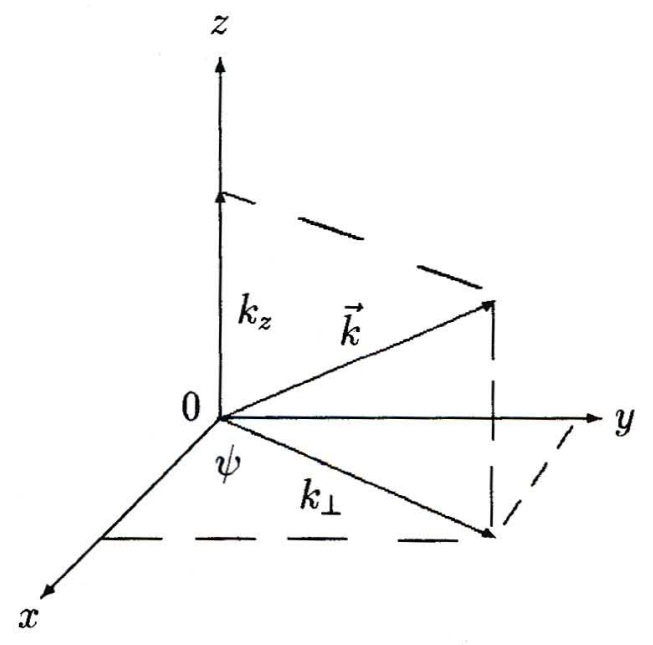

Figure 3.3 
We can show that

$$
\vec{k} \cdot \vec{V}_{e q}=\frac{k_{\perp} V_{\perp}^{\prime}}{C_{\perp}^{2}} \cos \left(\Omega_{i} t^{\prime}+\psi-\Theta\right)+\frac{k_{z}}{C_{\|}^{2}}\left(V_{z}^{\prime}-V_{o z}\right)
$$

Using (3.14) and (3.8), the integral (3.10) can be rewritten as

$$
\begin{aligned}
f_{i 1}(\vec{V}) \exp \{\mathrm{i}[\vec{k} \cdot \vec{r}-\omega t]\}= & f_{i 1}(\vec{r}, \vec{V}, t) \\
= & -\frac{\mathrm{i} e}{m_{i}} f_{i o}(\vec{V})\left[\frac{k_{\perp} V_{\perp}}{C_{\perp}^{2}} \int_{-\infty}^{t} \phi_{1} \cos \left(\Omega_{i} t^{\prime}+\psi-\Theta\right) \mathrm{d} t^{\prime}\right. \\
& \left.+\frac{k_{z}}{C_{\|}^{2}}\left(V_{z}^{\prime}-V_{o z}\right) \int_{-\infty}^{t} \phi_{1} \mathrm{~d} t^{\prime}\right]
\end{aligned}
$$

Evaluating the right hand side of $(3.15)$ at $t=0$ we get

$$
\begin{aligned}
f_{i 1}(\vec{V})= & -\frac{\mathrm{i} e}{m_{i}} f_{i o}(\vec{V}) \phi_{1 k \omega}\left[\frac{k_{\perp} V_{\perp}}{C_{\perp}^{2}} \int_{-\infty}^{0} \cos \left(\Omega_{i} t^{\prime}+\psi-\Theta\right) \exp \left\{\mathrm{i}\left[\vec{k} \cdot\left(\overrightarrow{r^{\prime}}-\vec{r}\right)-\omega t^{\prime}\right]\right\} d t^{\prime}\right. \\
& \left.+\frac{k_{z}}{C_{\|}^{2}}\left(V_{z}^{\prime}-V_{o z}\right) \int_{-\infty}^{0} \exp \left\{\mathrm{i}\left[\vec{k} \cdot\left(\overrightarrow{r^{\prime}}-\vec{r}\right)-\omega t^{\prime}\right]\right\} \mathrm{d} t^{\prime}\right]
\end{aligned}
$$

We solve the equations (3.12) with $\overrightarrow{r^{\prime}}(0)=\vec{r}=\left[x_{o}, y_{o}, z_{o}\right]$ to obtain the approximate orbit equations

$$
\begin{array}{r}
\overrightarrow{r^{\prime}}-\vec{r}=\frac{V_{\perp}^{\prime}}{\Omega_{i}}\left[\sin \Theta-\sin \left(-\Omega_{i} t^{\prime}+\Theta\right)\right] \hat{x} \\
+\left[\frac{V_{\perp}^{\prime}}{\Omega_{i}}\left\{-\cos \Theta+\cos \left(-\Omega_{i} t^{\prime}+\Theta\right)\right\}+V_{o y} t^{\prime}\right] \hat{y}+V_{z}^{\prime} t^{\prime} \hat{z}
\end{array}
$$

Using (3.13) and (3.17) the second integral in (3.16) reduces to

$$
\begin{array}{r}
\int_{-\infty}^{0} \exp [\mathrm{i} \mu \sin (\Theta-\psi)] \exp \left[-\mathrm{i} \mu \sin \left(\Theta-\psi-\Omega_{i} t^{\prime}\right)\right] \times \\
\exp \left[\mathrm{i}\left\{\vec{k} \cdot \vec{V}_{o}+k_{z}\left(V_{z}^{\prime}-V_{o z}\right)-\omega\right\} t^{\prime}\right] \mathrm{d} t^{\prime}
\end{array}
$$

where $\mu=k_{\perp} V_{\perp} / \Omega_{i}$. Using the identity (Watson,1944)

$$
\exp (\mathrm{i} \mu \sin \beta)=\sum_{l=-\infty}^{\infty} \exp (\mathrm{i} l \beta) J_{l}(\mu)
$$


where $J_{l}$ is the Bessel function of order $l$, the integral (3.18) becomes

$$
\sum_{\mathrm{p}=-\infty}^{\infty} \sum_{\mathrm{q}=-\infty}^{\infty} \frac{\exp [\mathrm{i}(\mathrm{p}-\mathrm{q})(\Theta-\psi)] J_{\mathrm{p}}(\mu) J_{\mathrm{q}}(\mu)}{\mathrm{i}\left[\mathrm{q} \Omega_{i}+\vec{k} \cdot \vec{V}_{o}+k_{z}\left(V_{z}-V_{o z}\right)-\omega\right]}
$$

The first integral in (3.16) can be manipulated by using the identity

$$
\cos \beta=\frac{e^{\mathrm{i} \beta}+e^{-\mathrm{i} \beta}}{2}
$$

and can be separated into two parts, of which the first part is given by

$$
\frac{1}{2} \int_{-\infty}^{0} \exp \left[\mathrm{i}\left(\psi-\Theta+\Omega_{i} t^{\prime}\right)\right] \exp \left[\mathrm{i}\left\{\vec{k} \cdot\left(\overrightarrow{r^{\prime}}-\vec{r}\right)-\omega t^{\prime}\right\}\right] \mathrm{d} t^{\prime}
$$

Using (3.17) and (3.19), the first part of the first integral of (3.16) simplifies to

$$
\frac{1}{2} \sum_{\mathrm{p}=-\infty}^{\infty} \sum_{\mathrm{q}=-\infty}^{\infty} \frac{\exp [\mathrm{i}(\mathrm{p}-\mathrm{q}-1)(\Theta-\psi)] J_{\mathrm{p}}(\mu) J_{\mathrm{q}}(\mu)}{\mathrm{i}\left[(\mathrm{q}+1) \Omega_{i}+\vec{k} \cdot \vec{V}_{o}+k_{z}\left(V_{z}-V_{o z}\right)-\omega\right]}
$$

Similarly the second part yields

$$
\frac{1}{2} \sum_{\mathrm{p}=-\infty}^{\infty} \sum_{\mathrm{q}=-\infty}^{\infty} \frac{\exp [\mathrm{i}(\mathrm{p}-\mathrm{q}+1)(\Theta-\psi)] J_{\mathrm{p}}(\mu) J_{\mathrm{q}}(\mu)}{\mathrm{i}\left[(\mathrm{q}-1) \Omega_{i}+\vec{k} \cdot \vec{V}_{o}+k_{z}\left(V_{z}-V_{o z}\right)-\omega\right]}
$$

Using (3.20)-(3.24) in (3.16) we obtain

$$
\begin{gathered}
f_{i 1}(\vec{V})=-\frac{e}{m_{i}} f_{i o}(\vec{V}) \phi_{1 k \omega}\left[\frac{k_{\perp} V_{\perp}}{2 C_{\perp}^{2}} \sum_{\mathrm{p}=-\infty}^{\infty} \sum_{\mathrm{q}=-\infty}^{\infty} J_{\mathrm{p}}(\mu) J_{\mathrm{q}}(\mu) \times\right. \\
\left\{\frac{\exp [\mathrm{i}(\mathrm{p}-\mathrm{q}-1)(\Theta-\psi)]}{\left[(\mathrm{q}+1) \Omega_{i}+\vec{k} \cdot \vec{V}_{o}+k_{z}\left(V_{z}-V_{o z}\right)-\omega\right]}\right. \\
\left.+\frac{\exp [\mathrm{i}(\mathrm{p}-\mathrm{q}+1)(\Theta-\psi)]}{\left[(\mathrm{q}-1) \Omega_{i}+\vec{k} \cdot \vec{V}_{o}+k_{z}\left(V_{z}-V_{o z}\right)-\omega\right]}\right\} \\
\left.+\frac{k_{z}}{C_{\|}^{2}}\left(V_{z}-V_{o z}\right) \sum_{\mathrm{p}=-\infty}^{\infty} \sum_{\mathrm{q}=-\infty}^{\infty} \frac{\exp [\mathrm{i}(\mathrm{p}-\mathrm{q})(\Theta-\psi)] J_{\mathrm{p}}(\mu) J_{\mathrm{q}}(\mu)}{\left[\mathrm{q} \Omega_{i}+\vec{k} \cdot \vec{V}_{o}+k_{z}\left(V_{z}-V_{o z}\right)-\omega\right]}\right]
\end{gathered}
$$

The perturbed ion beam density is given by 


$$
n_{i 1}=n_{i 1 k \omega} \exp \{\mathrm{i}[\vec{k} \cdot \vec{r}-\omega t]\}=\int f_{i 1}(\vec{V}) \mathrm{d}^{3} \vec{V}
$$

The integration can be manipulated by transforming to cylindrical co-ordinates in velocity space, with

$$
\mathrm{d}^{3} \vec{V}=V_{\perp} \mathrm{d} V_{\perp} \mathrm{d} V_{z} \mathrm{~d} \Theta
$$

The triple integral (3.26) can be separated into three parts. Using (3.1) for the equilibrium velocity distribution $f_{i o}(\vec{V})$, the first part is

$$
\begin{aligned}
-\frac{\pi e \phi_{1 k \omega} k_{\perp} n_{i o}}{T_{\perp}\left(2 \pi C_{\perp}^{2}\right)\left(2 \pi C_{\|}^{2}\right)^{1 / 2}} \sum_{\mathrm{p}=-\infty}^{\infty} \int_{0}^{\infty} & {\left[\int_{-\infty}^{\infty} \frac{\exp \left\{-\left[\left(V_{z}-V_{o z}\right)^{2} / 2 C_{\|}^{2}\right]\right\}}{\left[\mathrm{p} \Omega_{i}+\vec{k} \cdot \vec{V}_{o}+k_{z}\left(V_{z}-V_{o z}\right)-\omega\right]} \mathrm{d} V_{z}\right] \times } \\
& \exp \left(-\frac{V_{\perp}^{2}}{2 C_{\perp}^{2}}\right) J_{\mathrm{p}}(\mu) J_{\mathrm{p}-1}(\mu) V_{\perp}^{2} \mathrm{~d} V_{\perp}
\end{aligned}
$$

where we use

$$
\int_{0}^{2 \pi} \exp [\mathrm{i}(\mathrm{p}-\mathrm{q}-1) \Theta] \mathrm{d} \Theta= \begin{cases}0 & \text { if } \mathrm{p} \neq \mathrm{q}+1 \\ 2 \pi & \text { if } \mathrm{p}=\mathrm{q}+1\end{cases}
$$

Introducing the plasma dispersion function or the Z-function (Fried and Conte,1961)

$$
Z(\lambda)=\frac{1}{\sqrt{\pi}} \int_{-\infty}^{\infty} \frac{e^{-x^{2}}}{x-\lambda} \mathrm{d} x
$$

for $\operatorname{Im}(\lambda)>0$ or alternatively as

$$
Z(\lambda)=2 \mathrm{i} e^{-\lambda^{2}} \int_{-\infty}^{\mathrm{i} \lambda} e^{-t^{2}} \mathrm{~d} t
$$

the integral over $\mathrm{d} V_{z}$ in terms of the Z-function becomes

$$
\begin{aligned}
& -\frac{\pi e \phi_{1 k \omega} k_{\perp} n_{i o}}{T_{\perp}\left(2 \pi C_{\perp}^{2}\right)\left(2 \pi C_{\|}^{2}\right)^{1 / 2}} \frac{\sqrt{\pi}}{k_{z}} \sum_{\mathrm{p}=-\infty}^{\infty} \int_{0}^{\infty} J_{\mathrm{p}}(\mu) J_{\mathrm{p}-1}(\mu) Z\left(\frac{\omega-\vec{k} \cdot \vec{V}_{o}-\mathrm{p} \Omega_{i}}{\sqrt{2} k_{z} C_{\|}}\right) \times \\
& \exp \left(-\frac{V_{\perp}^{2}}{2 C_{\perp}^{2}}\right) V_{\perp}^{2} \mathrm{~d} V_{\perp}
\end{aligned}
$$


The second part of the integral (3.26) yields

$$
\begin{gathered}
-\frac{\pi e \phi_{1 k \omega} k_{\perp} n_{i o}}{T_{\perp}\left(2 \pi C_{\perp}^{2}\right)\left(2 \pi C_{\|}^{2}\right)^{1 / 2}} \frac{\sqrt{\pi}}{k_{z}} \sum_{\mathrm{p}=-\infty}^{\infty} \int_{0}^{\infty} J_{\mathrm{p}}(\mu) J_{\mathrm{p}+1}(\mu) Z\left(\frac{\omega-\vec{k} \cdot \vec{V}_{o}-\mathrm{p} \Omega_{i}}{\sqrt{2} k_{z} C_{\|}}\right) \times \\
\exp \left(-\frac{V_{\perp}^{2}}{2 C_{\perp}^{2}}\right) V_{\perp}^{2} \mathrm{~d} V_{\perp}
\end{gathered}
$$

It can be shown that the last part of the integral (3.26) is

$$
-\frac{e \phi_{1 k \omega} n_{i o}}{T_{\|} C_{\perp}^{2}} \sum_{\mathrm{p}=-\infty}^{\infty} \int_{0}^{\infty} J_{\mathrm{p}}^{2}(\mu)\left[1+z_{p i} Z\left(z_{p i}\right)\right] \exp \left(-\frac{V_{\perp}^{2}}{2 C_{\perp}^{2}}\right) V_{\perp} \mathrm{d} V_{\perp}
$$

where $z_{p i}=\left(\omega-\vec{k} \cdot \vec{V}_{o}-\mathrm{p} \Omega_{i}\right) / \sqrt{2} k_{z} C_{\|}$.

Combining (3.31), (3.32) and (3.33) we finally obtain

$$
\begin{array}{r}
n_{i 1 k \omega}=-\frac{e \phi_{1 k \omega} n_{i o}}{C_{\perp}^{2}}\left[\frac{1}{\sqrt{2} k_{z} C_{\|}} \sum_{\mathrm{p}=-\infty}^{\infty} \int_{0}^{\infty} \mathrm{p} \Omega_{i} J_{\mathrm{p}}{ }^{2}(\mu) Z\left(z_{p i}\right) \exp \left(-\frac{V_{\perp}^{2}}{2 C_{\perp}^{2}}\right) V_{\perp} \mathrm{d} V_{\perp}\right. \\
\left.+\frac{T_{\perp}}{T_{\|}} \sum_{\mathrm{p}=-\infty}^{\infty} \int_{0}^{\infty} J_{\mathrm{p}}{ }^{2}(\mu)\left[1+z_{p i} Z\left(z_{p i}\right)\right] \exp \left(-\frac{V_{\perp}^{2}}{2 C_{\perp}^{2}}\right) V_{\perp} \mathrm{d} V_{\perp}\right]
\end{array}
$$

where we have used the identity (Watson,1944)

$$
J_{\mathrm{p}-1}(\mu)+J_{\mathrm{p}+1}(\mu)=\frac{2 \mathrm{p} J_{\mathrm{p}}(\mu)}{\mu}
$$

where $\mu=k_{\perp} V_{\perp} / \Omega_{i}$.

Using the identity (Watson,1944) where $I_{\mathrm{p}}$ is the modified Bessel function of order $\mathrm{p}$

$$
\int_{0}^{\infty} J_{\mathrm{p}}^{2}(\beta x) \exp \left(-\alpha x^{2}\right) x \mathrm{~d} x=\frac{1}{2 \alpha} \exp \left(-\frac{\beta^{2}}{2 \alpha}\right) I_{\mathrm{p}}\left(\frac{\beta^{2}}{2 \alpha}\right),
$$


the expression for the perturbed density (3.34) becomes

$$
\begin{aligned}
n_{i 1 k \omega}=-\frac{e \phi_{1 k \omega} n_{i o}}{T_{\perp}} & {\left[\frac{1}{\sqrt{2} k_{z} C_{\|}} \sum_{\mathrm{p}=-\infty}^{\infty} \mathrm{p} \Omega_{i} Z\left(z_{p i}\right) \exp \left(-\frac{k_{\perp}^{2} C_{\perp}^{2}}{\Omega_{i}^{2}}\right) I_{\mathrm{p}}\left(\frac{k_{\perp}^{2} C_{\perp}^{2}}{\Omega_{i}^{2}}\right)\right.} \\
+ & \left.\frac{T_{\perp}}{T_{\|}} \sum_{\mathrm{p}=-\infty}^{\infty}\left[1+z_{p i} Z\left(z_{p i}\right)\right] \exp \left(-\frac{k_{\perp}^{2} C_{\perp}^{2}}{\Omega_{i}^{2}}\right) I_{\mathrm{p}}\left(\frac{k_{\perp}^{2} C_{\perp}^{2}}{\Omega_{i}^{2}}\right)\right]
\end{aligned}
$$

Finally letting $\alpha_{i}=k_{\perp}^{2} C_{\perp}^{2} / \Omega_{i}^{2}$ and $\Gamma_{p i}=e^{-\alpha_{i}} I_{\mathrm{p}}\left(\alpha_{i}\right)$, the equation (3.37) can be rewritten as

$$
\begin{aligned}
n_{i 1 k \omega}=-\frac{e \phi_{1 k \omega} n_{i o}}{T_{\perp}} & {\left[\frac{T_{\perp}}{T_{\|}}\left\{1+\frac{\omega-\vec{k} \cdot \vec{V}_{o}}{\sqrt{2} k_{z} C_{\|}} \sum_{\mathrm{p}=-\infty}^{\infty} Z\left(z_{p i}\right) \Gamma_{p i}\right\}\right.} \\
+ & \left.\left\{1-\frac{T_{\perp}}{T_{\|}}\right\} \sum_{\mathrm{p}=-\infty}^{\infty} \frac{\mathrm{p} \Omega_{i}}{\sqrt{2} k_{z} C_{\|}} Z\left(z_{p i}\right) \Gamma_{p i}\right]
\end{aligned}
$$

\subsection{Contribution of Background Species to Dis- persion Relation}

The stationary, isotropic Maxwellian distribution of the background electrons and dust particles is given by

$$
f_{j o}=\frac{n_{j o}}{\left(2 \pi C_{j}^{2}\right)^{3 / 2}} \exp \left(-\frac{V^{2}}{2 C_{j}^{2}}\right)
$$

where $j=e$ and $d$ for the electron and dust species.

Quasi-neutrality at equilibrium requires $n_{i o}=n_{e o}+Z_{d} n_{d o}$ where $e, d$ and $i$ refers to the background electron,dust grains and the beam ions respectively. Here $Z_{d}$ is the number of electronic charges on each dust grain. The perturbed densities for the background electrons and dust grains are easily obtained from (3.38) by setting 
$T_{\perp}=T_{\|}=T_{j}$ and $V_{o}=0$ for the isotropic, stationary species. We thus obtain

$$
n_{j 1 k \omega}=-\frac{q_{j} \phi_{1 k \omega} n_{j o}}{T_{j}}\left[1+\frac{\omega}{\sqrt{2} k_{z} C_{j}} \sum_{\mathrm{p}=-\infty}^{\infty} Z\left(\frac{\omega-\mathrm{p} \Omega_{j}}{\sqrt{2} k_{z} C_{j}}\right) \Gamma_{p j}\right]
$$

for $j=e$ and $d$ for the background electrons and dust particles respectively, with $q_{e}=-e$ and $q_{d}=-Z_{d} e$. In the electrostatic approximation, Poisson's equation

$$
\nabla^{2} \phi=4 \pi e\left[n_{e}+Z_{d} n_{d}-n_{i}\right]
$$

becomes

$$
\phi_{1 k \omega}=\frac{4 \pi e}{k^{2}}\left(n_{i 1 k \omega}-Z_{d} n_{d 1 k \omega}-n_{e 1 k \omega}\right)
$$

We finally obtain using (3.40) for the background dust particles and electrons and (3.38) for the beam ions

$$
\begin{aligned}
k^{2}+\frac{1}{\lambda_{d e}^{2}}\left[1+\frac{\omega}{\sqrt{2} k_{z} C_{e}} \sum_{\mathrm{p}=-\infty}^{\infty} Z\left(z_{p e}\right) \Gamma_{p e}\right] \\
+\frac{1}{\lambda_{d d}^{2}}\left[1+\frac{\omega}{\sqrt{2} k_{z} C_{d}} \sum_{\mathrm{p}=-\infty}^{\infty} Z\left(z_{p d}\right) \Gamma_{p d}\right] \\
+\frac{1}{\lambda_{d i}^{2}}\left[\sigma\left\{1+\frac{\omega-\vec{k} \cdot \vec{V}_{o}}{\sqrt{2} k_{z} C_{\|}} \sum_{\mathrm{p}=-\infty}^{\infty} Z\left(z_{p i}\right) \Gamma_{p i}\right\}\right. \\
\left.+\frac{\beta}{\sqrt{2} k_{z} C_{\|}} \sum_{\mathrm{p}=-\infty}^{\infty} \mathrm{p} \Omega_{i} Z\left(z_{p i}\right) \Gamma_{p i}\right]=0
\end{aligned}
$$

which is the dispersion relation for electrostatic waves in a dusty plasma comprising of an ion beam against a background of stationary electrons and dust grains, where all species are magnetized. Here $\sigma=T_{\perp} / T_{\|}, \beta=1-\sigma, z_{p e}=\left(\omega-\mathrm{p} \Omega_{e}\right) / \sqrt{2} k_{z} C_{e}$, $z_{p d}=\left(\omega-\mathrm{p} \Omega_{d}\right) / \sqrt{2} k_{z} C_{d}, z_{p i}=\left(\omega-\vec{k} \cdot \vec{V}_{o}-\mathrm{p} \Omega_{i}\right) / \sqrt{2} k_{z} C_{\|}$and the Debye length of species $j$ is defined by $\lambda_{d j}=\left(T_{j} / 4 \pi n_{j o} q_{j}^{2}\right)^{1 / 2}(j=e, i$ and $d)$. 


\subsection{Approximate Solutions of the Kinetic Disper- sion Relation}

From (3.43), the dispersion relation for a three-component dusty plasma (single dust component) (Bharuthram and Pather,1996) may be written as

$$
\begin{aligned}
0=k^{2} \lambda_{d}^{2} & +\frac{\lambda_{d}^{2}}{\lambda_{d e}^{2}}\left[1+\frac{\omega}{\sqrt{2} k_{z} C_{e}} \sum_{\mathrm{p}=-\infty}^{\infty} Z\left(z_{p e}\right) \Gamma_{p e}\right] \\
& +\frac{\lambda_{d}^{2}}{\lambda_{d i}^{2}}\left[1+\frac{\omega-\vec{k} \cdot \vec{V}_{o}}{\sqrt{2} k_{z} C_{i}} \sum_{\mathrm{p}=-\infty}^{\infty} Z\left(z_{p i}\right) \Gamma_{p i}\right] \\
& +\frac{\lambda_{d}^{2}}{\lambda_{d d}^{2}}\left[1+\frac{\omega}{\sqrt{2} k_{z} C_{d}} \sum_{\mathrm{p}=-\infty}^{\infty} Z\left(z_{p d}\right) \Gamma_{p d}\right]
\end{aligned}
$$

where $\lambda_{d}=\left(T_{e} / 4 \pi n_{i o} e^{2}\right)^{1 / 2}$. On the other hand, in the presence of two dust species of mass $m_{d l}, m_{d h}$ and charge $-Z_{d l} e,-Z_{d h} e$; the general dispersion relation will be

$$
\begin{aligned}
0=k^{2} \lambda_{d}^{2} & +\frac{\lambda_{d}^{2}}{\lambda_{d e}^{2}}\left[1+\frac{\omega}{\sqrt{2} k_{z} C_{e}} \sum_{\mathrm{p}=-\infty}^{\infty} Z\left(z_{p e}\right) \Gamma_{p e}\right] \\
& +\frac{\lambda_{d}^{2}}{\lambda_{d i}^{2}}\left[1+\frac{\omega-\vec{k} \cdot \overrightarrow{V_{o}}}{\sqrt{2} k_{z} C_{i}} \sum_{\mathrm{p}=-\infty}^{\infty} Z\left(z_{p i}\right) \Gamma_{p i}\right] \\
& +\frac{\lambda_{d}^{2}}{\lambda_{d d l}^{2}}\left[1+\frac{\omega}{\sqrt{2} k_{z} C_{d l}} \sum_{\mathrm{p}=-\infty}^{\infty} Z\left(z_{p d l}\right) \Gamma_{p d l}\right] \\
& +\frac{\lambda_{d}^{2}}{\lambda_{d d h}^{2}}\left[1+\frac{\omega}{\sqrt{2} k_{z} C_{d h}} \sum_{\mathrm{p}=-\infty}^{\infty} Z\left(z_{p d h}\right) \Gamma_{p d h}\right]
\end{aligned}
$$

We consider low frequency waves in three and four-component plasmas with hot electrons, hot ions and cold dust constituents. Hence we make the assumptions $T_{e} \approx T_{i} \gg T_{d}\left(T_{e} \approx T_{i} \gg T_{d h} ; T_{d l}\right)$ for a three-component (four-component) plasma and $|\omega| \ll \Omega_{i}$. 
The series expansion of the Z-function (Fried and Conte,1961) is given by

$$
\begin{aligned}
& Z(z)=\mathrm{i} \sqrt{\pi} \delta e^{-z^{2}}-2 z\left[1-\frac{2 z^{2}}{3}+\frac{4 z^{4}}{15}-\ldots\right] \text { for }|z| \ll 1 \text { and } \\
& Z(z)=\mathrm{i} \sqrt{\pi} \delta e^{-z^{2}}-\frac{1}{z}\left[1+\frac{1}{2 z^{2}}+\frac{3}{4 z^{4}}+\ldots\right] \text { for }|z| \gg 1
\end{aligned}
$$

where

$$
\delta= \begin{cases}0 & \text { if } \operatorname{Im}(z)>0 \\ 1 & \text { if } \operatorname{Im}(z)=0 \\ 2 & \text { if } \operatorname{Im}(z)<0\end{cases}
$$

Assuming the drift of the ions to be weak (small $V_{o}$ ) (Rosenberg,1993), $|\bar{\omega}| \ll \Omega_{i}$ where $\bar{\omega}=\omega-\vec{k} \cdot \vec{V}_{o}$ is a Doppler shifted frequency. With this assumption

$$
z_{p i}=\frac{\omega-\vec{k} \cdot \vec{V}_{o}-\mathrm{p} \Omega_{i}}{\sqrt{2} k_{z} C_{i}} \approx \frac{-\mathrm{p} \Omega_{i}}{\sqrt{2} k_{z} C_{i}} \text { for } \mathrm{p} \neq 0 .
$$

Then

$$
\begin{aligned}
\sum_{\mathrm{p}=-\infty}^{\infty} Z\left(z_{p i}\right) \Gamma_{p i} \approx Z\left(\frac{\omega-\vec{k} \cdot \vec{V}_{o}}{\sqrt{2} k_{z} C_{i}}\right) \Gamma_{o i} & +\sum_{\mathrm{p}=1}^{\infty}\left\{Z\left(\frac{\mathrm{p} \Omega_{i}}{\sqrt{2} k_{z} C_{i}}\right)+Z\left(-\frac{\mathrm{p} \Omega_{i}}{\sqrt{2} k_{z} C_{i}}\right)\right\} \Gamma_{p i} \\
& =Z\left(z_{o i}\right) \Gamma_{o i}
\end{aligned}
$$

for $z_{o i}=\left(\omega-\vec{k} \cdot \vec{V}_{o}\right) / \sqrt{2} k_{z} C_{i}$ and using the fact that $Z(\xi)+Z(-\xi)=0$ which can easily be proved from the definition of the Z-function.

Furthermore for large $T_{i}$ we may assume that $\left|z_{o i}\right| \ll 1$. Then using (3.46) we may write

$$
\sum_{\mathrm{p}=-\infty}^{\infty} Z\left(z_{p i}\right) \Gamma_{p i} \approx\left(\mathrm{i} \sqrt{\pi}-2 z_{o i}\right) \Gamma_{o i}
$$

Since for the cold dust grains $\left|\alpha_{d}\right|=\left|k_{\perp}^{2} C_{d}^{2} / \Omega_{d}^{2}\right| \ll 1$ and $\Gamma_{\mathrm{p}}(x) \ll 1$ for $\mathrm{p} \neq 0$, we can write $\Gamma_{\mathrm{p}}(x)=e^{-x} I_{\mathrm{p}}(x) \approx(x / 2)^{\mathrm{p}}(1 / \mathrm{p} !)(1-x)$ for $|x| \ll 1$. Hence we retain only 
the zero order term in the summation. We may then write

$$
\sum_{\mathrm{p}=-\infty}^{\infty} Z\left(z_{p d}\right) \Gamma_{p d} \approx Z\left(z_{o d}\right) \Gamma_{o d} \approx\left[-\frac{1}{z_{o d}}-\frac{1}{2 z_{o d} 3}\right] \Gamma_{o d}
$$

where $z_{o d}=\omega / \sqrt{2} k_{z} C_{d}$.

Since $|\omega| \ll \Omega_{e}$,

$$
z_{p e}=\frac{\omega-\mathrm{p} \Omega_{e}}{\sqrt{2} k_{z} C_{e}} \approx \frac{-\mathrm{p} \Omega_{e}}{\sqrt{2} k_{z} C_{e}} \text { for } \mathrm{p} \neq 0
$$

Then

$$
\begin{aligned}
\sum_{\mathrm{p}=-\infty}^{\infty} Z\left(z_{p e}\right) \Gamma_{p e} \approx Z\left(\frac{\omega}{\sqrt{2} k_{z} C_{e}}\right) \Gamma_{o e} & +\sum_{\mathrm{p}=1}^{\infty}\left\{Z\left(\frac{\mathrm{p} \Omega_{e}}{\sqrt{2} k_{z} C_{e}}\right)+Z\left(-\frac{\mathrm{p} \Omega_{e}}{\sqrt{2} k_{z} C_{e}}\right)\right\} \Gamma_{p e} \\
& =Z\left(z_{o e}\right) \Gamma_{o e}
\end{aligned}
$$

for $z_{o e}=\omega / \sqrt{2} k_{z} C_{e}$ and again using $Z(\xi)+Z(-\xi)=0$.

For large $T_{e}$ we may assume that $\left|z_{o e}\right| \ll 1$. We may then write

$$
\sum_{\mathrm{p}=-\infty}^{\infty} Z\left(z_{p e}\right) \Gamma_{p e} \approx\left(\mathrm{i} \sqrt{\pi}-2 z_{o e}\right) \Gamma_{o e}
$$

Using (3.50),(3.51) and (3.54) in the dispersion relation (3.44) we obtain

$$
\begin{aligned}
0=k^{2} \lambda_{d}^{2}+ & \frac{n_{e o}}{n_{i o}}\left[1+z_{o e}\left(\mathrm{i} \sqrt{\pi}-2 z_{o e}\right) \Gamma_{o e}\right] \\
+ & \frac{T_{e}}{T_{i}}\left[1+z_{o i}\left(\mathrm{i} \sqrt{\pi}-2 z_{o i}\right) \Gamma_{o i}\right] \\
& +\frac{T_{e}}{T_{d}} \frac{n_{d o}}{n_{i o}} Z_{d}^{2}\left[1+z_{o d}\left(-\frac{1}{z_{o d}}-\frac{1}{2 z_{o d}}\right) \Gamma_{o d}\right]
\end{aligned}
$$

It can be shown that for $\alpha_{d}=k_{\perp}^{2} C_{d}^{2} / \Omega_{d}^{2} \ll 1$

$$
\Gamma_{o d} \approx 1
$$


Using (3.56) and neglecting terms involving $z_{o e}{ }^{2}$ and $z_{o i}{ }^{2}$ since $\left|z_{o e}\right|,\left|z_{o i}\right| \ll 1$; equation (3.55) becomes

$$
0=k^{2} \lambda_{d}^{2}+\frac{n_{e o}}{n_{i o}}+\frac{T_{e}}{T_{i}}+\frac{T_{e}}{T_{d}} \frac{n_{d o}}{n_{i o}} Z_{d}^{2}\left[-\frac{1}{2 z_{o d}^{2}}\right]
$$

Setting $\omega=\omega_{r}+\mathrm{i} \gamma$ and assuming $\left|\gamma / \omega_{r}\right| \ll 1$ we can write

$$
\begin{aligned}
\frac{1}{\omega^{2}}=\frac{1}{\omega_{r}^{2}+2 \mathrm{i} \gamma \omega_{r}-\gamma^{2}} & =\frac{1}{\omega_{r}^{2}\left(1+\frac{2 \mathrm{i} \gamma}{\omega_{r}}-\frac{\gamma^{2}}{\omega_{r}^{2}}\right)} \\
& =\frac{1}{\omega_{r}^{2}\left(1+\frac{\mathrm{i} \gamma}{\omega_{r}}\right)^{2}} \\
& =\frac{1}{\omega_{r}^{2}}\left[1-\frac{2 \mathrm{i} \gamma}{\omega_{r}}\right]
\end{aligned}
$$

Using the above manipulation, (3.57) becomes

$$
0=k^{2} \lambda_{d}^{2}+\frac{n_{e o}}{n_{\text {io }}}+\frac{T_{e}}{T_{i}}+\frac{T_{e}}{T_{d}} \frac{n_{d o}}{n_{i o}} Z_{d}^{2}\left(-\frac{k_{z}^{2} C_{d}^{2}}{\omega_{r}^{2}}\left[1-\frac{2 \mathrm{i} \gamma}{\omega_{r}}\right]\right)
$$

Taking the real part of the equation (3.59) we get

$$
0=k^{2} \lambda_{d}^{2}+\frac{n_{e o}}{n_{i o}}+\frac{T_{e}}{T_{i}}+\frac{T_{e}}{T_{d}} \frac{n_{d o}}{n_{i o}} Z_{d}^{2}\left(-\frac{k_{z}^{2} C_{d}^{2}}{\omega_{r}^{2}}\right)
$$

Multiplying (3.60) by $n_{i o} / n_{e o}$ and rearranging terms we obtain

$$
\frac{n_{i o}}{n_{e o}} k^{2} \lambda_{d}^{2}+1+\frac{n_{i o}}{n_{e o}} \frac{T_{e}}{T_{i}}=\frac{T_{e}}{T_{d}} \frac{T_{d}}{m_{d}} \frac{n_{i o}}{n_{e o}} \frac{n_{d o}}{n_{i o}} Z_{d}^{2}\left(\frac{k_{z}^{2}}{\omega_{r}^{2}}\right)
$$

We recall that the quasi-neutrality condition for a three-component dusty plasma is given by

$$
n_{e o}+Z_{d} n_{d o}=n_{i o}
$$


Using (3.62), the equation (3.61) becomes

$$
\frac{n_{i o}}{n_{e o}} k^{2} \lambda_{d}^{2}+1+\frac{n_{i o}}{n_{e o}} \frac{T_{e}}{T_{i}}=\frac{T_{e}}{T_{d}} \frac{n_{i o}}{n_{e o}} \frac{n_{i o}-n_{e o}}{Z_{d}} \frac{1}{n_{i o}} Z_{d}^{2} \frac{T_{d}}{m_{d}}\left(\frac{k_{z}^{2}}{\omega_{r}^{2}}\right)
$$

We finally obtain

$$
\omega_{r}^{2}=k_{z}^{2} C_{s}^{2} Z_{d}(\delta-1) \frac{m_{i}}{m_{d}} \frac{1}{\left(1+\delta \frac{T_{e}}{T_{i}}+\delta k^{2} \lambda_{d}^{2}\right)}
$$

where $C_{s}=\sqrt{T_{e} / m_{i}}$ and $\delta=n_{i o} / n_{e o}$. This expression corresponds to that obtained by Bharuthram and Pather (1996) for a three-component (single dust constituent) plasma.

Now for a four-component plasma (two dust species), the approximate dispersion relation from (3.45) is given by

$$
\begin{aligned}
0=k^{2} \lambda_{d}^{2} & +\frac{n_{e o}}{n_{i o}}\left[1+z_{o e}\left(\mathrm{i} \sqrt{\pi}-2 z_{o e}\right) \Gamma_{o e}\right] \\
+ & \frac{T_{e}}{T_{i}}\left[1+z_{o i}\left(\mathrm{i} \sqrt{\pi}-2 z_{o i}\right) \Gamma_{o i}\right] \\
+ & \frac{T_{e}}{T_{d h}} \frac{n_{d h o}}{n_{i o}} Z_{d h}^{2}\left[1+z_{o d h}\left(-\frac{1}{z_{o d h}}-\frac{1}{2 z_{o d h}{ }^{3}}\right) \Gamma_{o d h}\right] \\
& +\frac{T_{e}}{T_{d l}} \frac{n_{d l o}}{n_{i o}} Z_{d l}^{2}\left[1+z_{o d l}\left(-\frac{1}{z_{o d l}}-\frac{1}{2 z_{o d l}{ }^{3}}\right) \Gamma_{o d l}\right]
\end{aligned}
$$

where subscripts $d h$ and $d l$ have been introduced to differentiate between the heavy and the light dust components.

Simplifications are the same as before and the four-component analogue of equation (3.60) is given by

$$
0=k^{2} \lambda_{d}^{2}+\frac{n_{e o}}{n_{i o}}+\frac{T_{e}}{T_{i}}+\frac{T_{e}}{T_{d l}} \frac{n_{d l o}}{n_{i o}} Z_{d l}^{2}\left(-\frac{k_{z}^{2} C_{d l}^{2}}{\omega_{r}^{2}}\right)+\frac{T_{e}}{T_{d h}} \frac{n_{d h o}}{n_{i o}} Z_{d h}^{2}\left(-\frac{k_{z}^{2} C_{d h}^{2}}{\omega_{r}^{2}}\right)
$$


Multiplying (3.66) by $n_{i o} / n_{e o}$ and rearranging terms we get

$$
\frac{n_{i o}}{n_{e o}} k^{2} \lambda_{d}^{2}+1+\frac{n_{i o}}{n_{e o}} \frac{T_{e}}{T_{i}}=\left\{\frac{T_{e}}{T_{d l}} \frac{n_{i o}}{n_{e o}} \frac{n_{d l o}}{n_{i o}} Z_{d l}^{2} \frac{T_{d l}}{m_{d l}}+\frac{T_{e}}{T_{d h}} \frac{n_{i o}}{n_{e o}} \frac{n_{d h o}}{n_{i o}} Z_{d h}^{2} \frac{T_{d h}}{m_{d h}}\right\}\left(\frac{k_{z}^{2}}{\omega_{r}^{2}}\right)
$$

Using the quasi-neutrality condition

$$
n_{e o}+Z_{d l} n_{d l o}+Z_{d h} n_{d h o}=n_{i o}
$$

the expression within curly brackets becomes

$$
\left\{\frac{T_{e}}{m_{i}} \frac{m_{i}}{m_{d l}} Z_{d l}\left(\delta-1-Z_{d h} \frac{n_{d h o}}{n_{e o}}\right)+\frac{T_{e}}{m_{i}} \frac{m_{i}}{m_{d h}} Z_{d h}\left(\delta-1-Z_{d l} \frac{n_{d l o}}{n_{e o}}\right)\right\}
$$

We finally obtain

$$
\omega_{r}^{2}=k_{z}^{2} C_{s}^{2} \frac{\left\{\frac{m_{i}}{m_{d h}} Z_{d h}\left(\delta-1-Z_{d l} \frac{n_{d l o}}{n_{e o}}\right)+\frac{m_{i}}{m_{d l}} Z_{d l}\left(\delta-1-Z_{d h} \frac{n_{d h e}}{n_{e o}}\right)\right\}}{\left(1+\delta \frac{T_{e}}{T_{i}}+\delta k^{2} \lambda_{d}^{2}\right)}
$$

which is the approximate expression of the real frequency for a four-component plasma (two dust constituents).

Setting the density of either one of the dust constituents to be zero ( $n_{d l o}=0$ say), we can write from the quasi-neutrality condition (3.68)

$$
\frac{Z_{d h} n_{d h o}}{n_{e o}}=\delta-1
$$

Using (3.71), the approximate solution (3.70) becomes

$$
\omega_{r}^{2}=k_{z}^{2} C_{s}^{2} \frac{m_{i}}{m_{d h}} Z_{d h}(\delta-1) \frac{1}{\left(1+\delta \frac{T_{e}}{T_{i}}+\delta k^{2} \lambda_{d}^{2}\right)}
$$

which is the approximate solution of the full dispersion relation for the three-component (single dust constituent) plasma of Bharuthram and Pather (1996). 
In deriving the approximate solution of the growth rate for a three-component plasma, we neglect second order terms in (3.55) and we include the exponential term in the series expansion of the Z-function for the dust species.

$$
\begin{aligned}
0= & k^{2} \lambda_{d}^{2}+\frac{n_{e o}}{n_{i o}}\left[1+\mathrm{i} \sqrt{\pi} z_{o e} \Gamma_{o e}\right]+\frac{T_{e}}{T_{i}}\left[1+\mathrm{i} \sqrt{\pi} z_{o i} \Gamma_{o i}\right] \\
& +\frac{T_{e}}{T_{d}} \frac{n_{d o}}{n_{i o}} Z_{d}^{2}\left[-\frac{k_{z}^{2} C_{d}^{2}}{\omega^{2}}+\mathrm{i} \sqrt{\pi} z_{o d} e^{-z_{o d}{ }^{2}}\right]
\end{aligned}
$$

Now

$$
\begin{aligned}
z_{o d}^{2}=\left(\frac{\omega}{\sqrt{2} k_{z} C_{d}}\right)^{2} & =\frac{\omega_{r}^{2}\left(1+\frac{2 i \gamma}{\omega_{r}}-\frac{\gamma^{2}}{\omega_{r}^{2}}\right)}{2 k_{z}^{2} \frac{T_{d}}{m_{d}}} \\
& \approx \frac{\left(k_{z}^{2} \frac{T_{e}}{m_{i}} Z_{d}(\delta-1) \frac{m_{i}}{m_{d}} \frac{1}{\left(1+\delta \frac{T_{e}}{T_{i}}+\delta k^{2} \lambda_{d}^{2}\right)}\right)}{2 k_{z}^{2} \frac{T_{d}}{m_{d}}} \\
& =\frac{T_{e}}{2 T_{d}} Z_{d}(\delta-1) \frac{1}{\left(1+\delta \frac{T_{e}}{T_{i}}+\delta k^{2} \lambda_{d}^{2}\right)}
\end{aligned}
$$

using the expression (3.72) for $\omega_{r}$ and neglecting terms involving $\gamma / \omega_{r}$ assuming that $\left|\gamma / \omega_{r}\right| \ll 1$

Now using $z_{o e}=\omega / \sqrt{2} k_{z} C_{e}, z_{o i}=\left(\omega-\vec{k} \cdot \vec{V}_{o}\right) / \sqrt{2} k_{z} C_{i}$ and $z_{o d}=\omega / \sqrt{2} k_{z} C_{d}$ and writing $\omega=\omega_{r}+\mathrm{i} \gamma$; the equation (3.73) becomes

$$
\begin{aligned}
0=k^{2} \lambda_{d}^{2}+\frac{n_{e o}}{n_{i o}}\left[1+\mathrm{i} \sqrt{\pi} \frac{\omega_{r}+\mathrm{i} \gamma}{\sqrt{2} k_{z} C_{e}} \Gamma_{o e}\right] \\
+\frac{T_{e}}{T_{i}}\left[1+\mathrm{i} \sqrt{\pi} \frac{\omega_{r}+\mathrm{i} \gamma-\vec{k} \cdot \vec{V}_{o}}{\sqrt{2} k_{z} C_{i}} \Gamma_{o i}\right] \\
+\frac{T_{e}}{T_{d}} \frac{n_{d o}}{n_{i o}} Z_{d}^{2}\left[-\frac{k_{z}^{2} C_{d}^{2}}{\omega_{r}^{2}}\left(1-\frac{2 \mathrm{i} \gamma}{\omega_{r}}\right)+\mathrm{i} \sqrt{\pi} \frac{\omega_{r}+\mathrm{i} \gamma}{\sqrt{2} k_{z} C_{d}} e^{-z_{o d}{ }^{2}}\right]
\end{aligned}
$$


Taking the imaginary part of (3.75)

$$
\begin{gathered}
0=\frac{n_{e o}}{n_{i o}}\left(\frac{\pi}{2}\right)^{1 / 2} \frac{\omega_{r}}{k_{z}}\left(\frac{m_{e}}{T_{e}}\right)^{1 / 2} \Gamma_{o e}+\frac{T_{e}}{T_{i}}\left(\frac{\pi}{2}\right)^{1 / 2} \frac{\omega_{r}}{k_{z}}\left(\frac{m_{i}}{T_{i}}\right)^{1 / 2} \Gamma_{o i} \\
-\frac{T_{e}}{T_{i}}\left(\frac{\pi}{2}\right)^{1 / 2} \frac{\vec{k} \cdot \vec{V}_{o}}{k_{z}}\left(\frac{m_{i}}{T_{i}}\right)^{1 / 2} \Gamma_{o i}+\frac{T_{e}}{T_{d}} \frac{n_{d o}}{n_{i o}} Z_{d}^{2} \frac{2 \gamma}{\omega_{r}} \frac{k_{z}^{2}}{\omega_{r}^{2}} \frac{T_{d}}{m_{d}} \\
+\frac{T_{e}}{T_{d}} \frac{n_{d o}}{n_{i o}} Z_{d}^{2}\left(\frac{\pi}{2}\right)^{1 / 2} \frac{\omega_{r}}{k_{z}}\left(\frac{m_{d}}{T_{d}}\right)^{1 / 2} e^{-z_{o d}{ }^{2}}
\end{gathered}
$$

From the quasi-neutrality condition (3.62) we can write

$$
\frac{n_{d o}}{n_{\text {io }}}=\frac{1}{Z_{d}}\left(1-\frac{1}{\delta}\right)
$$

Using (3.77), the equation (3.76) can be written as

$$
\begin{gathered}
0=\frac{1}{\delta}\left(\frac{\pi}{2}\right)^{1 / 2} \frac{\omega_{r}}{k_{z}}\left(\frac{m_{e}}{T_{e}}\right)^{1 / 2} \Gamma_{o e}+\frac{T_{e}}{T_{i}}\left(\frac{\pi}{2}\right)^{1 / 2} \frac{\omega_{r}}{k_{z}}\left(\frac{m_{i}}{T_{i}}\right)^{1 / 2} \Gamma_{o i} \\
-\frac{T_{e}}{T_{i}}\left(\frac{\pi}{2}\right)^{1 / 2} \frac{\vec{k} \cdot \vec{V}_{o}}{k_{z}}\left(\frac{m_{i}}{T_{i}}\right)^{1 / 2} \Gamma_{o i}+\frac{T_{e}}{T_{d}} \frac{1}{Z_{d}}\left(1-\frac{1}{\delta}\right) Z_{d}^{2} \frac{2 \gamma}{\omega_{r}} \frac{k_{z}^{2}}{\omega_{r}^{2}} \frac{T_{d}}{m_{d}} \\
+\frac{T_{e}}{T_{d}} \frac{1}{Z_{d}}\left(1-\frac{1}{\delta}\right) Z_{d}^{2}\left(\frac{\pi}{2}\right)^{1 / 2} \frac{\omega_{r}}{k_{z}}\left(\frac{m_{d}}{T_{d}}\right)^{1 / 2} e^{-z_{o d}{ }^{2}}
\end{gathered}
$$

Simplifying equation (3.78) we get

$$
\begin{gathered}
0=\left(\frac{m_{e}}{m_{d}}\right)^{1 / 2} \Gamma_{o e}+\delta\left(\frac{T_{e}}{T_{i}}\right)^{3 / 2}\left(\frac{m_{i}}{m_{d}}\right)^{1 / 2} \Gamma_{o i}-\delta\left(\frac{T_{e}}{T_{i}}\right)^{3 / 2}\left(\frac{m_{i}}{m_{d}}\right)^{1 / 2} \frac{\vec{k} \cdot \vec{V}_{o}}{\omega_{r}} \Gamma_{o i} \\
+\left(\frac{T_{e}}{m_{d}}\right)^{3 / 2} \frac{2 \sqrt{2}}{\sqrt{\pi}} Z_{d}(\delta-1) \frac{k_{z}^{3}}{\omega_{r}^{3}} \frac{\gamma}{\omega_{r}}+\left(\frac{T_{e}}{T_{d}}\right)^{3 / 2} Z_{d}(\delta-1) e^{-z_{o d}{ }^{2}}
\end{gathered}
$$

Rearranging terms and making $\gamma$ the subject of (3.79)

$$
\begin{aligned}
\gamma=\left\{\left(\frac{m_{d}}{T_{e}}\right)^{3 / 2}\right. & \left.\frac{\sqrt{\pi}}{2 \sqrt{2}} \frac{1}{Z_{d}(\delta-1)} \frac{\omega_{r}^{4}}{k_{z}^{3}}\right\}\left[-\left(\frac{m_{e}}{m_{d}}\right)^{1 / 2} \Gamma_{o e}-\delta\left(\frac{T_{e}}{T_{i}}\right)^{3 / 2}\left(\frac{m_{i}}{m_{d}}\right)^{1 / 2} \Gamma_{o i}\right. \\
& \left.+\delta\left(\frac{T_{e}}{T_{i}}\right)^{3 / 2}\left(\frac{m_{i}}{m_{d}}\right)^{1 / 2} \frac{\vec{k} \cdot \vec{V}_{o}}{\omega_{r}} \Gamma_{o i}-\left(\frac{T_{e}}{T_{d}}\right)^{3 / 2} Z_{d}(\delta-1) e^{-z_{o d}{ }^{2}}\right]
\end{aligned}
$$


Now $\Gamma_{o i}\left(\alpha_{i}\right) \approx 1$ since $\alpha_{i} \ll 1$. Rearranging terms and using expression (3.64) for $\omega_{r}$ we finally get

$$
\begin{aligned}
\gamma=\left\{\sqrt{\frac{\pi}{8}}\right. & \left.\sqrt{Z_{d}(\delta-1)} \frac{\omega_{r}}{\left(1+\delta \frac{T_{e}}{T_{i}}+\delta k^{2} \lambda_{d}^{2}\right)^{3 / 2}}\right\}\left[-\left(\frac{m_{e}}{m_{d}}\right)^{1 / 2} \Gamma_{o e}\right. \\
+ & \delta\left(\frac{T_{e}}{T_{i}}\right)^{3 / 2}\left(\frac{m_{i}}{m_{d}}\right)^{1 / 2}\left(\frac{\vec{k} \cdot \vec{V}_{o}}{\omega_{r}}-1\right) \\
& \left.-\left(\frac{T_{e}}{T_{d}}\right)^{3 / 2} Z_{d}(\delta-1) \exp \left\{-\frac{T_{e}}{2 T_{d}} Z_{d}(\delta-1) \frac{1}{\left(1+\delta \frac{T_{e}}{T_{i}}+\delta k^{2} \lambda_{d}^{2}\right)}\right\}\right]
\end{aligned}
$$

which is the approximate expression of the growth rate derived by Bharuthram et al. (1996) for a three-component (single dust constituent) plasma. We note in (3.81) that the electrons and dust particles provide Landau damping, while for $\vec{k} \cdot \vec{V}_{o}>\omega_{r}$, the ions provide inverse Landau damping which leads to wave growth when the positive ion term dominates.

Extending the calculation to a plasma with two dust species, the four-component analogue of equation (3.75) is given by

$$
\begin{aligned}
0=k^{2} \lambda_{d}^{2}+ & \frac{n_{e o}}{n_{i o}}\left[1+\mathrm{i} \sqrt{\pi} \frac{\omega_{r}+\mathrm{i} \gamma}{\sqrt{2} k_{z} C_{e}} \Gamma_{o e}\right]+\frac{T_{e}}{T_{i}}\left[1+\mathrm{i} \sqrt{\pi} \frac{\omega_{r}+\mathrm{i} \gamma-\vec{k} \cdot \vec{V}_{o}}{\sqrt{2} k_{z} C_{i}} \Gamma_{o i}\right] \\
+ & \frac{T_{e}}{T_{d h}} \frac{n_{d h o}}{n_{i o}} Z_{d h}^{2}\left[-\frac{k_{z}^{2} C_{d h}^{2}}{\omega_{r}^{2}}\left(1-\frac{2 \mathrm{i} \gamma}{\omega_{r}}\right)+\mathrm{i} \sqrt{\pi} \frac{\omega_{r}+\mathrm{i} \gamma}{\sqrt{2} k_{z} C_{d h}} e^{-z_{o d h}{ }^{2}}\right] \\
& +\frac{T_{e}}{T_{d l}} \frac{n_{d l o}}{n_{i o}} Z_{d l}^{2}\left[-\frac{k_{z}^{2} C_{d l}^{2}}{\omega_{r}^{2}}\left(1-\frac{2 \mathrm{i} \gamma}{\omega_{r}}\right)+\mathrm{i} \sqrt{\pi} \frac{\omega_{r}+\mathrm{i} \gamma}{\sqrt{2} k_{z} C_{d l}} e^{-z_{o d l}{ }^{2}}\right]
\end{aligned}
$$


Taking the imaginary part of (3.82) and using the quasi-neutrality condition (3.68), the equation (3.82) becomes

$$
\begin{gathered}
0=\frac{1}{\delta}\left(\frac{\pi}{2}\right)^{1 / 2} \frac{\omega_{r}}{k_{z}}\left(\frac{m_{e}}{T_{e}}\right)^{1 / 2} \Gamma_{o e}+\frac{T_{e}}{T_{i}}\left(\frac{\pi}{2}\right)^{1 / 2} \frac{\omega_{r}}{k_{z}}\left(\frac{m_{i}}{T_{i}}\right)^{1 / 2} \Gamma_{o i}-\frac{T_{e}}{T_{i}}\left(\frac{\pi}{2}\right)^{1 / 2} \frac{\vec{k} \cdot \vec{V}_{o}}{k_{z}}\left(\frac{m_{i}}{T_{i}}\right)^{1 / 2} \Gamma_{o i} \\
+\frac{T_{e}}{T_{d h}} Z_{d h}\left(1-\frac{1}{\delta}-Z_{d l} \frac{n_{d l o}}{n_{i o}}\right) \frac{2 \gamma}{\omega_{r}} \frac{k_{z}^{2}}{\omega_{r}^{2}} \frac{T_{d h}}{m_{d h}} \\
+\frac{T_{e}}{T_{d h}} Z_{d h}\left(1-\frac{1}{\delta}-Z_{d l} \frac{n_{d l o}}{n_{i o}}\right)\left(\frac{\pi}{2}\right)^{1 / 2} \frac{\omega_{r}}{k_{z}}\left(\frac{m_{d h}}{T_{d h}}\right)^{1 / 2} e^{-z_{o d h}{ }^{2}} \\
+\frac{T_{e}}{T_{d l}} Z_{d l}\left(1-\frac{1}{\delta}-Z_{d h} \frac{n_{d h o}}{n_{i o}}\right) \frac{2 \gamma}{\omega_{r}} \frac{k_{z}^{2}}{\omega_{r}^{2}} \frac{T_{d l}}{m_{d l}} \\
+\frac{T_{e}}{T_{d l}} Z_{d l}\left(1-\frac{1}{\delta}-Z_{d h} \frac{n_{d h o}}{n_{i o}}\right)\left(\frac{\pi}{2}\right)^{1 / 2} \frac{\omega_{r}}{k_{z}}\left(\frac{m_{d l}}{T_{d l}}\right)^{1 / 2} e^{-z_{o d l}{ }^{2}}
\end{gathered}
$$

Simplifying, rearranging terms and using $\Gamma_{o i} \approx 1$, we get

$$
\begin{aligned}
\left\{( \frac { T _ { e } } { m _ { i } } ) ^ { 1 / 2 } ( \frac { 8 } { \pi } ) ^ { 1 / 2 } ( \frac { k _ { z } ^ { 3 } } { \omega _ { r } ^ { 3 } } ) ( \frac { \gamma } { \omega _ { r } } ) \left[\frac{T_{e}}{m_{d h}} Z_{d h}\left(\delta-1-Z_{d l} \frac{n_{d l o}}{n_{e o}}\right)\right.\right. \\
\left.\left.+\frac{T_{e}}{m_{d l}} Z_{d l}\left(\delta-1-Z_{d h} \frac{n_{d h o}}{n_{e o}}\right)\right]\right\} \\
=-\left(\frac{m_{e}}{m_{i}}\right)^{1 / 2} \Gamma_{o e}-\delta\left(\frac{T_{e}}{T_{i}}\right)^{3 / 2}+\delta\left(\frac{T_{e}}{T_{i}}\right)^{3 / 2} \frac{\vec{k} \cdot \vec{V}_{o}}{\omega_{r}} \\
-\left(\frac{T_{e}}{T_{d h}}\right)^{3 / 2}\left(\frac{m_{d h}}{m_{i}}\right)^{1 / 2} Z_{d h}\left(\delta-1-Z_{d l} \frac{n_{d l o}}{n_{e o}}\right) e^{-z_{o d h}{ }^{2}} \\
-\left(\frac{T_{e}}{T_{d l}}\right)^{3 / 2}\left(\frac{m_{d l}}{m_{i}}\right)^{1 / 2} Z_{d l}\left(\delta-1-Z_{d h} \frac{n_{d h o}}{n_{e o}}\right) e^{-z_{o d l}{ }^{2}}
\end{aligned}
$$


We finally obtain for the growth rate, using the expression (3.70) for $\omega_{r}$,

$$
\begin{aligned}
& \gamma \approx \sqrt{\frac{\pi}{8}}\left\{\frac{C_{s}^{2}\left[\frac{m_{i}}{m_{d h}} Z_{d h}\left(\delta-1-Z_{d l} \frac{n_{d l o}}{n_{e o}}\right)+\frac{m_{i}}{m_{d l}} Z_{d l}\left(\delta-1-Z_{d h} \frac{n_{d h e}}{n_{e o}}\right)\right]^{3 / 2} \omega_{r}}{\left(1+\delta T_{e} / T_{i}+\delta k^{2} \lambda_{d}^{2}\right)^{3 / 2}\left\{\frac{T_{e}}{m_{d h}} Z_{d h}\left(\delta-1-Z_{d l} \frac{n_{d l o}}{n_{e o}}\right)+\frac{T_{e}}{m_{d l}} Z_{d l}\left(\delta-1-Z_{d h} \frac{n_{d h e}}{n_{e o}}\right)\right\}}\right\} \\
& \times {\left[-\left(\frac{m_{e}}{m_{i}}\right)^{1 / 2} \Gamma_{o e}+\delta\left(\frac{T_{e}}{T_{i}}\right)^{3 / 2}\left(\frac{\vec{k} \cdot \vec{V}_{o}}{\omega_{r}}-1\right)\right.} \\
&-\left(\frac{T_{e}}{T_{d h}}\right)^{3 / 2}\left(\frac{m_{d h}}{m_{i}}\right)^{1 / 2} Z_{d h}\left(\delta-1-Z_{d l} \frac{n_{d l o}}{n_{e o}}\right) e^{-z_{o d h}{ }^{2}} \\
&\left.-\left(\frac{T_{e}}{T_{d l}}\right)^{3 / 2}\left(\frac{m_{d l}}{m_{i}}\right)^{1 / 2} Z_{d l}\left(\delta-1-Z_{d h} \frac{n_{d h o}}{n_{e o}}\right) e^{-z_{o d l}{ }^{2}}\right]
\end{aligned}
$$

where

$$
\begin{aligned}
z_{o j}^{2}= & \frac{T_{e}}{2 T_{j}} \frac{m_{j}}{m_{i}}\left\{\frac{m_{i}}{m_{d h}} Z_{d h}\left(\delta-1-Z_{d l} \frac{n_{d l o}}{n_{e o}}\right)\right. \\
& \left.+\frac{m_{i}}{m_{d l}} Z_{d l}\left(\delta-1-Z_{d h} \frac{n_{d h o}}{n_{e o}}\right)\right\} \frac{1}{\left(1+\delta \frac{T_{e}}{T_{i}}+\delta k^{2} \lambda_{d}^{2}\right)}
\end{aligned}
$$

which is the approximate expression of the growth rate for a four-component plasma (two dust species) where $j=d l$ and $d h$ for the light and heavy dust components respectively.

In the following chapters we shall compare numerical solutions of the full dispersion relation with the approximate analytical solutions for plasmas with one and two dust species. 


\section{Chapter 4}

\section{Solution of the Dispersion Relation for a Single Dust Component Plasma}

\subsection{Heavy Dust Component}

We initially consider a three-component plasma comprising of drifting ions, stationary electrons and a single, stationary dust component which we will refer to as being heavy. In Chapter 5 we will apply our analysis to a four-component plasma consisting of two dust components (having different grain mass and charge and hence the distinction between light and heavy). In our analysis we assume that the dust grains are uniform with regard to shape, size, mass and charge and that the grains do not break up or coalesce. All species are fully magnetized. The velocity distributions of all species are isotropic Maxwellians and that of the ion component simply a Doppler shifted Maxwellian. Using standard Vlasov theory and following the method of Gary et al. (1970), the general kinetic dispersion relation for electrostatic fluctuations is 
obtained to be:

$$
\begin{aligned}
0=k^{2} & +\frac{1}{\lambda_{d e}^{2}}\left[1+\frac{\omega}{\sqrt{2} k_{z} C_{e}} \sum_{\mathrm{p}=-\infty}^{\infty} Z\left(z_{p e}\right) \Gamma_{p e}\right] \\
& +\frac{1}{\lambda_{d i}^{2}}\left[1+\frac{\omega-\mathbf{k} \cdot \mathbf{V}_{d i}}{\sqrt{2} k_{z} C_{i}} \sum_{\mathrm{p}=-\infty}^{\infty} Z\left(z_{p i}\right) \Gamma_{p i}\right] \\
& +\frac{1}{\lambda_{d d h}^{2}}\left[1+\frac{\omega}{\sqrt{2} k_{z} C_{d h}} \sum_{\mathrm{p}=-\infty}^{\infty} Z\left(z_{p d h}\right) \Gamma_{p d h}\right]
\end{aligned}
$$

where $e, i$ and $d h$ represent the electron, ion and the heavy dust components respectively. We recall that the wave frequency is given by $\omega=\omega_{r}+\mathrm{i} \gamma$ and $Z$ is the plasma dispersion function. $\Gamma_{p j}=e^{-\alpha_{j}} I_{p}\left(\alpha_{j}\right)$ where $\alpha_{j}=\frac{k_{\perp}^{2} C_{j}^{2}}{\Omega_{j}^{2}}$ for $j=e, i$ and $d h$. $C_{j}=\sqrt{\frac{T_{j}}{m_{j}}}, \Omega_{j}$ and $\lambda_{d j}=\left(T_{j} / 4 \pi n_{j o} q_{j}{ }^{2}\right)^{\frac{1}{2}}$ is the thermal velocity, gyrofrequency and Debye length of species $j$ (where $q_{j}=-e,+e$ and $-Z_{d h} e$ for $j=e, i$ and $d h$ respectively) while $I_{\mathrm{p}}$ is the modified Bessel function of order $\mathrm{p}$.

The components of the wave vector $\mathbf{k}$ are given by $k_{z}\left(k_{\perp}\right)$ along (perpendicular) to the external magnetic field $\mathbf{B}_{o}=B_{o} \hat{\mathbf{z}}$. Also $z_{p e}=\left(\omega-\mathrm{p} \Omega_{e}\right) / \sqrt{2} k_{z} C_{e}$, $z_{p i}=\left(\omega-\mathbf{k} \cdot \mathbf{V}_{d i}-\mathrm{p} \Omega_{i}\right) / \sqrt{2} k_{z} C_{i}$ and $z_{p d h}=\left(\omega-\mathrm{p} \Omega_{d h}\right) / \sqrt{2} k_{z} C_{d h}$. The ion drift velocity is given by $\mathbf{V}_{d i}$. The numerical solution of the dispersion relation is compared with the approximate analytical solution in the next section.

\subsubsection{Numerical Results}

The root-finding technique employed uses Muller's method and deflation. The dispersion relation (4.1) is of the form $D(\omega, k)=0$. The number of roots to be found, initial guess values for the roots and the maximum number of iterations to be performed 
to arrive at each root are supplied by the user. The FORTRAN code then begins by using the initial guess value of the root and by a series of approximations to the true root, attempts to arrive at this root. Once the true root is found, the order of $D(\omega, k)$ is reduced by one (deflation), the guess value of the second root is picked up by the program and the process continues in the usual way until all roots have been found.

In our analysis we assume the drift of the ions to be in the direction of the magnetic field $\mathbf{B}_{\mathbf{o}}$. For simplicity we restrict our analysis to the $y-z$ plane, with $\mathbf{k}=\left(k_{y}, k_{z}\right)=$ $\left(k_{\perp}, k_{z}\right)$. Results are presented in normalized form. Time is normalized by the inverse total ion plasma frequency $\omega_{p i}^{-1}=\left(4 \pi n_{o} e^{2} / m_{i}\right)^{-\frac{1}{2}}$. Speed is normalized by the ion sound speed $C_{s}=\left(T_{e} / m_{i}\right)^{\frac{1}{2}}$, spatial lengths by $\lambda_{d}=\left(T_{e} / 4 \pi n_{o} e^{2}\right)^{\frac{1}{2}}$, densities by the total plasma density $n_{o}$ and temperatures by the electron temperature $T_{e}$; the normalizations having being chosen so as to keep the dust mass $m_{d h}$ and charge $Z_{d h}$ as variable parameters in the numerical work that follows.

Quasi-neutrality at equilibrium requires:

$$
n_{e o}+Z_{d h} n_{d h o}=n_{i o}=n_{o}
$$

where $\left(-e Z_{d h}\right)$ is the uniform charge on each dust particle.

We initially consider wave propagation along $\mathbf{B}_{o}$ i.e. $k_{y} / k=0$ (or $\theta=\sin ^{-1}\left(k_{y} / k\right)=$ $0^{\circ}$ ). It will later become evident that this will correspond to the direction of propagation for which wave growth rate will be a maximum. Standard parameter values chosen are $T_{i}=T_{e}, T_{d h}=0.01 T_{e}, n_{e o}=0.1 n_{o}$ and $Z_{d h}=10^{4}$. The dust grain to ion mass ratio is set to $m_{d h} / m_{i}=10^{12}$ as inferred for the E-ring or G-ring of Saturn 
(Goertz,1989;Rosenberg,1993). The ion to electron mass ratio is set at $m_{i} / m_{e}=1836$ (corresponding to a hydrogen plasma). For these parameters $\omega_{p i} / \omega_{p d h}=1.1 \times 10^{4}$. Assuming grain radii much smaller than the electron Debye length $\left(a<<\lambda_{D e}\right)$, the mass and charge of a dust grain of radius a may be expressed as follows (Meuris et al.,1997):

$$
\begin{aligned}
m(a) & =\frac{4}{3} \pi \rho a^{3} \sim a^{3} \\
q(a) & =4 \pi \varepsilon_{o} \phi_{o} a \sim a
\end{aligned}
$$

where $\varepsilon_{o}$ is the vacuum permittivity, $\rho$ is the mass density of a dust grain and $\phi_{o}$ is the equilibrium surface potential. The charge to mass ratio of a dust grain is thus proportional to $a^{-2}$. In the numerical work that follows, parameters have been selected so as to keep the charge to mass ratio of the dust grains to be proportional to the inverse square of the grain radius. We set $\omega_{p i} / \Omega_{i}=100$, which corresponds to weakly magnetized ions and dust particles.

Figure 4.1 shows that as $V_{d i z} / C_{s}$ increases, there is an increase in the growth rate of the instability. An increase in ion drift velocity results in more free energy becoming available to drive the instability. There is no growth for $V_{d i z}<0.01 C_{s}$ because the beam is not energetic enough to drive the instability. Similar behaviour was observed by Bharuthram et al. (1996). 
The behaviour of the real frequency $\omega_{r}$ for $V_{d i z}=0.3 C_{s}$ is shown in Figure 4.2. The real frequency curves corresponding to the growth rate curves of Figure 4.1 for other beam velocities are not shown because $\omega_{r}$ is found to be independant of ion drift velocity. The behaviour shown in Figure 4.2 is found to be in excellent agreement with the analytical expression obtained by appropriate expansions of the $\mathrm{Z}$ function (Bharuthram et al.,1996), viz.

$$
\omega_{r}^{2}=k_{z}^{2} C_{s}^{2} Z_{d h}(\delta-1) \frac{m_{i}}{m_{d h}} \frac{1}{\left(1+\delta T_{e} / T_{i}+\delta k^{2} \lambda_{d}^{2}\right)}
$$

where $\delta=\left(n_{i o} / n_{e o}=n_{o} / n_{e o}\right)$. The approximations used in deriving equation (4.3) are $|\omega| \ll \Omega_{i}$ and $T_{e} \approx T_{i} \gg T_{d h}$. This expression for a magnetized plasma is similar to that obtained by Rosenberg (1993) for an unmagnetized plasma and that derived earlier by Rao et al. (1990) using a fluid approach. 


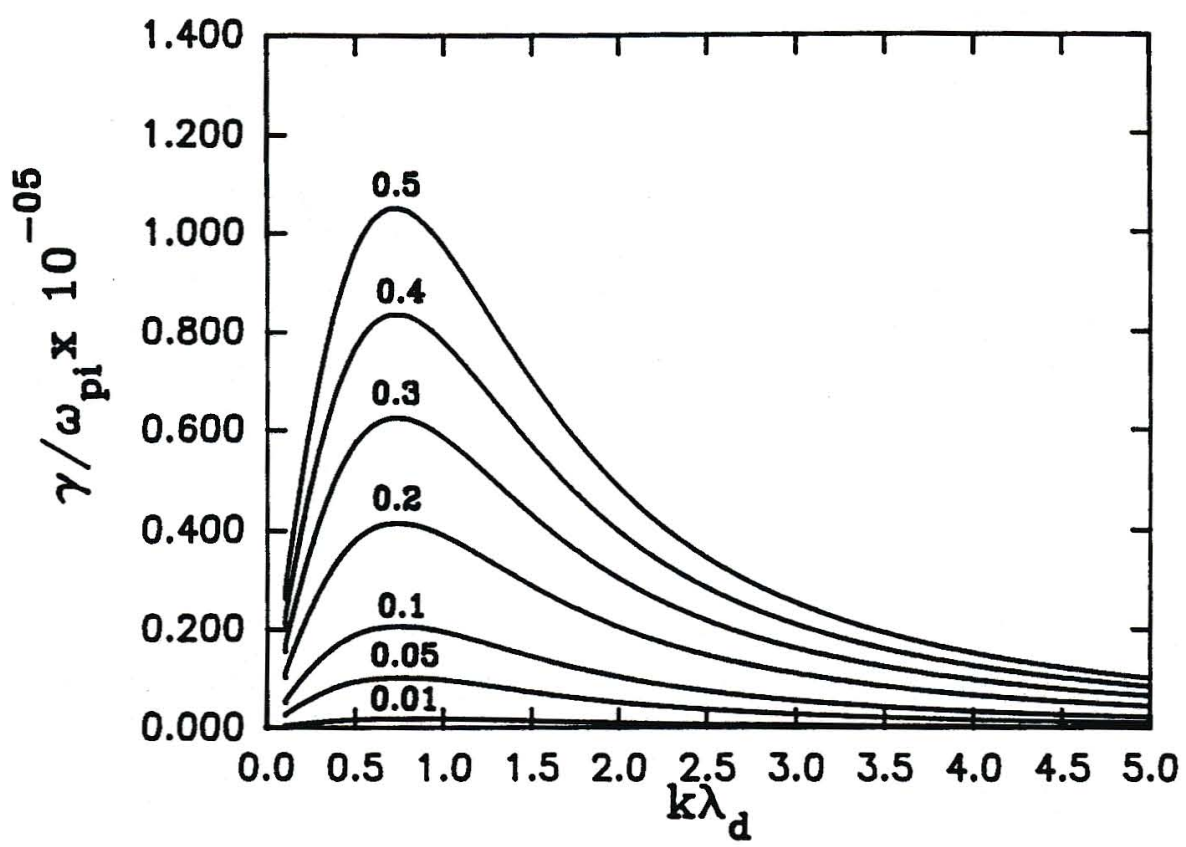

Figure 4.1: Plot of normalized growth rate against normalized wavenumber. The parameter labelling the curves is the normalized ion drift speed $V_{d i z} / C_{s}$. The fixed parameters are $T_{i}=T_{e}, T_{d h}=0.01 T_{e}, n_{e o}=0.1 n_{o}, m_{d h} / m_{i}=10^{12}$ and $Z_{d h}=10^{4}$

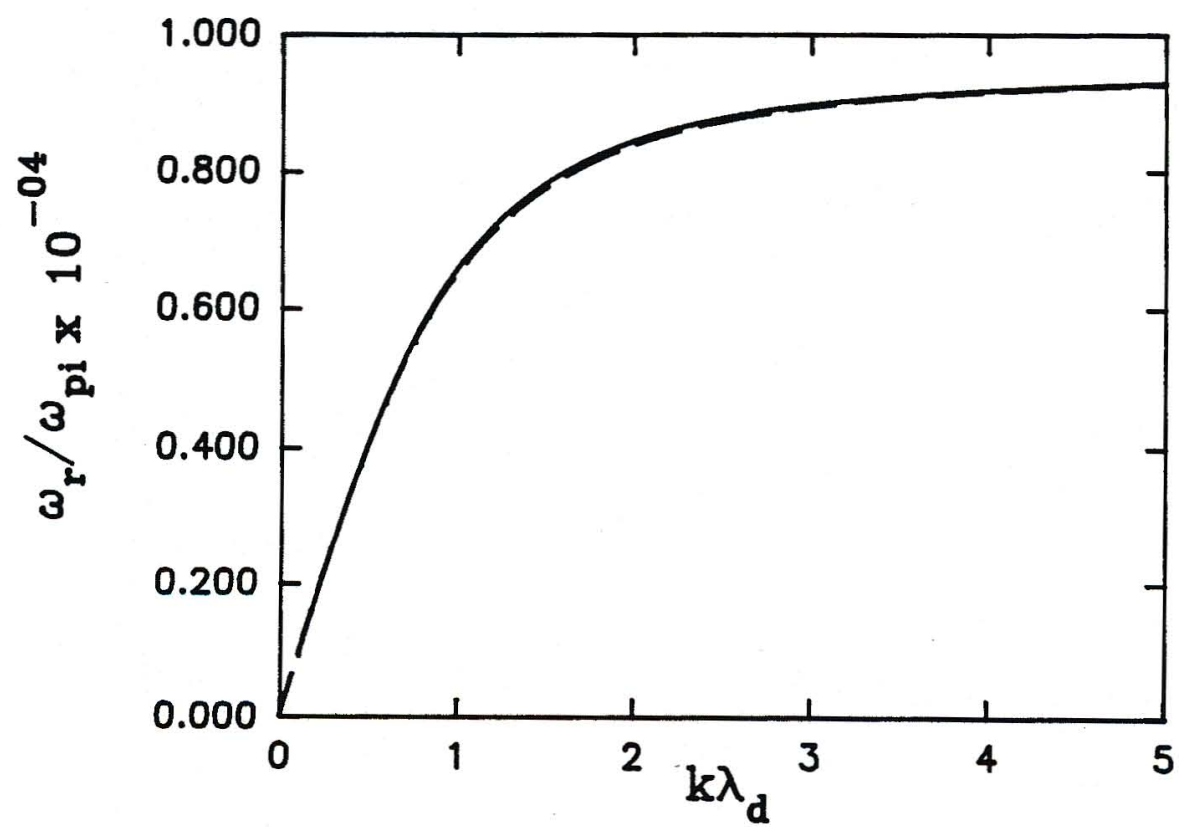

Figure 4.2: Plot of the normalized real frequency versus normalized wavenumber for $V_{d i z}=0.3 C_{s}$. The broken line shows the real frequency calculated from the approximate expression (4.3). All other parameters are as in Figure 4.1 
If the effective temperature of the point particles (electrons and ions) is defined by $Z_{d h}^{2} n_{d h o} / T_{\text {eff }}=n_{e o} / T_{e}+n_{i o} / T_{i}$ (Bharuthram and Shukla,1992), then the analytical expression (4.3) can be rewritten in the form for ion acoustic waves as

$$
\omega_{r}^{2}=\frac{k_{z}^{2} C_{\mathrm{sd}}^{2}}{1+k^{2} \lambda_{\mathrm{deff}}^{2}},
$$

where $C_{\mathrm{sd}}=\left(T_{\mathrm{eff}} / m_{d h}\right)^{\frac{1}{2}}$ and $\lambda_{\mathrm{deff}}=\left(T_{\mathrm{eff}} / 4 \pi n_{d h o} Z_{d h}^{2} e^{2}\right)^{\frac{1}{2}}$.

Figure 4.3 shows that the numerical growth rate is also in excellent agreement with that calculated using the approximate expression

$$
\begin{aligned}
\gamma \approx & \sqrt{\frac{\pi}{8}} \sqrt{Z_{d h}(\delta-1)} \frac{\omega_{r}}{\left(1+\delta T_{e} / T_{i}+\delta k^{2} \lambda_{d}^{2}\right)^{\frac{3}{2}}} \\
& \times\left\{\left[\delta\left(\frac{m_{i}}{m_{d h}}\right)^{\frac{1}{2}}\left(\frac{T_{e}}{T_{i}}\right)^{\frac{3}{2}}\left(\frac{k_{z} V_{d i z}}{\omega_{r}}-1\right)-\Gamma_{o e}\left(\frac{m_{e}}{m_{d h}}\right)^{\frac{1}{2}}\right]\right. \\
& \left.-Z_{d h}(\delta-1)\left(\frac{T_{e}}{T_{d h}}\right)^{\frac{3}{2}} \exp \left[\frac{-T_{e}}{2 T_{d h}} \frac{Z_{d h}(\delta-1)}{\left(1+\delta T_{e} / T_{i}+\delta k^{2} \lambda_{d}^{2}\right)}\right]\right\}
\end{aligned}
$$

which is similar to that obtained by Rosenberg (1993) for an unmagnetized plasma. We see from equation (4.5) that an instability arises $(\gamma>0)$ whenever $V_{d i z}>\omega_{r} / k_{z}$, i.e. the wave phase speed in the direction of the magnetic field sees a positive gradient of the ion velocity distribution. This is a phenomenon known as inverse Landau damping. The remaining terms in (4.5) are the damping terms associated with the electrons and the dust particles. The beam speed has to be sufficiently high enough to overcome the effects of wave damping. Since $\left|\Gamma_{o e}\right| \leq 1$ and $m_{e} \ll m_{d h}$, the damping is negligible for sufficiently large values of $V_{d i z}$. 


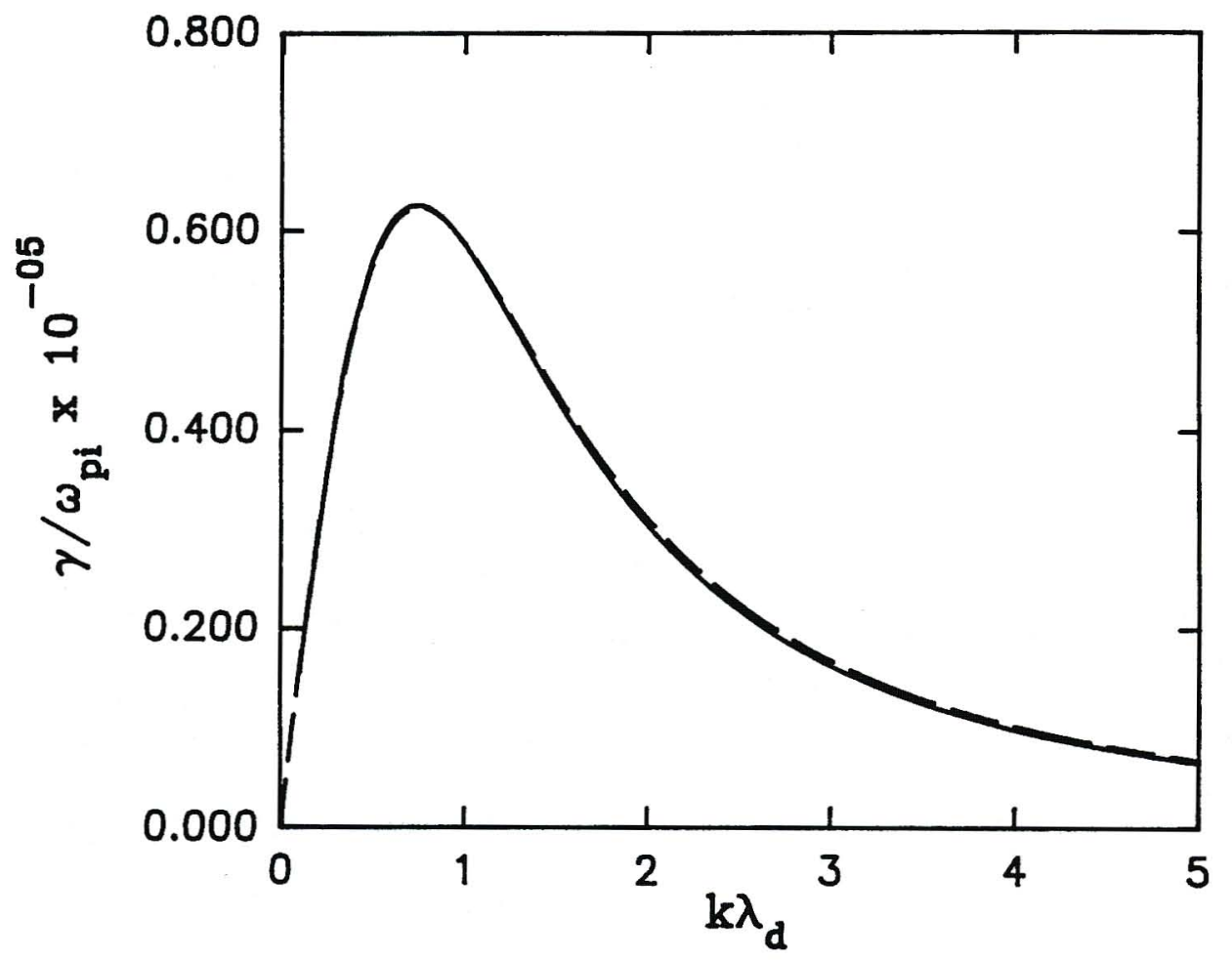

Figure 4.3: Plot of normalized growth rate against normalized wavenumber for $V_{d i z}=$ $0.3 C_{s}$. All other fixed parameters are as in Figure 4.1. The broken line shows the growth rate calculated from the approximate expression (4.5) 
Figure 4.4 shows the effect of the variation of the electron density on the growth rate of the instability. As the equilibrium electron density decreases, the growth rate is found to increase. From this we may conclude that the instability is more easily excited in plasmas with fewer electrons. It is seen in Figure 4.5 that for a fixed $k \lambda_{d}$, the real frequency increases as $n_{e o}$ decreases (the dust density increases). This behaviour is consistent with the analytical expression (4.3) for $\omega_{r}$. A similar trend has been observed for EIC and ion-acoustic modes (D'Angelo,1990).

Figure 4.6 shows the effect of the normalized ion temperature $T_{i} / T_{e}$ on the instability growth rate. As $T_{i} / T_{e}$ increases, the growth rate decreases. For $T_{i} / T_{e}>5.0$ the growth rates become negligible. This reduction in the growth rates with an increase in $T_{i} / T_{e}$ may be explained in terms of the kinetic nature of the dust-acoustic instability. The growth rate $\gamma$ is proportional to the slope $\left(\partial f_{i o} / \partial v_{z}\right)$ of the ion beam velocity distribution. As $T_{i}$ increases the slope of the ion velocity distribution decreases and this 'smaller slope' is seen by a wave propagating along $\vec{B}_{\circ}$ with phase speed $\omega_{r} / k_{z}$. The ion beam velocity distribution is almost flat $\left(\partial f_{i o} / \partial v_{z} \approx 0\right)$ for $T_{i} / T_{e}>5.0$, consequently there is no positive growth. The real frequencies corresponding to the growth rate curves of Figure 4.6 are illustrated in Figure 4.7.

Fig 4.8 illustrates the effect of increasing the equilibrium electron density $n_{e o} / n_{o}$ on the growth rate of the instability. It is seen that wave damping increases with an increase in the concentration of the electrons. 


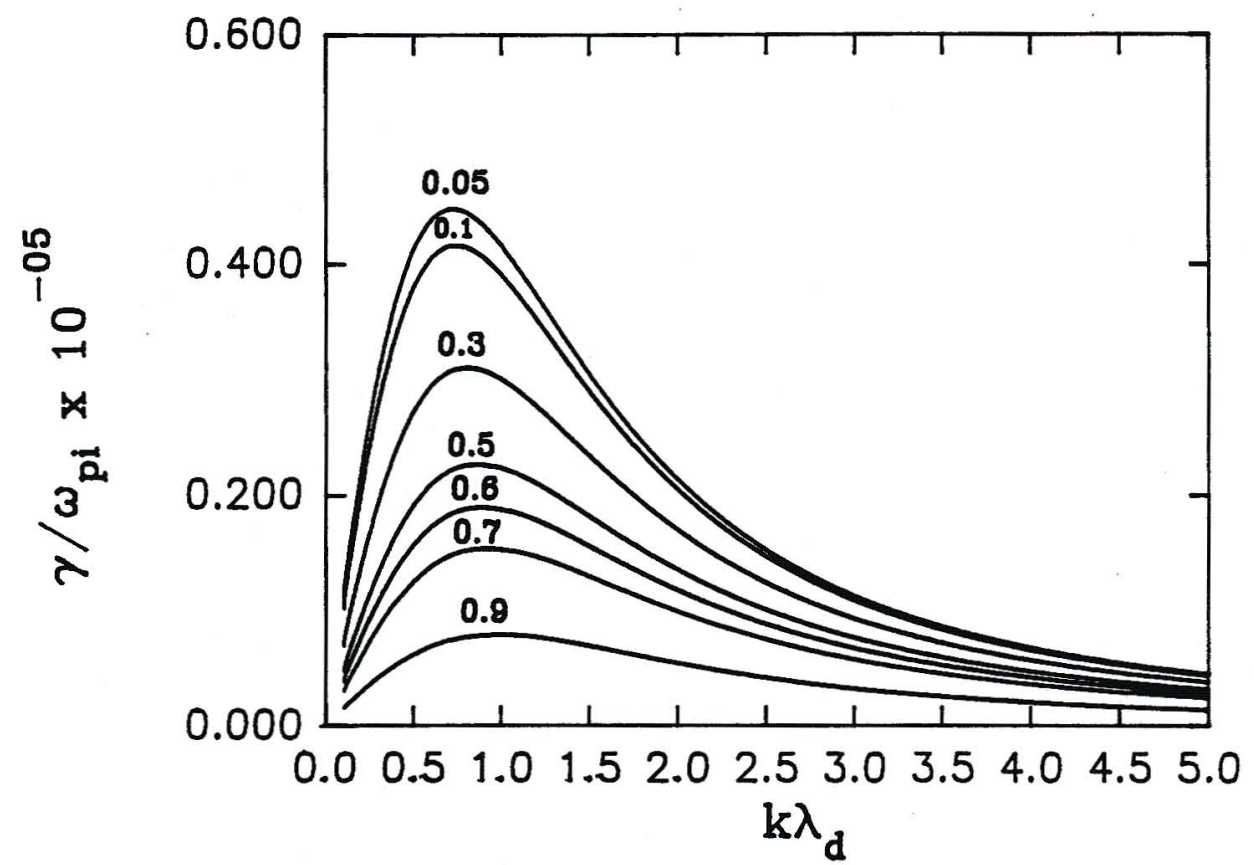

Figure 4.4: Plot of the normalized growth rate against normalized wavenumber for $V_{d i z}=0.2 C_{s}$. The parameter labelling the curves is the normalized electron density $n_{e o} / n_{o}$. All other fixed parameters are as in Figure 4.1

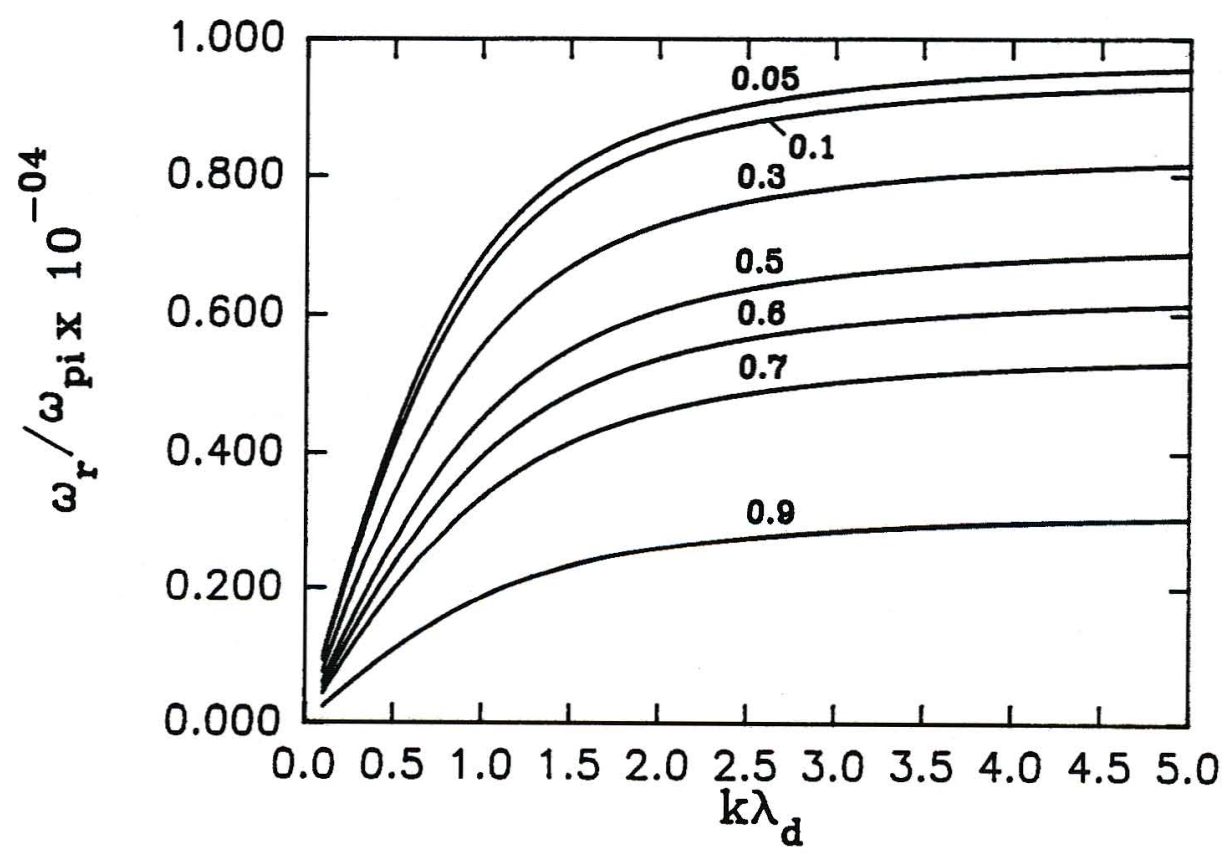

Figure 4.5: Plot of normalized real frequencies corresponding to the growth rate curves in Figure 4.4. The parameter labelling the curves is the normalized equilibrium electron density $n_{e o} / n_{o}$ 


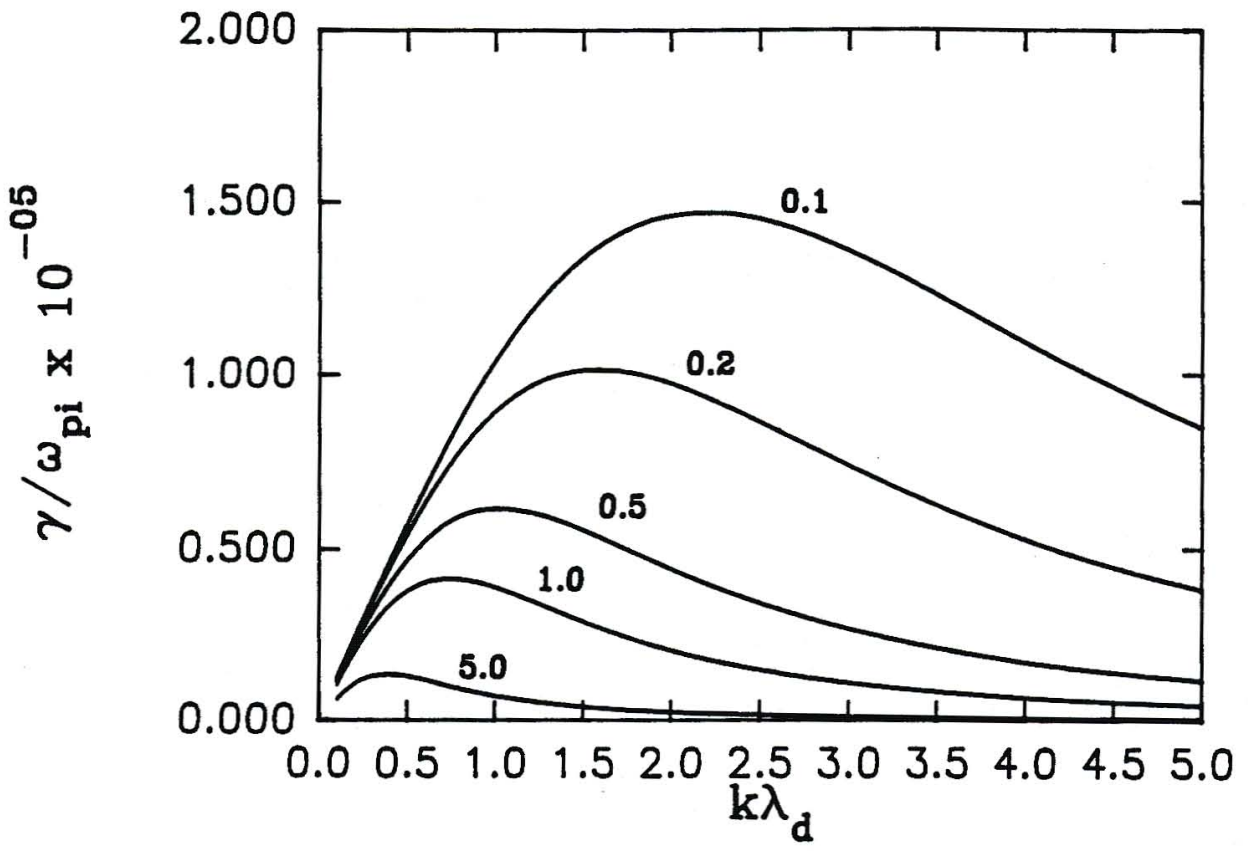

Figure 4.6: Plot of normalized growth rate against normalized wavenumber for $V_{d i z}=$ $0.2 C_{s}$. The parameter labelling the curves is the normalized ion temperature $T_{i} / T_{e}$. All other fixed parameters are as in Figure 4.1

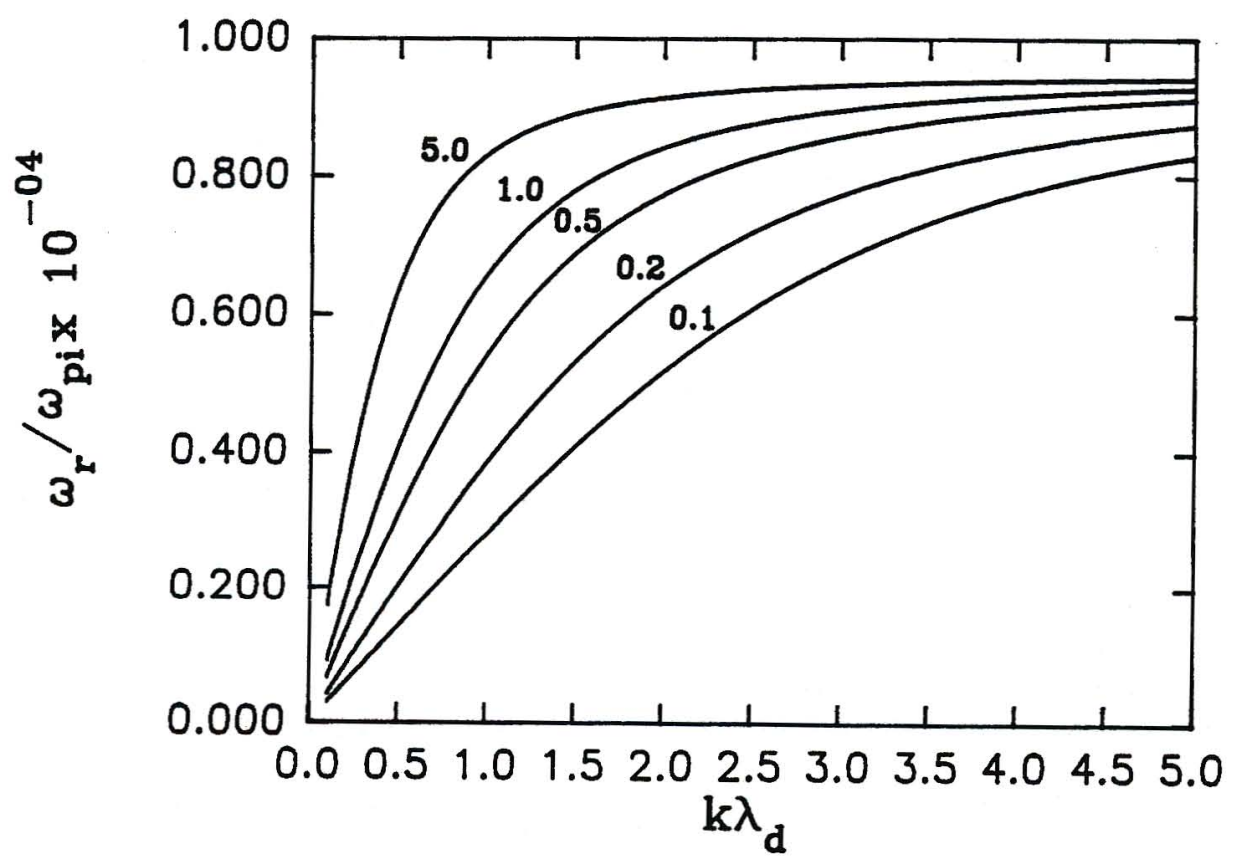

Figure 4.7: Plot of the normalized real frequencies corresponding to the curves in Figure 4.6. The parameter labelling the curves is the normalized ion temperature $T_{i} / T_{e}$ 
(a)

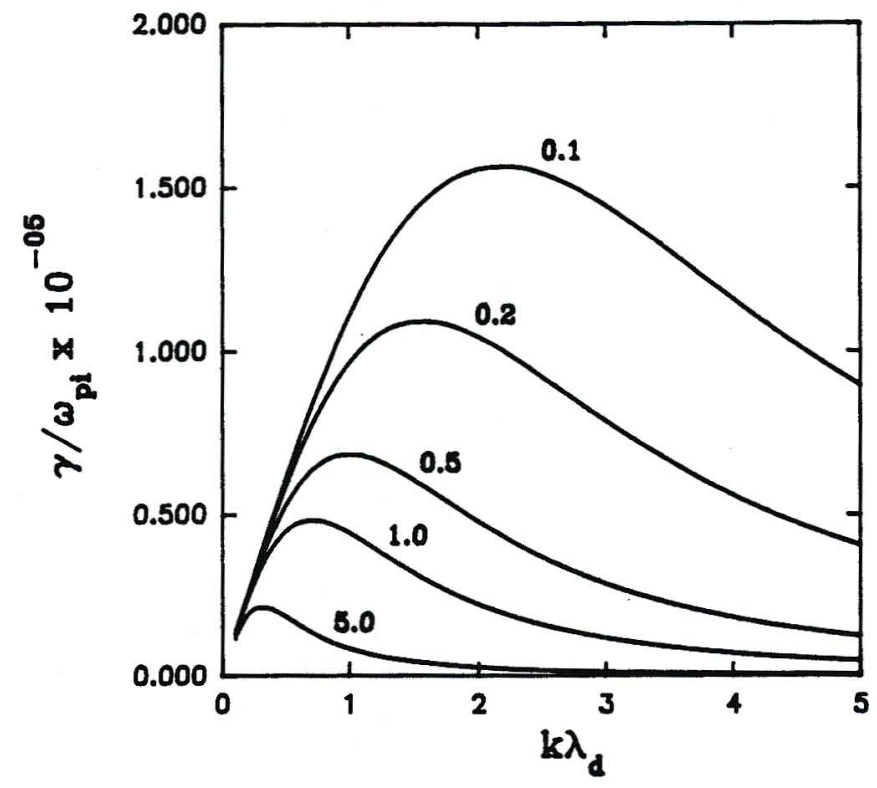

(c)

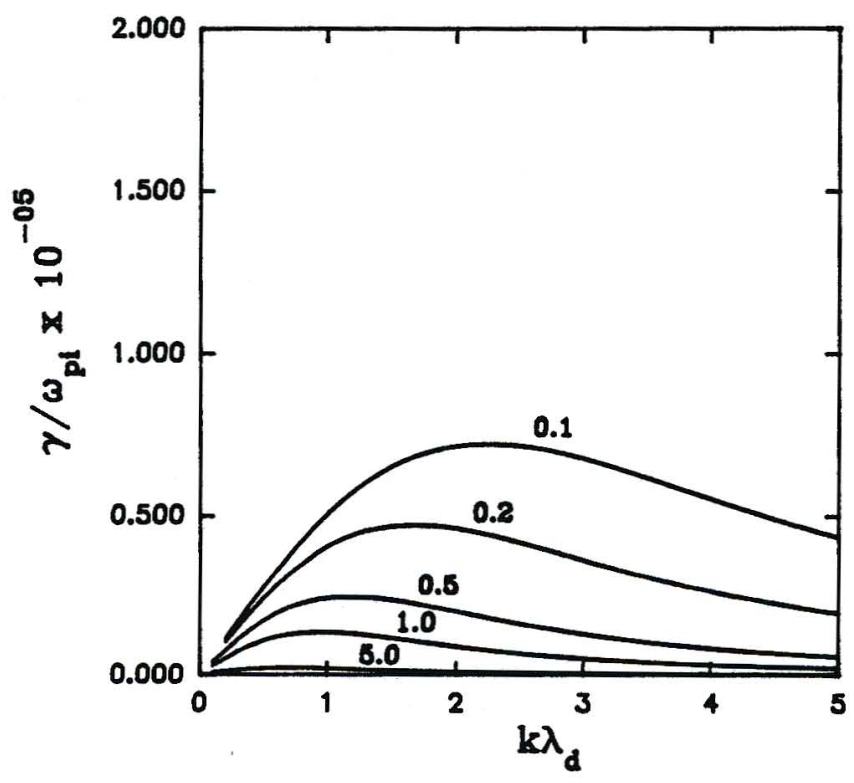

(b)

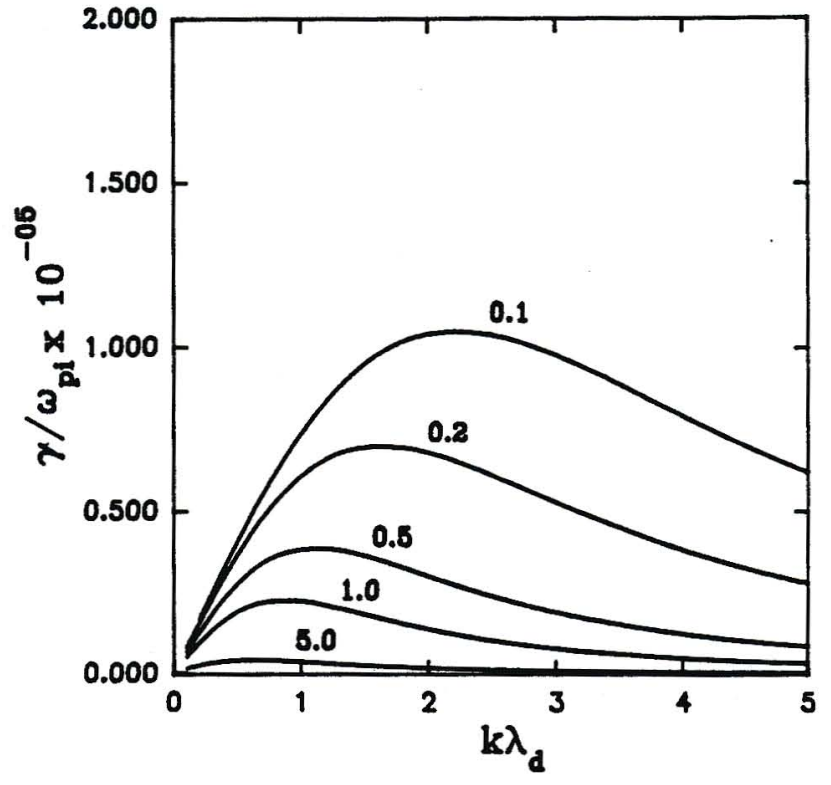

(d)

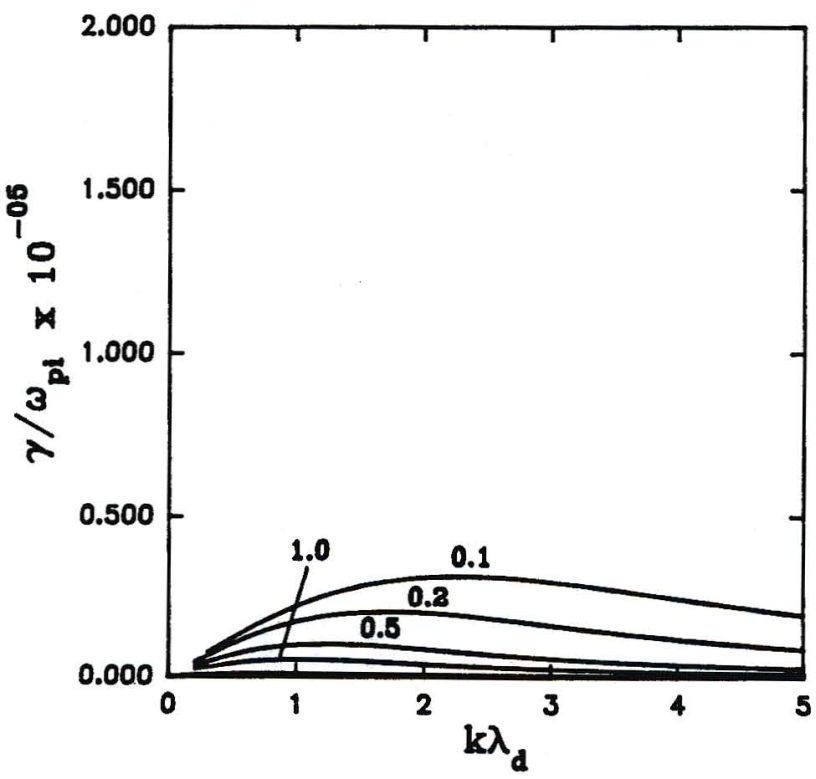

Figure 4.8: Plot of the normalized growth rates as a function of normalized wave number for different values of the electron density $n_{e o} / n_{o}$ : (a) $n_{e o}=0.00 n_{o}$, (b) $n_{e o}=0.5 n_{o}$, (c) $n_{e o}=0.75 n_{o}$ and (d) $n_{e o}=0.95 n_{o}$. The parameter labelling the curves is $T_{i} / T_{e}$. All other parameters are as in Figure 4.6. 
We finally study the effect of the magnetic field strength on the instability. It is seen in Figure 4.9 that with an increase in the magnitude of $B_{o}\left(\omega_{p i} / \Omega_{i}\right.$ decreasing $)$, the ion magnetization increases, the range of propagation angles for positive growth widens and the growth rates increase. As the magnetic field strength increases, the motion of the ions becomes increasingly restricted by the magnetic field lines. The ions are not so 'free' to move to shortout potential perturbations of a wave propagating in a direction oblique to $\mathbf{B}_{\mathbf{o}}$. As a result, wave growth rate is enhanced. For a weakly magnetized plasma, the instability is restricted to a narrow cone of propagation angles about the beam direction displaying similar behaviour to that of an unmagnetized plasma. The growth rate $\gamma$ is maximum for propagation along $\mathbf{B}_{\mathbf{o}}$ since this is the direction of propagation for which the wave can gain maximum energy from the ion beam.

The real frequency (1) and growth rate (2) curves for selected oblique angles of propagation are shown in Figures $4.10,4.11$ and 4.12 corresponding to angles $\theta=$ $\sin ^{-1}\left(k_{y} / k\right)=45^{\circ}, 30^{\circ}$ and $72^{\circ}$ respectively. Results differ from Bharuthram et al. (1996) in that the instability becomes the dust-modified two-stream instability (DMTSI) (Rosenberg and Krall,1995) for $\omega_{p i} / \Omega i>0.01$ i.e. when the ions become less strongly magnetized. 


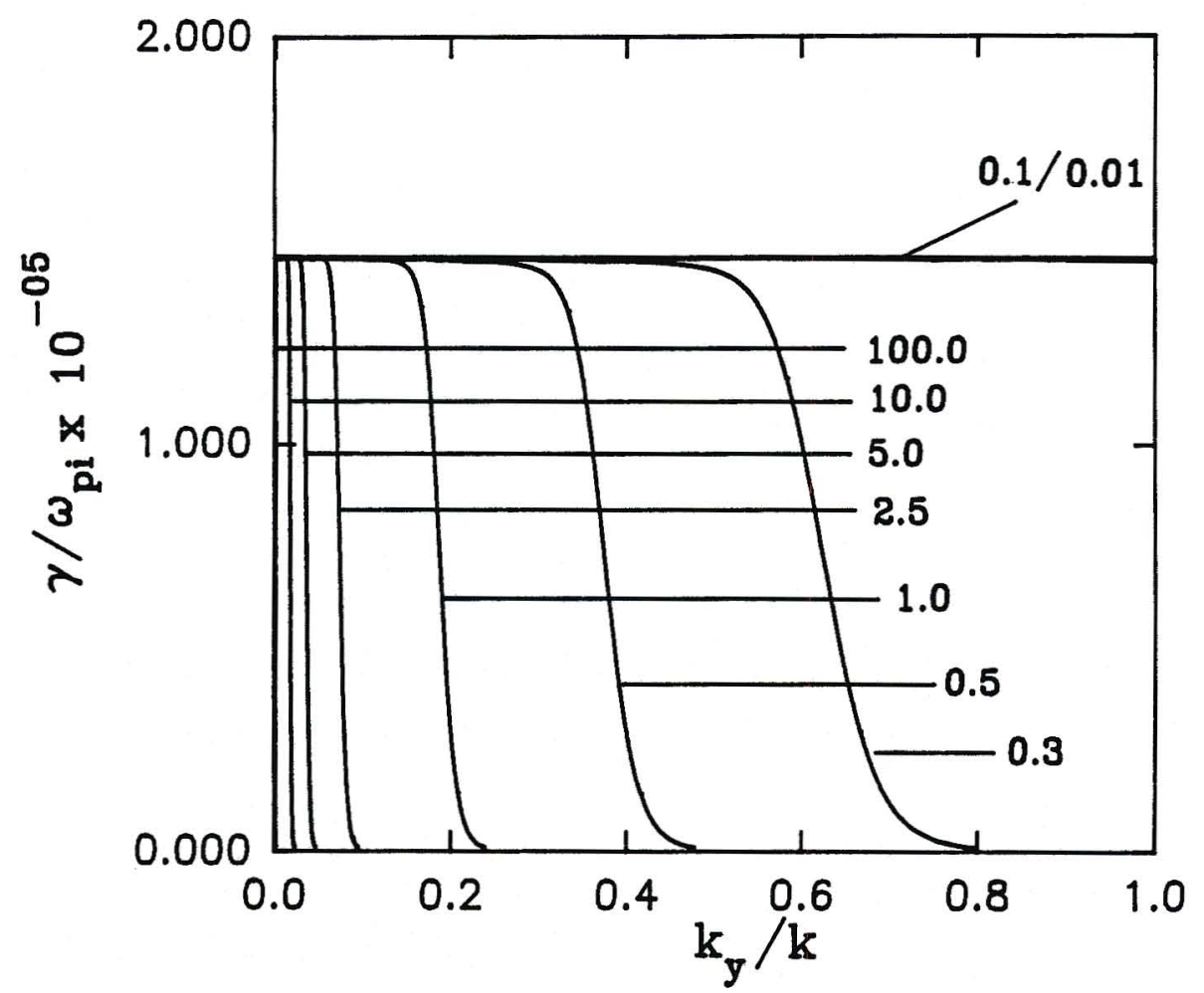

Figure 4.9: Plot of normalized growth rate as a function of $k_{y} / k$. The parameter labelling the curves is $\omega_{p i} / \Omega_{i}$. Fixed parameters are $V_{d i z}=0.2 C_{s}, k \lambda_{d}=2.4$ and $T_{i}=$ $0.1 T_{e}$. All other parameters are as in Figure 4.1 
(1)

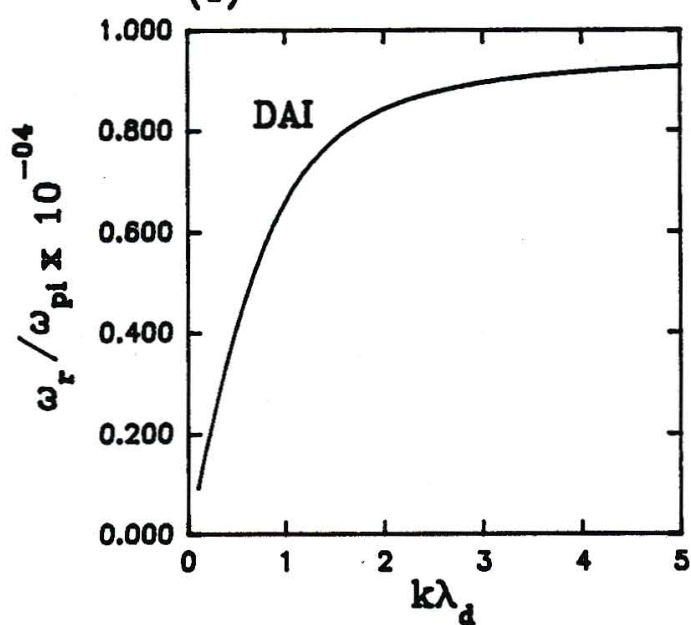

(1)

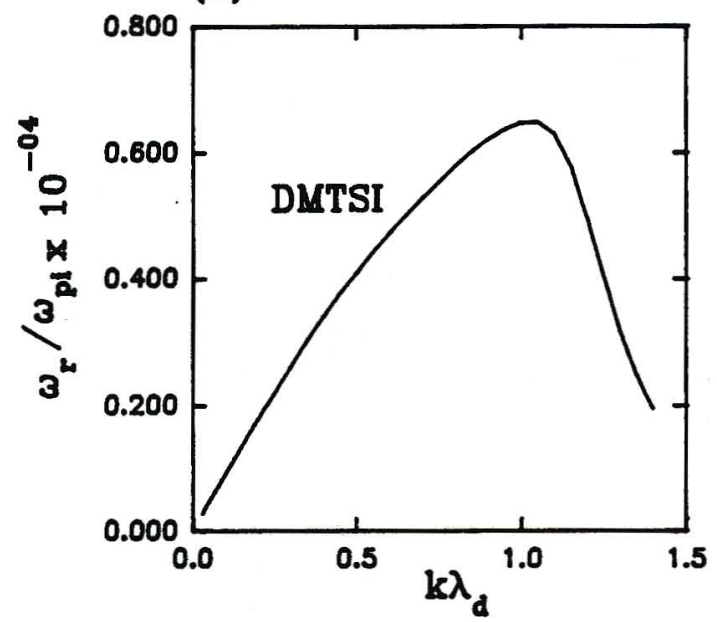

(1)

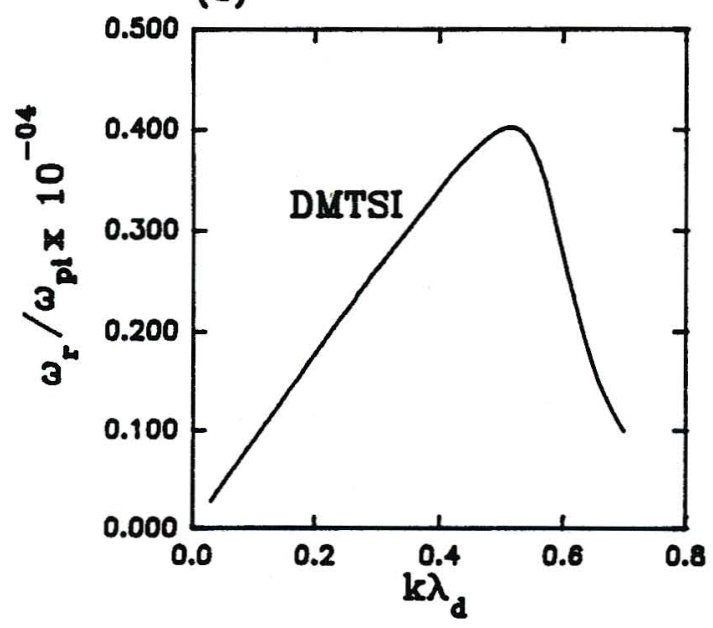

(a)

(2) 48

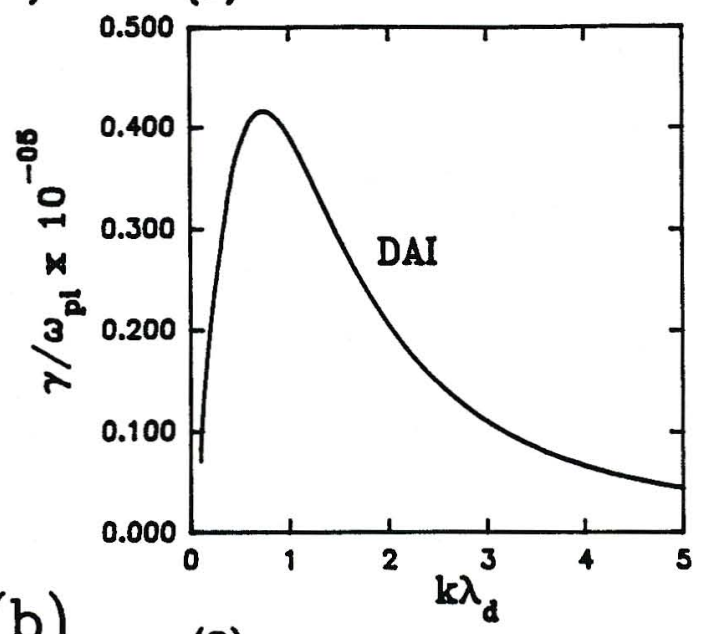

(2)

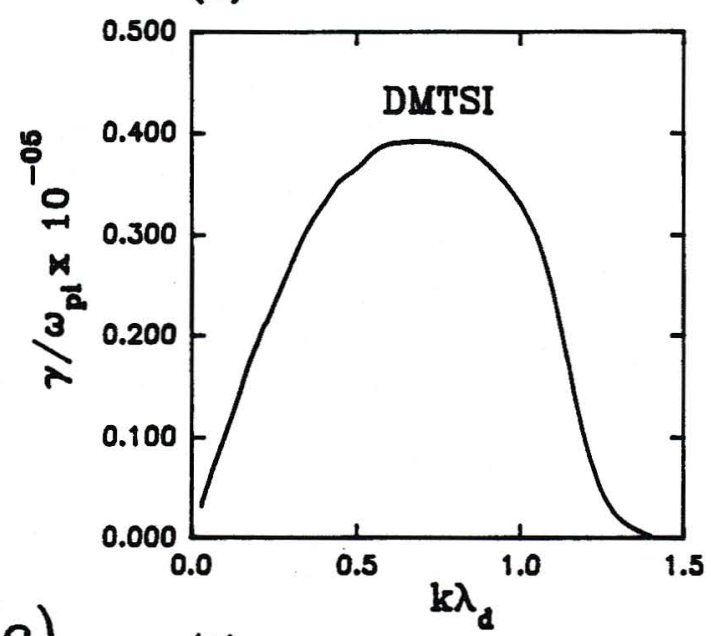

(2)

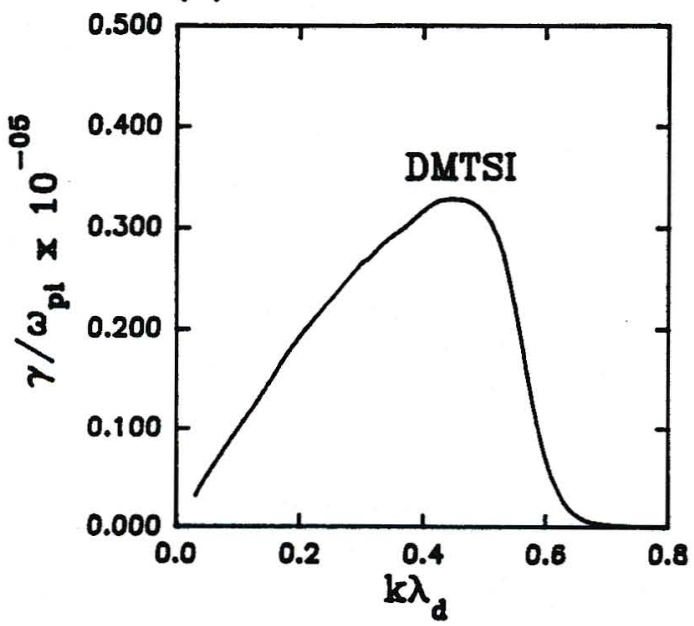

Figure 4.10: Plot of the normalized real frequency (1) and growth rate (2) versus $k \lambda_{d}$ for oblique angles of propagation relative to $\mathbf{B}_{\mathbf{o}}$. Here $T_{i}=T_{e}$ and $k_{y} / k=$ $0.707\left(\theta=45^{\circ}\right)$ for various values of $\omega_{p i} / \Omega_{i}$ : (a) $\omega_{p i} / \Omega_{i}=0.01$, (b) $\omega_{p i} / \Omega_{i}=0.5$ and (c) $\omega_{p i} / \Omega_{i}=1.0$. All other fixed parameters are as in Figure 4.9 
(1)

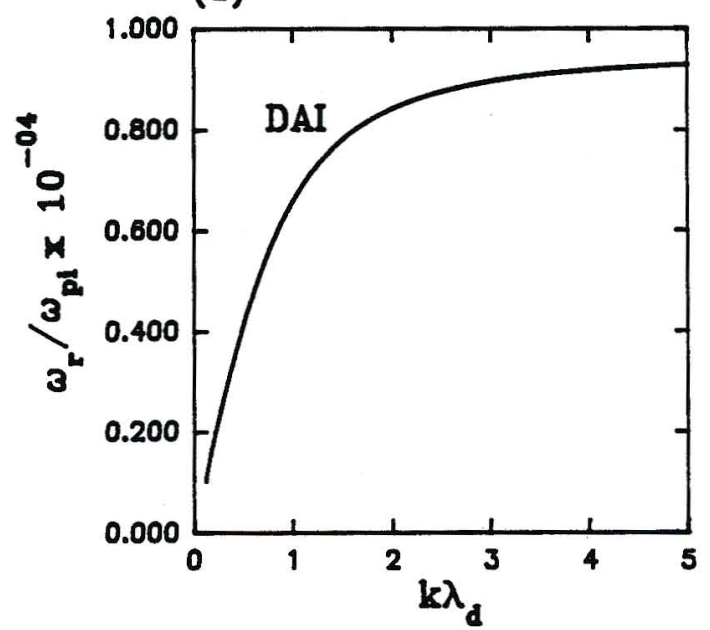

(1)

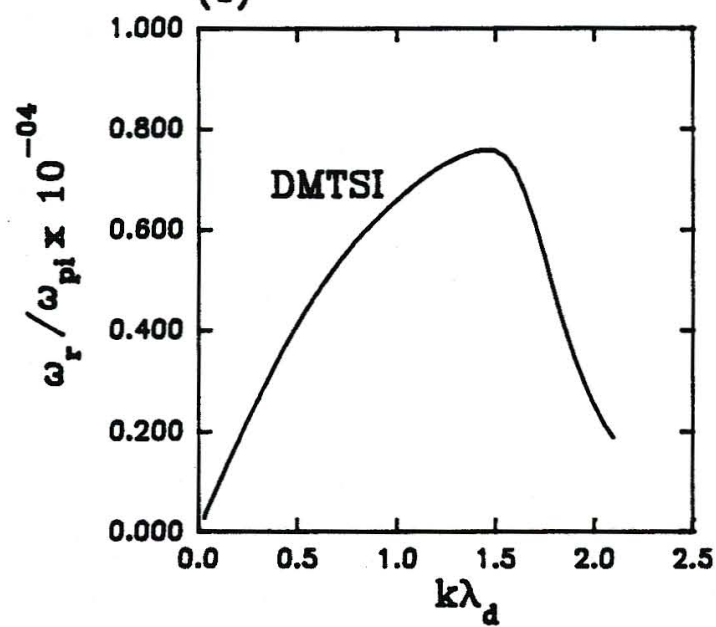

(1)

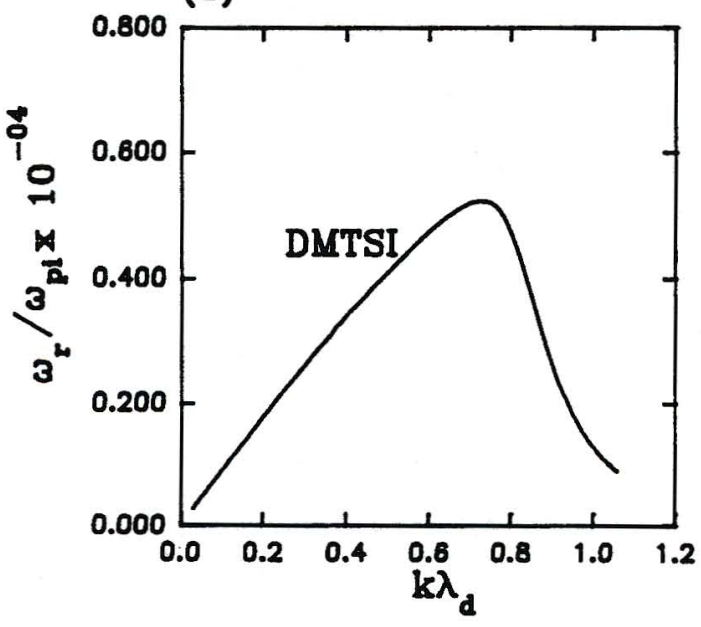

(a)

(2)

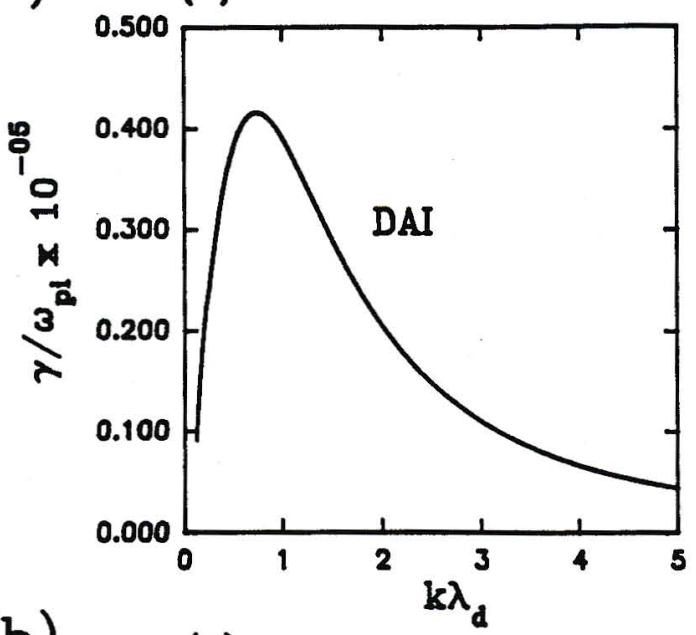

(2)

(b)

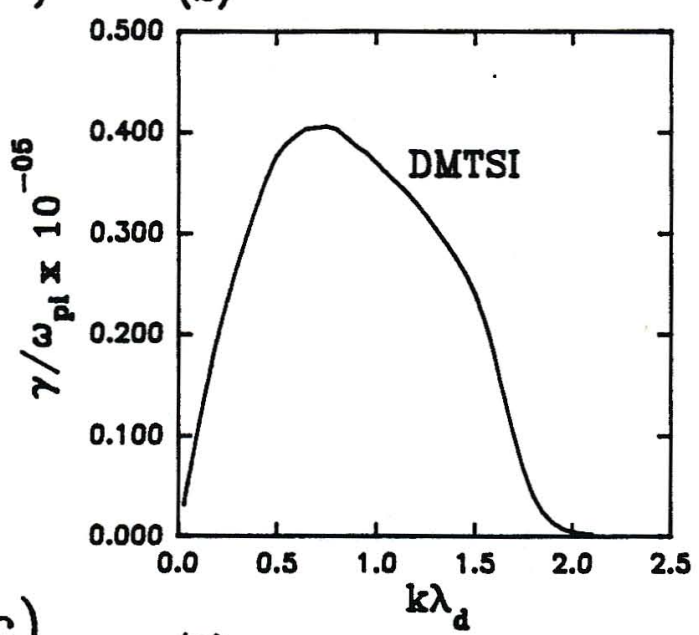

(c)

(2)

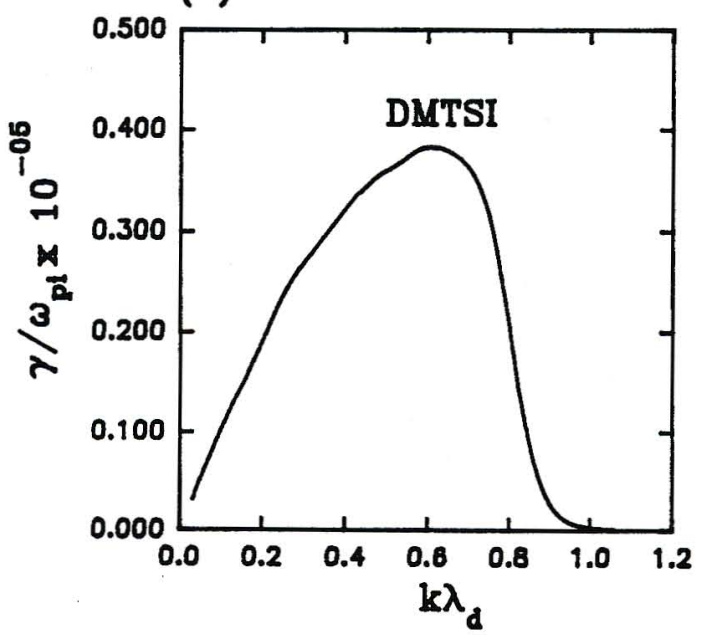

Figure 4.11: Plot of the normalized real frequency (1) and growth rate (2) versus $k \lambda_{d}$. Here $k_{y} / k=0.5\left(\theta=30^{\circ}\right)$ for various values of $\omega_{p i} / \Omega_{i}$ : (a) $\omega_{p i} / \Omega_{i}=0.01$, (b) $\omega_{p i} / \Omega_{i}=0.5$ and (c) $\omega_{p i} / \Omega_{i}=1.0$. All other fixed parameters are as in Figure 4.10 
(1)

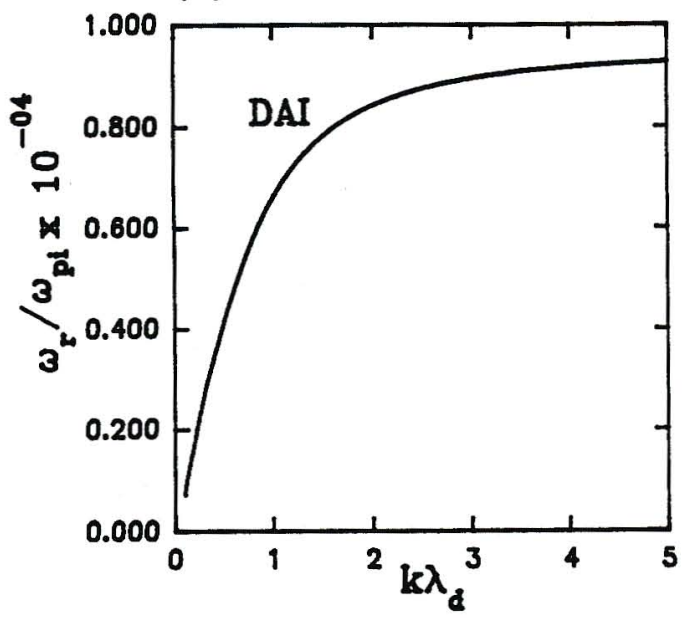

(1)

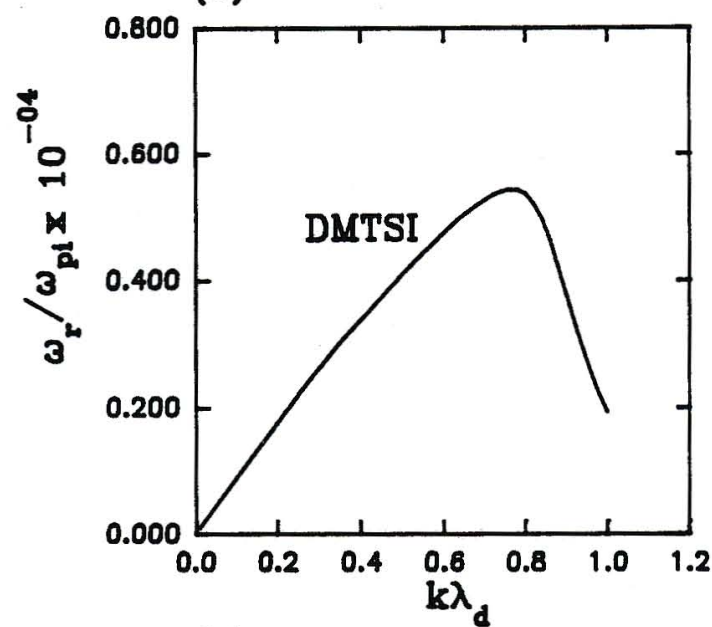

(1)

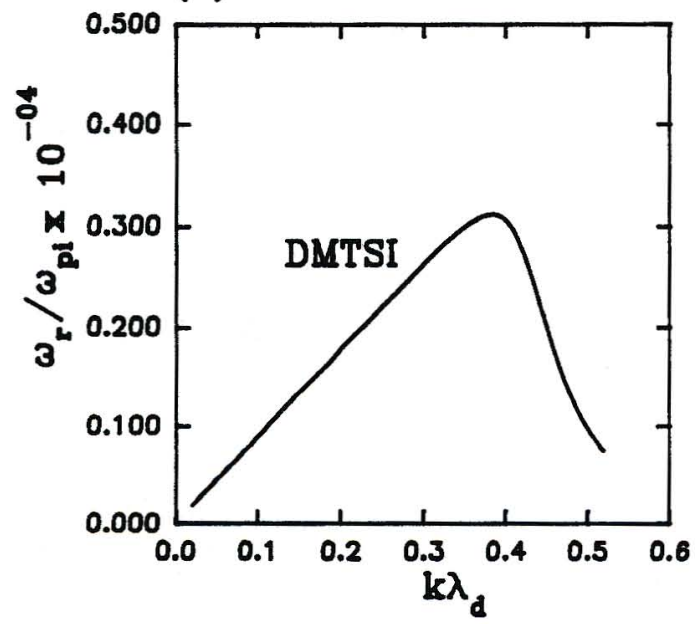

(a)

(2)

(b)

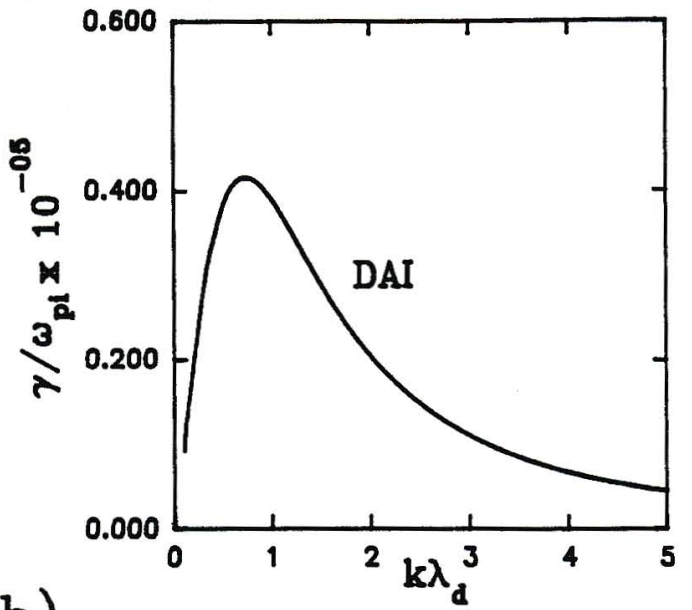

(2)

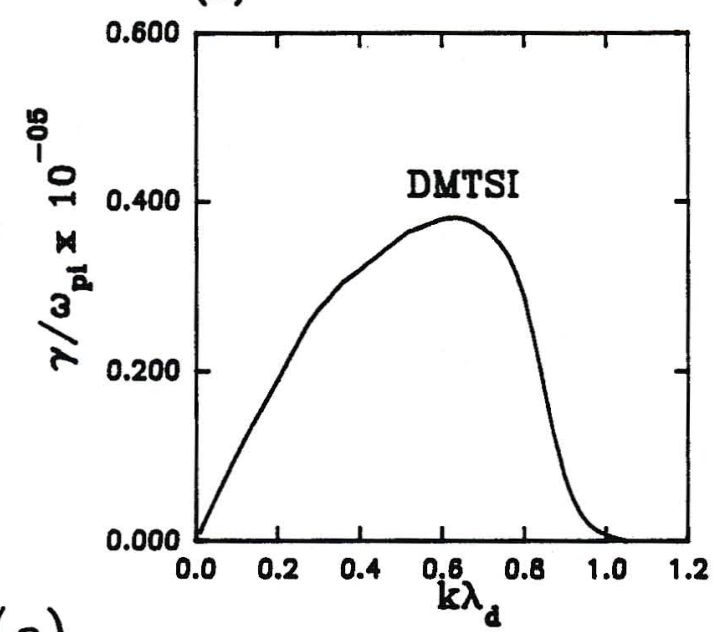

(2)

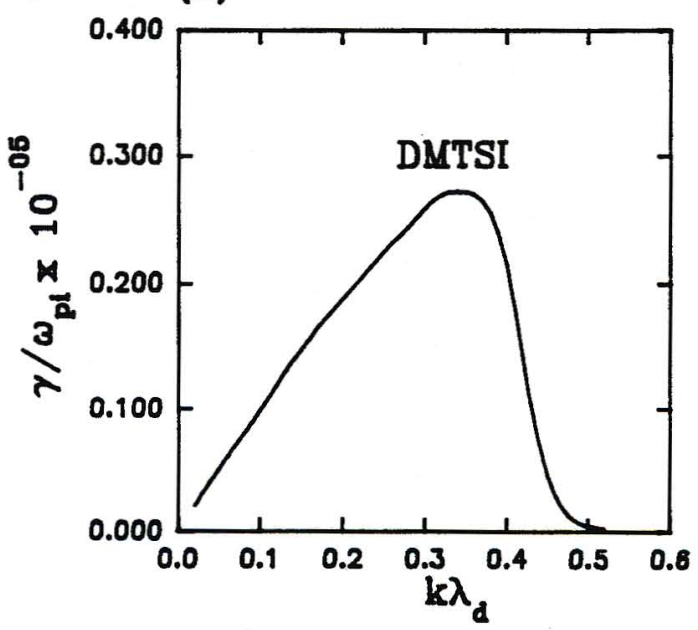

Figure 4.12: Plot of normalized real frequency (1) and growth rate (2) versus $k \lambda_{d}$. Here $k_{y} / k=0.95\left(\theta=72^{\circ}\right)$ for various values of $\omega_{p i} / \Omega_{i}$ : (a) $\omega_{p i} / \Omega_{i}=0.01$, (b) $\omega_{p i} / \Omega_{i}=0.5$ and (c) $\omega_{p i} / \Omega_{i}=1.0$. All other fixed parameters are as in Figure 4.10 


\subsection{Light Dust Component}

We now consider a three-component plasma consisting of drifting ions, stationary electrons and a single stationary dust component which we will refer to as being light due to the smaller dust to ion mass ratio of the individual grains when compared to the heavier dust component in section 4.1. All species are again fully magnetized. The velocity distributions of all species are the same as in section 4.1. The general kinetic dispersion relation similar to that given in section 4.1 is given by:

$$
\begin{aligned}
0=k^{2} & +\frac{1}{\lambda_{d e}^{2}}\left[1+\frac{\omega}{\sqrt{2} k_{z} C_{e}} \sum_{\mathrm{p}=-\infty}^{\infty} Z\left(z_{p e}\right) \Gamma_{p e}\right] \\
& +\frac{1}{\lambda_{d i}^{2}}\left[1+\frac{\omega-\mathbf{k} \cdot \mathbf{V}_{d i}}{\sqrt{2} k_{z} C_{i}} \sum_{\mathrm{p}=-\infty}^{\infty} Z\left(z_{p i}\right) \Gamma_{p i}\right] \\
& +\frac{1}{\lambda_{d d l}^{2}}\left[1+\frac{\omega}{\sqrt{2} k_{z} C_{d l}} \sum_{\mathrm{p}=-\infty}^{\infty} Z\left(z_{p d l}\right) \Gamma_{p d l}\right]
\end{aligned}
$$

where $e, i$ and $d l$ represent the electron, ion and light dust components respectively. All symbols have their usual meaning as defined in section 4.1. This dispersion relation is numerically solved and comparison is made with the approximate analytical solution.

\subsubsection{Numerical Results}

Here again we assume the ion drift to be in the direction of the magnetic field $\mathbf{B}_{\mathbf{o}}$. Results are presented in normalized form. Normalizations are the same as in section 4.1. Quasi-neutrality at equilibrium requires:

$$
n_{e o}+Z_{d l} n_{d l o}=n_{i o}=n_{o}
$$


where $\left(-e Z_{d l}\right)$ is the uniform charge on each dust particle of the lighter species.

In the numerical work that follows the standard parameter values used are $T_{i}=T_{e}$, $T_{d l}=0.01 T_{e}, n_{e o}=0.1 n_{o}$ and $Z_{d l}=10^{2}$. The dust grain to ion mass ratio is set to $m_{d l} / m_{i}=10^{6}$. We again consider a hydrogen plasma $\left(m_{i} / m_{e}=1836\right)$. For the chosen parameters $\omega_{p i} / \omega_{p d l}=1.1 \times 10^{2}$. We again consider weakly magnetized ions and dust particles $\left(\omega_{p i} / \Omega_{i}=100\right)$.

It is seen in Figure 4.13 that as the normalized ion drift velocity $V_{d i z} / C_{s}$ increases, there is a corresponding increase in the growth rate of the instability. This is due to more free energy becoming available to drive the instability. For very low ion drift speeds $V_{d i z}<0.01 C_{s}$, there is no growth as was the case for the single heavy dust constituent plasma in the previous section.

As observed for the single heavy dust constituent plasma, the real frequency is again found to be in excellent agreement with the analytical expression (4.3) of the real frequency for the three-component plasma given in the previous section. The numerical growth rate is also found to be in excellent agreement with that calculated using the approximate expression (4.5) as given in section 4.1.

As was discussed for the single heavy dust constituent plasma, the dust-acoustic mode is unstable whenever the wave sees a positive slope of the ion velocity distribution, i.e. at any particular wave speed, the ions that are travelling slightly faster than the wave can impart more energy to the wave than those travelling slightly slower, and hence resulting in its amplitude to grow in time (inverse Landau damping). 


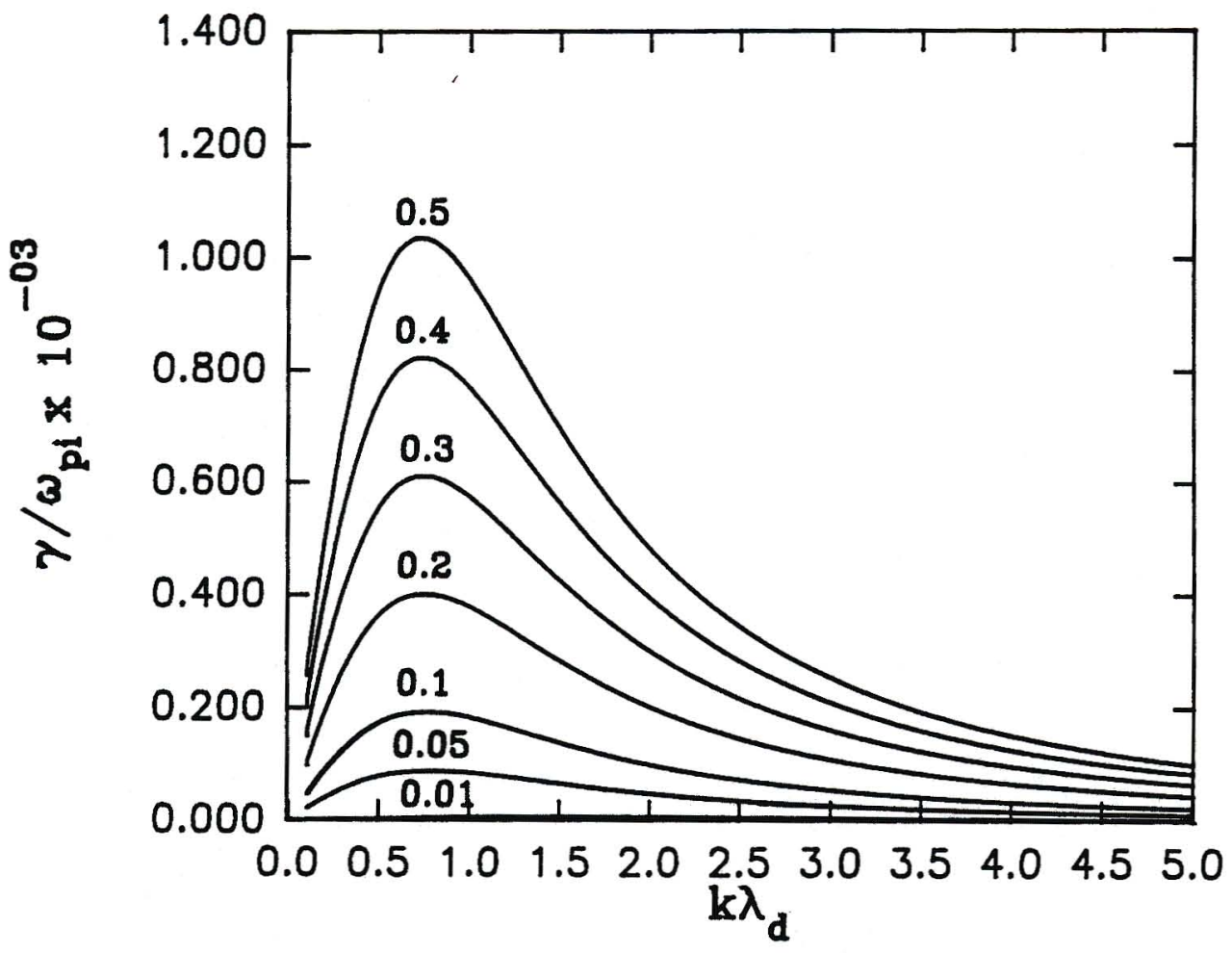

Figure 4.13: Plot of the normalized growth rate against normalized wavenumber. The parameter labelling the curves is the normalized ion drift speed $V_{d i z} / C_{s}$. The fixed parameters are $T_{i}=T_{e}, T_{d l}=0.01 T_{e}, n_{e o}=0.1 n_{o}, m_{d l} / m_{i}=10^{6}$ and $Z_{d l}=10^{2}$ 
In Figure 4.14 we see the effect of the variation of the electron density on the growth rate of the instability. The growth rate is found to increase as the concentration of the electrons decreases. This is consistent with the results for the single heavy dust constituent plasma. We see that the wave frequency (Figure 4.15) and growth rate (Figure 4.14) are about two orders of magnitude higher than those for the heavy dust component plasma of the previous section. This is due to the dust grains being lighter and hence fluctuations in potential would occur on much shorter time scales than those associated with the more massive, heavier dust grains. It is observed in Figure 4.15 that for a fixed $k \lambda_{d}$, the normalized real frequency $\omega_{r}$ increases as $n_{e o} / n_{o}$ decreases as was the case for the three-component plasma in the previous section.

Figure 4.16 illustrates the effect of the normalized ion temperature $T_{i} / T_{e}$ on the growth rate of the instability. As $T_{i} / T_{e}$ increases, the growth rate decreases. Results are consistent with the findings for a single heavy dust species plasma. The lowering of the growth rates with an increase in $T_{i} / T_{e}$ may again be explained in terms of the kinetic nature of the dust-acoustic instability. As $T_{i}$ increases, the ion velocity distribution 'flattens' and thus a 'smaller slope' is seen by a wave propagating along Bo with phase speed $\omega_{r} / k_{z}$. The real frequency curves are depicted in Figure 4.17.

Figure 4.18 illustrates that the Landau damping increases with an increase in the normalized electron density $n_{e o} / n_{o}$. This is consistent with the findings for the heavy dust constituent plasma. 


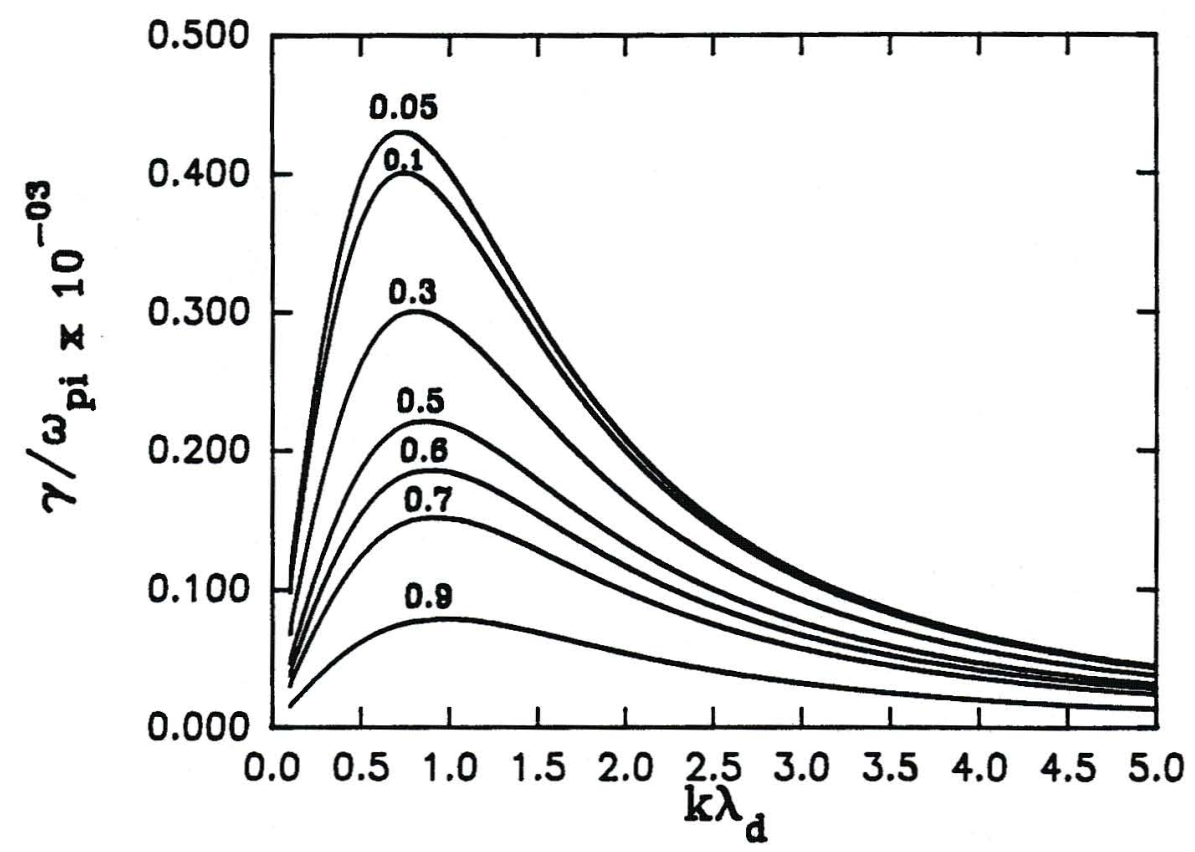

Figure 4.14: Plot of normalized growth rate against normalized wavenumber for $V_{d i z}=$ $0.2 C_{s}$. The parameter labelling the curves is the normalized electron density $n_{e o} / n_{o}$. All other fixed parameters are as in Figure 4.13

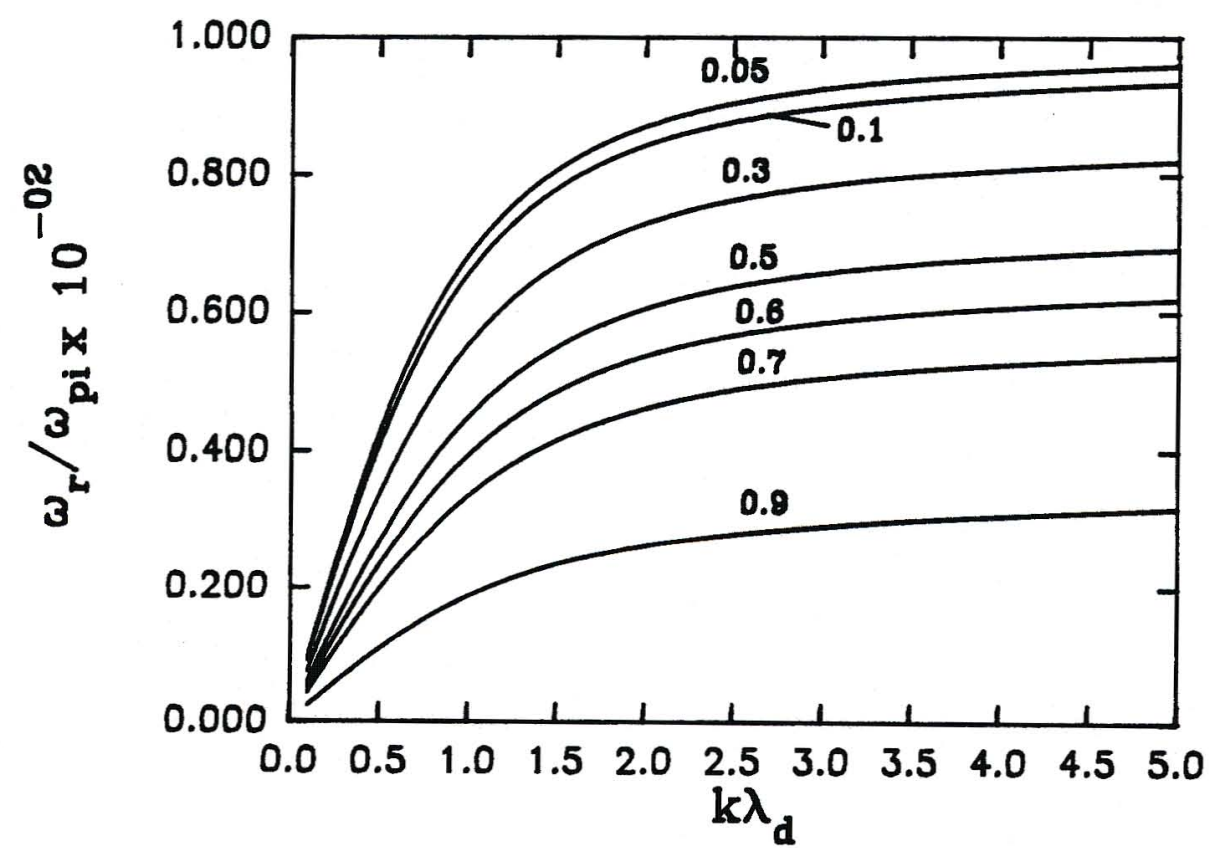

Figure 4.15: Plot of normalized real frequencies corresponding to the growth rate curves in Figure 4.14. The parameter labelling the curves is $n_{e o} / n_{o}$ 


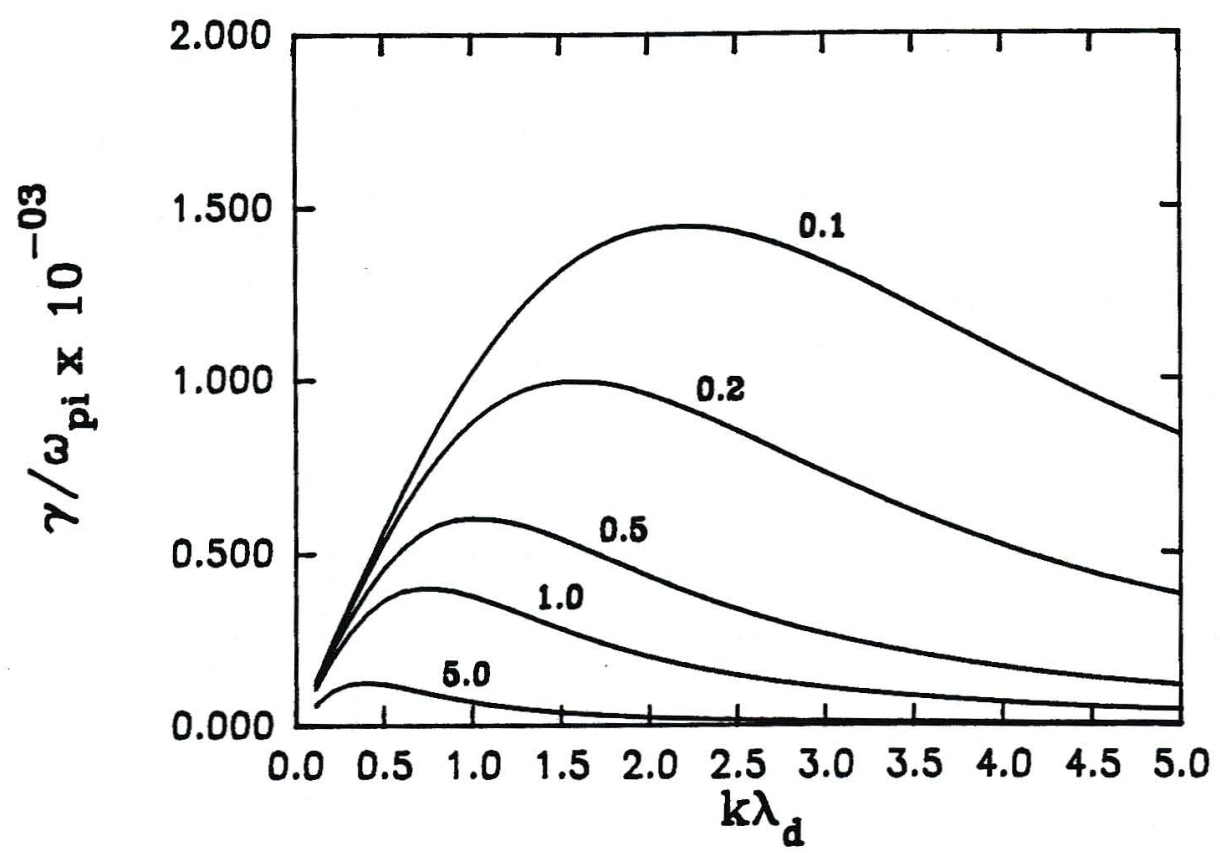

Figure 4.16: Plot of the normalized growth rate against normalized wavenumber for $V_{d i z}=0.2 C_{s}$. The parameter labelling the curves is the normalized ion temperature $T_{i} / T_{e}$. All other fixed parameters are as in Figure 4.13

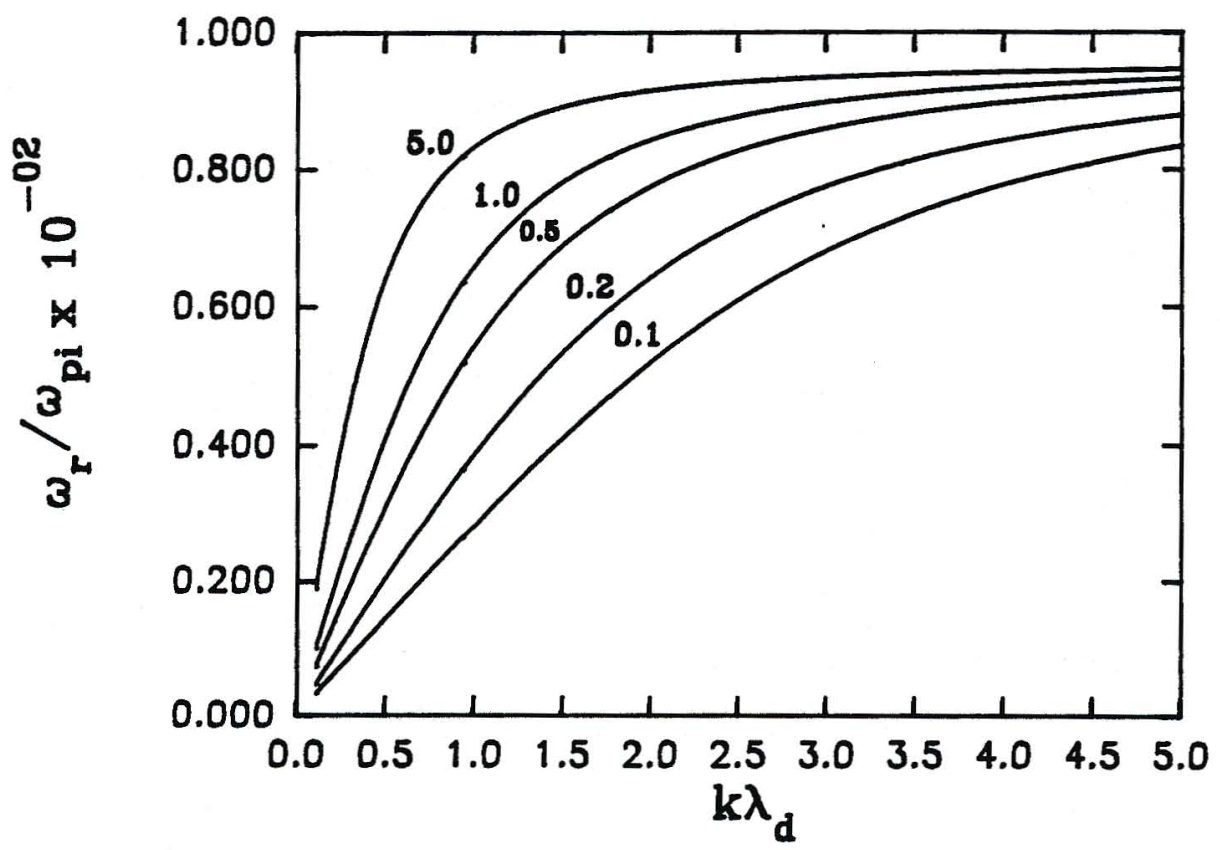

Figure 4.17: Plot of normalized real frequencies corresponding to the growth rate curves in Figure 4.16. The parameter labelling the curves is $T_{i} / T_{e}$ 
(a)

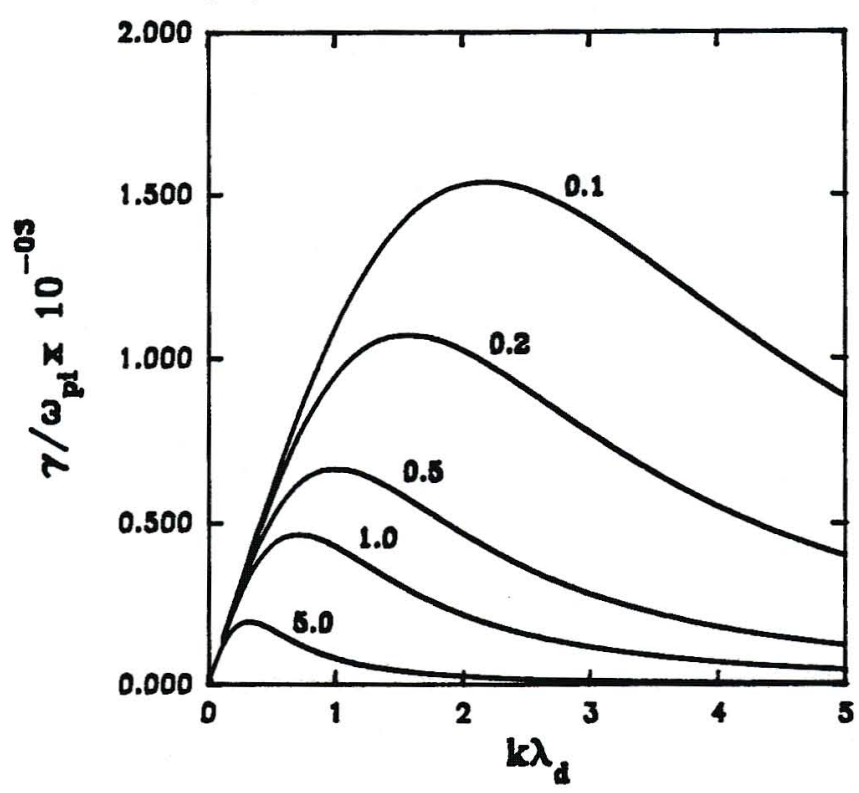

(c)

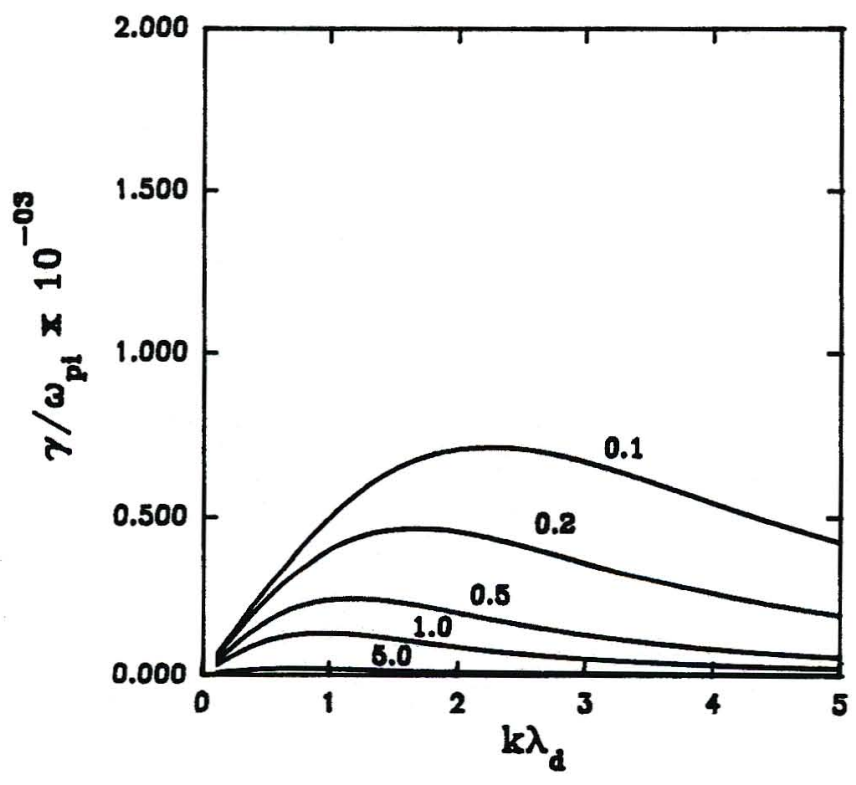

(b)

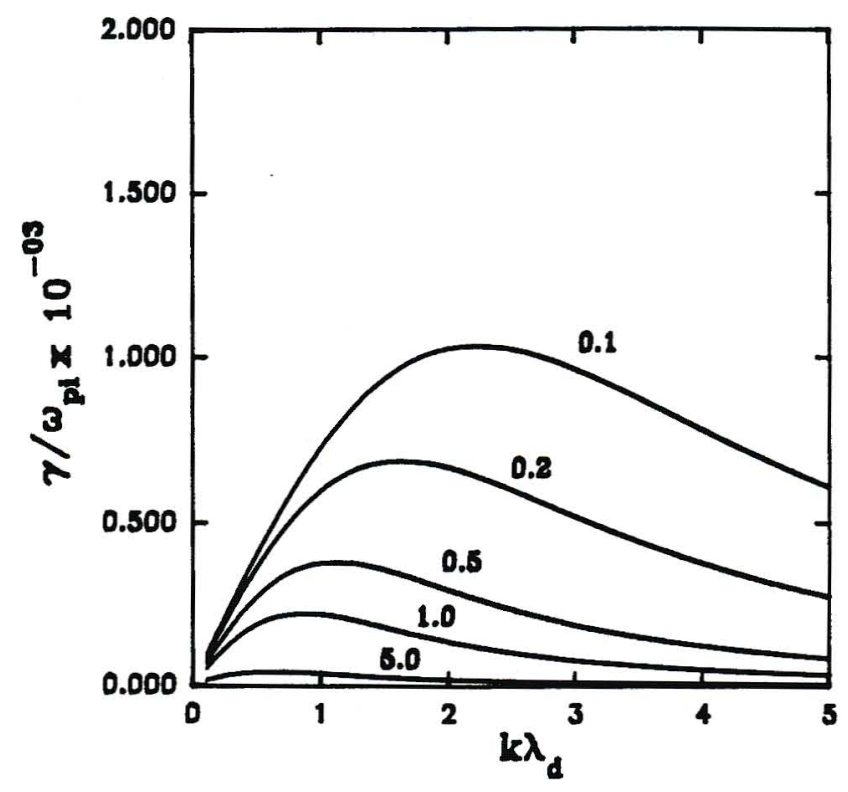

(d)

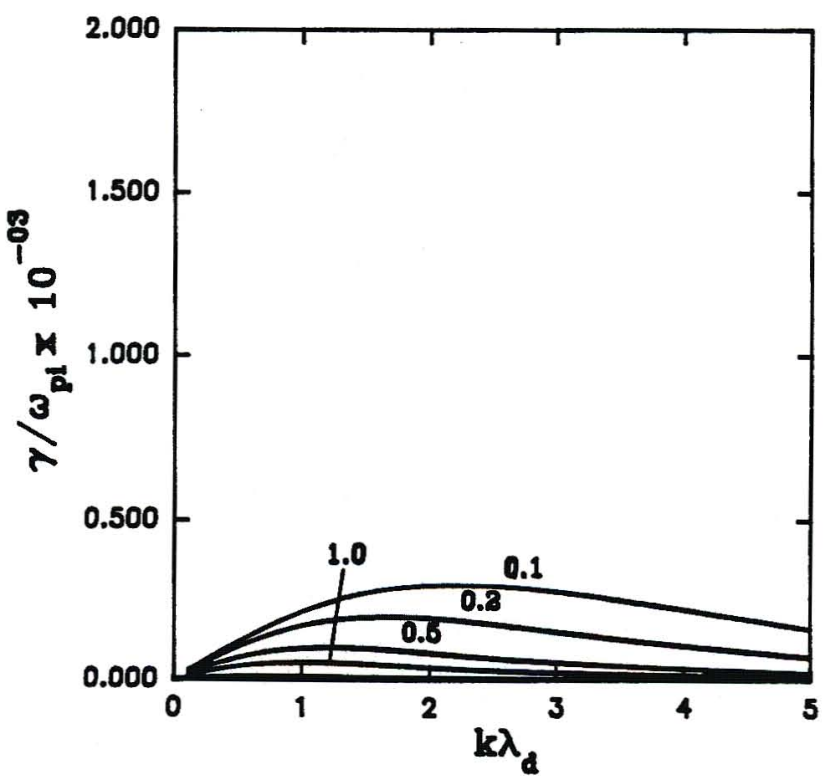

Figure 4.18: Plot of normalized growth rate versus $k \lambda_{d}$ for different values of the equilibrium electron density $n_{e o} / n_{o}$ : (a) $n_{e o}=0.00 n_{o}$, (b) $n_{e o}=0.5 n_{o}$, (c) $n_{e o}=$ $0.75 n_{o}$ and (d) $n_{e o}=0.95 n_{o}$. The parameter labelling the curves is $T_{i} / T_{e}$. All other parameters are as in Figure 4.1 
The effect of the magnetic field strength is depicted in Figure 4.19. As the magnitude of $B_{o}$ increases ( $\omega_{p i} / \Omega_{i}$ decreasing), the range of propagation angles over which the mode is unstable becomes wider and the growth rate increases as for the single heavy dust constituent plasma. Again, for a weakly magnetized plasma (large $\left.\omega_{p i} / \Omega_{i}\right)$, the instability is restricted to a narrow cone of propagation angles about the beam direction. The growth rate is maximum for propagation along $\mathbf{B}_{\mathbf{o}}$ as was the case for the single heavy dust constituent plasma.

We finally investigate the effect of oblique angles of wave propagation relative to $\mathbf{B}_{\mathbf{o}}$. The real frequency (1) and growth rate (2) curves for certain oblique angles of propagation are shown in Figure 4.20 corresponding to $\theta=\sin ^{-1}\left(k_{y} / k\right)=45^{\circ}$ and $30^{\circ}$ respectively. The instability is dust-acoustic in nature. However if we consider a more oblique angle of wave propagation (more perpendicular to $\mathbf{B}_{\mathbf{o}}$ ) such as $k_{y} / k=0.95$ $\left(\theta=72^{\circ}\right)$, then in addition to the dust-acoustic instability, the dust-modified twostream instability (Rosenberg and Krall,1995) is also excited for each value of $\omega_{p i} / \Omega_{i}$ (Figures 4.21-4.23). 


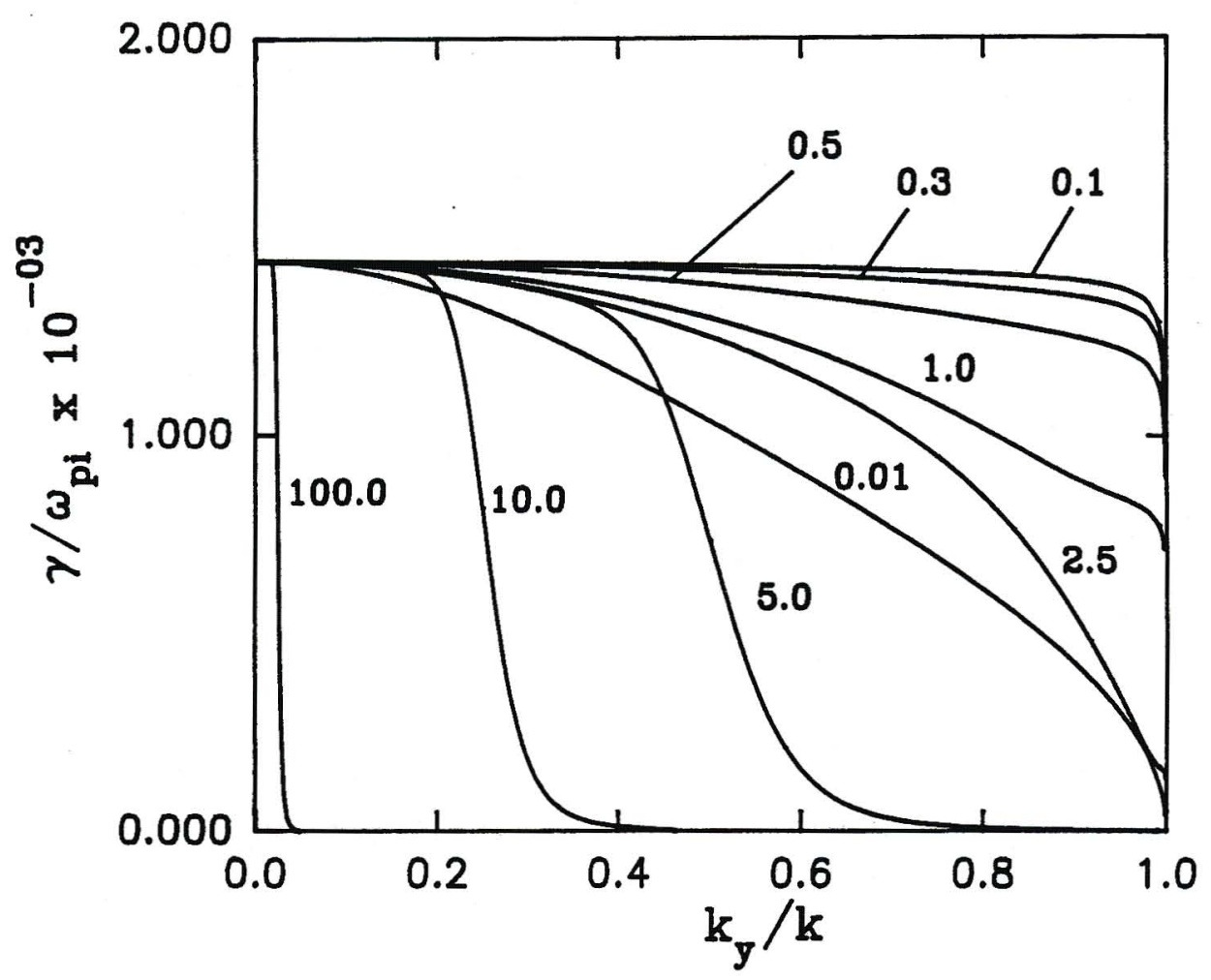

Figure 4.19: Plot of normalized growth rate as a function of $k_{y} / k$. The parameter labelling the curves is $\omega_{p i} / \Omega_{i}$. Fixed parameters are $V_{d i z}=0.2 C_{s}, k \lambda_{d}=2.4$ and $T_{i}=$ $0.1 T_{e}$. All other parameters are as in Figure 4.13 
(1)

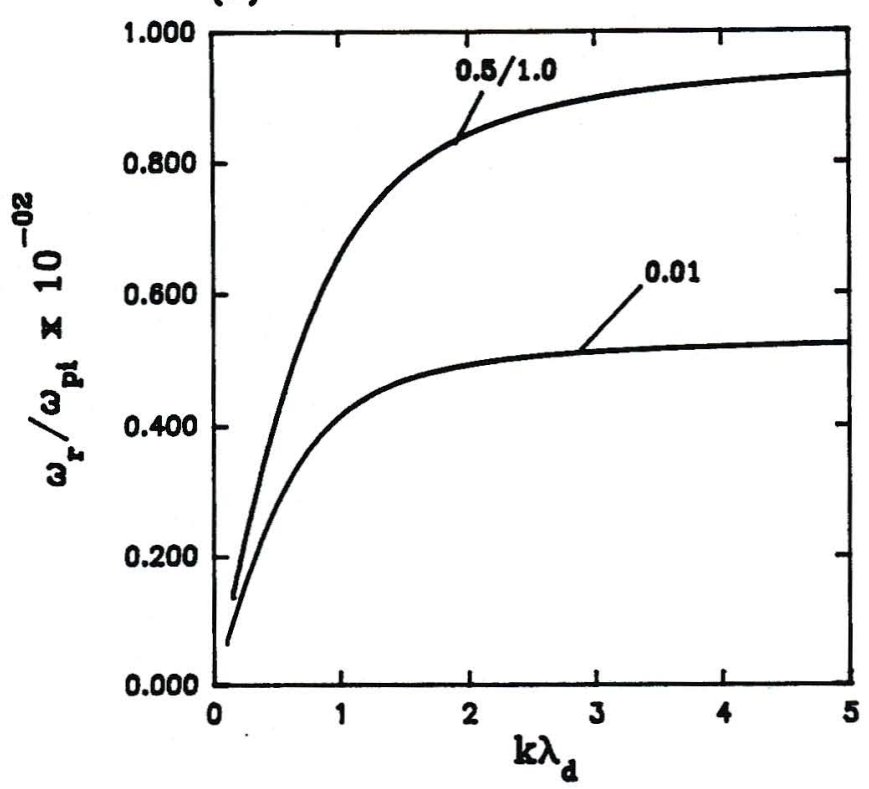

(1)

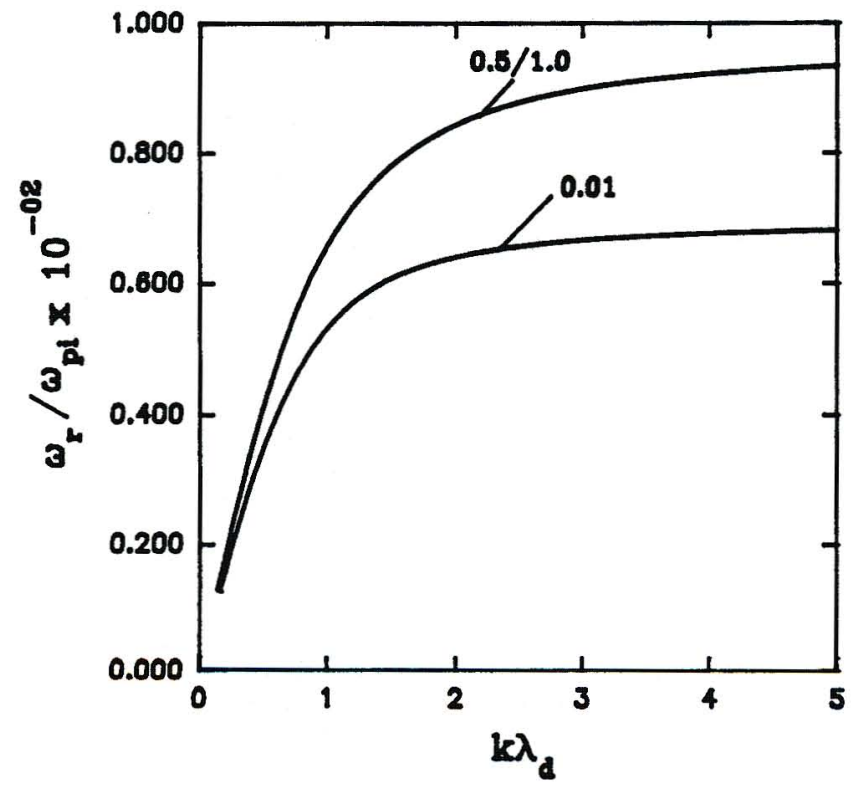

(a)

(2)

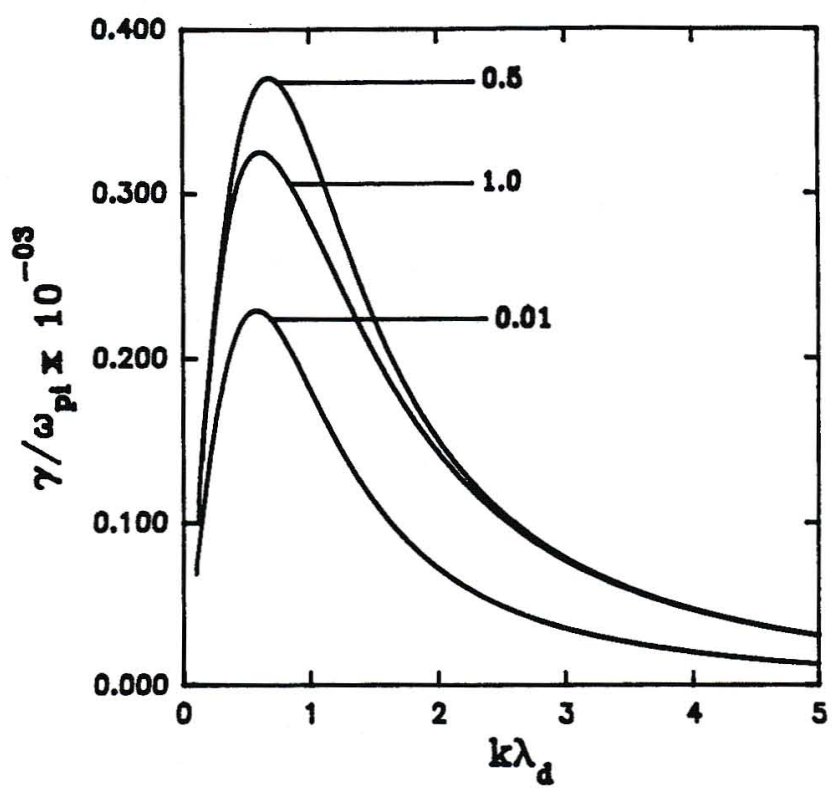

(b)

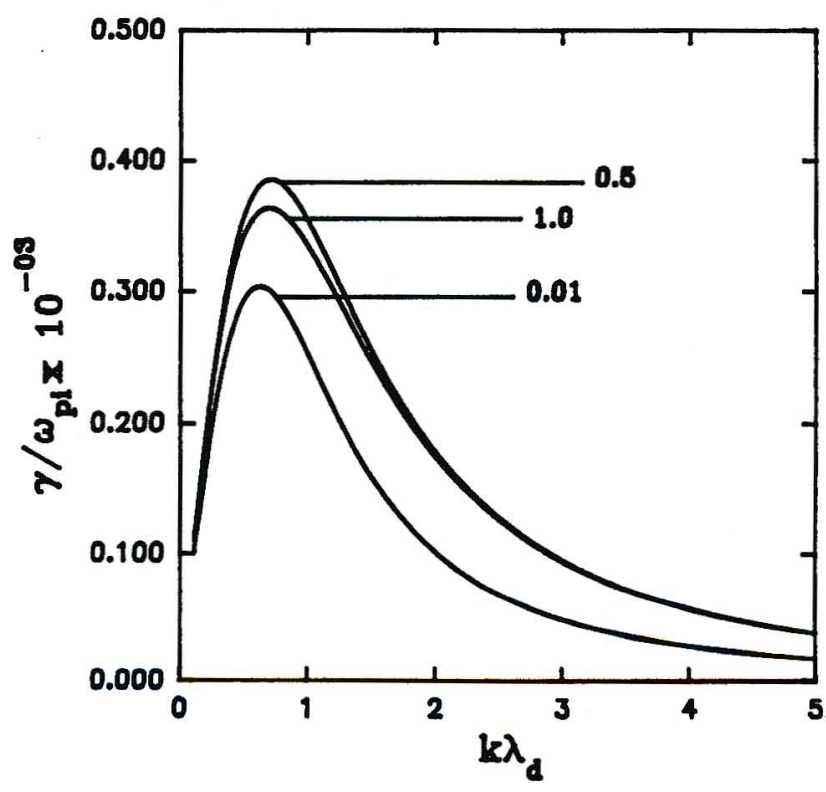

Figure 4.20: Plot of normalized real frequency (1) and growth rate (2) versus $k \lambda_{d}$ for oblique angles of propagation relative to $\mathbf{B}_{\mathbf{o}}$. Here $T_{i}=T_{e}$ for (a) $k_{y} / k=0.707$ $\left(\theta=45^{\circ}\right)$ and (b) $k_{y} / k=0.5\left(\theta=30^{\circ}\right)$. The parameter labelling the curves is $\omega_{p i} / \Omega_{i}$. All other fixed parameters are as in Figure 4.19 
(1)

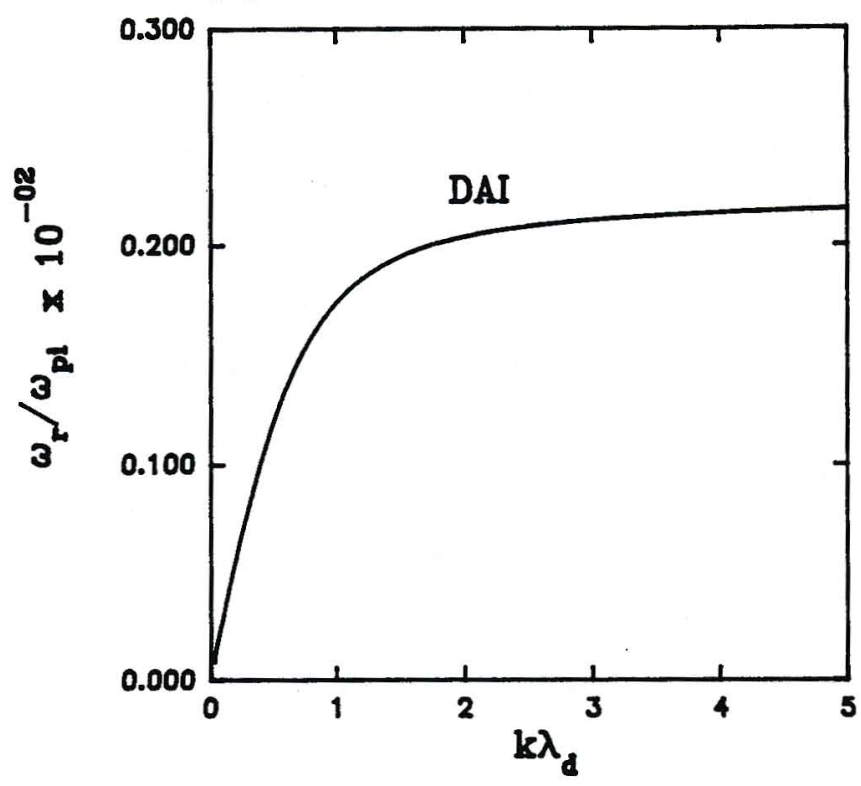

(1)

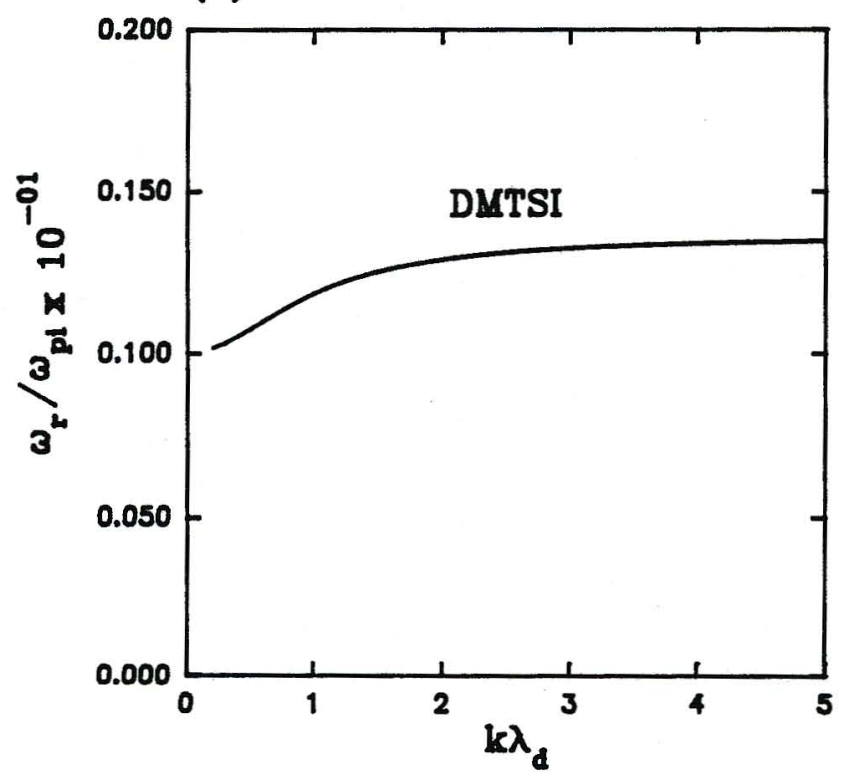

(2)

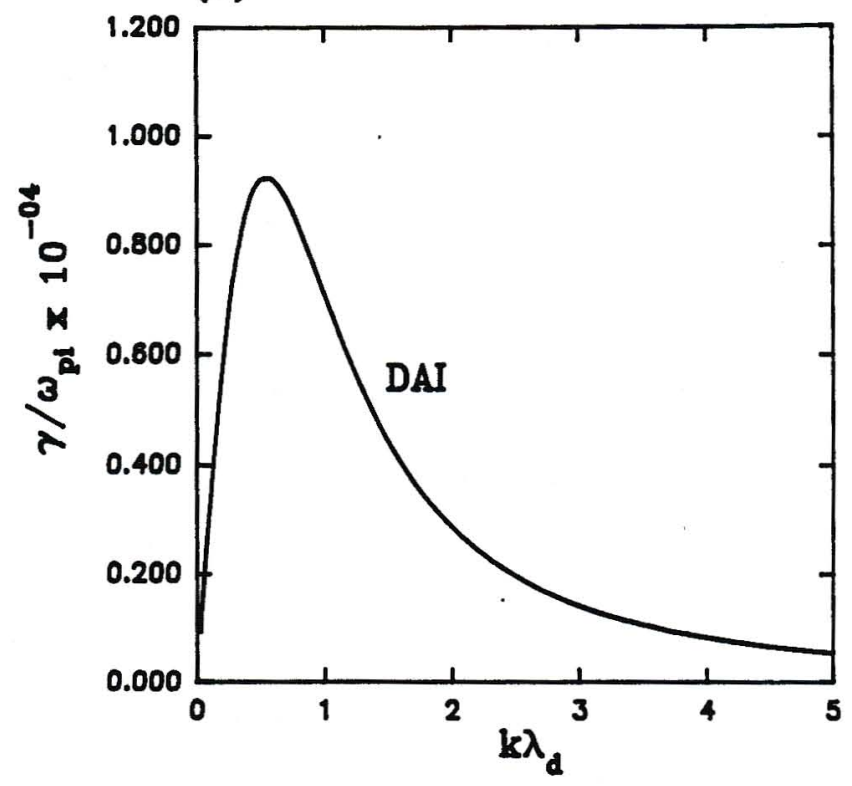

(2)

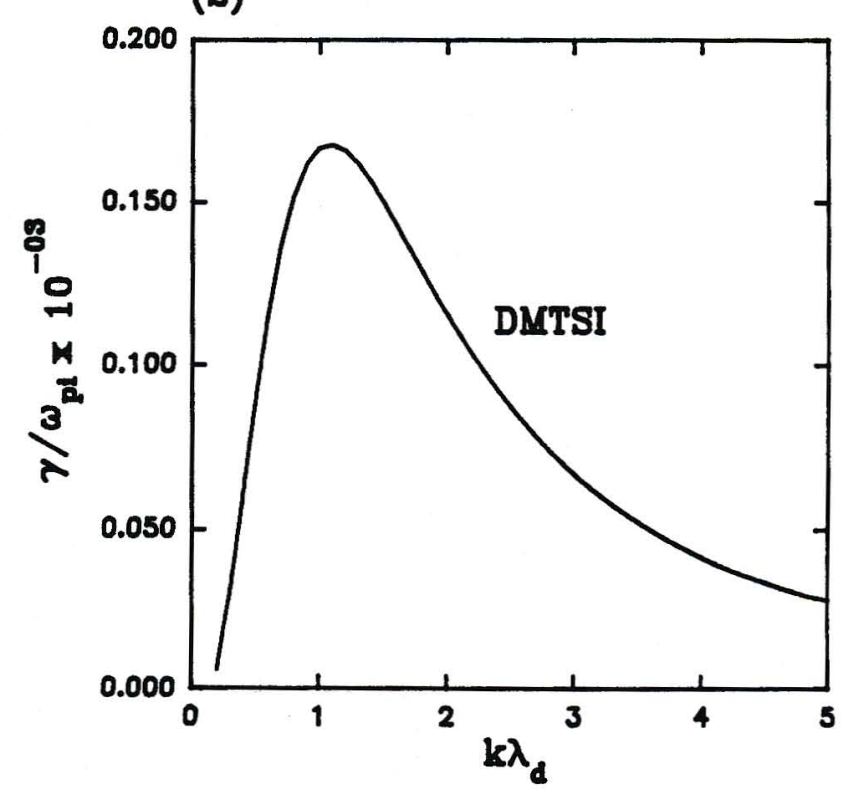

Figure 4.21: Plot of the normalized real frequency (1) and growth rate (2) versus $k \lambda_{d}$ for $k_{y} / k=0.95\left(\theta=72^{\circ}\right)$. Here $\omega_{p i} / \Omega_{i}=0.01$. All other fixed parameters are as in Figure 4.20 
(1)

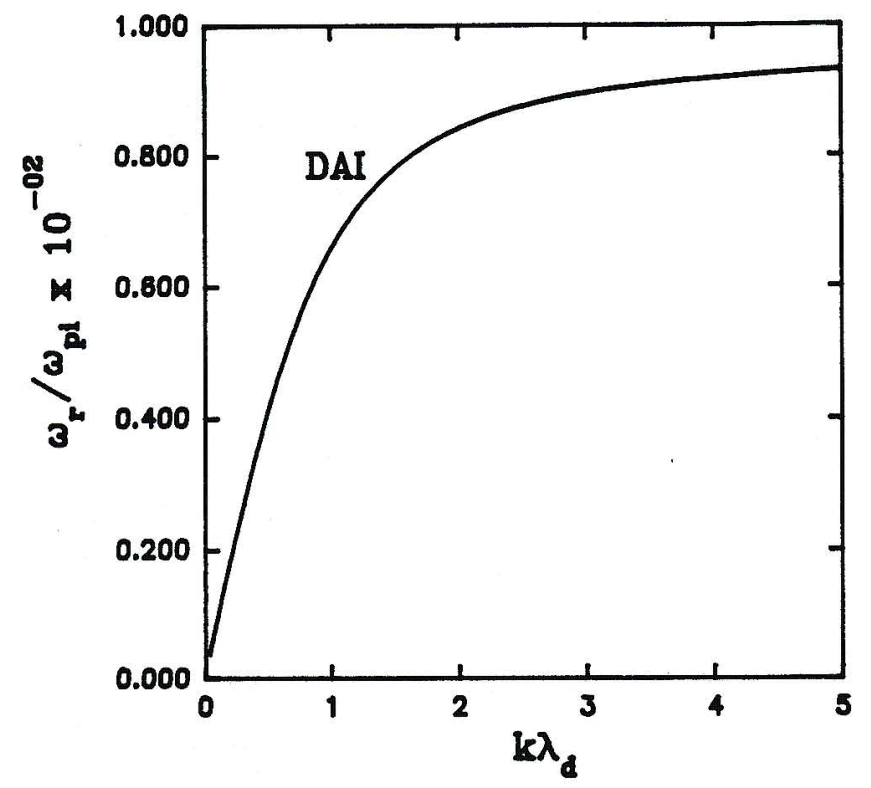

(1)

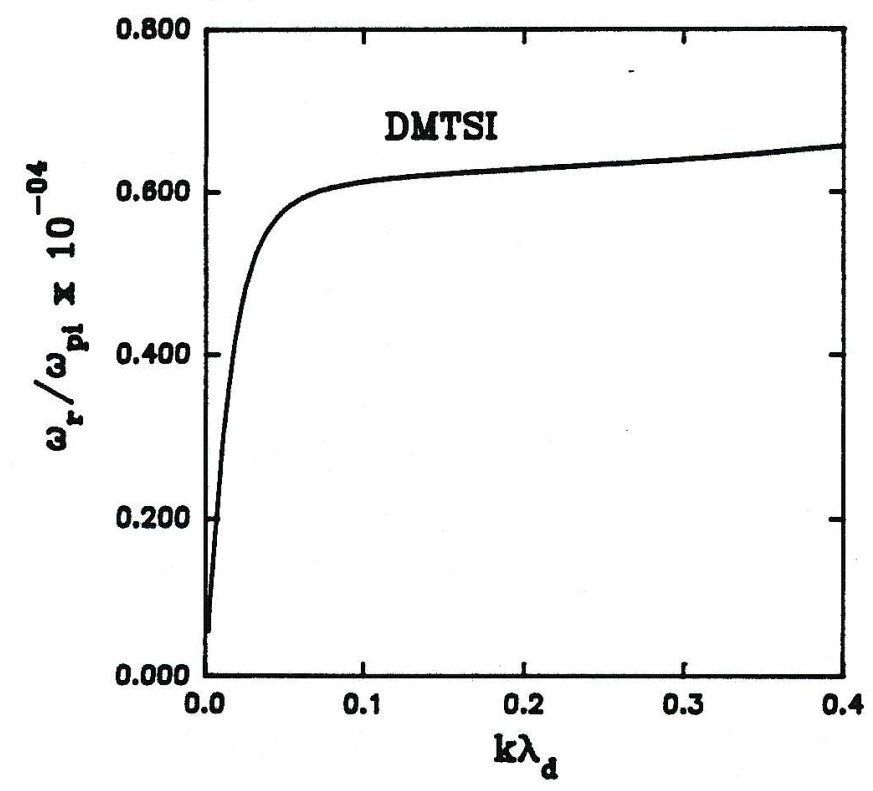

(2)

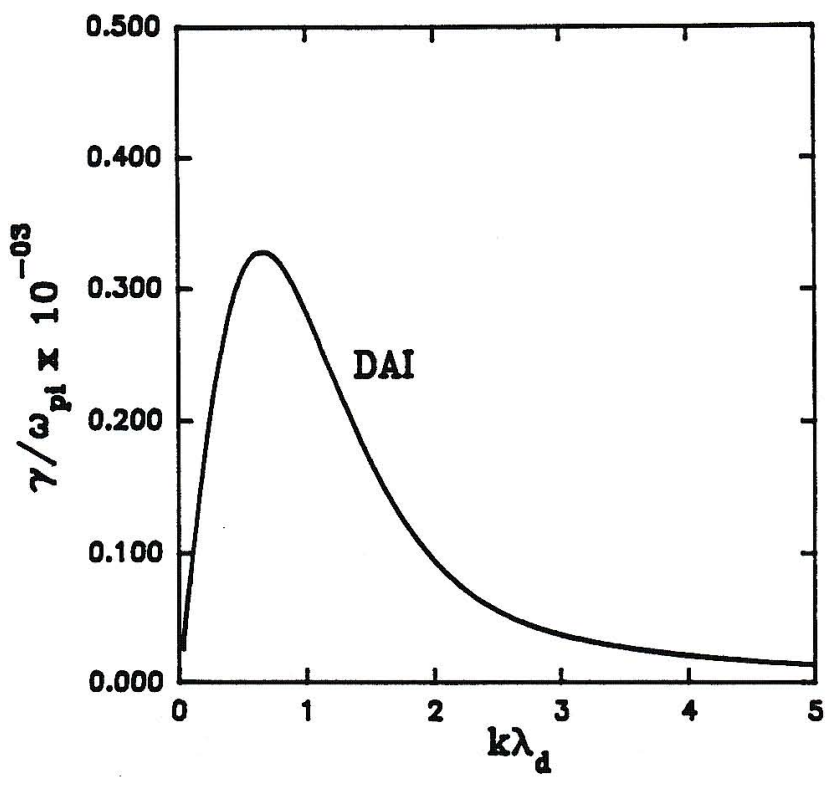

(2)

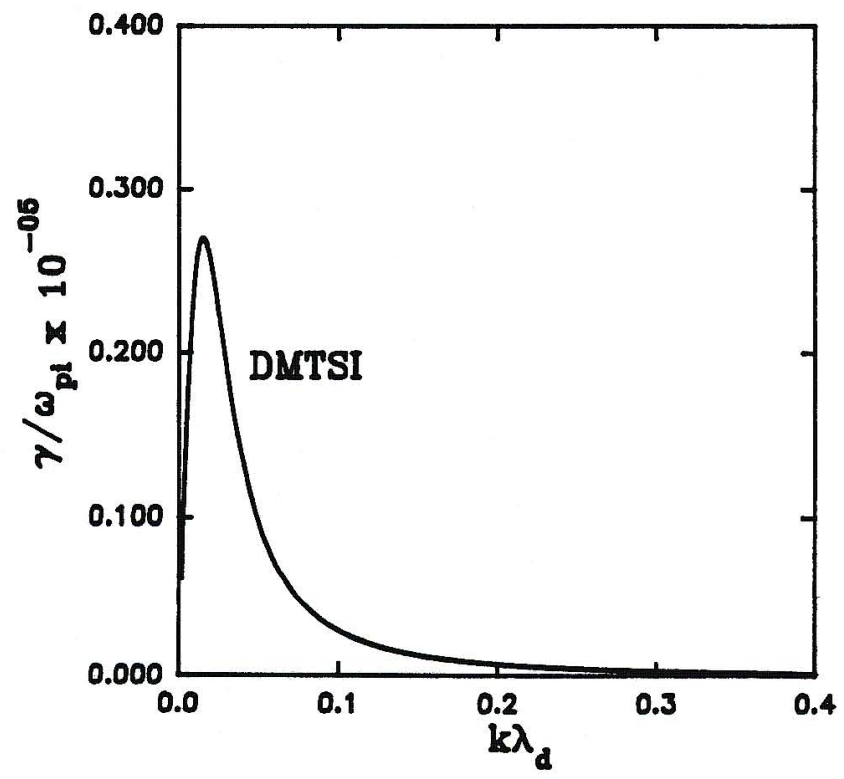

Figure 4.22: Plot of the normalized real frequency (1) and growth rate (2) versus $k \lambda_{d}$ for $k_{y} / k=0.95\left(\theta=72^{\circ}\right)$. Here $\omega_{p i} / \Omega_{i}=0.5$. All other fixed parameters are as in Figure 4.20 
(1)

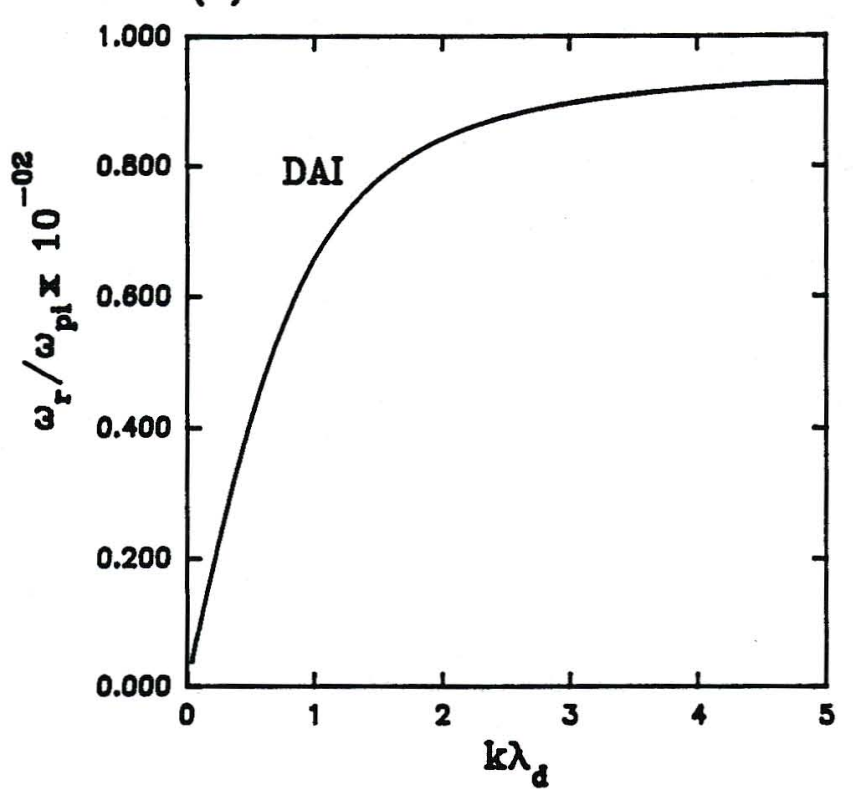

(1)

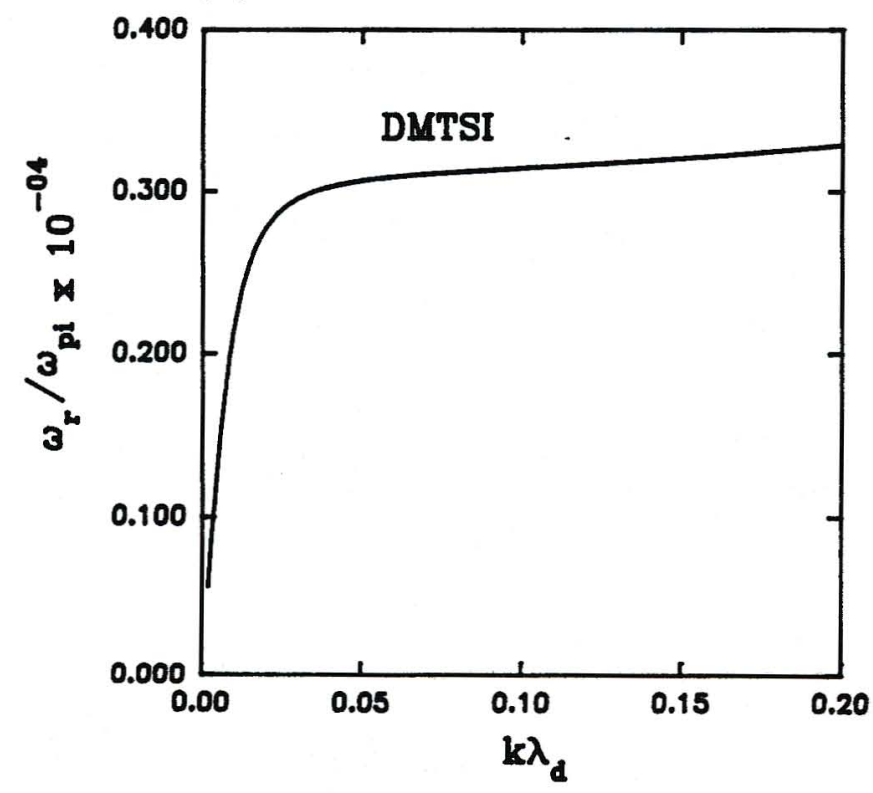

(2)

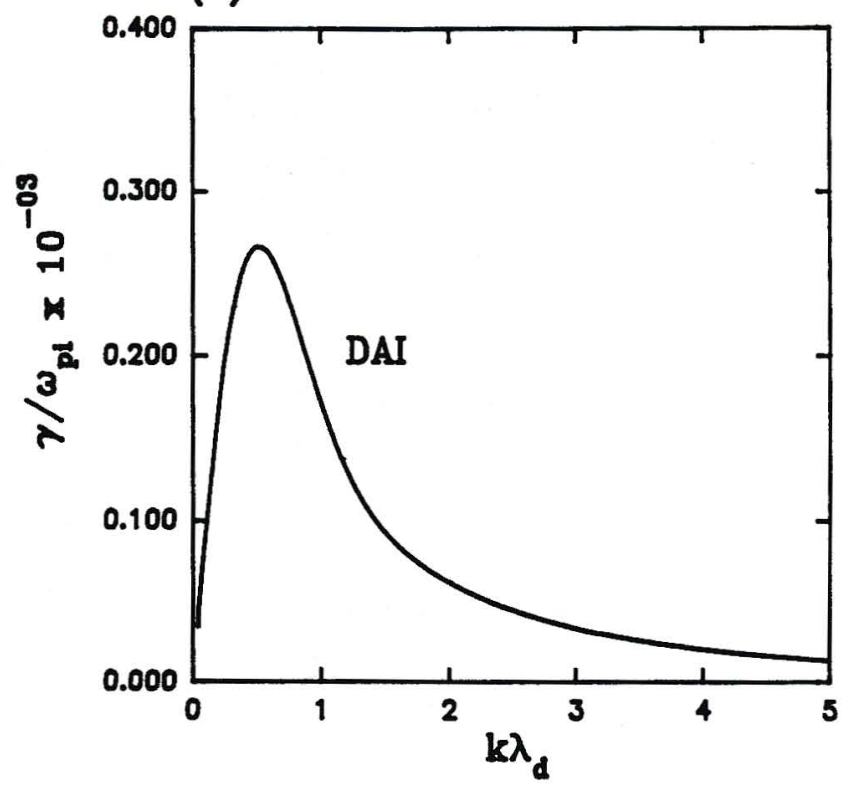

(2)

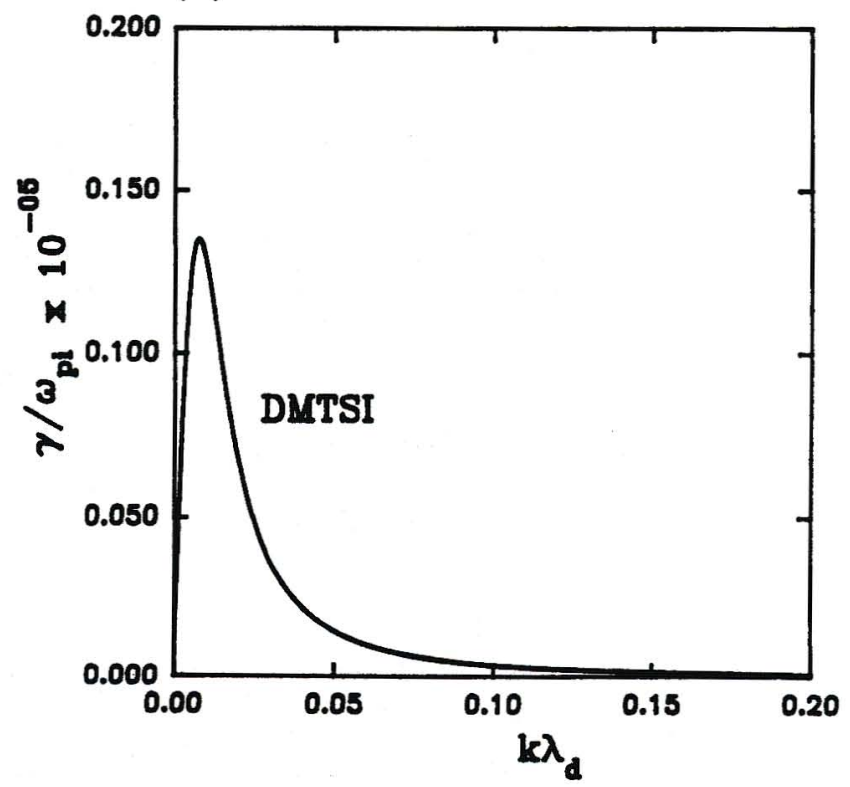

Figure 4.23: Plot of the normalized real frequency (1) and growth rate (2) versus $k \lambda_{d}$ for $k_{y} / k=0.95\left(\theta=72^{\circ}\right)$ and $\omega_{p i} / \Omega_{i}=1.0$. All other fixed parameters are as in Figure 4.20 


\section{Chapter 5}

\section{Solution of the Dispersion \\ Relation for a Four-Component Plasma (Two Dust Species)}

Our analysis is now applied to a four-component plasma comprising of drifting ions, stationary electrons and two stationary dust components (one light and one heavy) where the distinction between light and heavy is due to the different dust grain to ion mass ratios of the two species. All species are considered to be fully magnetized. The velocity distributions of all species are the same as in Chapter 4. Following the procedure outlined in Chapter 4, the general kinetic dispersion relation for the model is obtained to be: 


$$
\begin{aligned}
0=k^{2} & +\frac{1}{\lambda_{d e}^{2}}\left[1+\frac{\omega}{\sqrt{2} k_{z} C_{e}} \sum_{\mathrm{p}=-\infty}^{\infty} Z\left(z_{p e}\right) \Gamma_{p e}\right] \\
& +\frac{1}{\lambda_{d i}^{2}}\left[1+\frac{\omega-\mathbf{k} \cdot \mathbf{V}_{d i}}{\sqrt{2} k_{z} C_{i}} \sum_{\mathrm{p}=-\infty}^{\infty} Z\left(z_{p i}\right) \Gamma_{p i}\right] \\
& +\frac{1}{\lambda_{d d l}^{2}}\left[1+\frac{\omega}{\sqrt{2} k_{z} C_{d l}} \sum_{\mathrm{p}=-\infty}^{\infty} Z\left(z_{p d l}\right) \Gamma_{p d l}\right] \\
& +\frac{1}{\lambda_{d d h}^{2}}\left[1+\frac{\omega}{\sqrt{2} k_{z} C_{d h}} \sum_{\mathrm{p}=-\infty}^{\infty} Z\left(z_{p d h}\right) \Gamma_{p d h}\right]
\end{aligned}
$$

where $e, i, d l$ and $d h$ represent the electron, ion, light and heavy dust components respectively. All symbols have been defined in Chapter 4. This dispersion relation is numerically solved and compared with the approximate analytical solution in the next section.

\subsection{Numerical Results}

We again carry out our analysis in the $y-z$ plane, with $\mathbf{k}=\left(k_{y}, k_{z}\right)=\left(k_{\perp}, k_{z}\right)$. Results are presented in normalized form. Normalizations are the same as in Chapter 4. Quasi-neutrality at equilibrium requires:

$$
n_{e o}+Z_{d l} n_{d l o}+Z_{d h} n_{d h o}=n_{i o}=n_{o}
$$

where $\left(-e Z_{j}\right.$ ) (for $j=d l$ and $d h$ ) is the uniform charge on each dust particle of species $j$.

We initially consider wave propagation along $\mathbf{B}_{o}\left(k_{y} / k=0\right)$. It will later become evident that this will correspond to the direction of propagation for which wave 
growth rate will be a maximum as for the single dust constituent plasmas in the previous chapter. The standard parameter values chosen for the numerical work are $T_{i}=T_{e}, T_{d l}=0.01 T_{e}, T_{d h}=0.01 T_{e}, n_{e o}=0.1 n_{o}, Z_{d l}=10^{2}$ and $Z_{d h}=10^{4}$. The dust grain to ion mass ratios are set to $m_{d l} / m_{i}=10^{6}$ and $m_{d h} / m_{i}=10^{12}$ corresponding to the light and heavy dust constituents respectively. Assuming equal densities of the light and the heavy dust constituents, we set $n_{d l o}=n_{d h o}$. The ion to electron mass ratio is set to $m_{i} / m_{e}=1836$ (corresponding to a hydrogen plasma). In our selection of parameters, we recall (Meuris et al.,1997) that the charge to mass ratio of the dust grains is proportional to the inverse square of the grain radius $\left(q_{d} / m_{d} \sim a^{-2}\right)$. We again consider ions and dust particles which are weakly magnetized $\left(\omega_{p i} / \Omega_{i}=100\right)$. For these fixed parameters, $\omega_{p i} / \omega_{p d l}=1.1 \times 10^{2}$ and $\omega_{p i} / \omega_{p d h}=1.1 \times 10^{4}$ corresponding to the light and heavy dust species respectively.

Figure 5.1 shows that as $V_{d i z} / C_{s}$ increases, there is an increase in the growth rate of the instability due to more free energy becoming available to drive the instability. We notice that the range of wave numbers for which the dust-acoustic mode is unstable, is reduced when compared to the case of the single dust constituent plasmas of the previous chapter. In Figure 5.1 we see that for fixed $V_{d i z}=0.5 C_{s}$ the mode is damped beyond $k \lambda_{d} \approx 3.0$, whereas in the case of the single heavy dust constituent plasma (Figure 4.1), the upper limit of normalized wave numbers for which the mode is unstable is approximately 5.0. As was observed by Bharuthram et al. (1996) for a three-component plasma, there is no growth for $V_{d i z}<0.01 C_{s}$ because the beam is not energetic enough to drive the instability. 


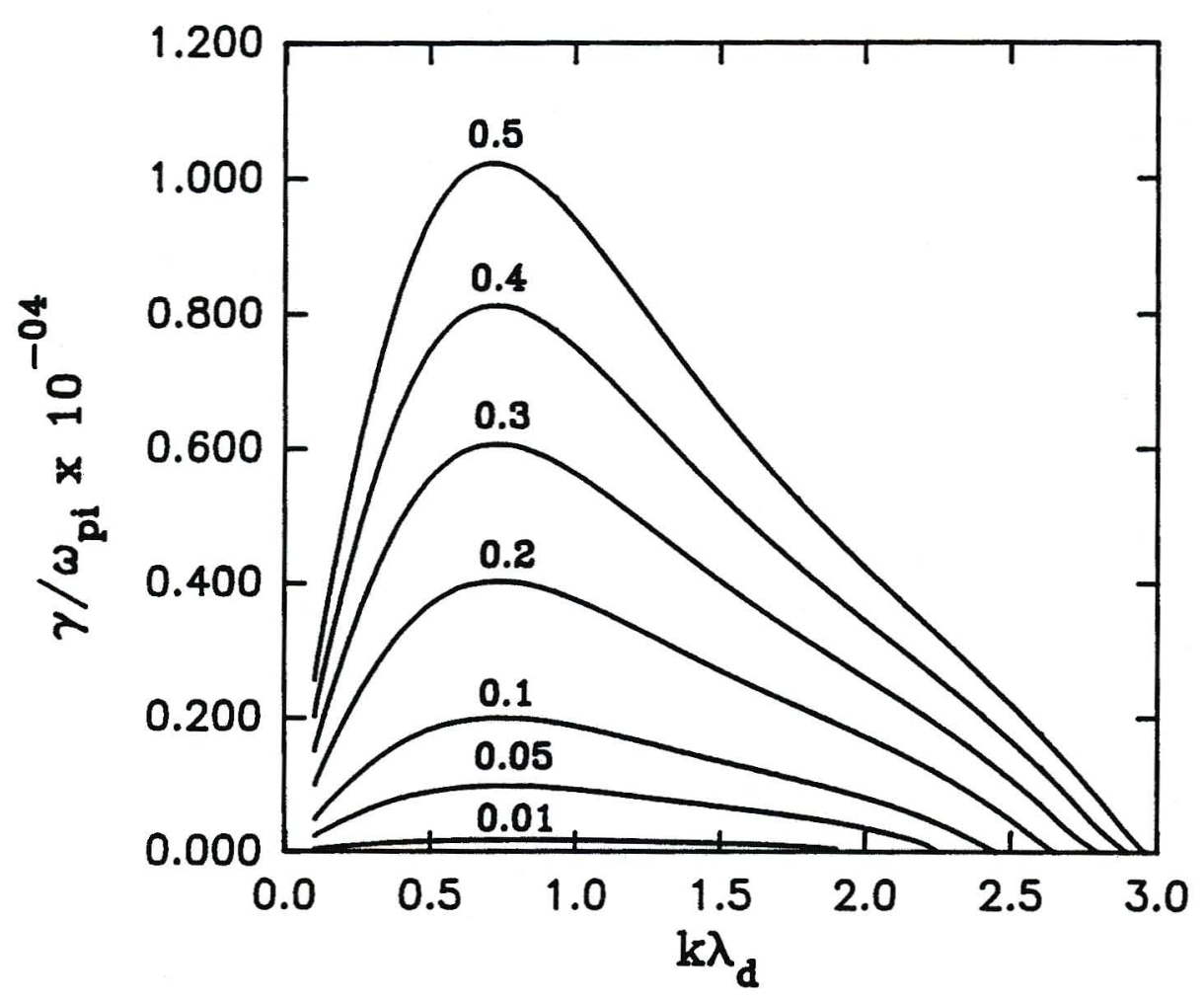

Figure 5.1: Plot of normalized growth rate versus normalized wavenumber. The parameter labelling curves is the normalized ion drift speed $V_{d i z} / C_{s}$. The fixed parameters are $T_{i}=T_{e}, T_{d l}=0.01 T_{e}, T_{d h}=0.01 T_{e}, n_{e o}=0.1 n_{o}, n_{d l o}=n_{d h o}, m_{d l} / m_{i}=$ $10^{6}, Z_{d l}=10^{2}, m_{d h} / m_{i}=10^{12}$ and $Z_{d h}=10^{4}$ 
The real frequency curves corresponding to the growth rate curves of Figure 5.1 are not shown because the real frequency is numerically found to be independant of ion drift velocity. This is confirmed by analytically solving for the real frequency for a four-component (two dust species) plasma by making appropriate expansions of the Z function (Bharuthram et al.,1996). We find

$$
\omega_{r}^{2}=k_{z}^{2} C_{s}^{2} \frac{\left\{\frac{m_{i}}{m_{d l}} Z_{d l}\left(\delta-1-Z_{d h} \frac{n_{d h o}}{n_{e o}}\right)+\frac{m_{i}}{m_{d h}} Z_{d h}\left(\delta-1-Z_{d l} \frac{n_{d l o}}{n_{e o}}\right)\right\}}{\left(1+\delta T_{e} / T_{i}+\delta k^{2} \lambda_{d}^{2}\right)}
$$

where $\delta=\left(n_{i o} / n_{e o}=n_{o} / n_{e o}\right)$. We recall that the subscripts $d l$ and $d h$ refer to the light and heavy dust constituents respectively. In arriving at (5.3) we have used the approximations $|\omega| \ll \Omega_{i}$ and $T_{e} \approx T_{i} \gg T_{d h}, T_{d l}$. By setting the number density of either one of the dust species equal to zero $\left(n_{d h o}=0\right.$ or $\left.n_{d l o}=0\right)$, the equation (5.3) reduces to the approximate expression of the real frequency for a three-component (single dust constituent) plasma derived by Rosenberg (1993) for the dust-acoustic mode in the limit $k_{y} / k=0$.

In Figure 5.2 the numerical solution of the real frequency of the full dispersion relation is compared with the approximate analytical solution (5.3). We see that the upper limit of normalized wave numbers $k \lambda_{d}$ for which we get excellent agreement is approximately 0.5 , beyond which there is a deviation of the analytical from the numerical result. 
The analytical expression of the growth rate derived for a four-component plasma (two dust species) is

$$
\begin{gathered}
\gamma \approx \sqrt{\frac{\pi}{8}}\left\{\frac{C_{s}^{2}\left[\frac{m_{i}}{m_{d h}} Z_{d h}\left(\delta-1-Z_{d l} \frac{n_{d l o}}{n_{e o}}\right)+\frac{m_{i}}{m_{d l}} Z_{d l}\left(\delta-1-Z_{d h} \frac{n_{d h o}}{n_{e o}}\right)\right]^{3 / 2} \omega_{r}}{\left(1+\delta T_{e} / T_{i}+\delta k^{2} \lambda_{d}^{2}\right)^{3 / 2}\left\{\frac{T_{e}}{m_{d h}} Z_{d h}\left(\delta-1-Z_{d l} \frac{n_{d l o}}{n_{e o}}\right)+\frac{T_{e}}{m_{d l}} Z_{d l}\left(\delta-1-Z_{d h} \frac{n_{d h o}}{n_{e o}}\right)\right\}}\right\} \\
\times\left[-\left(\frac{m_{e}}{m_{i}}\right)^{1 / 2} \Gamma_{o e}+\delta\left(\frac{T_{e}}{T_{i}}\right)^{3 / 2}\left(\frac{\vec{k} \cdot \vec{V}_{o}}{\omega_{r}}-1\right)\right. \\
-\left(\frac{T_{e}}{T_{d h}}\right)^{3 / 2}\left(\frac{m_{d h}}{m_{i}}\right)^{1 / 2} Z_{d h}\left(\delta-1-Z_{d l} \frac{n_{d l o}}{n_{e o}}\right) e^{-z_{o d h}{ }^{2}} \\
\left.-\left(\frac{T_{e}}{T_{d l}}\right)^{3 / 2}\left(\frac{m_{d l}}{m_{i}}\right)^{1 / 2} Z_{d l}\left(\delta-1-Z_{d h} \frac{n_{d h o}}{n_{e o}}\right) e^{-z_{o d l}{ }^{2}}\right]
\end{gathered}
$$

where

$$
\begin{aligned}
z_{o j}{ }^{2}= & \frac{T_{e}}{2 T_{j}} \frac{m_{j}}{m_{i}}\left\{\frac{m_{i}}{m_{d h}} Z_{d h}\left(\delta-1-Z_{d l} \frac{n_{d l o}}{n_{e o}}\right)\right. \\
& \left.+\frac{m_{i}}{m_{d l}} Z_{d l}\left(\delta-1-Z_{d h} \frac{n_{d h o}}{n_{e o}}\right)\right\} \frac{1}{\left(1+\delta \frac{T_{e}}{T_{i}}+\delta k^{2} \lambda_{d}^{2}\right)}
\end{aligned}
$$

for $j=d h$ and $d l$ for the heavy and light dust components respectively.

In Figure 5.3 we see the comparison between the numerical and the approximate analytical solution of the growth rate. Numerically we see that the upper limit of normalized wave numbers for which there is growth $(\gamma>0)$ is approximately 2.75 , beyond which the Landau damping due to the negatively charged components becomes significant and the mode is damped. Analytical considerations of the full dispersion relation predict that the dust-acoustic mode is unstable for a slightly wider range of wave numbers, the upper limit of normalized wave numbers in this case being $k \lambda_{d} \approx 4.0$. 


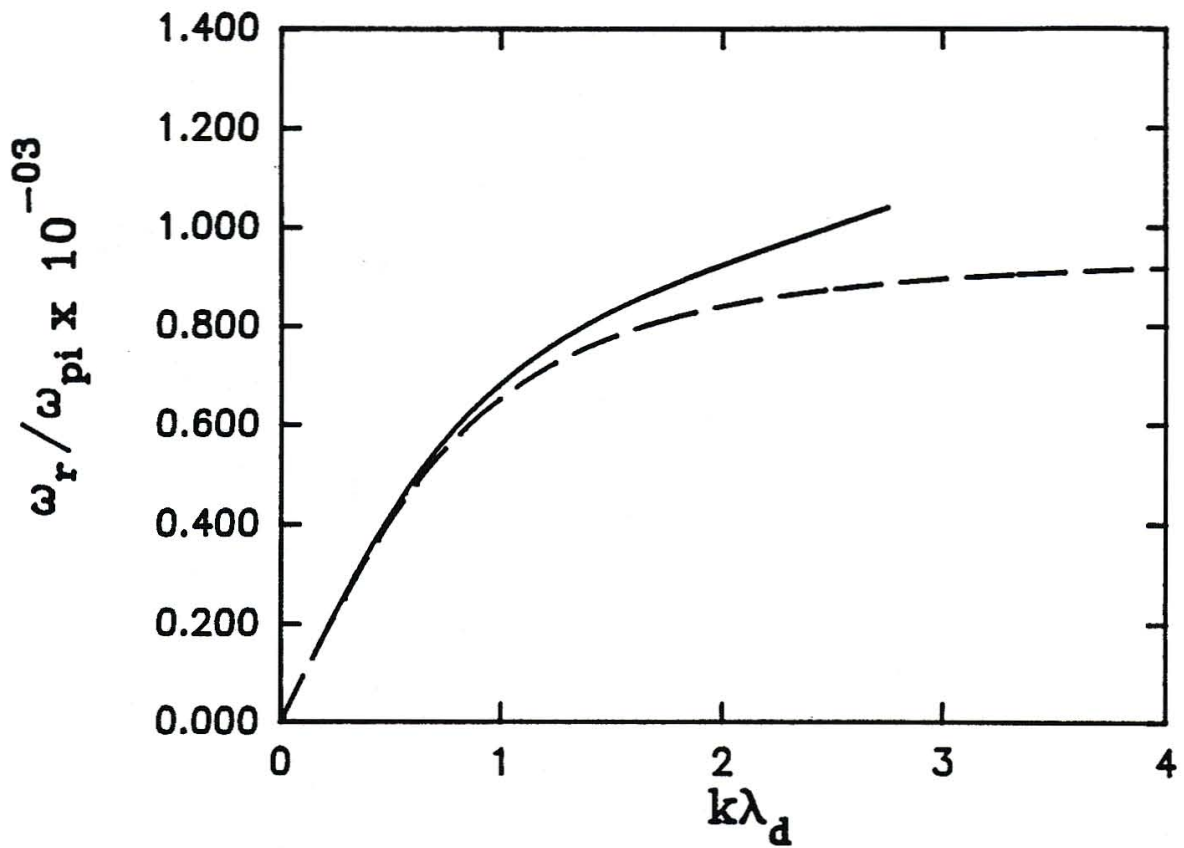

Figure 5.2: Plot of the normalized real frequency against normalized wavenumber for $V_{d i z}=0.3 C_{s}$. The dashed curve shows the result using the approximate analytic expression (5.3). All other fixed parameters are as in Figure 5.1

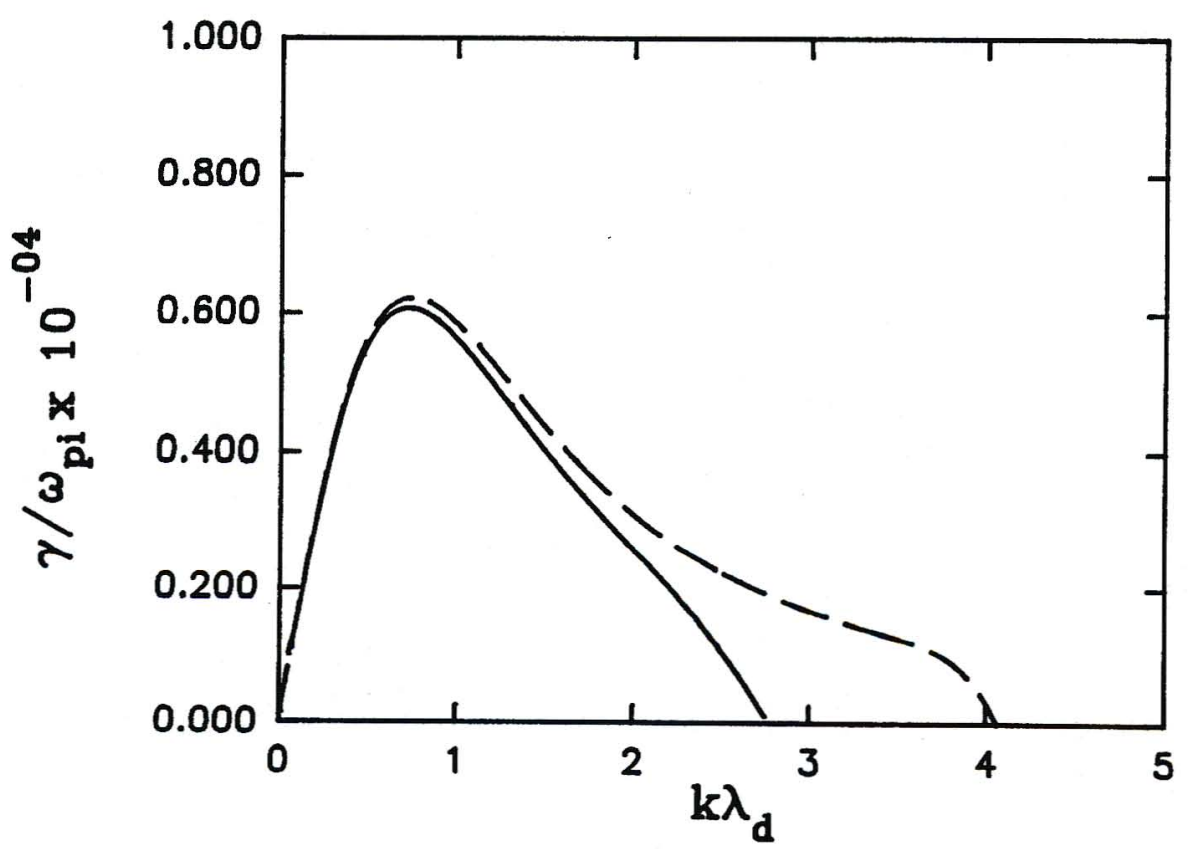

Figure 5.3: Plot of the normalized growth rate as a function of normalized wave number for $V_{d i z}=0.3 C_{s}$. The dashed curve shows the result calculated from the approximate expression (5.4). All other parameters are as in Figure 5.1 
Figure 5.4 shows the effect of varying the concentration of the light relative to the heavy dust grains (in the form of the density ratio $n_{d l o} / n_{d h o}$ ) on the real frequency of the instability. The flat 'plateau' regions labelled $\mathrm{H}$ and $\mathrm{L}$ correspond to the real frequency of the dust-acoustic instability of the single dust constituent plasmas (three-component) of the heavy and light dust species respectively. The transition from the one mode to the other is continuous and occurs over an extremely large range of $n_{d l o} / n_{d h o}$. For a typical value of equal densities of the heavy and light dust constituents $\left(n_{d l o}=n_{d h o}\right)$, the real frequency lies intermediate to those corresponding to the light and heavy dust species of the single dust constituent (three-component) plasmas of Bharuthram et al. (1996). This behaviour is in agreement with Figure 5.6 which shows the approximate solutions of real frequency for the three-component plasmas corresponding to the light and heavy dust constituents and a four-component (two dust species) plasma assuming equal concentrations of the light and the heavy dust species. Figure 5.5 shows the numerical growth rate corresponding to the real frequency curve of Figure 5.4. We observe that for $n_{d l o} / n_{d h o} \leq 10^{-3}\left(n_{d l o} / n_{d h o} \geq 10^{3}\right)$ the heavy (light) dust-acoustic real frequency and growth rate are independant of the light (heavy) dust species. 


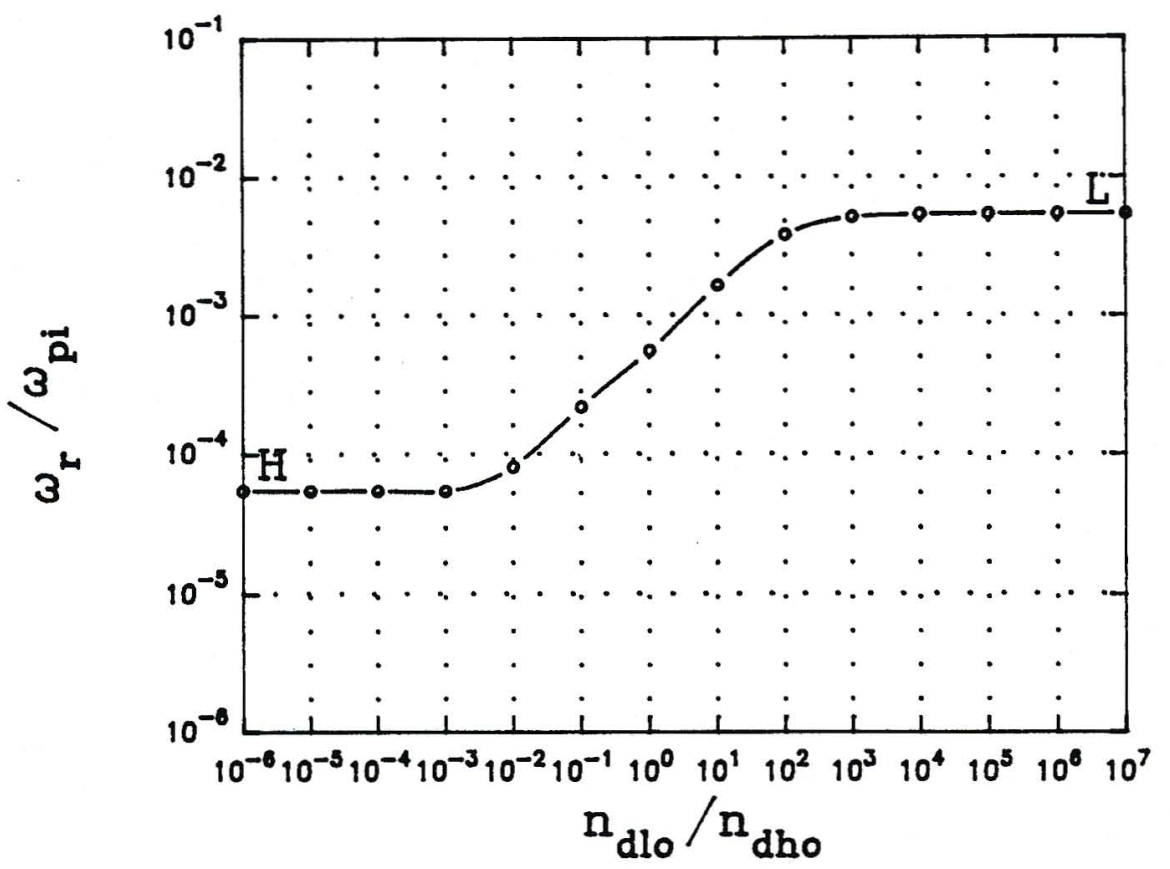

Figure 5.4: Plot of the normalized real frequency versus $n_{d l o} / n_{d h o}$ for $V_{d i z}=0.2 C_{s}$. Here $k \lambda_{d}=0.7$. All other fixed parameters are as in Figure 5.1

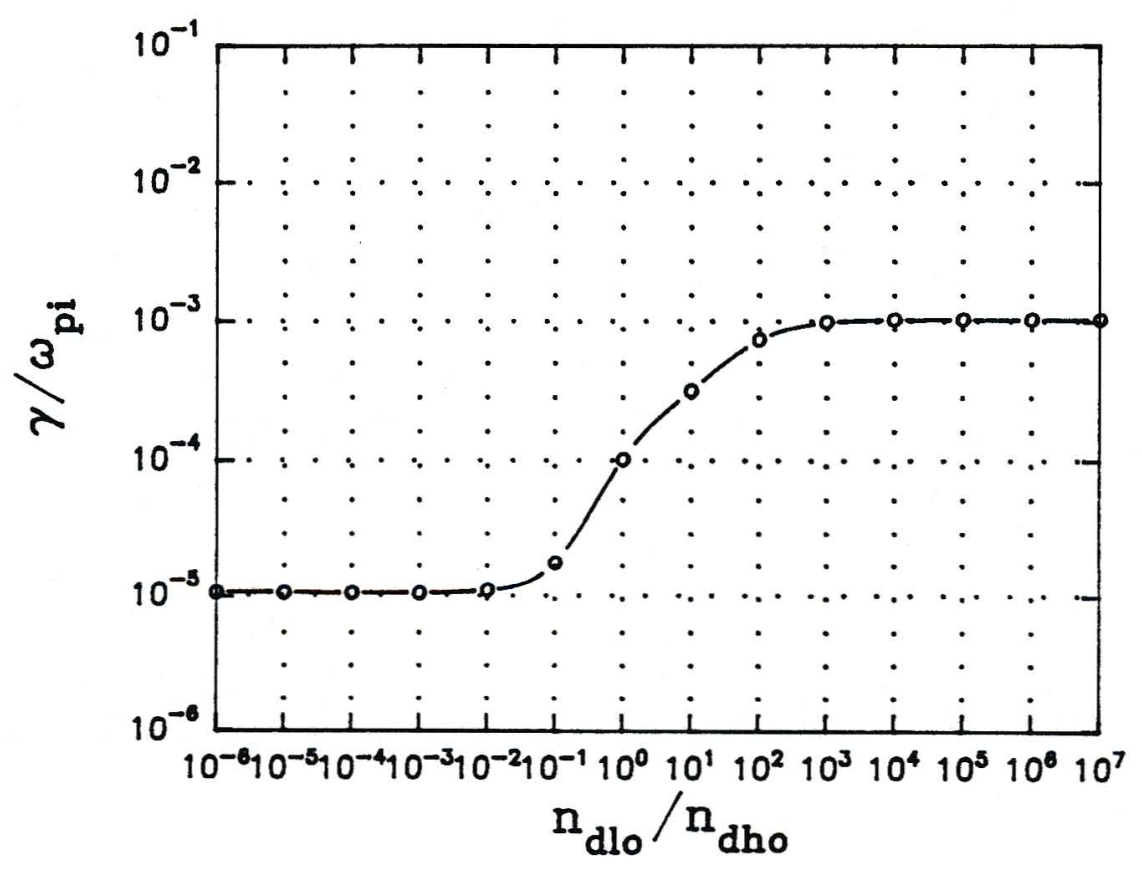

Figure 5.5: Plot of the normalized growth rate corresponding to the real frequency curve of Figure 5.4 


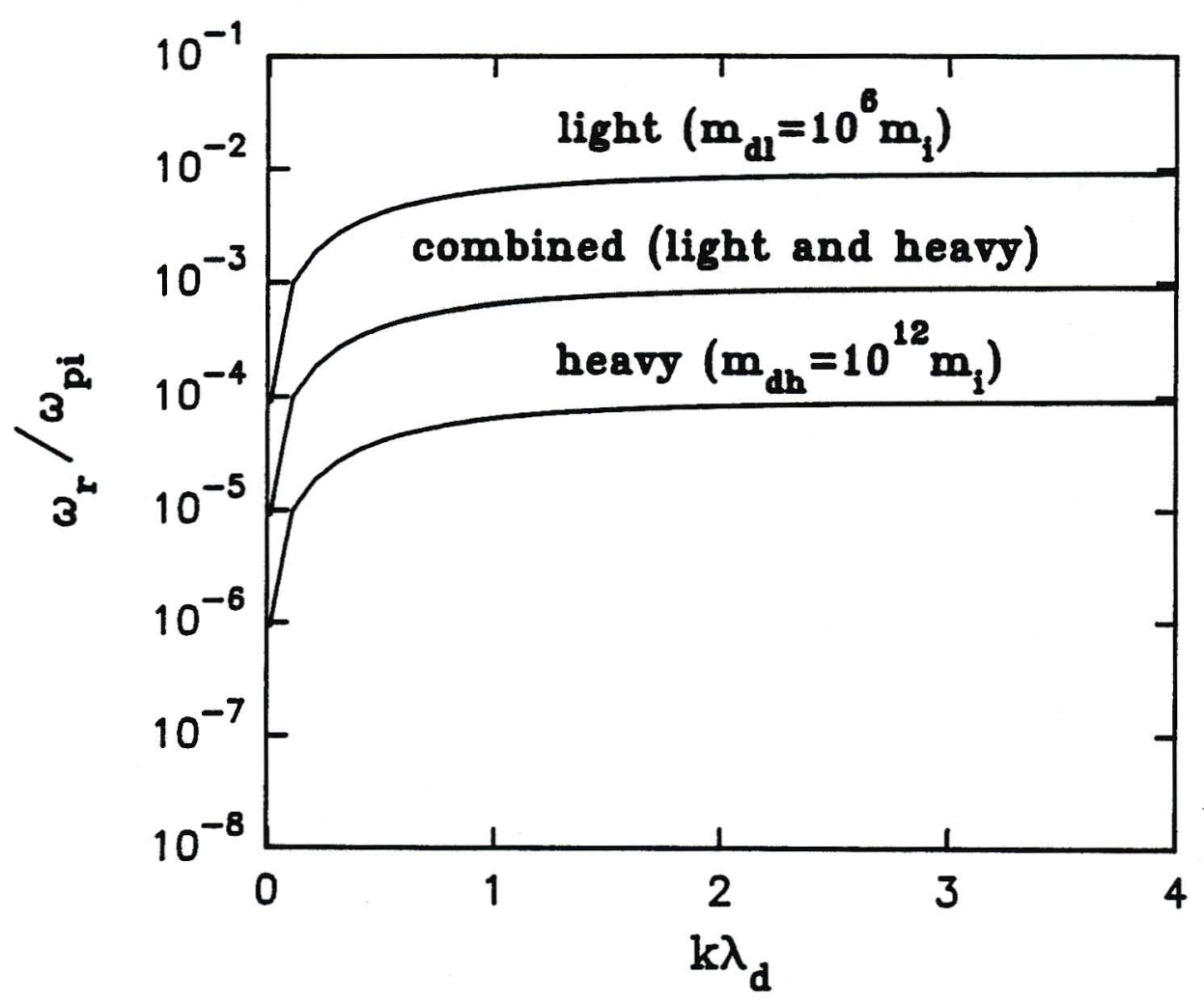

Figure 5.6: Plot of the analytical solutions of real frequency versus normalized wave number for the three-component plasmas corresponding to the light and heavy dust constituents and for a four-component (combined light and heavy dust species) plasma. All fixed parameters are as in Figure 5.1 
The effect of the variation of electron density on the growth rate of the instability is shown in Figure 5.7. As the equilibrium electron density decreases, the growth rate increases which is consistent with the findings in the previous chapter. The range of normalized wave numbers over which the mode is unstable narrows as $n_{e o} / n_{\circ}$ increases. It is evident from Figure 5.7 that as $n_{e o} / n_{o}$ increases, the curves peak at smaller values of $k \lambda_{d}$. For the three-component plasmas in the previous chapter, the curves peak at the same value of $k \lambda_{d} \approx 0.75$ (Figure 4.4). The real frequencies corresponding to the growth rate curves of Figure 5.7 are shown in Figure 5.8. 


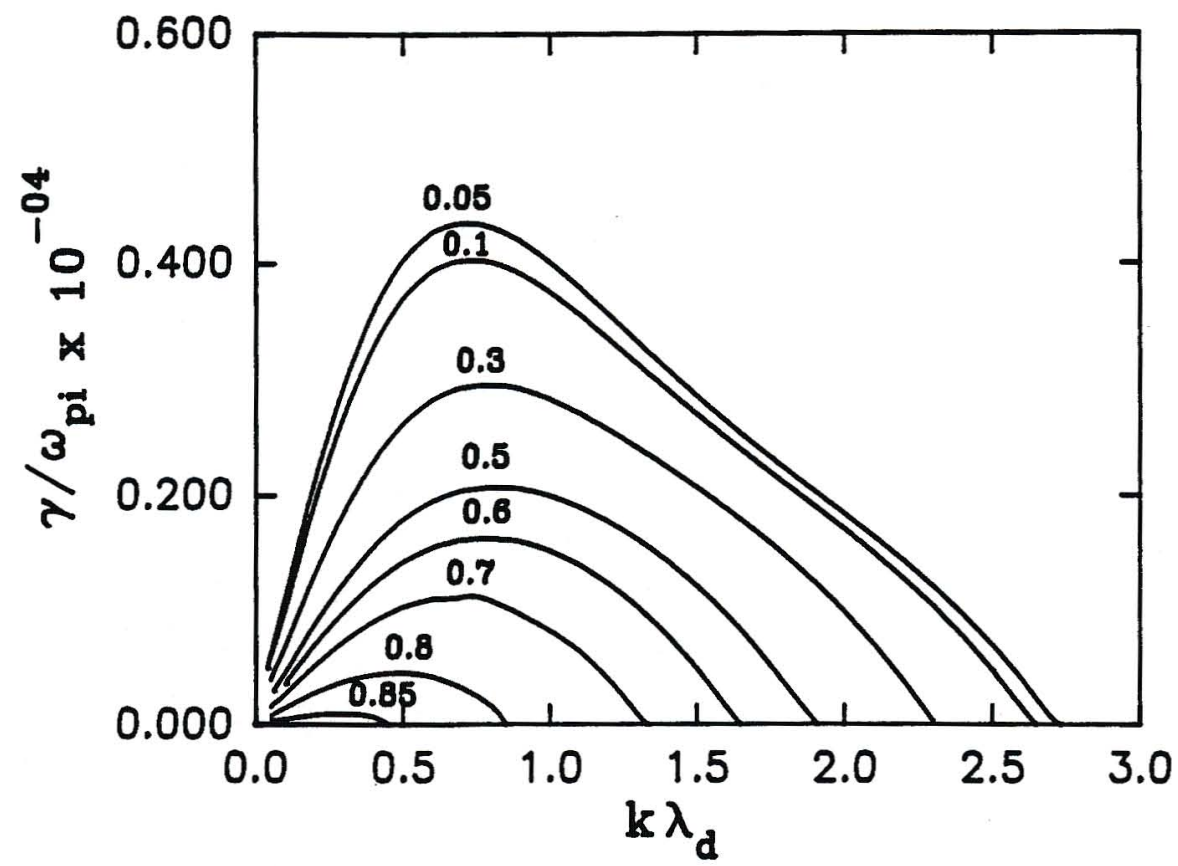

Figure 5.7: Plot of the normalized growth rate versus normalized wave number for $V_{d i z}=0.2 C_{s}$. The parameter labelling the curves is the normalized electron density $n_{e o} / n_{o}$. All other fixed parameters are as in Figure 5.1

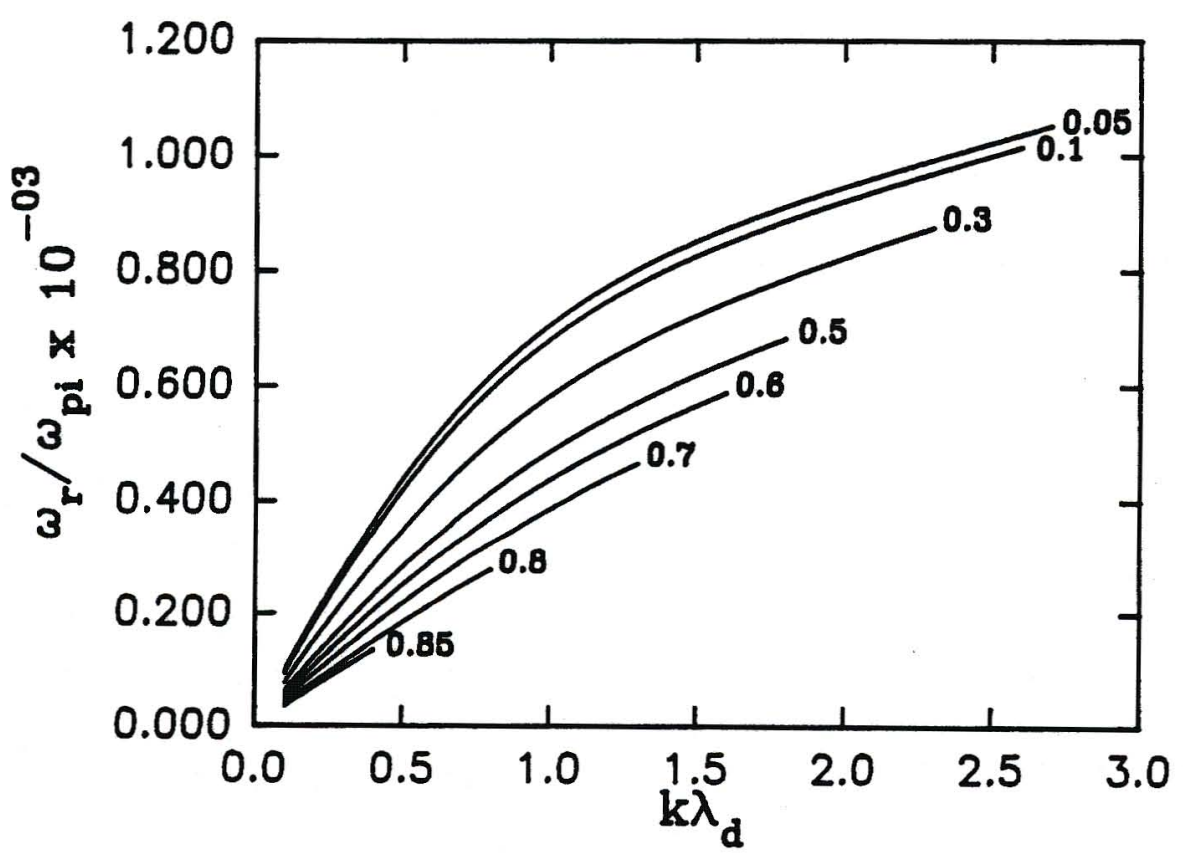

Figure 5.8: Plot of normalized real frequencies corresponding to the growth rates in Figure 5.7. The parameter labelling the curves is $n_{e o} / n_{o}$ 
Figure 5.9 demonstrates that the effect of increasing the equilibrium electron density is that the Landau damping becomes more significant. For nonzero electron densities (Figures 5.9(b) and (c)), there is crossing over of some of the curves corresponding to different normalized ion temperature $T_{i} / T_{e}$ (Figure 5.9(c) has been repeated to show the labelling of the individual curves). This behaviour becomes clearer by examining the growth rate as a function of the normalized equilibrium density $n_{e o} / n_{o}$ for (a) $k \lambda_{d}=1.0$ and (b) $k \lambda_{d}=2.0$ (Figure 5.10). For fixed $k \lambda_{d}=1.0$, there is no growth beyond $n_{e o} \approx 0.8 n_{o}$. For normalized wave number fixed at 2.0 , the behaviour of the curves are as for the three-component plasma of Bharuthram et al. (1996) in that there is no crossing over.

Figure 5.11 shows the effect of increasing the normalized ion temperature $T_{i} / T_{e}$ on the instability growth rate. As $T_{i} / T_{e}$ increases, the growth rate decreases as found for the single dust component plasmas in Chapter 4. This reduction in the growth rate is attributed to the kinetic nature of the dust-acoustic instability as explained for the three-component plasmas of the previous chapter. The corresponding real frequencies are shown in Figure 5.12. Figure 5.13 depicts the comparison of the numerical solution of real frequency with the approximate solution (5.3) for different values of $T_{i} / T_{e}$. It is noted that the agreement is better for higher values of normalized ion temperature. This is to be expected since one of the assumptions in deriving the approximate solution (5.3) is that $T_{i} \approx T_{e} \gg T_{d h}, T_{d l}$. For $T_{i}=T_{e}$, the numerical real frequency is compared with the approximate solution for different values of the equilibrium electron density (Figure 5.14), done for values of $k$ for which $\gamma>0$. 
(a)
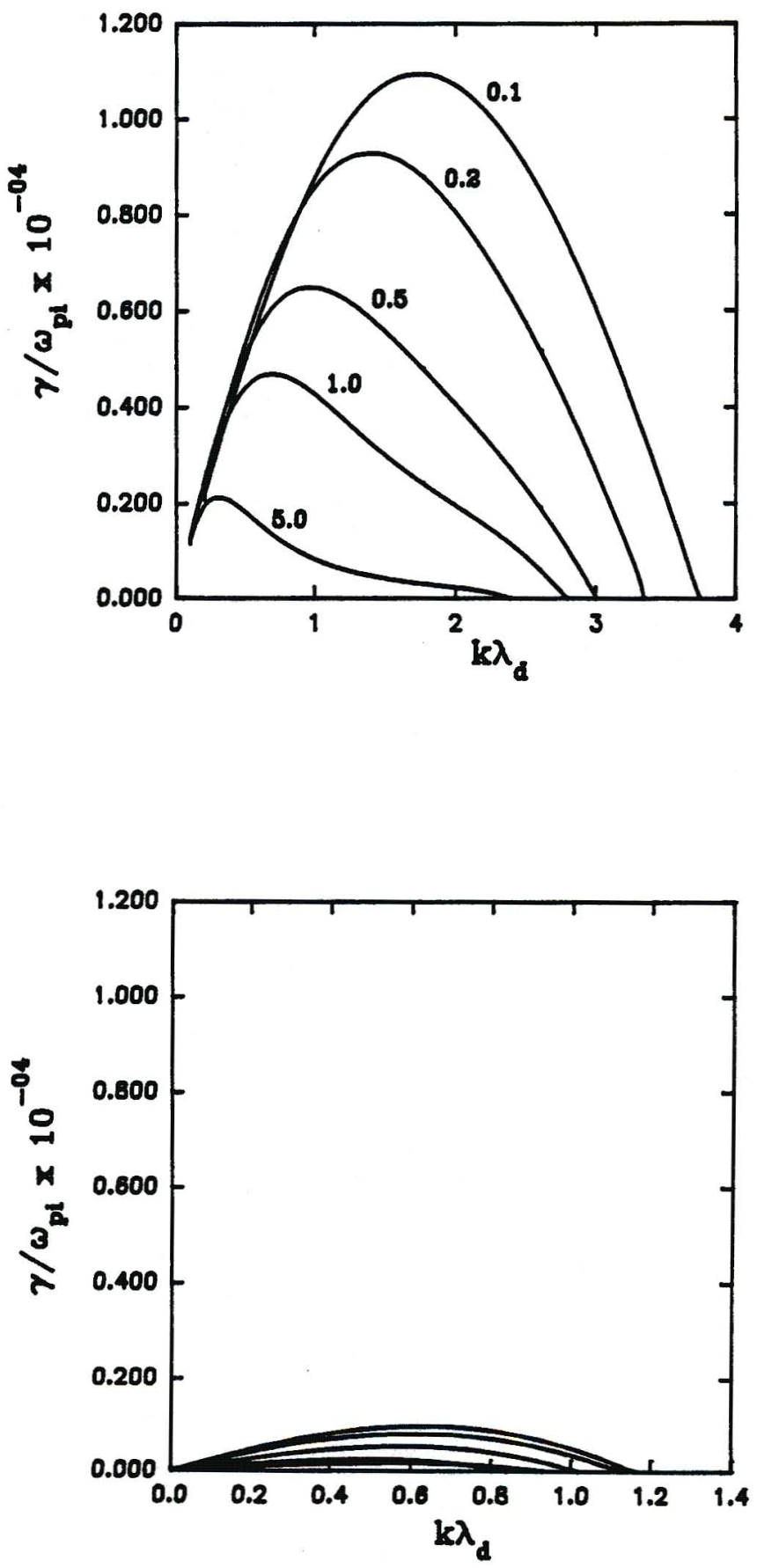

(c)

(b)
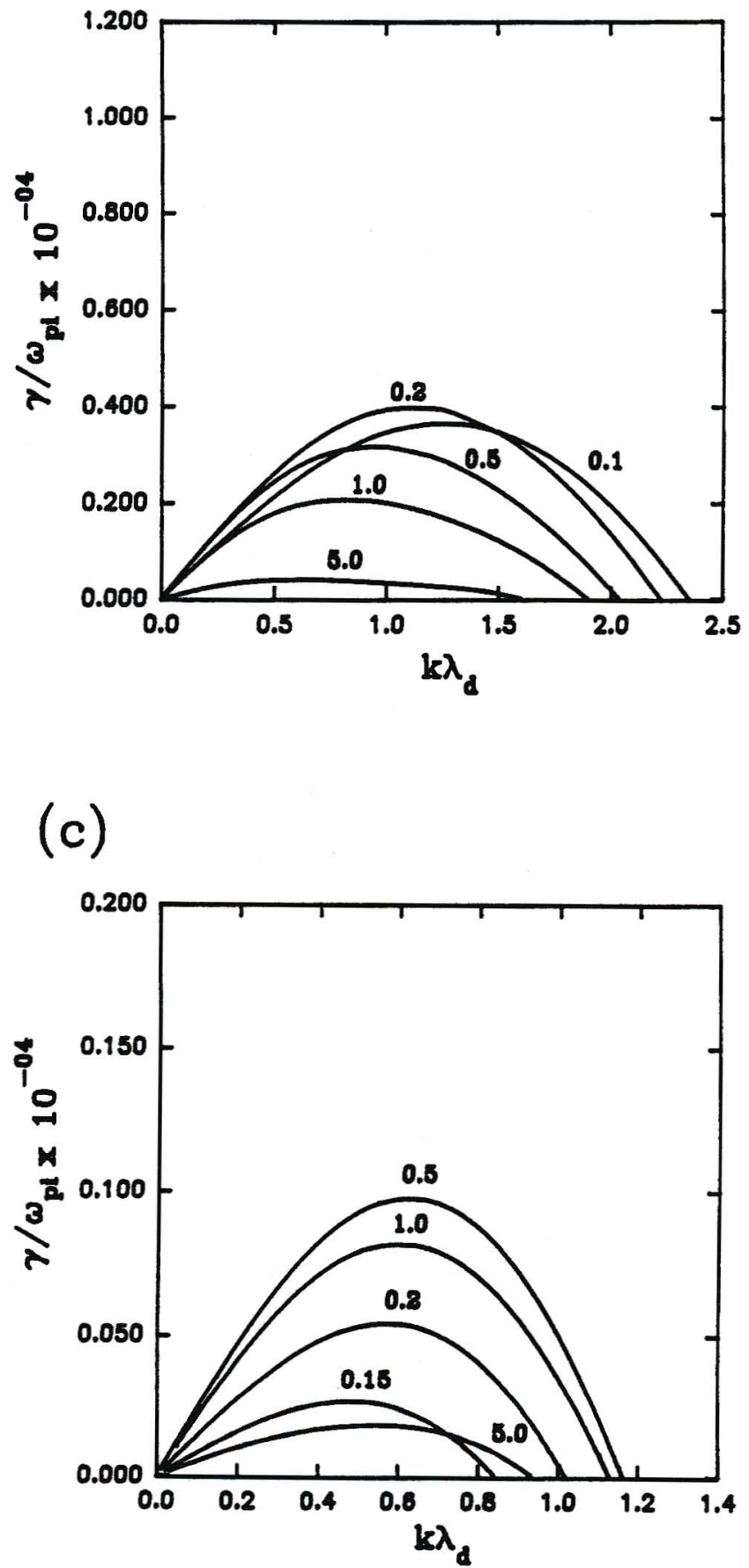

Figure 5.9: Plot of the normalized growth rate versus $k \lambda_{d}$ for $V_{d i z}=0.2 C_{s}$ for (a) $n_{e o}=0.00 n_{o}$, (b) $n_{e o}=0.5 n_{o}$ and (c) $n_{e o}=0.75 n_{o}$. The parameter labelling the curves is the normalized ion temperature $T_{i} / T_{e}$. All other fixed parameters are as in Figure 5.1 
(1)

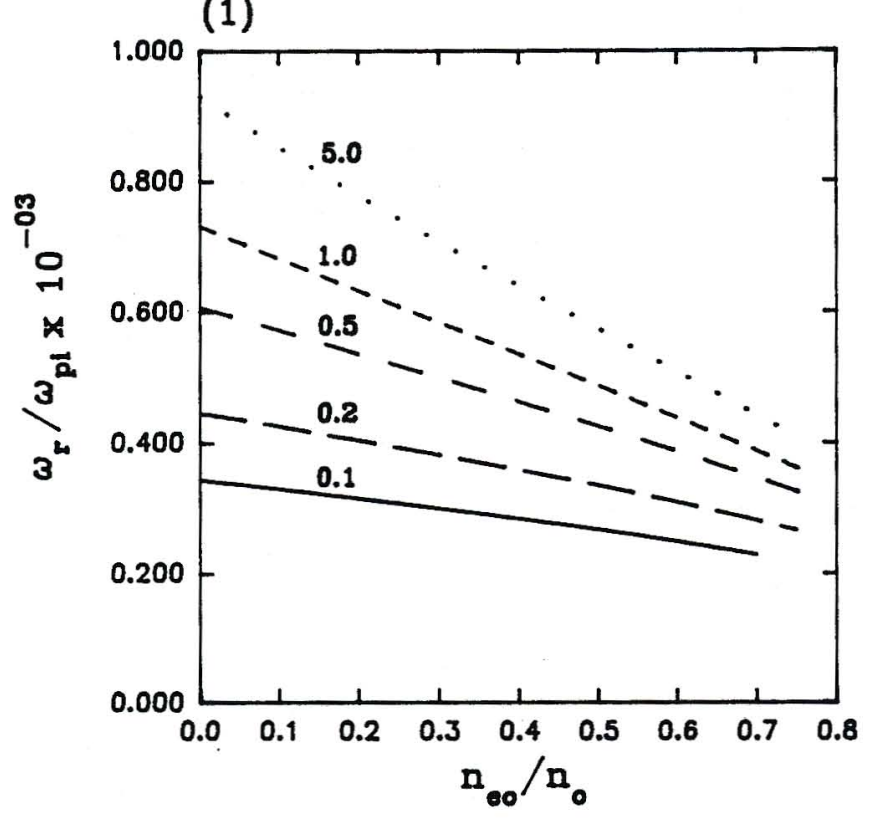

(1)

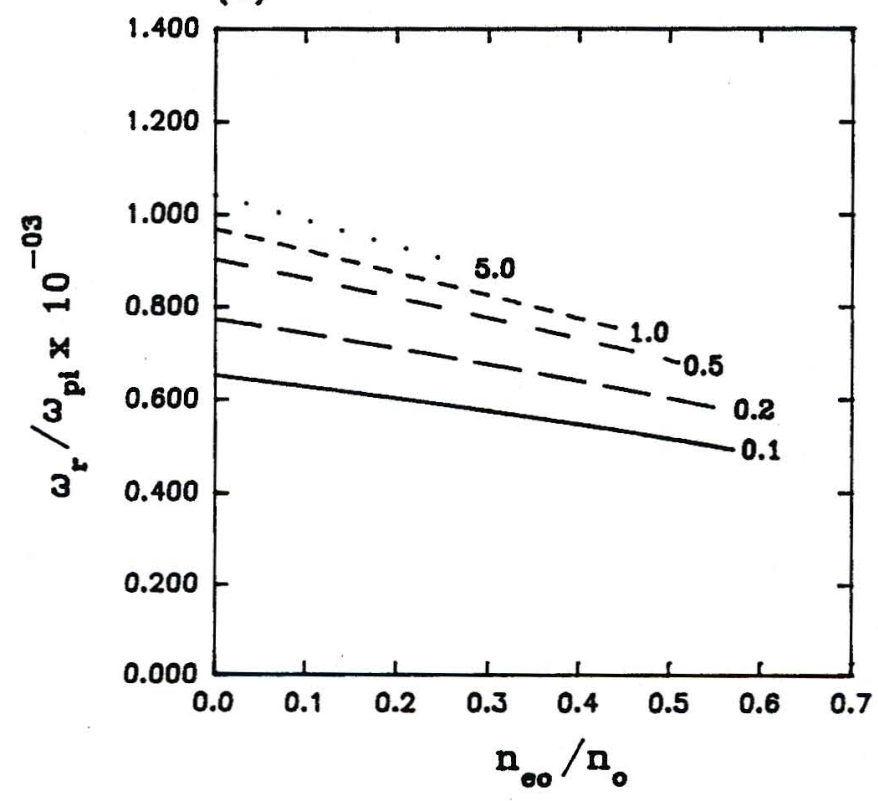

(a)

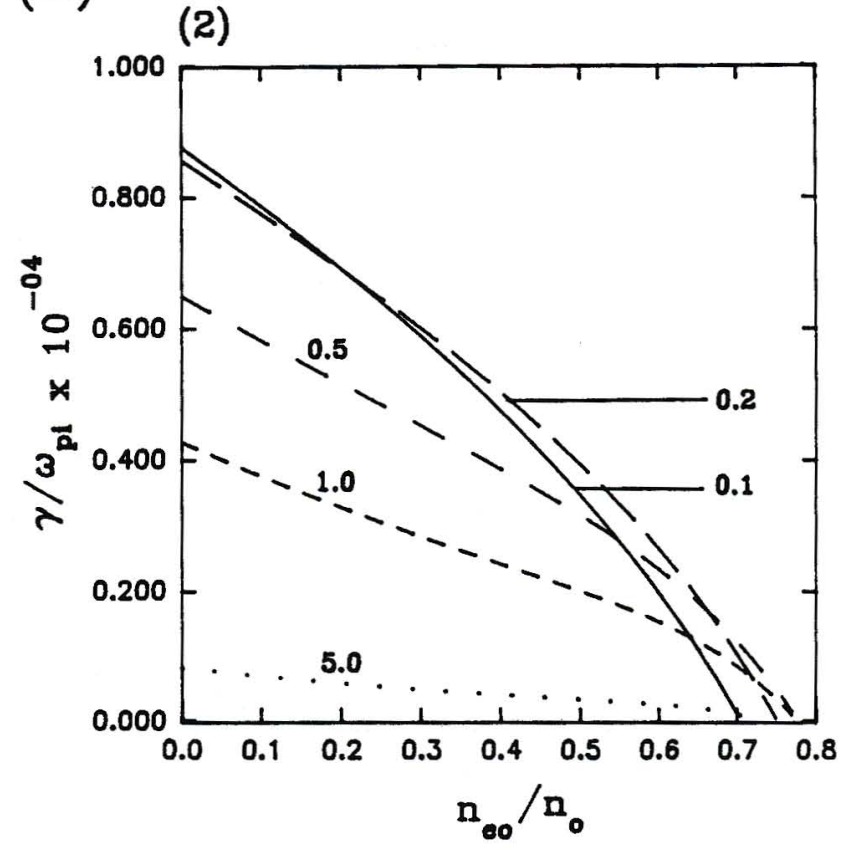

(b)

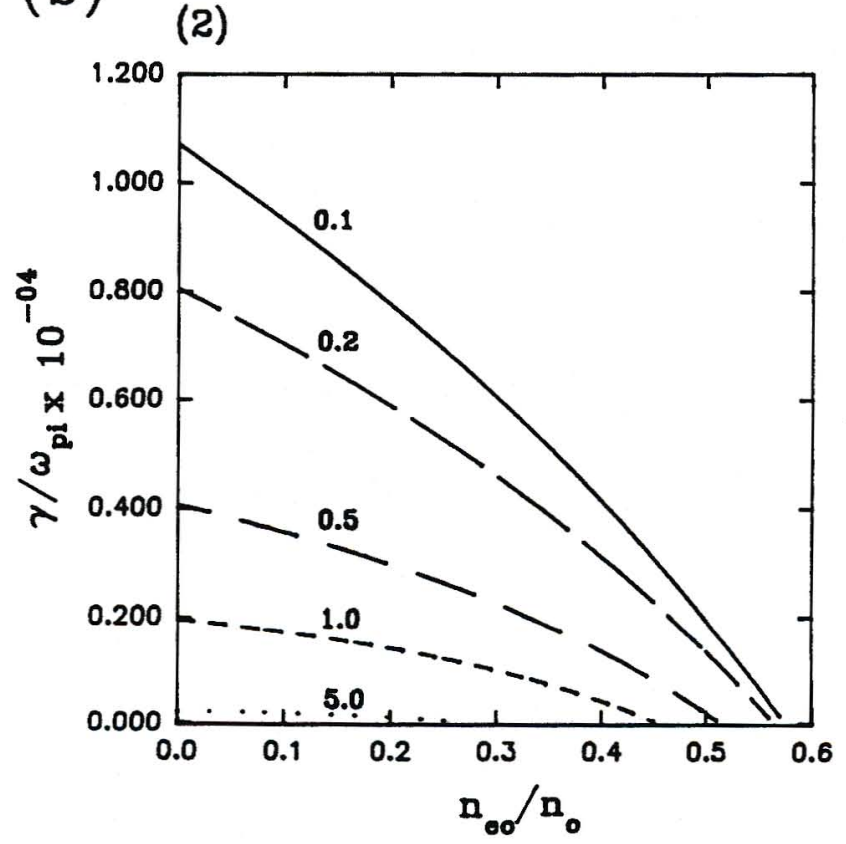

Figure 5.10: Plot of the normalized real frequency (1) and growth rate (2) versus $n_{e o} / n_{o}$ for (a) $k \lambda_{d}=1.0$ and (b) $k \lambda_{d}=2.0$. The parameter labelling the curves is $T_{i} / T_{e}$. All other fixed parameters are as in Figure 5.1 


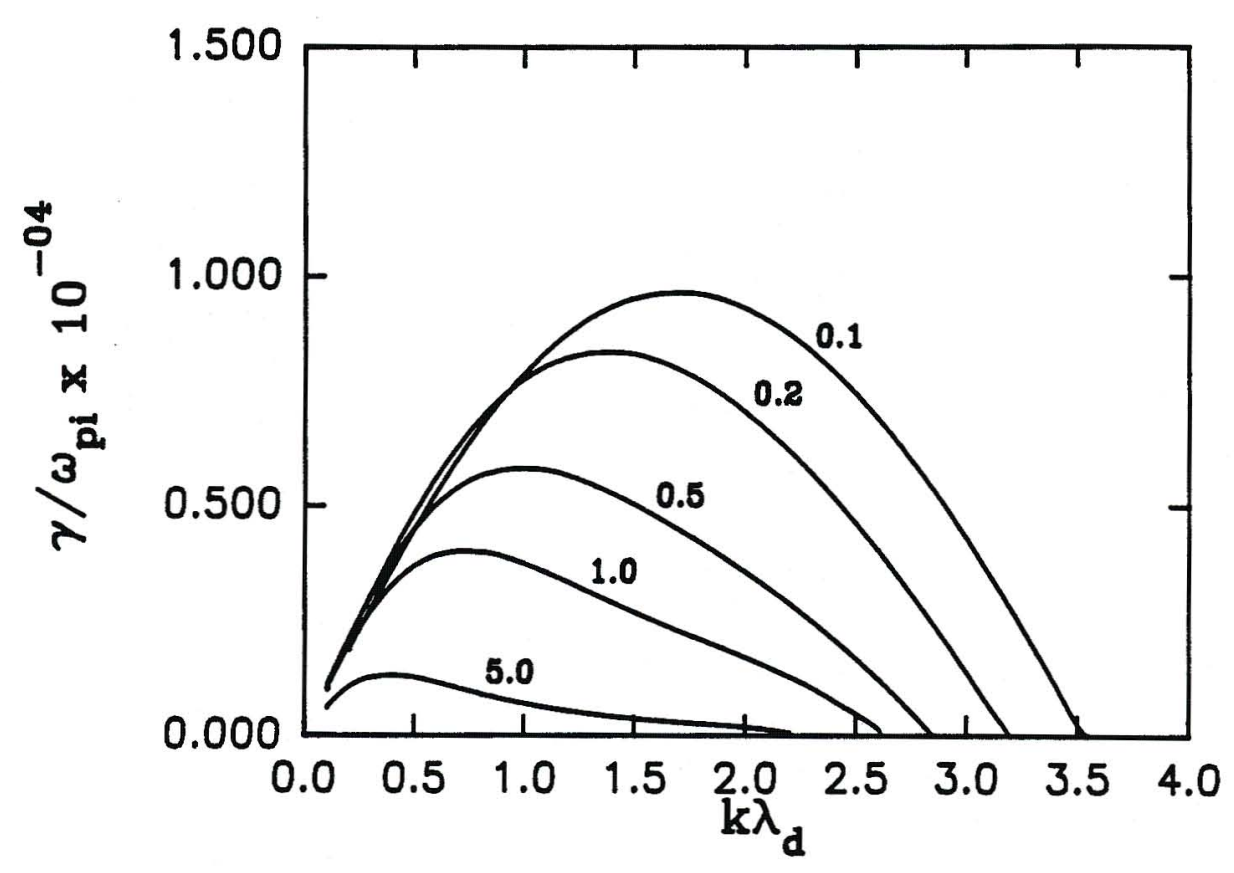

Figure 5.11: Plot of the normalized growth rate versus $k \lambda_{d}$ for $V_{d i z}=0.2 C_{s}$. The parameter labelling the curves is $T_{i} / T_{e}$. All other fixed parameters are as in Figure 5.1

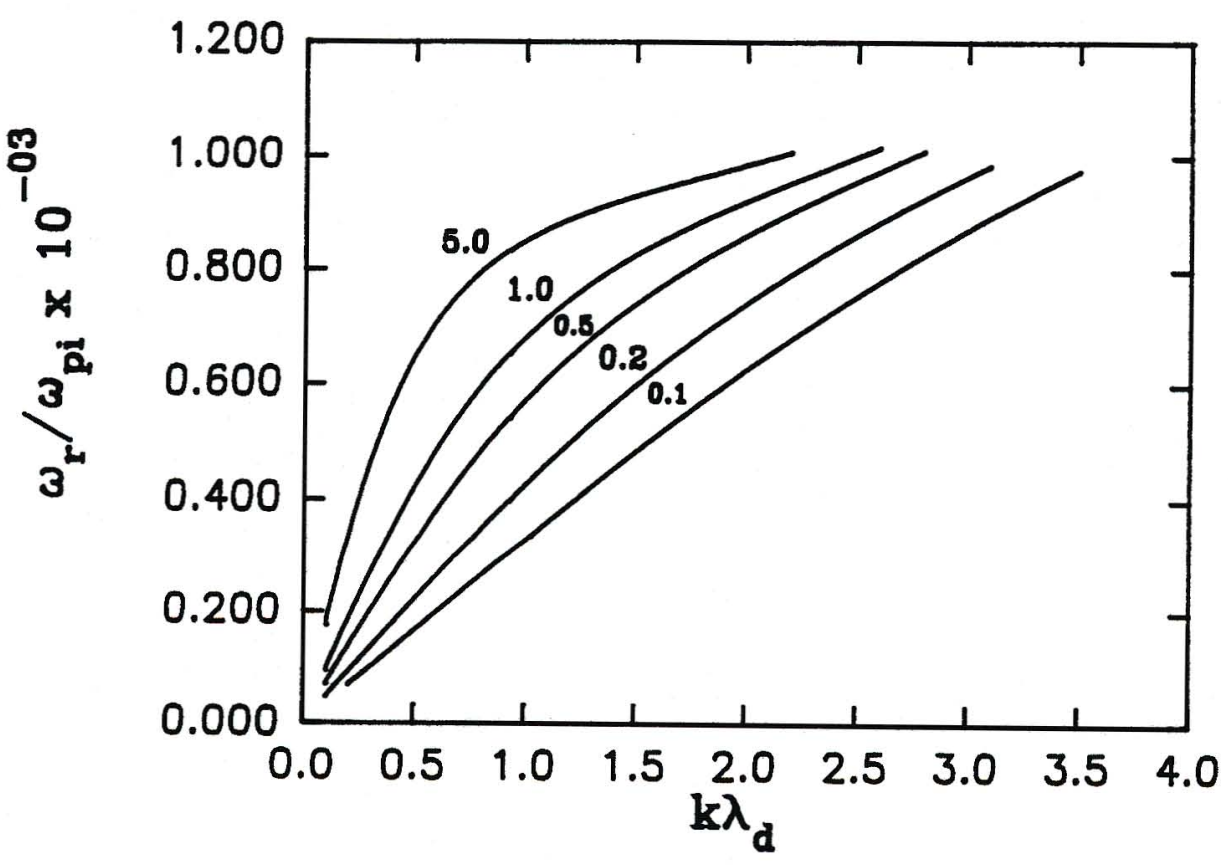

Figure 5.12: Plot of normalized real frequencies corresponding to the growth rates of Figure 5.11. The parameter labelling the curves is $T_{i} / T_{e}$ 


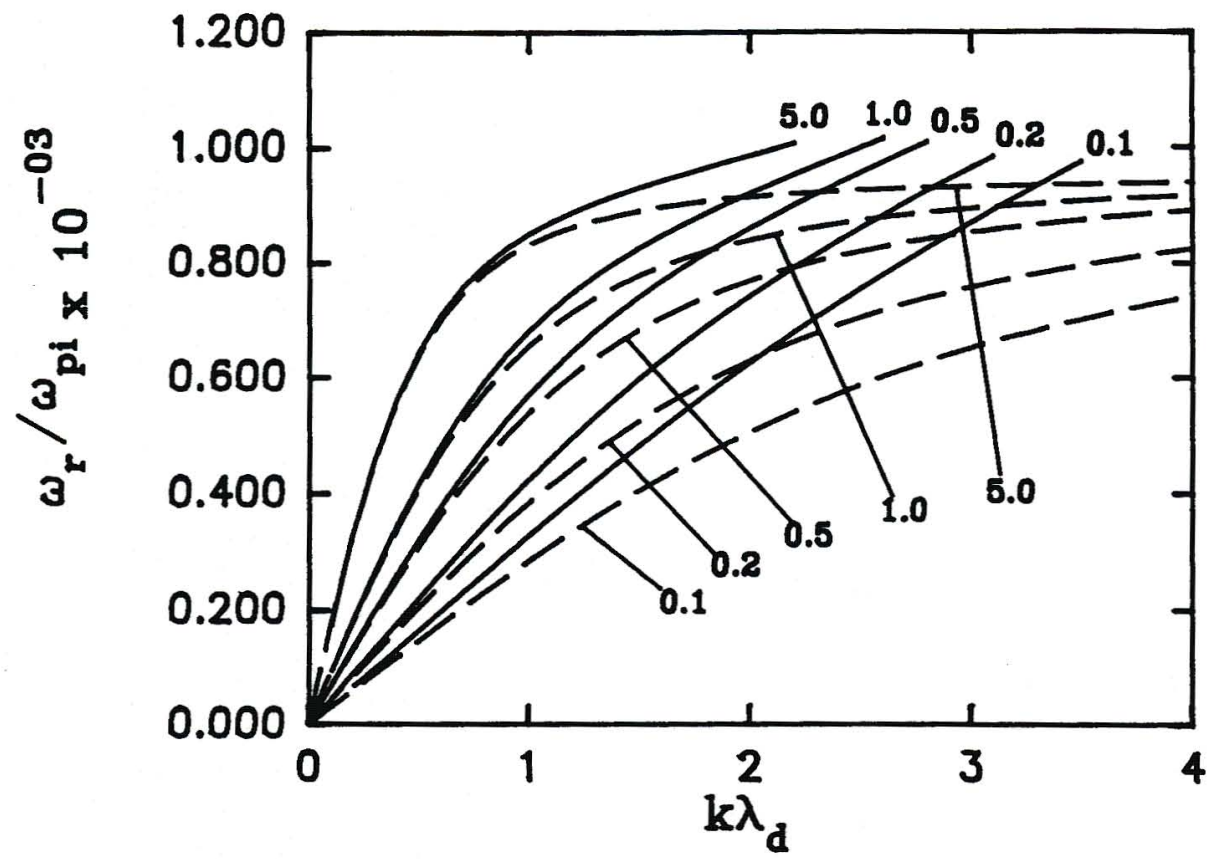

Figure 5.13: Plot of the normalized real frequency versus $k \lambda_{d}$ for $V_{d i z}=0.2 C_{s}$. The parameter labelling the curves is $T_{i} / T_{e}$. The dashed curves are calculated from the approximate expression (5.3). All other fixed parameters are as in Figure 5.1

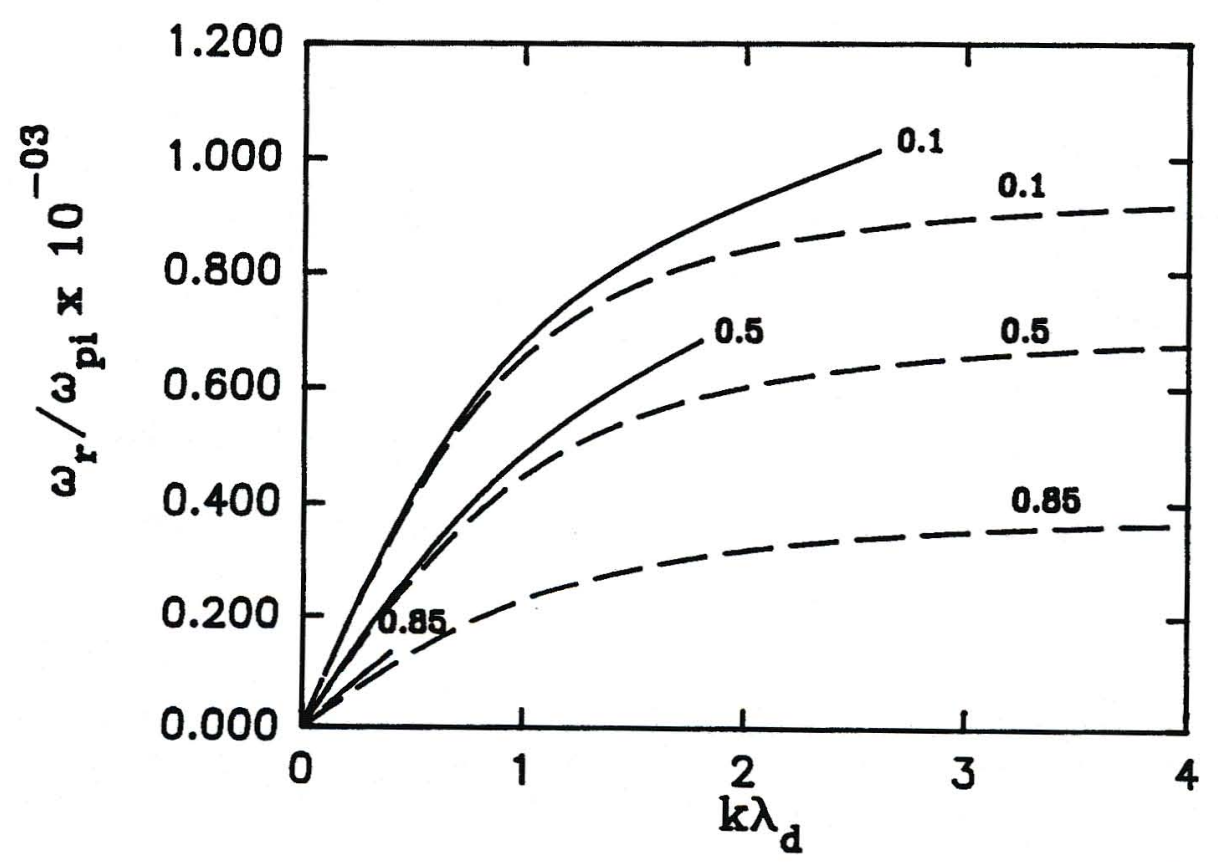

Figure 5.14: Plot of normalized real frequency as a function of $k \lambda_{d}$ for $V_{d i z}=0.2 C_{s}$. The parameter labelling the curves is the normalized electron density $n_{e o} / n_{o}$. Here $T_{i}=T_{e}$. The dashed curves are calculated from the approximate expression (5.3). All other fixed parameters are as in Figure 5.1 
We finally study the effect of the magnetic field strength on the instability. It is seen in Figure 5.15 that as the magnitude of $B_{\circ}$ increases, the range of propagation angles for which there is positive growth widens, and the growth rates are enhanced as observed for the single dust constituent plasmas in the previous chapter. The growth rate $\gamma$ is again a maximum for propagation along $\mathbf{B}_{\mathbf{o}}$ since this is the direction of wave propagation for maximum resonance with the ion beam.

Finally the effect of selected oblique angles of wave propagation relative to the magnetic field $\mathbf{B}_{\mathbf{o}}$ is examined. This is depicted for angles $\theta=\sin ^{-1}\left(k_{y} / k\right)=45^{\circ}$ (Figures 5.16-5.18), $30^{\circ}$ (Figures 5.19-5.21) and $72^{\circ}$ (Figures 5.22-5.24). The mode obtained for $\omega_{p i} / \Omega_{i}=0.01$ is the dust-acoustic mode shown in Figures 5.16, 5.19 and 5.22. It is noted that for values of $\omega_{p i} / \Omega_{i}$ of 0.5 (Figures 5.17, 5.20 and 5.23) and 1.0 (Figures 5.18, 5.21 and 5.24), two modes are obtained. This second mode present only for propagations oblique to $\vec{B}_{o}$, corresponds to the dust-modified two-stream instability (DMTSI) first studied by Rosenberg and Krall (1995) and later by Bharuthram (1997). For $k_{y} / k=0.5$ and $\omega_{p i} / \Omega_{i}=0.5$, Figure 5.25 illustrates both solutions for normalized $\omega_{r}$ of the full dispersion relation as well as the approximate solution (5.3) for the purpose of easy comparison. The growth rates corresponding to the real frequency curves in Figure 5.25 are shown in Figure 5.26. 


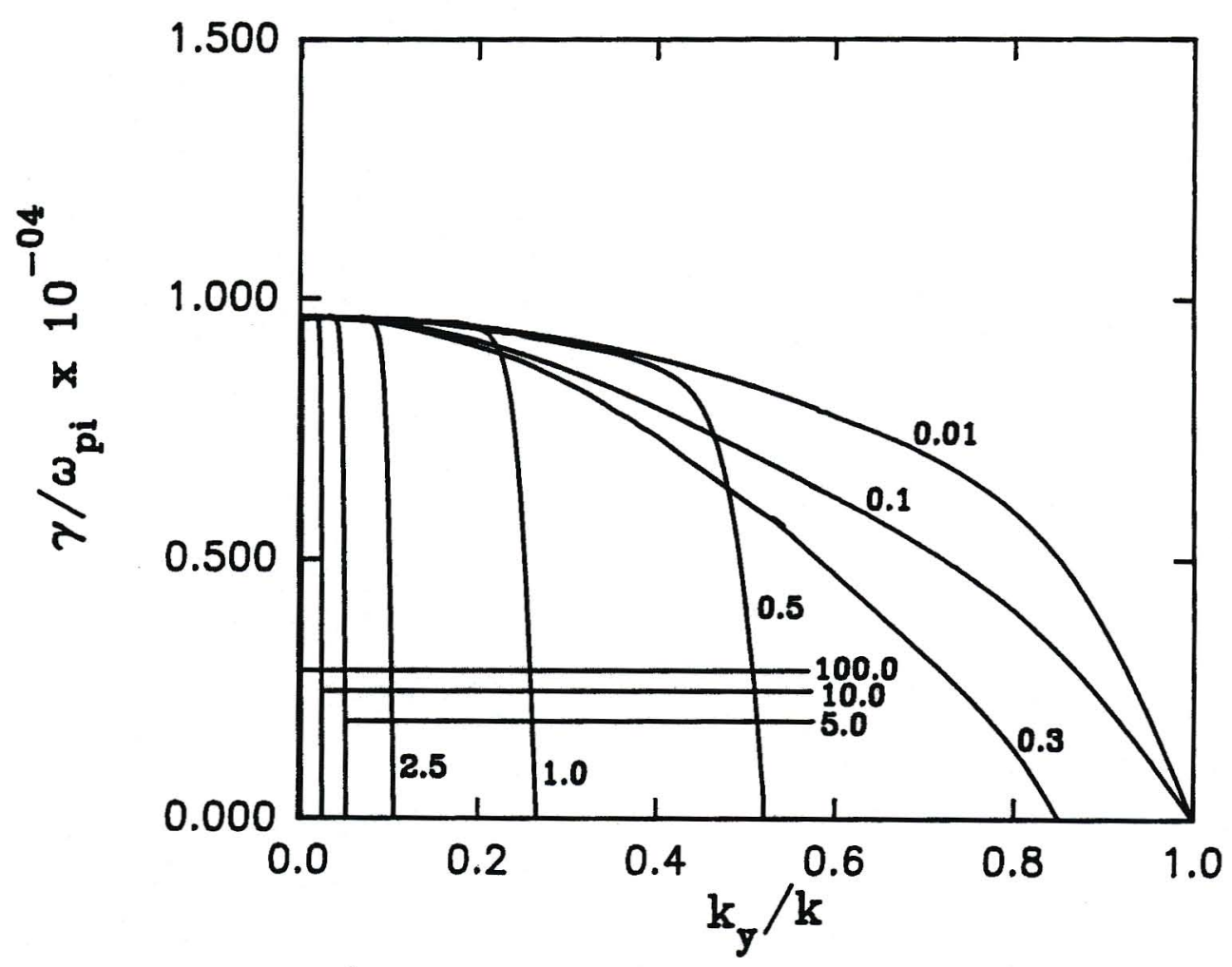

Figure 5.15: Plot of the normalized growth rate versus $k_{y} / k$ for $V_{d i z}=0.2 C_{s}$. Here $T_{i}=0.1 T_{e}$ and $k \lambda_{d}=2.4$. The parameter labelling the curves is $\omega_{p i} / \Omega_{i}$. All other fixed parameters are as in Figure 5.1 


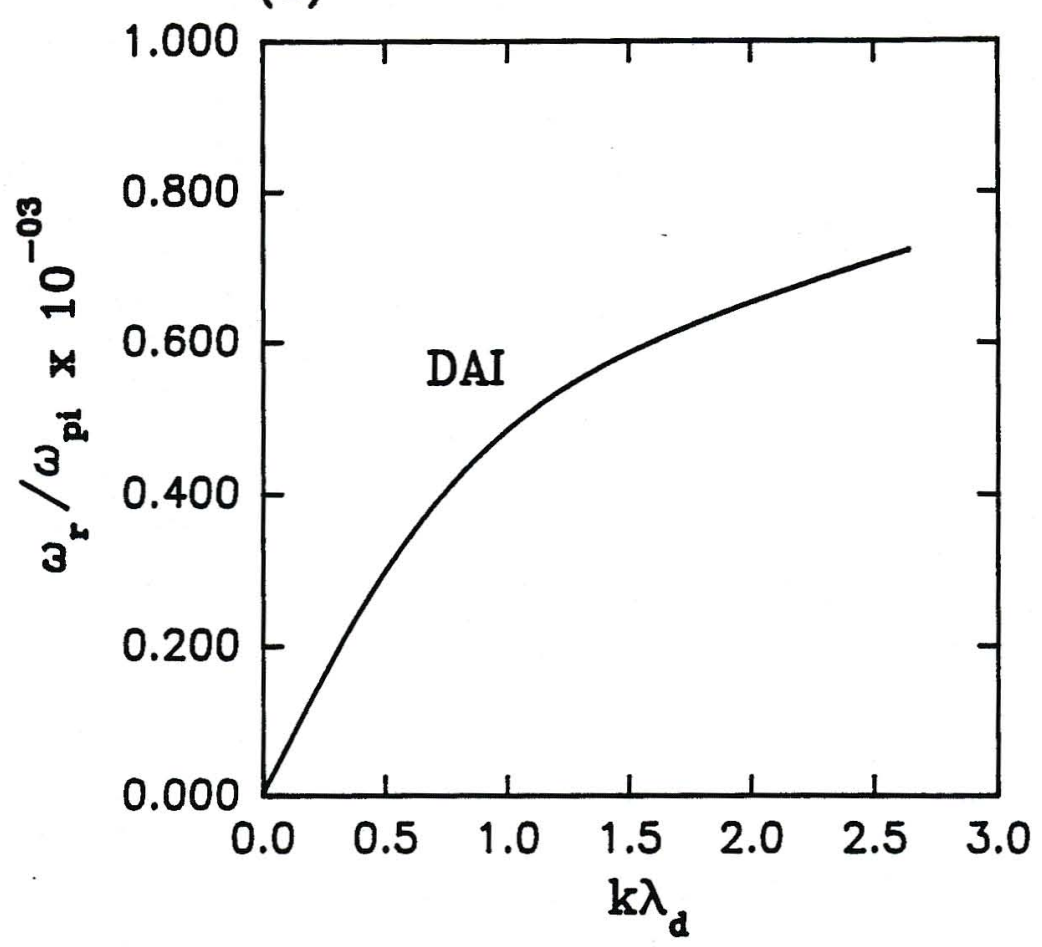

(2)

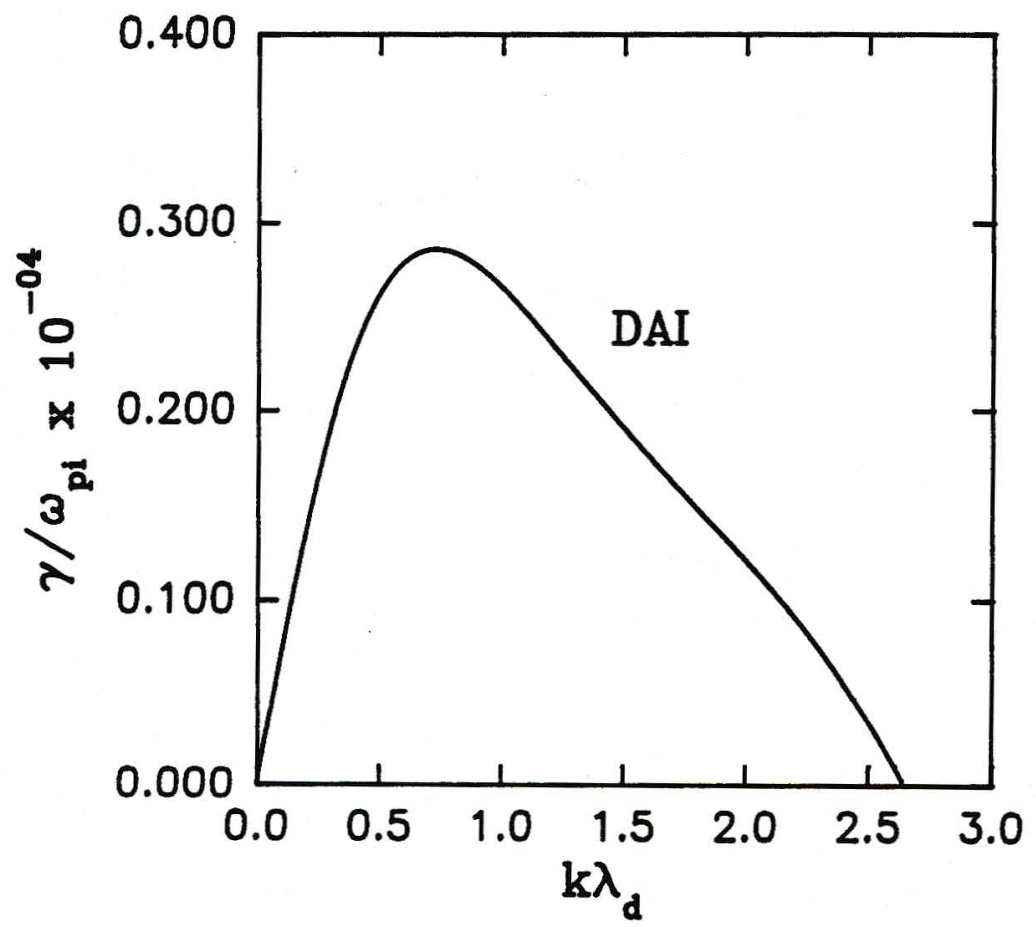

Figure 5.16: Plot of normalized real frequency (1) and growth rate (2) versus $k \lambda_{d}$ for oblique angles of propagation relative to $\mathbf{B}_{\mathbf{o}}$. Here $T_{i}=T_{e}, k_{y} / k=0.707\left(\theta=45^{\circ}\right)$ and $\omega_{p i} / \Omega_{i}=0.01$. All other fixed parameters are as in Figure 5.15 
(1)

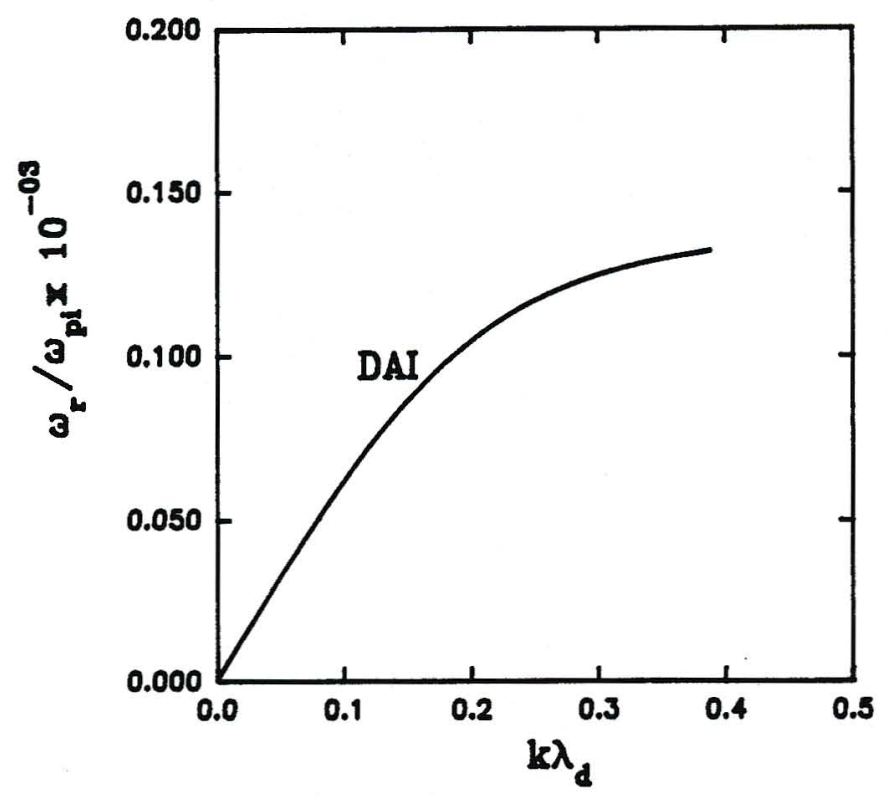

(1)

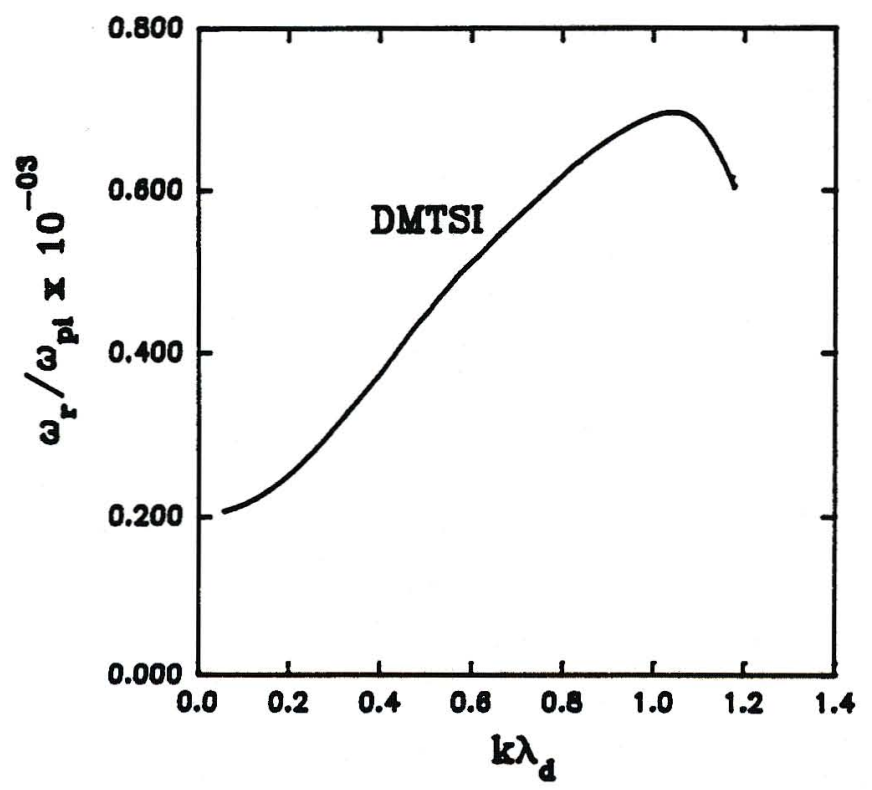

(2)

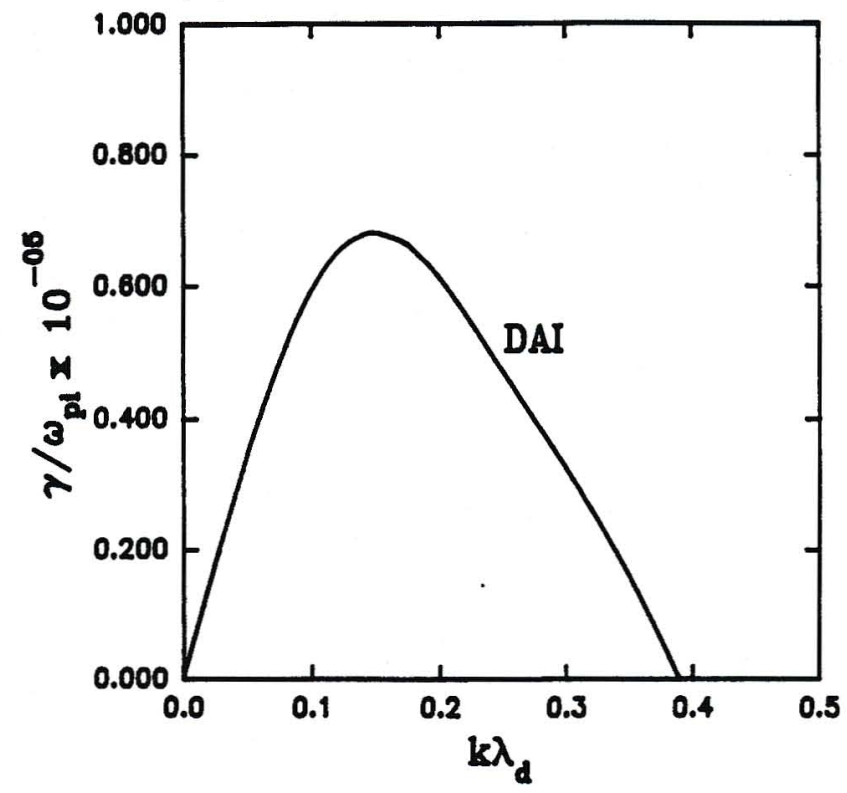

(2)

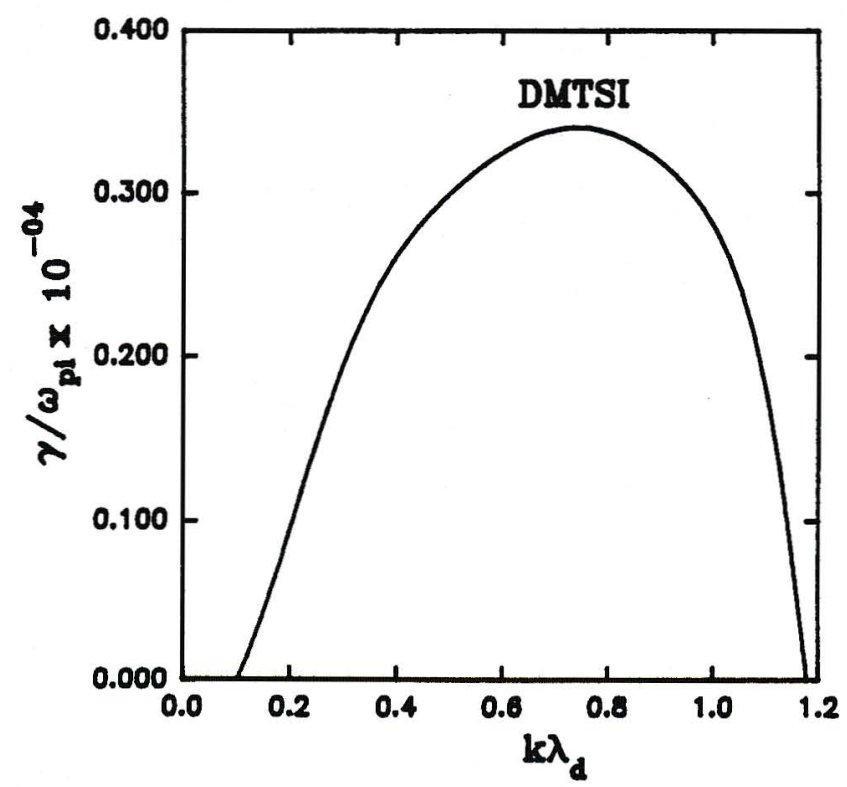

Figure 5.17: Plot of normalized real frequency (1) and growth rate (2) versus $k \lambda_{d}$ for $k_{y} / k=0.707\left(\theta=45^{\circ}\right)$. Here $\omega_{p i} / \Omega_{i}=0.5$. All other fixed parameters are as in Figure 5.15 
(1)

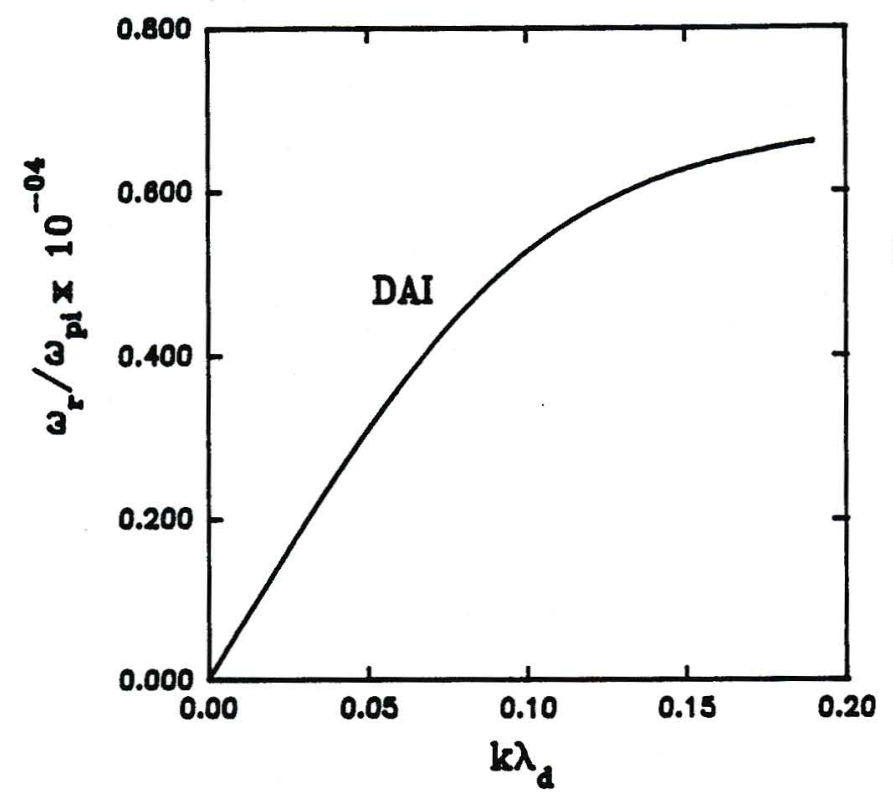

(1)

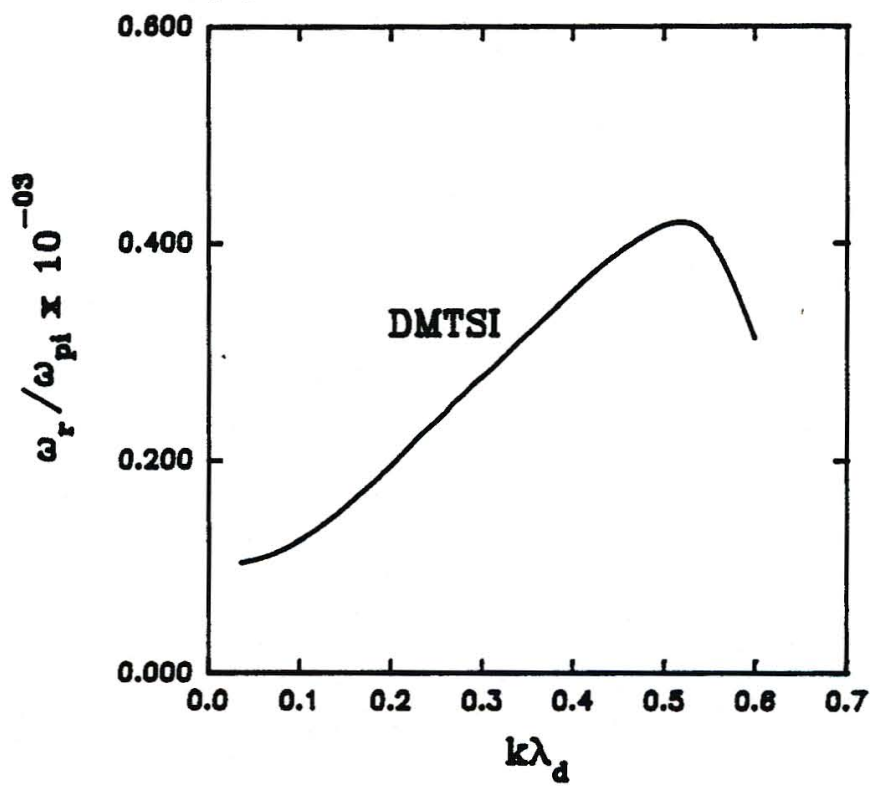

(2)

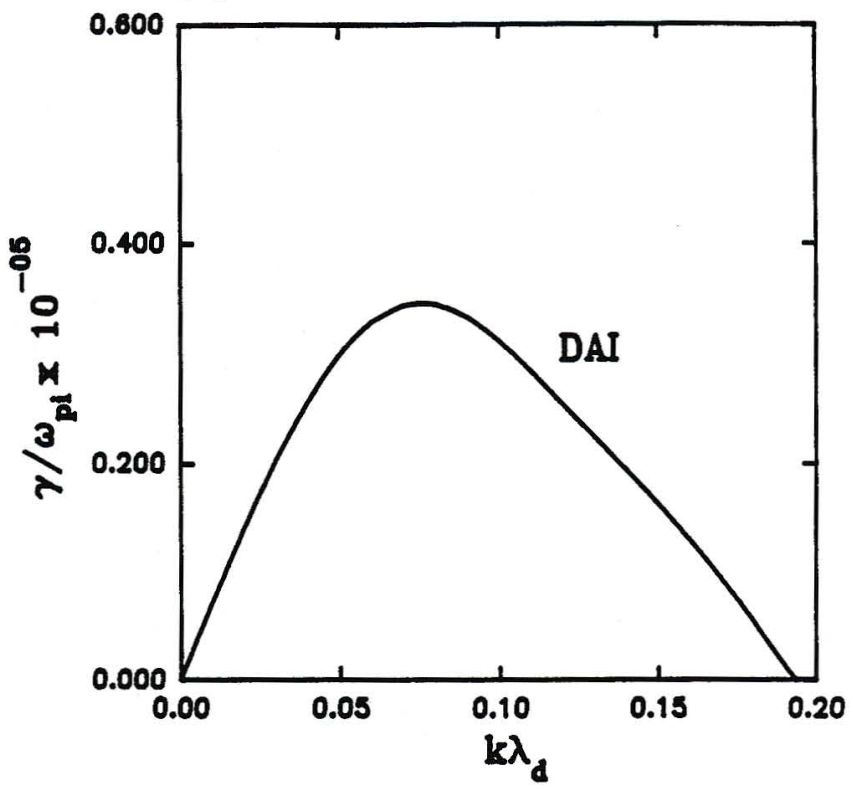

(2)

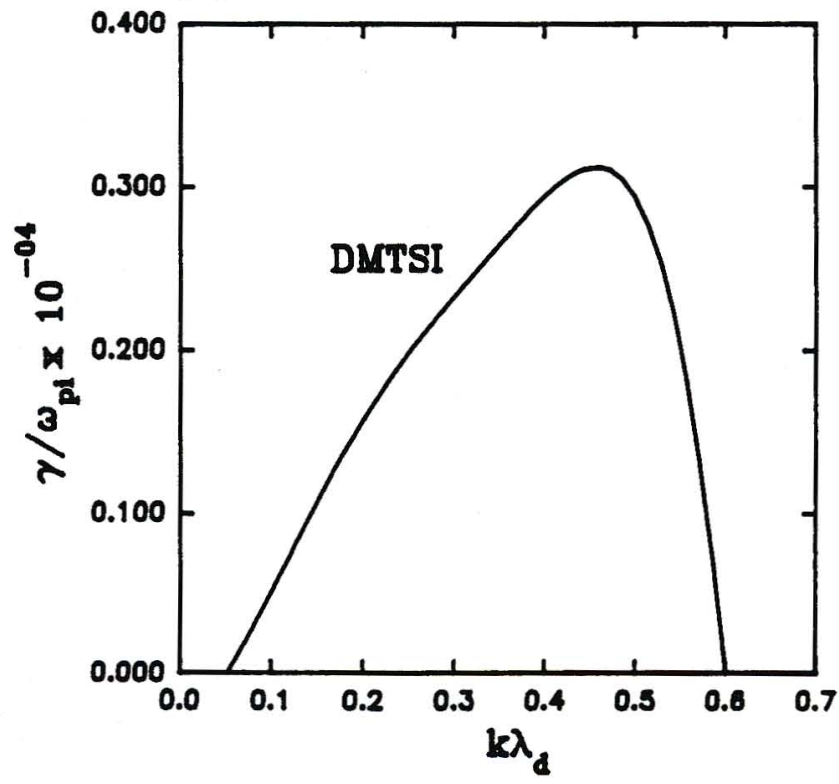

Figure 5.18: Plot of normalized real frequency (1) and growth rate (2) versus $k \lambda_{d}$ for $k_{y} / k=0.707\left(\theta=45^{\circ}\right)$. Here $\omega_{p i} / \Omega_{i}=1.0$. All other fixed parameters are as in Figure 5.15 
(1)

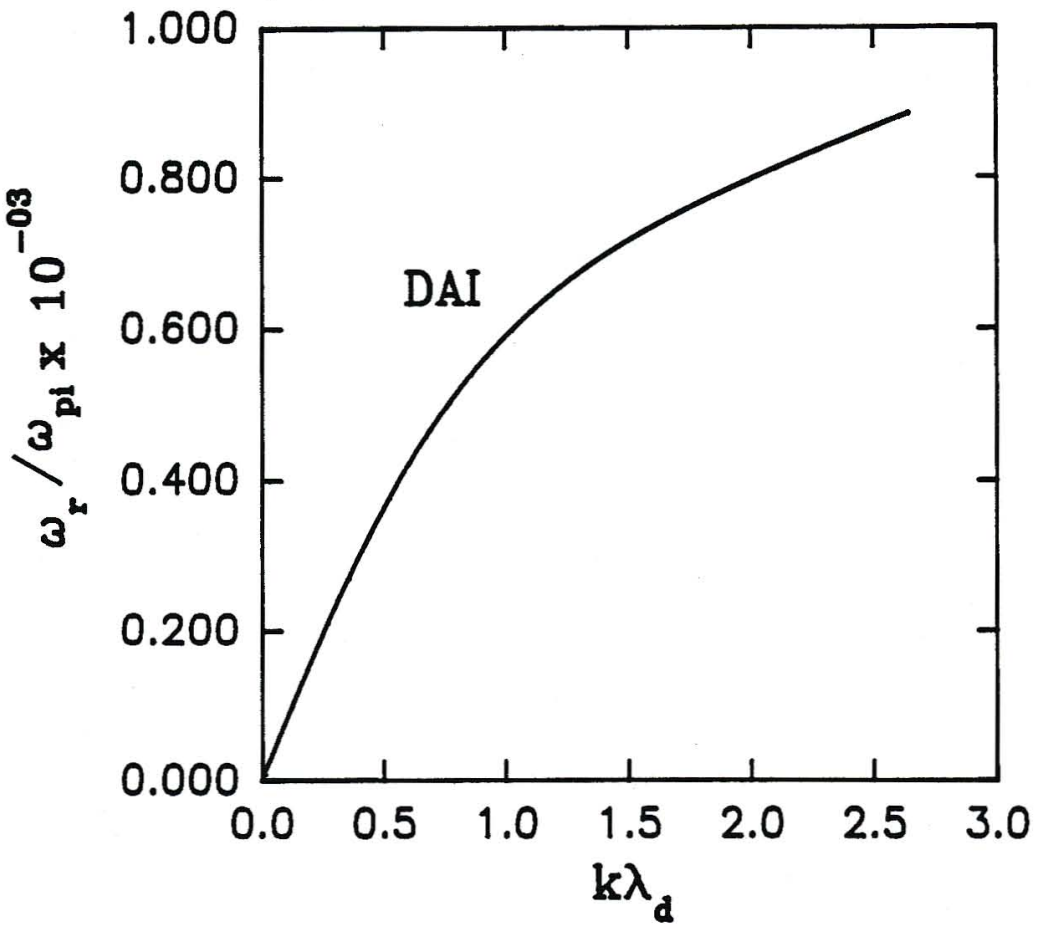

(2)

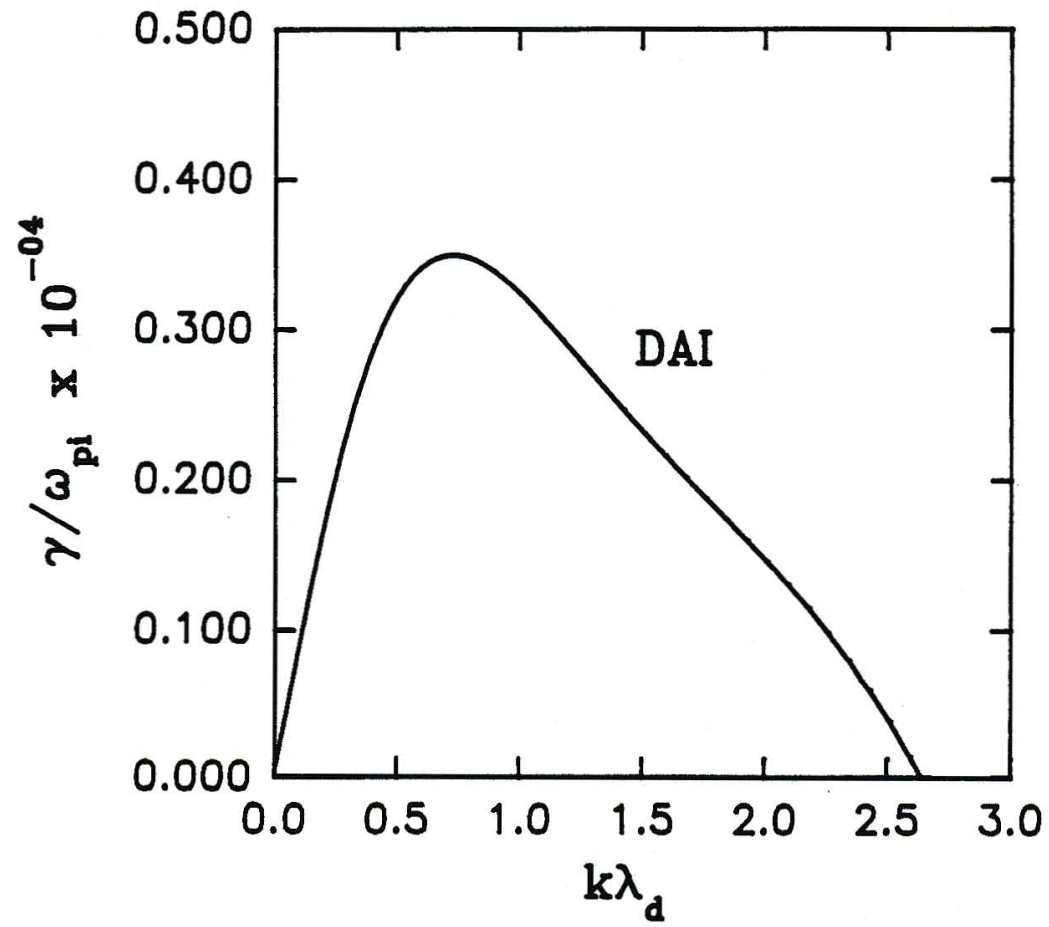

Figure 5.19: Plot of normalized real frequency (1) and growth rate (2) versus $k \lambda_{d}$ for oblique angles of propagation relative to $\mathbf{B}_{\mathbf{o}}$. Here $T_{i}=T_{e}, k_{y} / k=0.5\left(\theta=30^{\circ}\right)$ and $\omega_{p i} / \Omega_{i}=0.01$. All other fixed parameters are as in Figure 5.15 
(1)

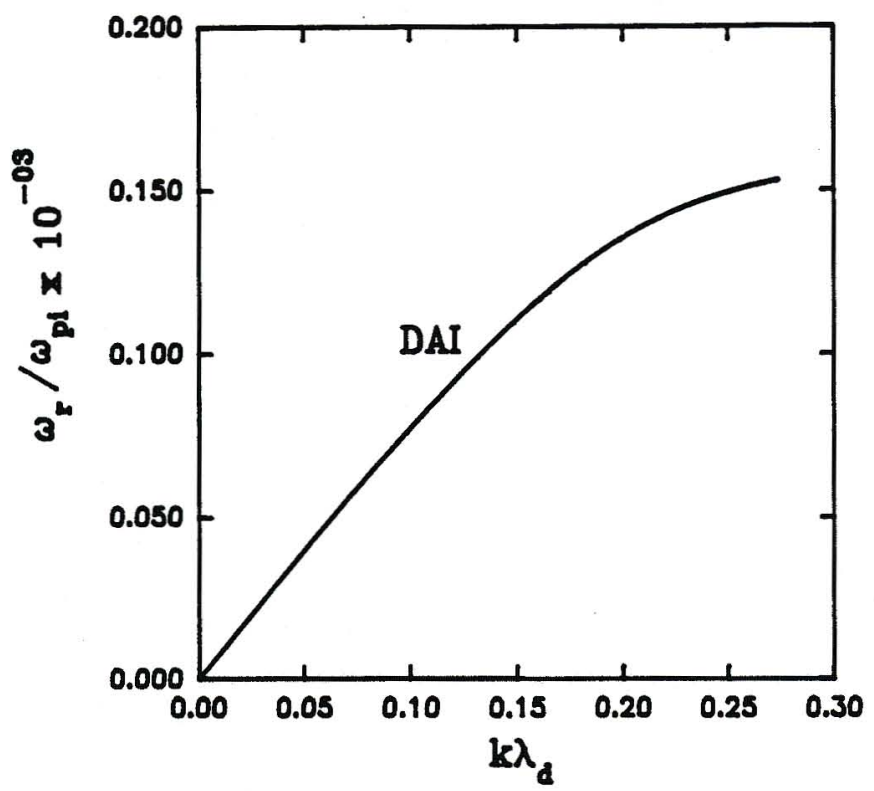

(1)

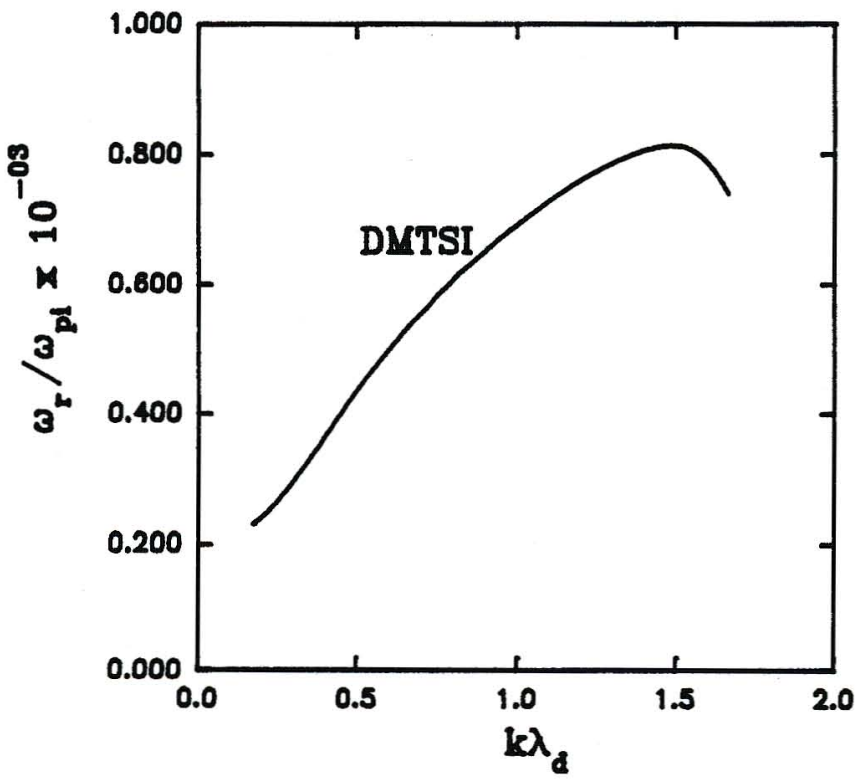

(2)

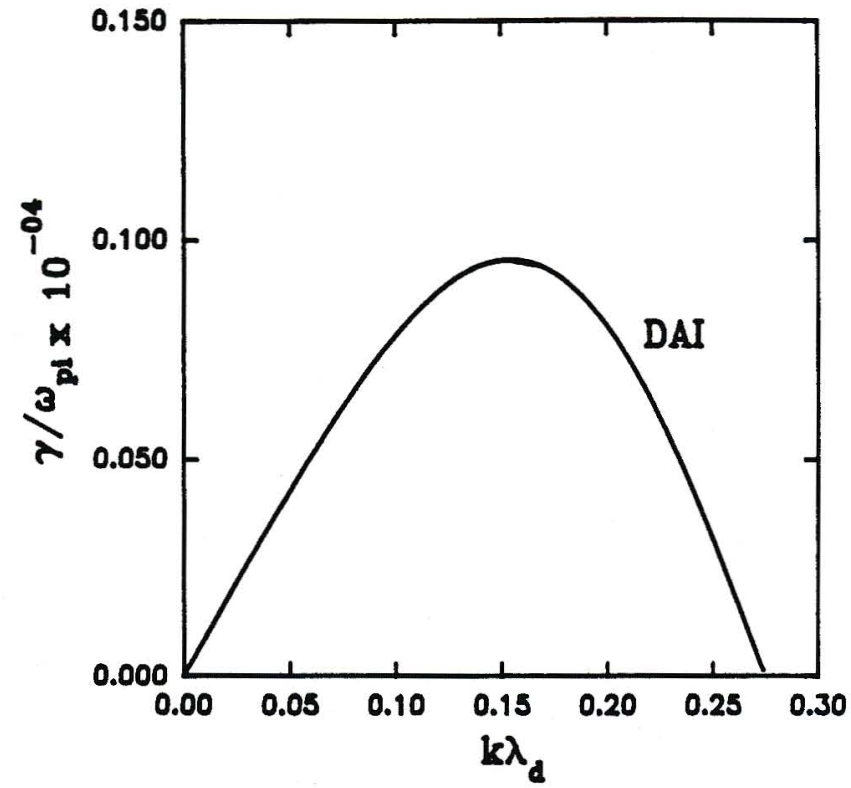

(2)

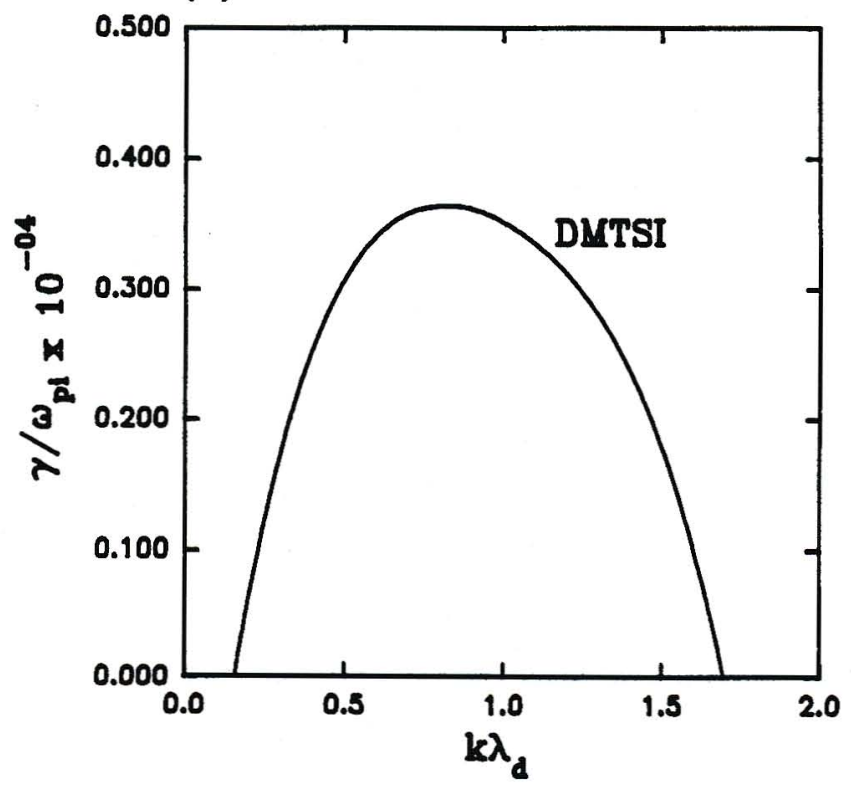

Figure 5.20: Plot of normalized real frequency (1) and growth rate (2) versus $k \lambda_{d}$ for $k_{y} / k=0.5\left(\theta=30^{\circ}\right)$. Here $\omega_{p i} / \Omega_{i}=0.5$. All other fixed parameters are as in Figure 5.15 
(1)

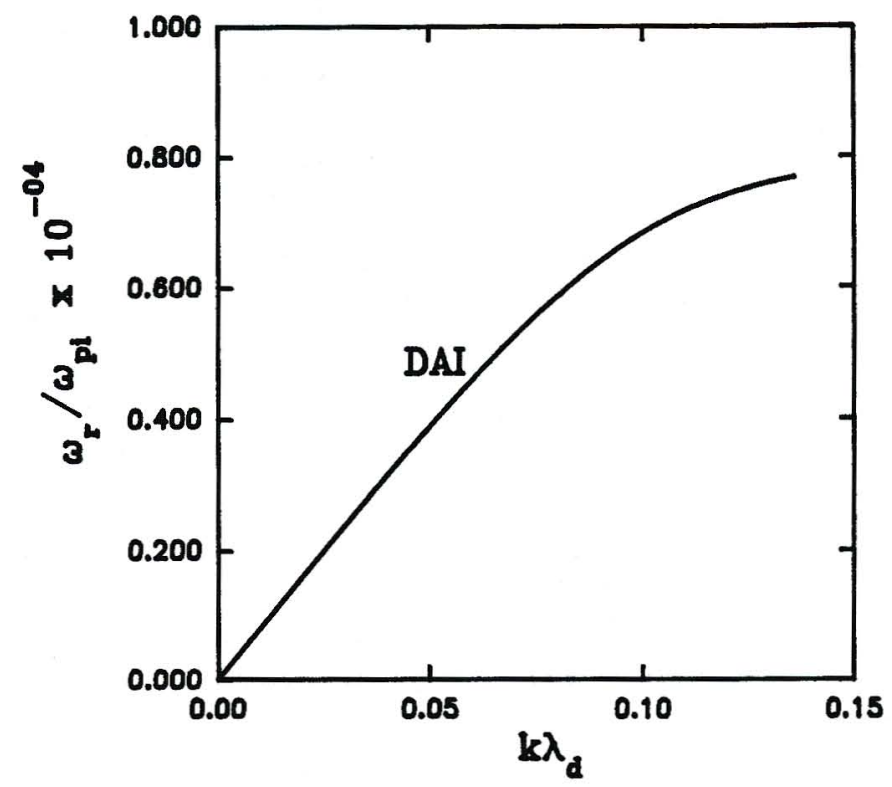

(1)

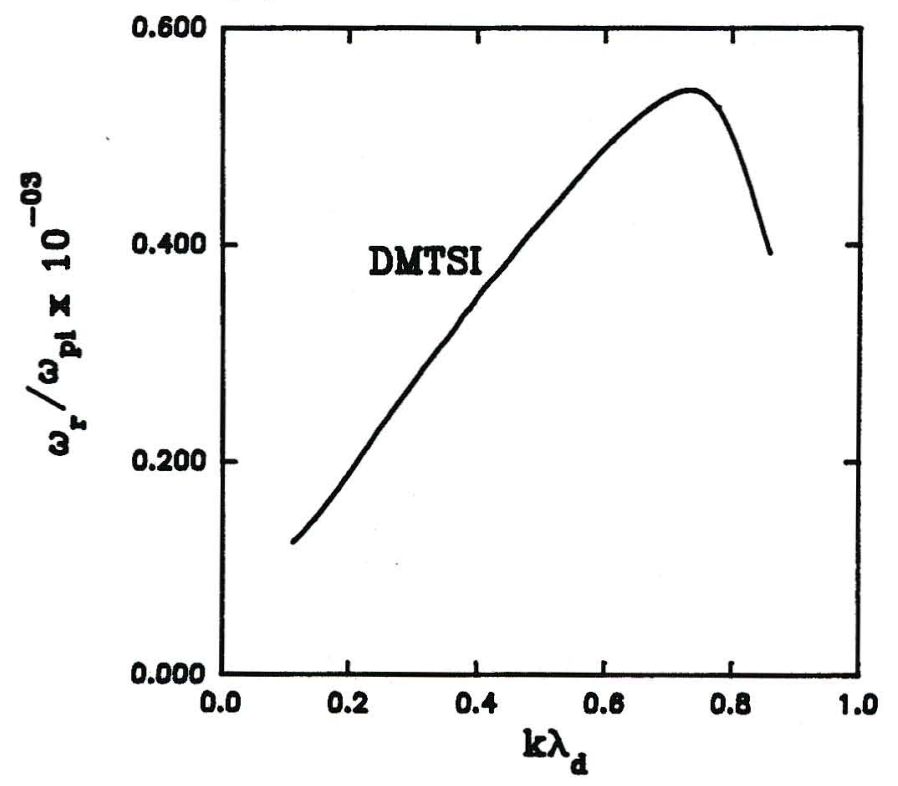

(2)

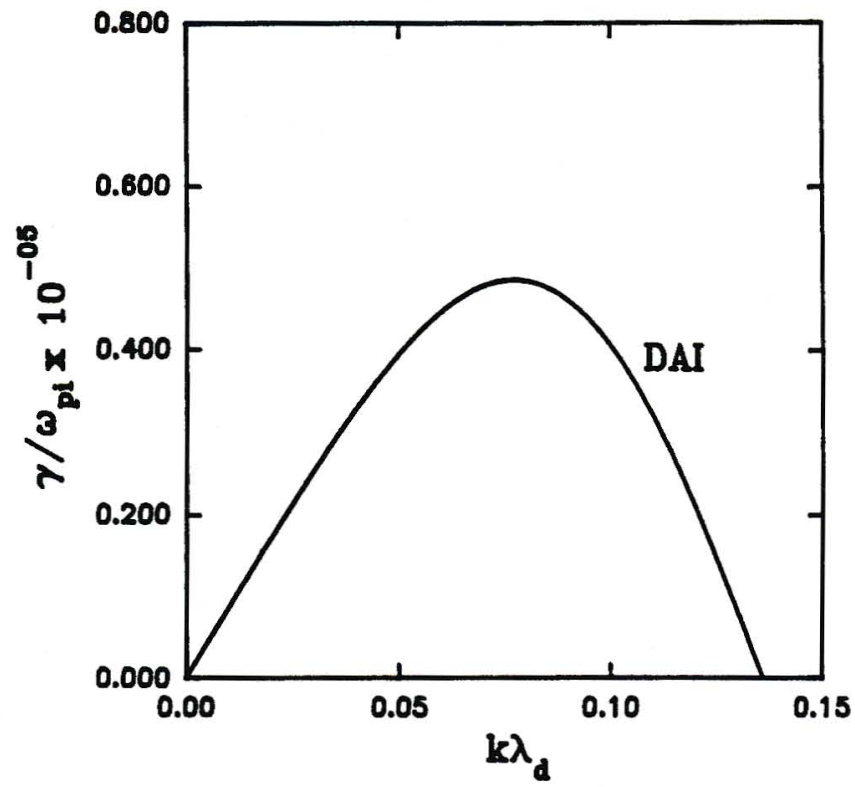

(2)

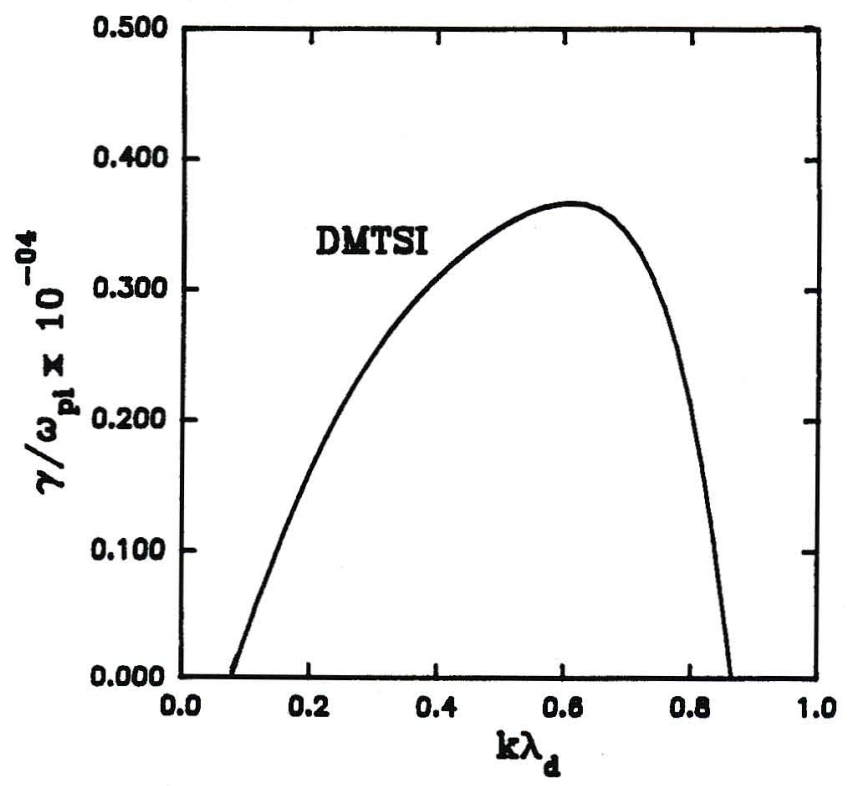

Figure 5.21: Plot of normalized real frequency (1) and growth rate (2) versus $k \lambda_{d}$ for $k_{y} / k=0.5\left(\theta=30^{\circ}\right)$. Here $\omega_{p i} / \Omega_{i}=1.0$. All other fixed parameters are as in Figure 5.15 
(1)

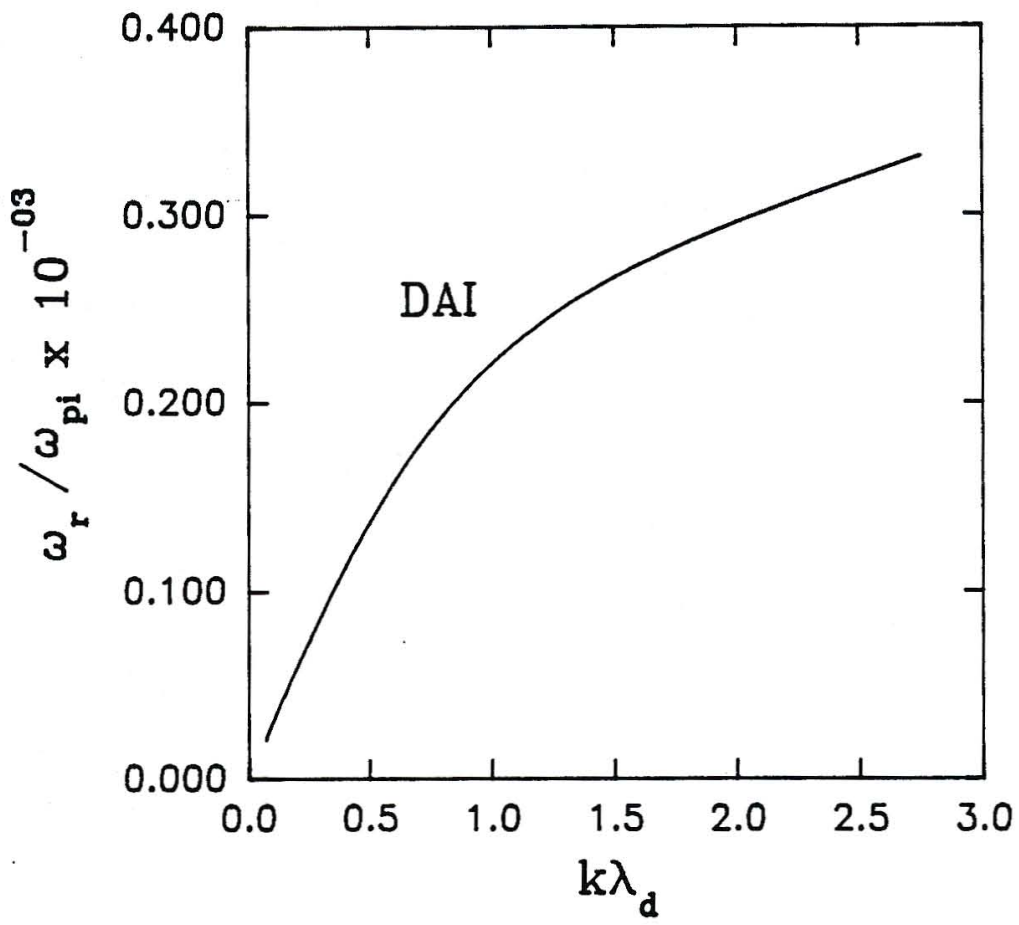

(2)

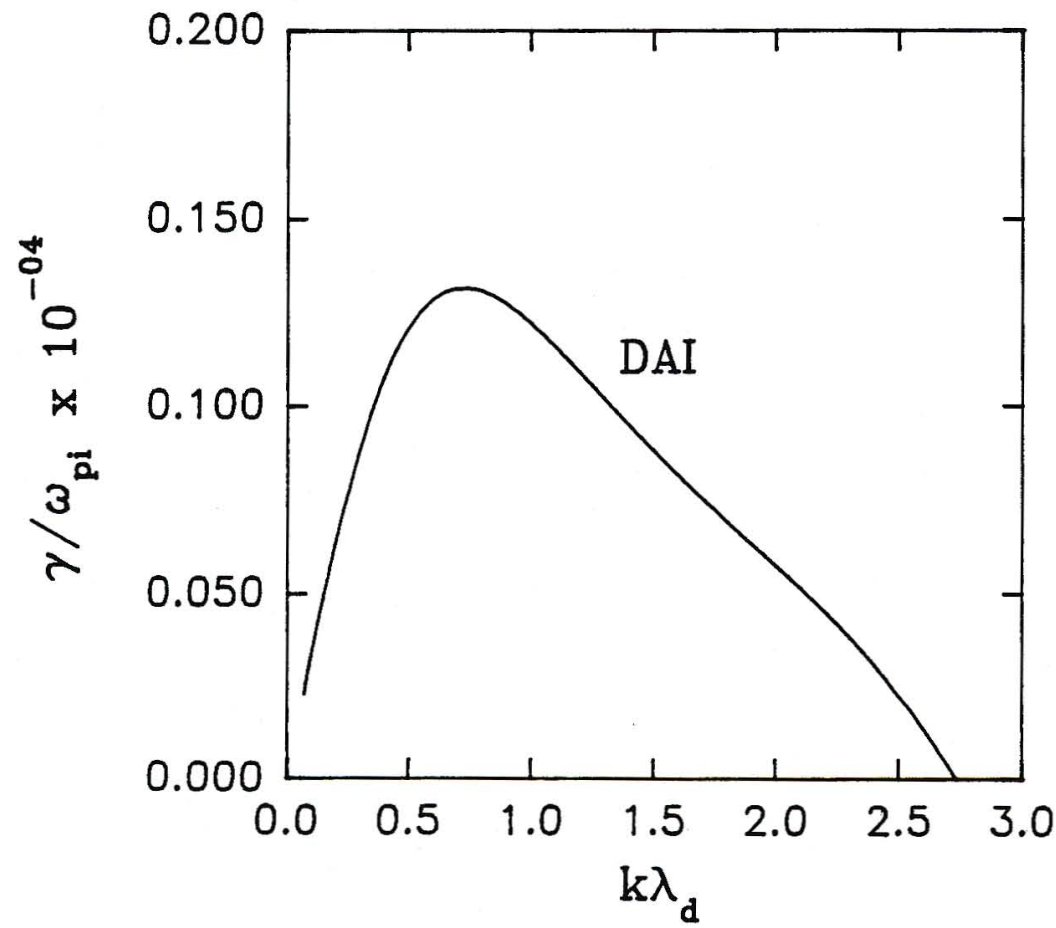

Figure 5.22: Plot of normalized real frequency (1) and growth rate (2) versus $k \lambda_{d}$ for oblique angles of propagation relative to $\mathbf{B}_{\mathbf{o}}$. Here $T_{i}=T_{e}, k_{y} / k=0.95\left(\theta=72^{\circ}\right)$ and $\omega_{p i} / \Omega_{i}=0.01$. All other fixed parameters are as in Figure 5.15 
(1)

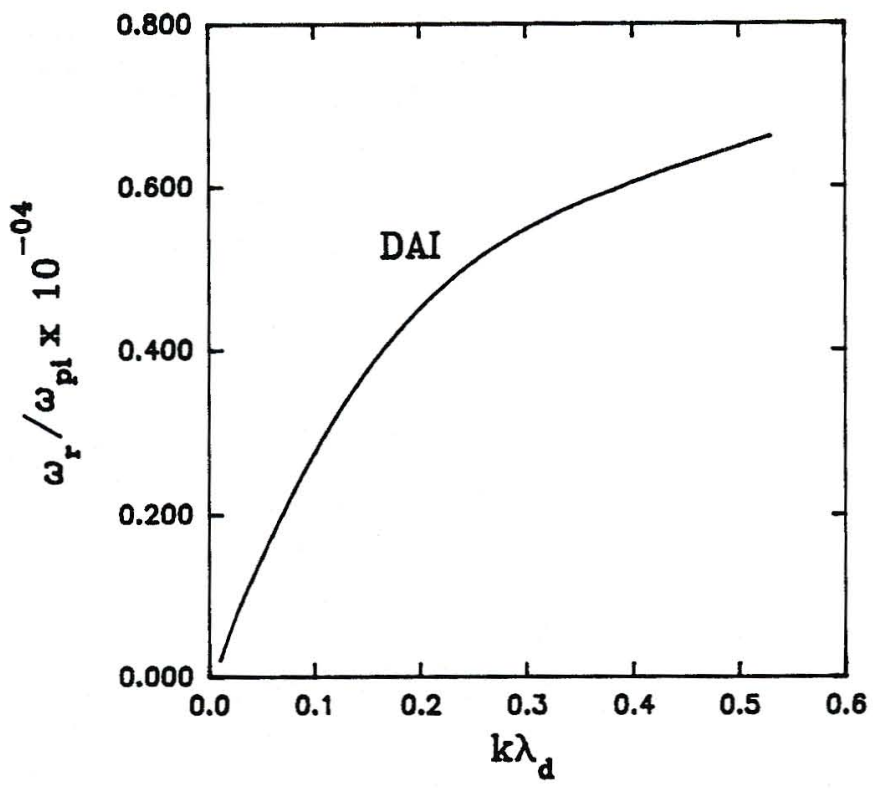

(1)

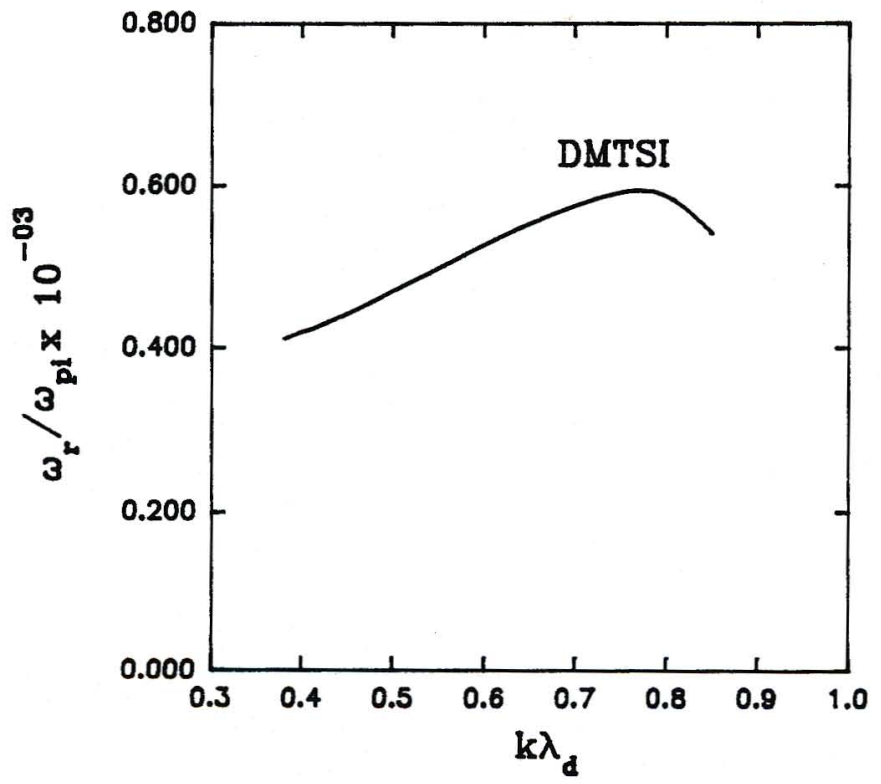

(2)

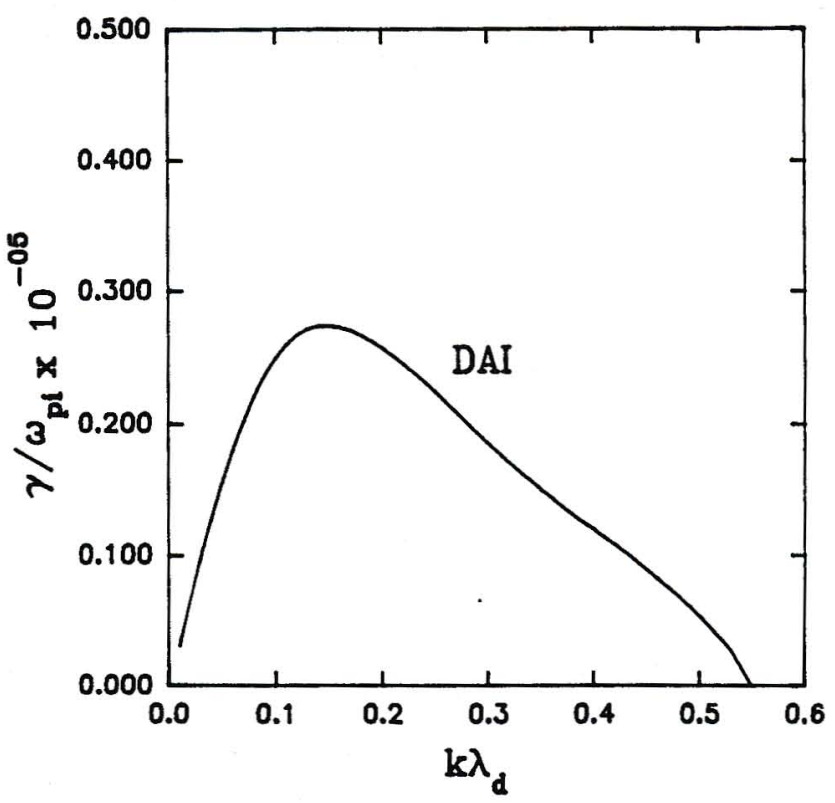

(2)

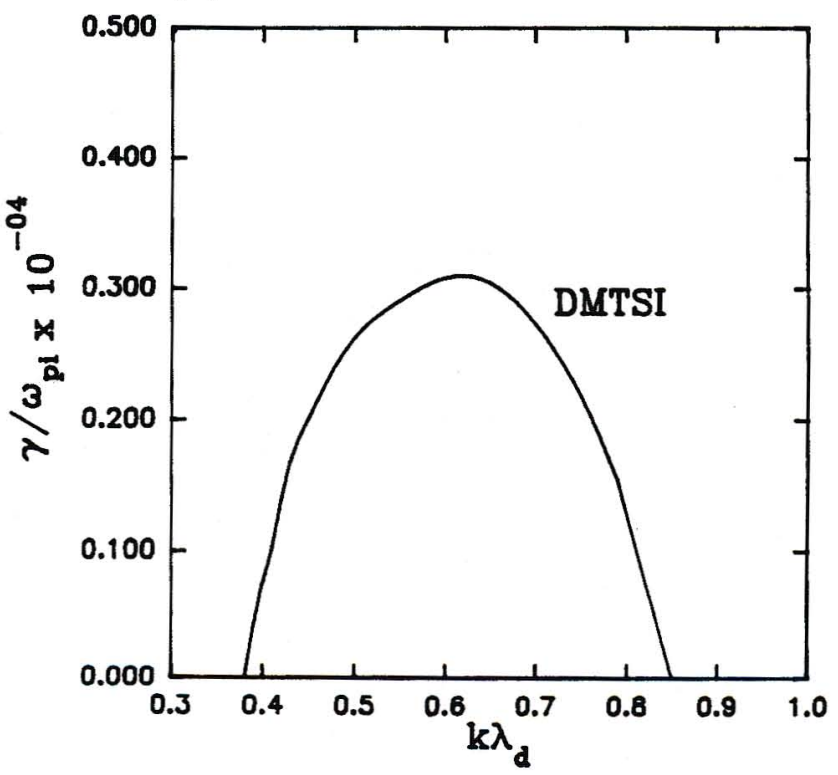

Figure 5.23: Plot of normalized real frequency (1) and growth rate (2) versus $k \lambda_{d}$ for $k_{y} / k=0.95\left(\theta=72^{\circ}\right)$. Here $\omega_{p i} / \Omega_{i}=0.5$. All other fixed parameters are as in Figure 5.15 
(1)

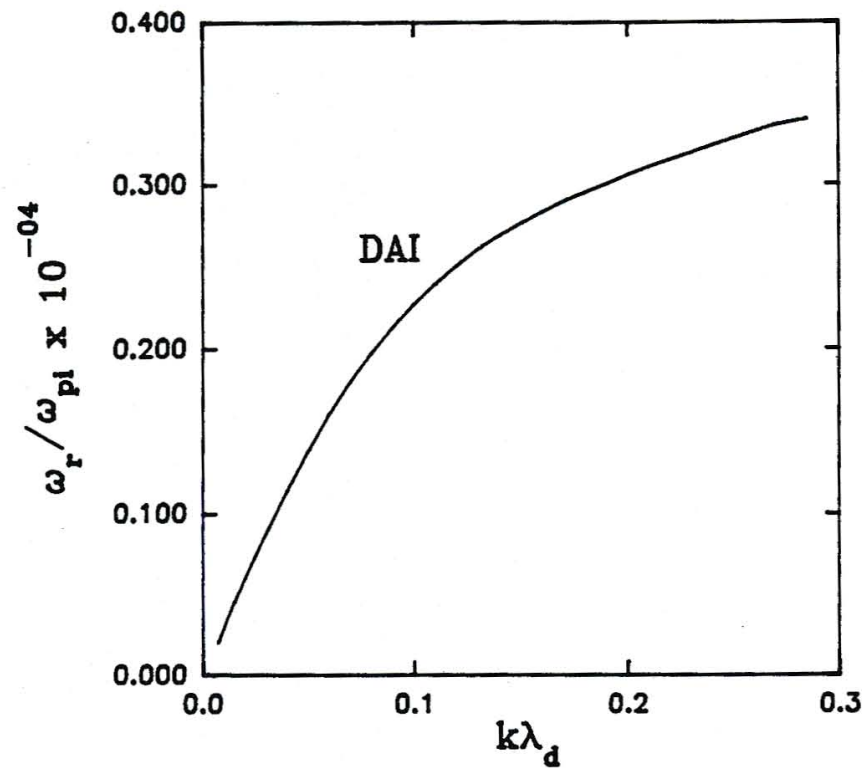

(1)

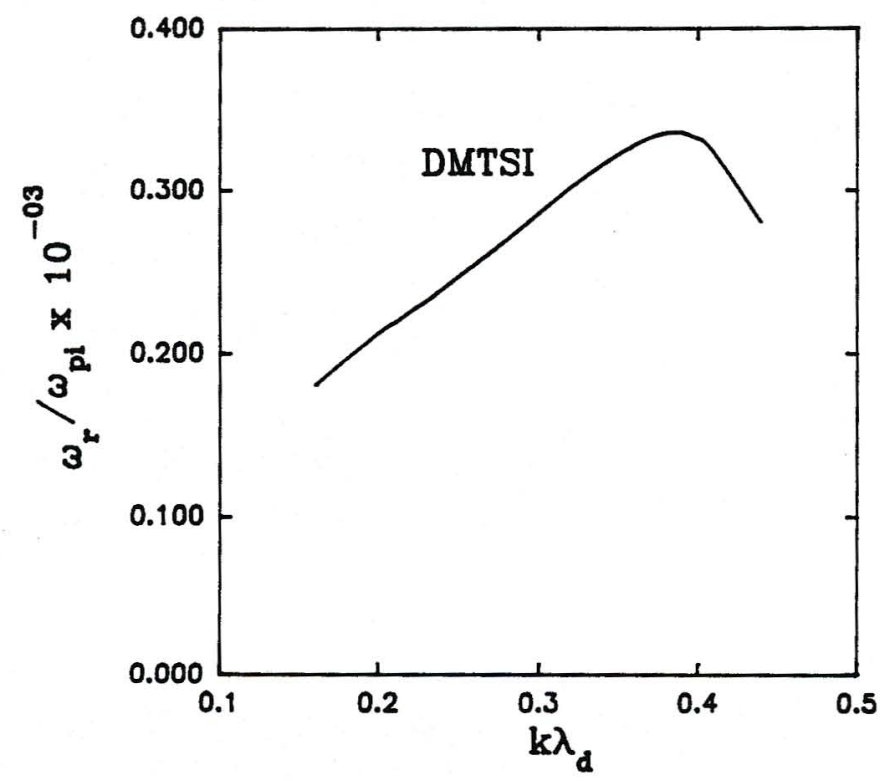

(2)

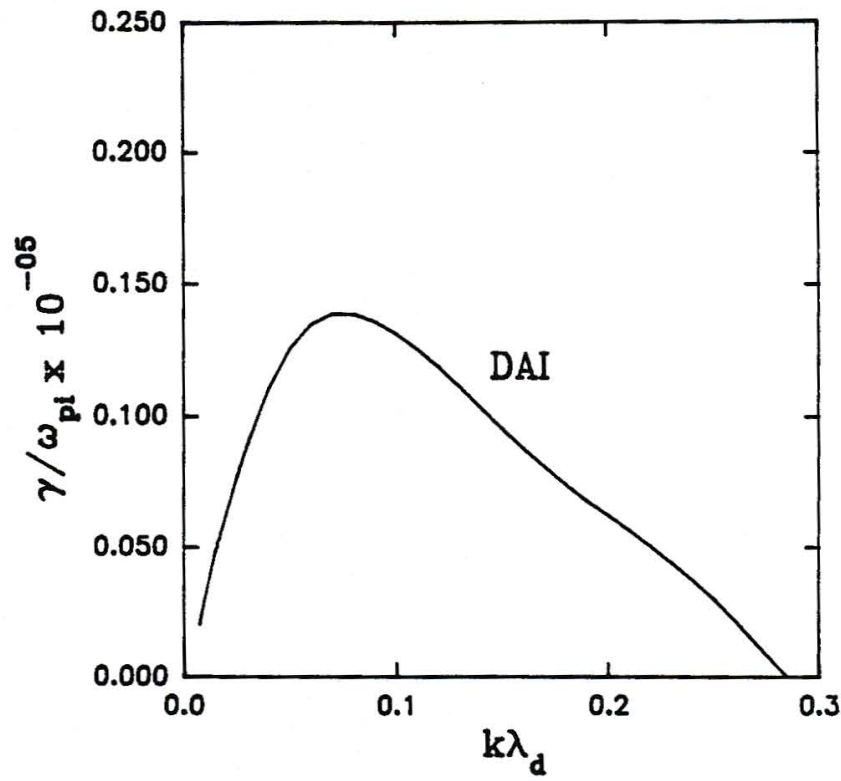

(2)

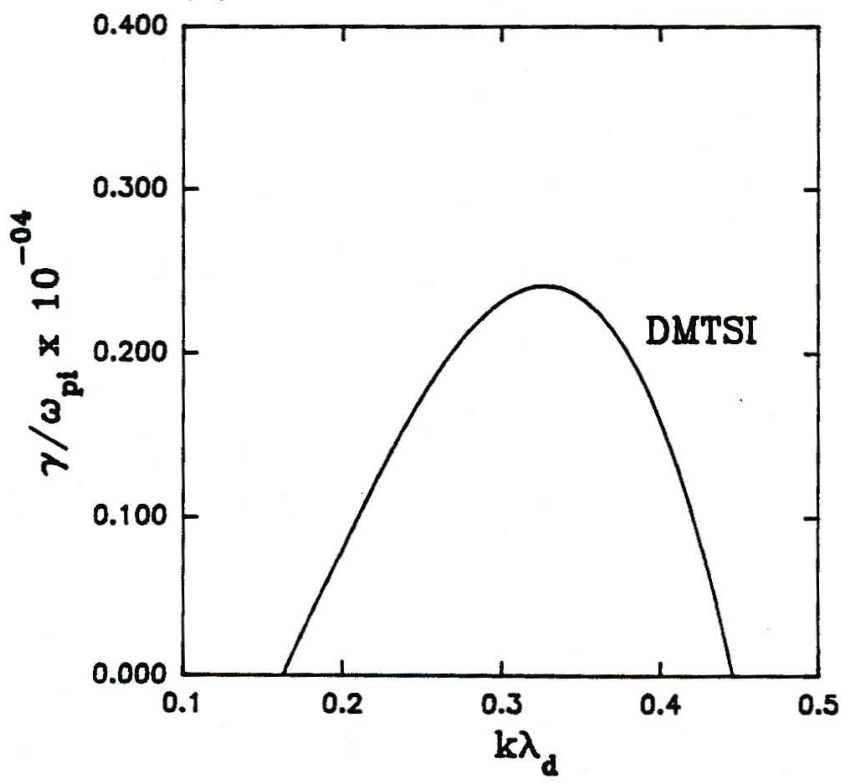

Figure 5.24: Plot of normalized real frequency (1) and growth rate (2) versus $k \lambda_{d}$ for $k_{y} / k=0.95\left(\theta=72^{\circ}\right)$. Here $\omega_{p i} / \Omega_{i}=1.0$. All other fixed parameters are as in Figure 5.15 


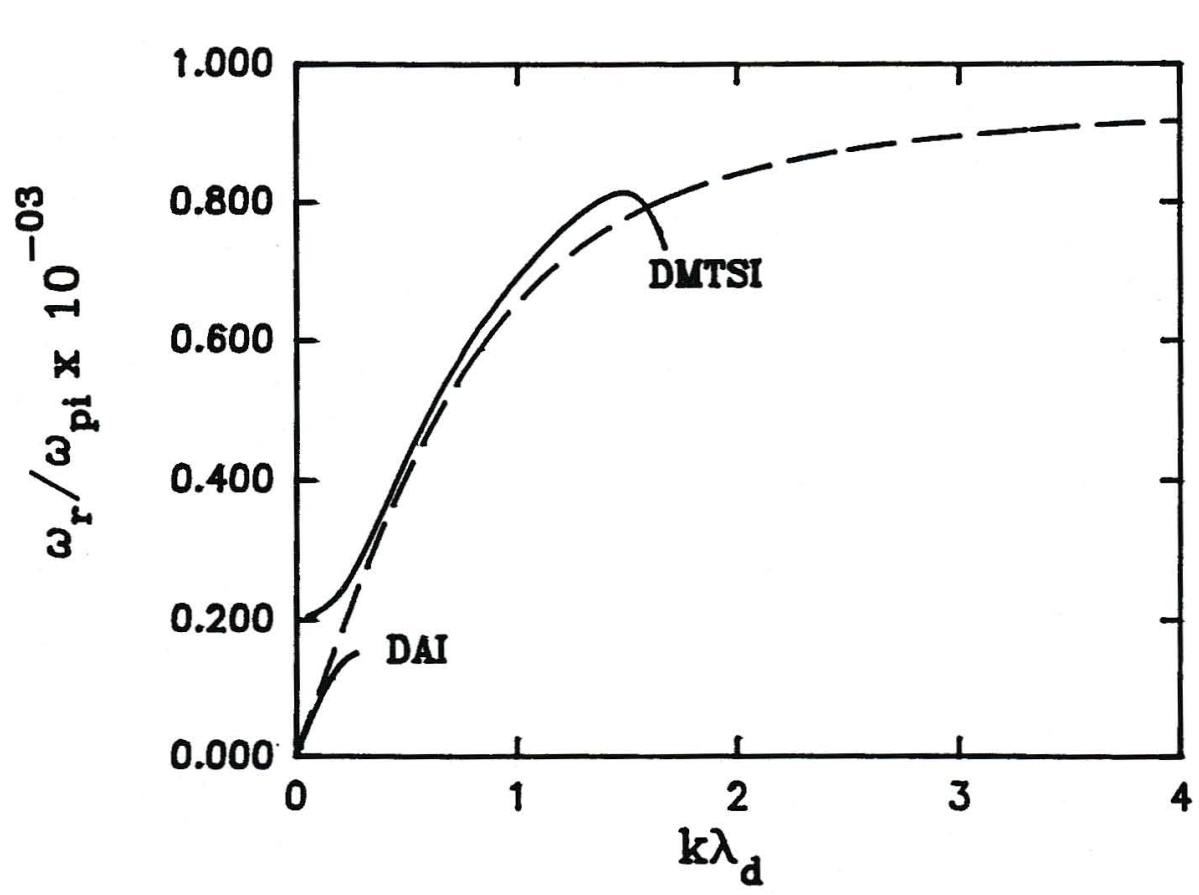

Figure 5.25: Plot of normalized real frequencies as a function of $k \lambda_{d}$ for the two modes in Figure 5.20. The dashed curve corresponds to the approximate solution (5.3). All other fixed parameters are as in Figure 5.20

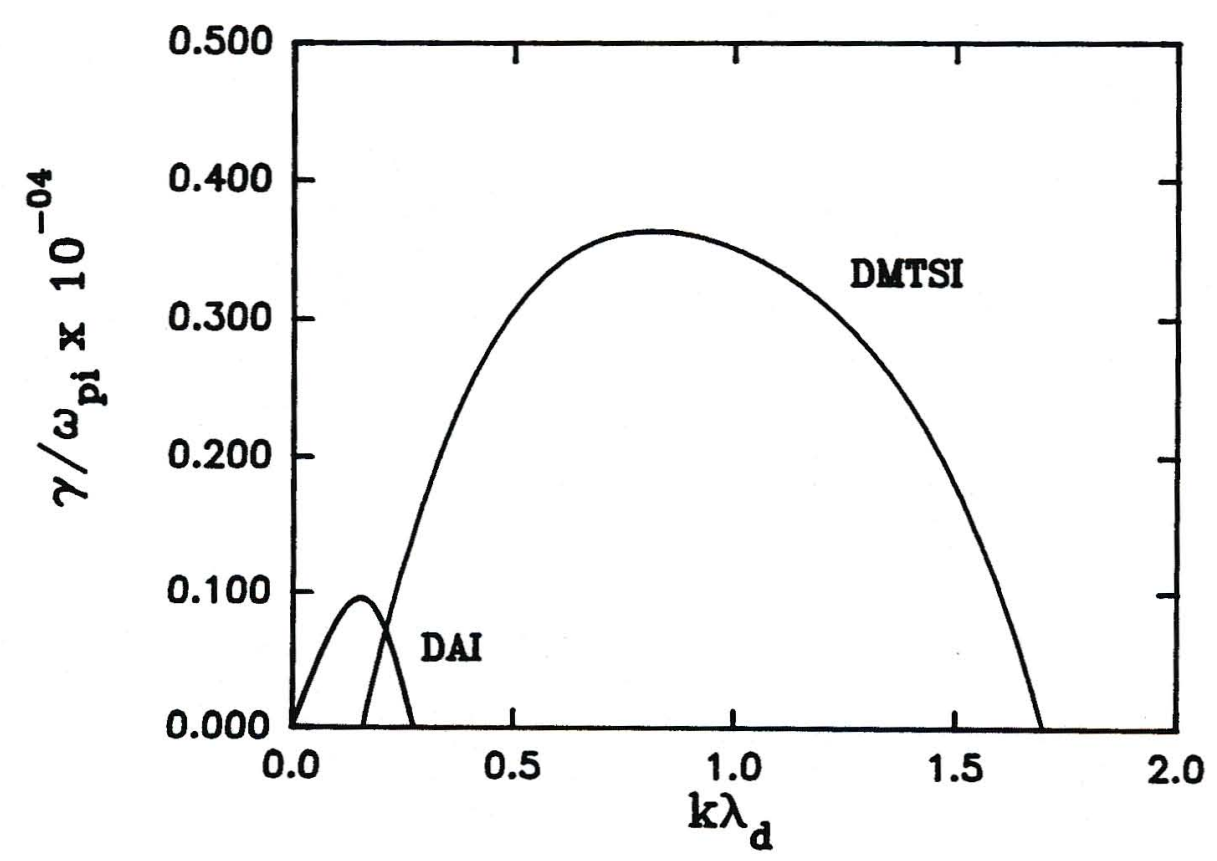

Figure 5.26: Plot of normalized growth rates corresponding to the real frequency curves for the two modes in Figure 5.25 


\section{Chapter 6}

\section{Solution of the Dispersion Relation for Equal Crossfield Drift of Electrons and Ions}

The interest in studying linear instabilities is motivated by the fact that relative drifts between charged particle species provides the necessary energy to excite such instabilities (Havnes,1980,1988; D'Angelo,1990; Bharuthram,1997; Rosenberg et al.,1998). The work that follows in this chapter is an extension of the three-component (one dust species) model of Bharuthram (1997) to a four-component (two dust species) plasma and serves to examine the effect of an additional component of dust on low frequency electrostatic instabilities. We have extended the work of Rosenberg and Krall (1995) to include much wider angles of wave propagation and find that in addition to the dust-modified two-stream instability (DMTSI), the dust-acoustic instability (DAI) could also be excited as first reported by Bharuthram (1997). Results may be applicable to ring plasmas such as Saturn's rings where the magnetized electrons and 
ions have an azimuthal $\mathbf{E} \times \mathbf{B}$ drift relative to the unmagnetized dust grains.

\subsection{The Kinetic Dispersion Relation}

For a four-component plasma comprising of magnetized electrons and ions, and two unmagnetized dust components, the kinetic dispersion relation obtained for electrostatic waves, following the procedure outlined in previous chapters is given by:

$$
\begin{aligned}
0=k^{2} & +\frac{1}{\lambda_{d e}^{2}}\left[1+z_{o e} \sum_{\mathrm{p}=-\infty}^{\infty} Z\left(z_{p e}\right) \Gamma_{p e}\right] \\
& +\frac{1}{\lambda_{d i}^{2}}\left[1+z_{o i} \sum_{\mathrm{p}=-\infty}^{\infty} Z\left(z_{p i}\right) \Gamma_{p i}\right] \\
& +\frac{1}{\lambda_{d d l}^{2}}\left[1+z_{o d l} \sum_{\mathrm{p}=-\infty}^{\infty} Z\left(z_{p d l}\right) \Gamma_{p d l}\right] \\
& +\frac{1}{\lambda_{d d h}^{2}}\left[1+z_{o d h} \sum_{\mathrm{p}=-\infty}^{\infty} Z\left(z_{p d h}\right) \Gamma_{p d h}\right]
\end{aligned}
$$

We recall that $e, i, d l$ and $d h$ represent the electron, ion, light and heavy dust components. Here $z_{p e}=\left(\omega-k_{\perp} V_{E}-\mathrm{p} \Omega_{e}\right) / \sqrt{2} k_{z} C_{e}, z_{p i}=\left(\omega-k_{\perp} V_{E}-\mathrm{p} \Omega_{i}\right) / \sqrt{2} k_{z} C_{i}$, $z_{\text {odl }}=\omega / \sqrt{2} k_{z} C_{d l}$ and $z_{\text {odh }}=\omega / \sqrt{2} k_{z} C_{d h}$.

$\mathbf{V}_{E}$ is the equal $\vec{E}_{o} \times \vec{B}_{o}$ drift of the ions and electrons for an equilibrium electric field $\vec{E}_{o}=-E_{o} \hat{x}$. All other symbols have been defined in previous chapters. Numerical solutions of the full dispersion relation are presented in the next section and are compared with approximate analytical solutions. 


\subsection{Numerical Results}

For simplicity the analysis is restricted to the $y-z$ plane, with $\mathbf{k}=\left(k_{y}, k_{z}\right)=\left(k_{\perp}, k_{z}\right)$. Results are presented in normalized form. The normalizations are the same as in preceding chapters. The quasi-neutrality condition for a two-dust component plasma is given as equation (5.2) in Chapter 5. Parameter values correspond to those in Chapter 5: $T_{e}=T_{i}, T_{d h}=0.01 T_{e}, T_{d l}=0.01 T_{e}, n_{e o}=0.1 n_{o}, Z_{d h}=10^{4}$, $Z_{d l}=10^{2}, m_{d h} / m_{i}=10^{12}, m_{d l} / m_{i}=10^{6}$ and $n_{d l o}=n_{d h o}$ assuming equal densities of the heavy and the light dust species.

Figure 6.1 depicts the normalized instability growth rates as a function of $k_{y} / k=\sin \theta$ for different values of $V_{E}$. Figure 6.2 shows the real frequency curves corresponding to the growth rate curves of Figure 6.1. The high peaks in growth rate in Figure 6.1 for low $k_{y} / k$ correspond to the dust-acoustic instability (DAI) whilst the peaks in the vicinity of $k_{y} / k \approx 1.0$ correspond to the dust-modified two-stream instability (DMTSI) as observed by Bharuthram (1997). This becomes clear in Figure 6.3 by plotting the real frequency and growth rate against normalized wave number $k \lambda_{d}$ for (a) $k_{y} / k=0.175\left(\theta=10^{\circ}\right)$ and (b) $k_{y} / k=0.993\left(\theta=83^{\circ}\right)$. It is evident from Figures 6.1 and 6.2 that for lower drift speeds only the DMTSI is excited but for higher drift velocities the DAI dominates. 


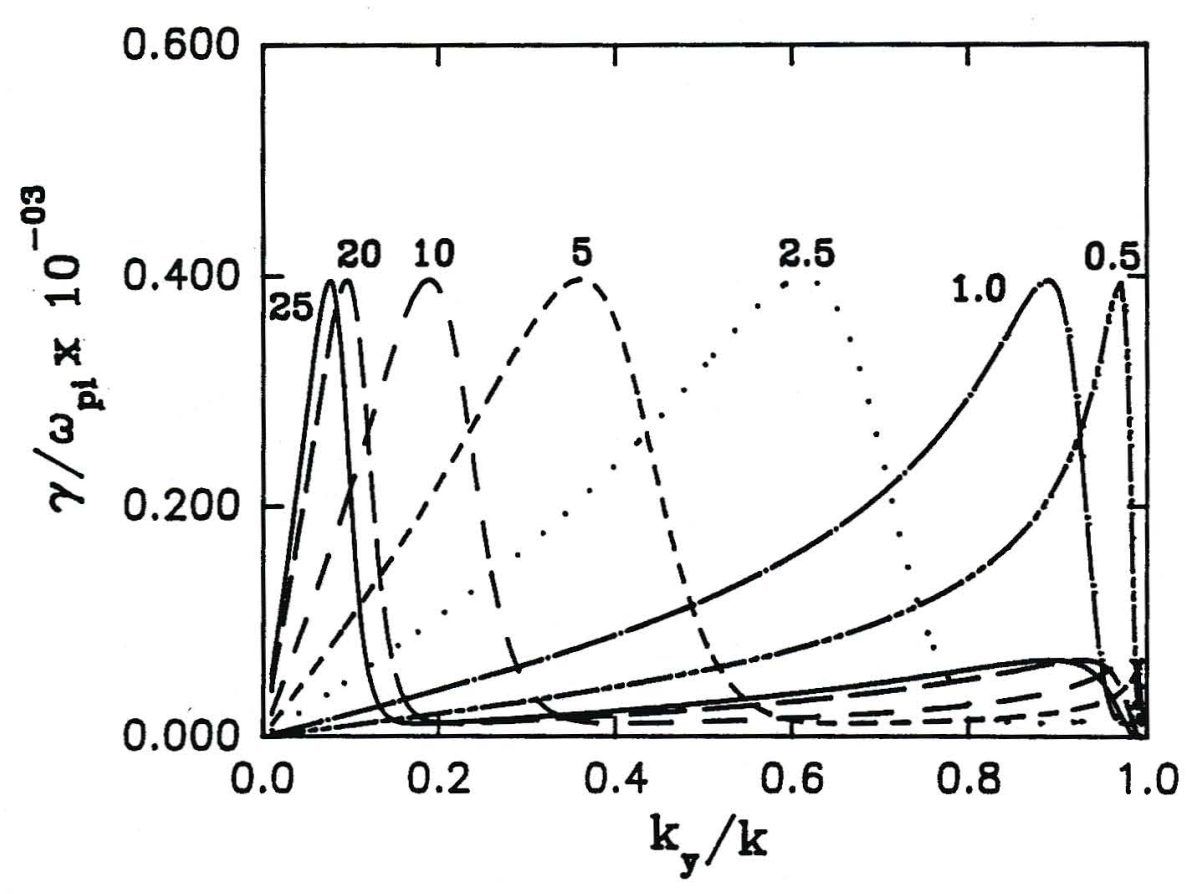

Figure 6.1: Plot of normalized growth rate versus $k_{y} / k(\sin \theta)$. The parameter labelling the curves is the normalized equal ion and electron drift speed $V_{E} / C_{s}$. The fixed parameters are $T_{i}=T_{e}, T_{d l}=0.01 T_{e}, T_{d h}=0.01 T_{e}, n_{e o}=0.1 n_{o}, n_{d l o}=$ $n_{d h o}, Z_{d l}=10^{2}, m_{d l}=10^{6} m_{i}, Z_{d h}=10^{4}, m_{d h}=10^{12} m_{i}, k \lambda_{d}=0.7$ and $\omega_{p i}=$ $0.01 \omega_{c i}$

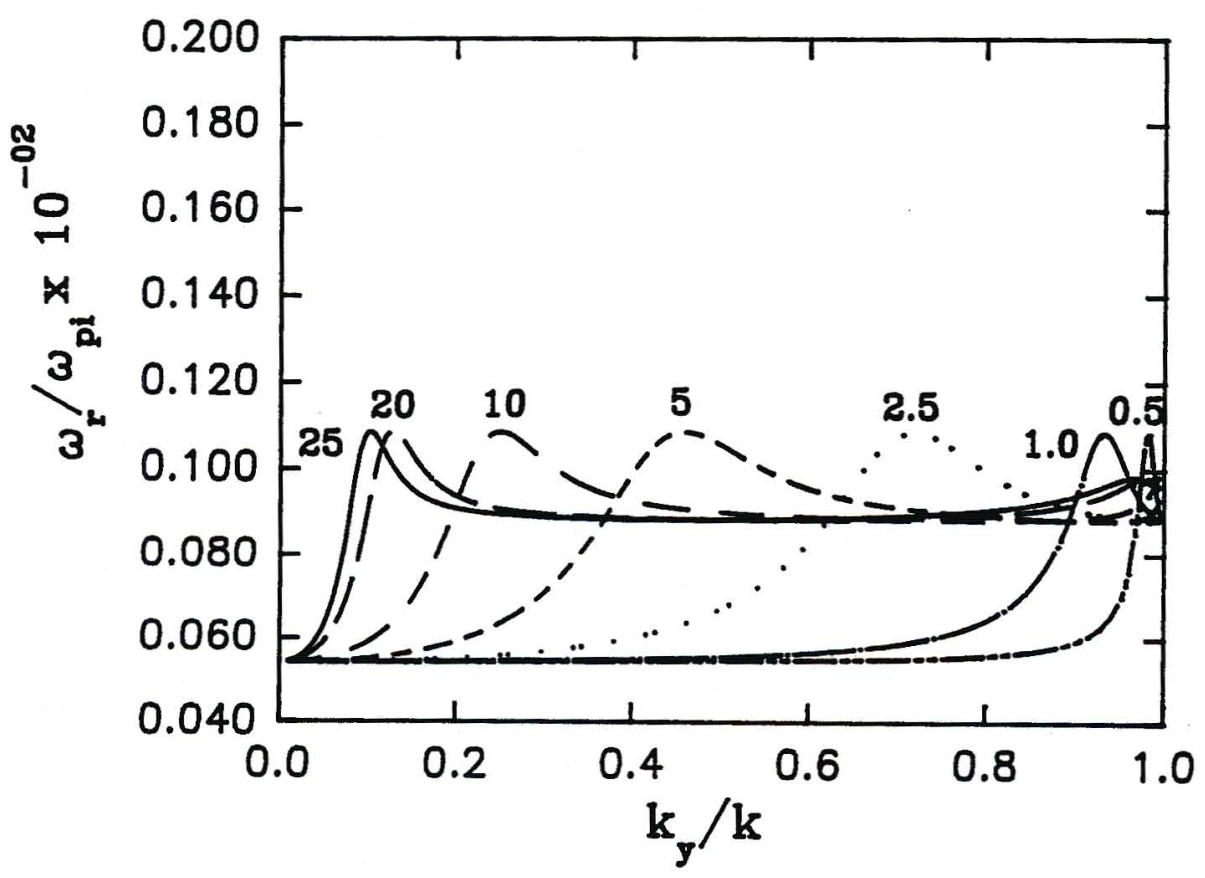

Figure 6.2: Plot of normalized real frequencies corresponding to the growth rate curves of Figure 6.1. The parameter labelling the curves is $V_{E} / C_{s}$ 
The numerical real frequency in Figure 6.3 is compared with the approximate analytical solution of the dispersion relation for a four-component plasma (two dust constituents), equation (5.3) in Chapter 5:

$$
\omega_{r}^{2}=k^{2} C_{s}^{2} \frac{\left\{\frac{m_{i}}{m_{d l}} Z_{d l}\left(\delta-1-Z_{d h} \frac{n_{d h e}}{n_{e o}}\right)+\frac{m_{i}}{m_{d h}} Z_{d h}\left(\delta-1-Z_{d l} \frac{n_{d l o}}{n_{e o}}\right)\right\}}{\left(1+\delta \frac{T_{e}}{T_{i}}+\delta k^{2} \lambda_{d}^{2}\right)}
$$

Comparison is also made in Figure 6.4 of the numerical solution for the real frequency of the dispersion relation for a three-component plasma (single dust constituent) with the approximate solution, equation (4.3) in Chapter 4:

$$
\omega_{r}^{2}=k^{2} C_{s}^{2} Z_{d}(\delta-1) \frac{m_{i}}{m_{d}} \frac{1}{\left(1+\delta T_{e} / T_{i}+\delta k^{2} \lambda_{d}^{2}\right)}
$$

derived by Rosenberg (1993) for the dust-acoustic mode. The numerical solutions for the normalized real frequency and growth rate (for wave propagation almost perpendicular to $\mathbf{B}_{\mathbf{o}}$ ) in Figures 6.3(b) and 6.4(b) correspond to the DMTSI of Rosenberg and Krall (1995).

Figure 6.5 demonstrates the effect of varying the equilibrium electron density on the instability growth rate for fixed $n_{d h o}=n_{d l o}$. It is seen that for the DAI, the maximum growth rate $\gamma_{\max }$ decreases as the equilibrium electron density $n_{e o}$ increases. This behaviour was also observed by Bharuthram (1997) for a single dust species plasma and for the ion-acoustic and EIC modes seen by D'Angelo $(1990,1995)$. The opposite is found to be true for the DMTSI which is consistent with the findings of Bharuthram (1997). The DAI will therefore dominate in dusty plasmas with small electron concentrations whereas large electron concentrations are needed for the DMTSI to be excited. 
(a)

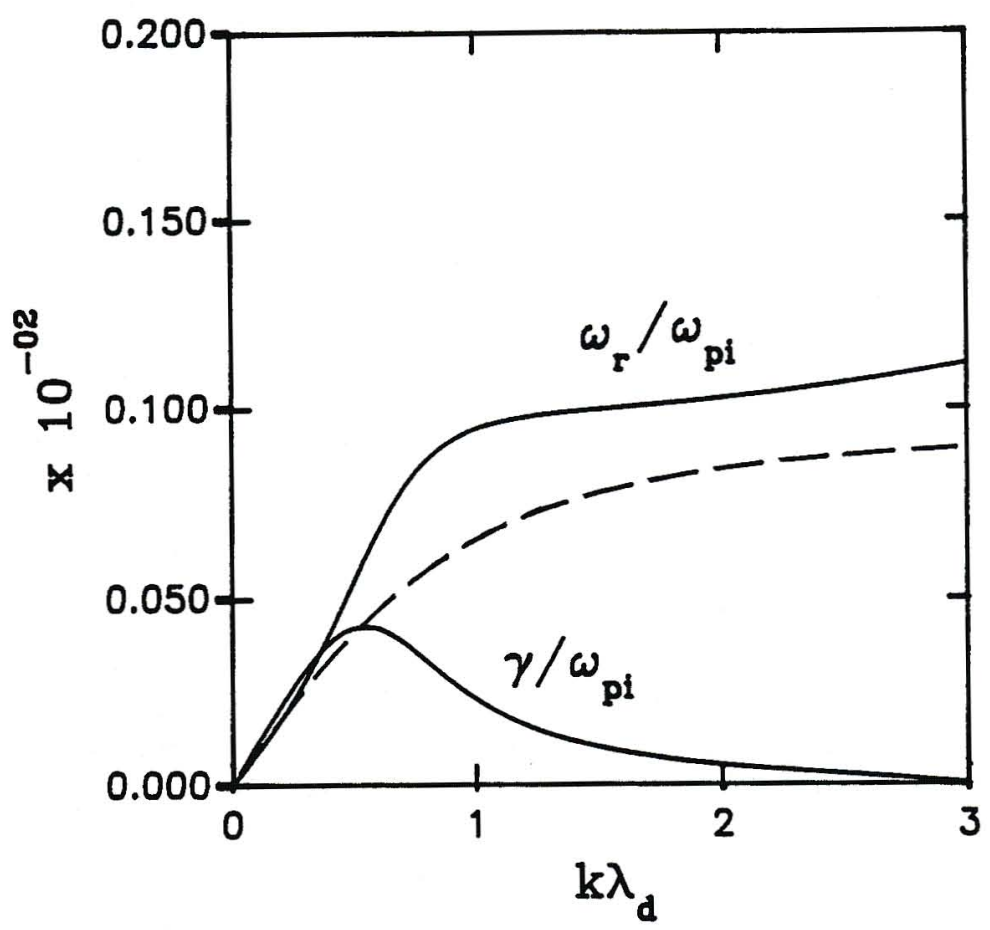

(b)

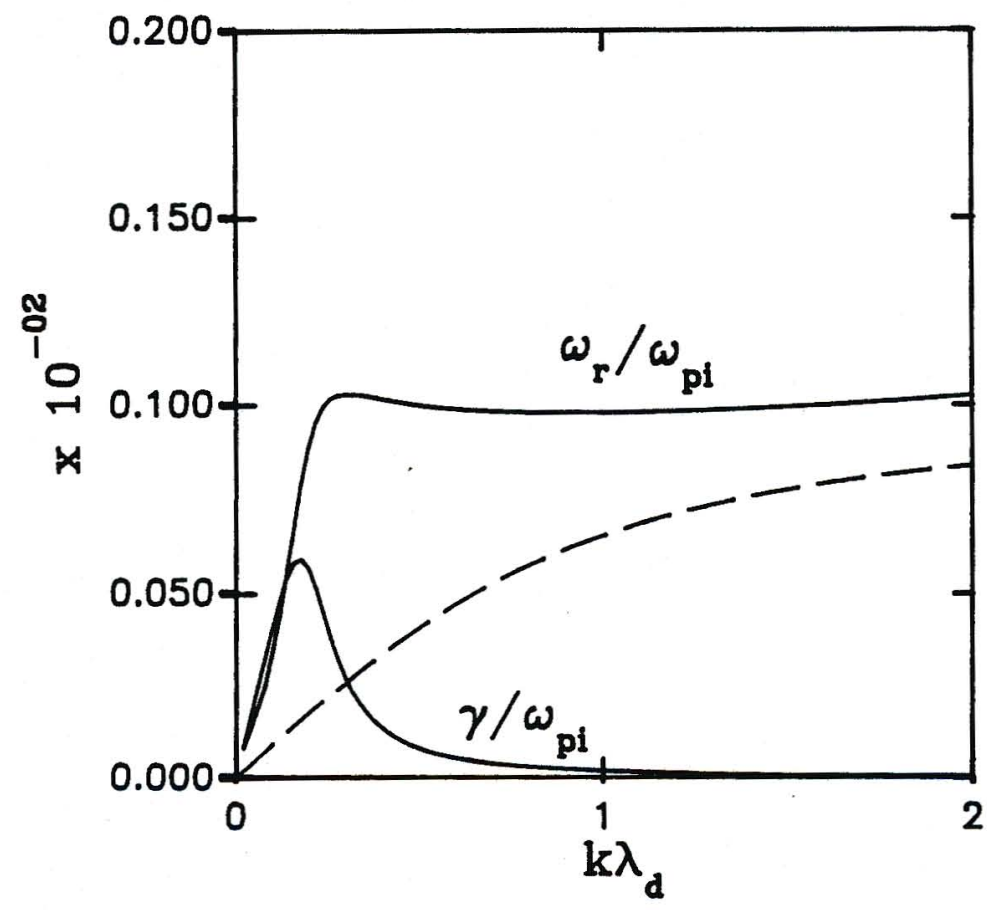

Figure 6.3: Plot of the normalized real frequency and growth rate versus $k \lambda_{d}$ for (a) $k_{y} / k=0.175\left(\theta=10^{\circ}\right)$ and (b) $k_{y} / k=0.993\left(\theta=83^{\circ}\right)$. Here $V_{E}=10 C_{s}$. The broken line is the result calculated from the approximate solution (6.2). All other fixed parameters are as in Figure 6.1 
(a)

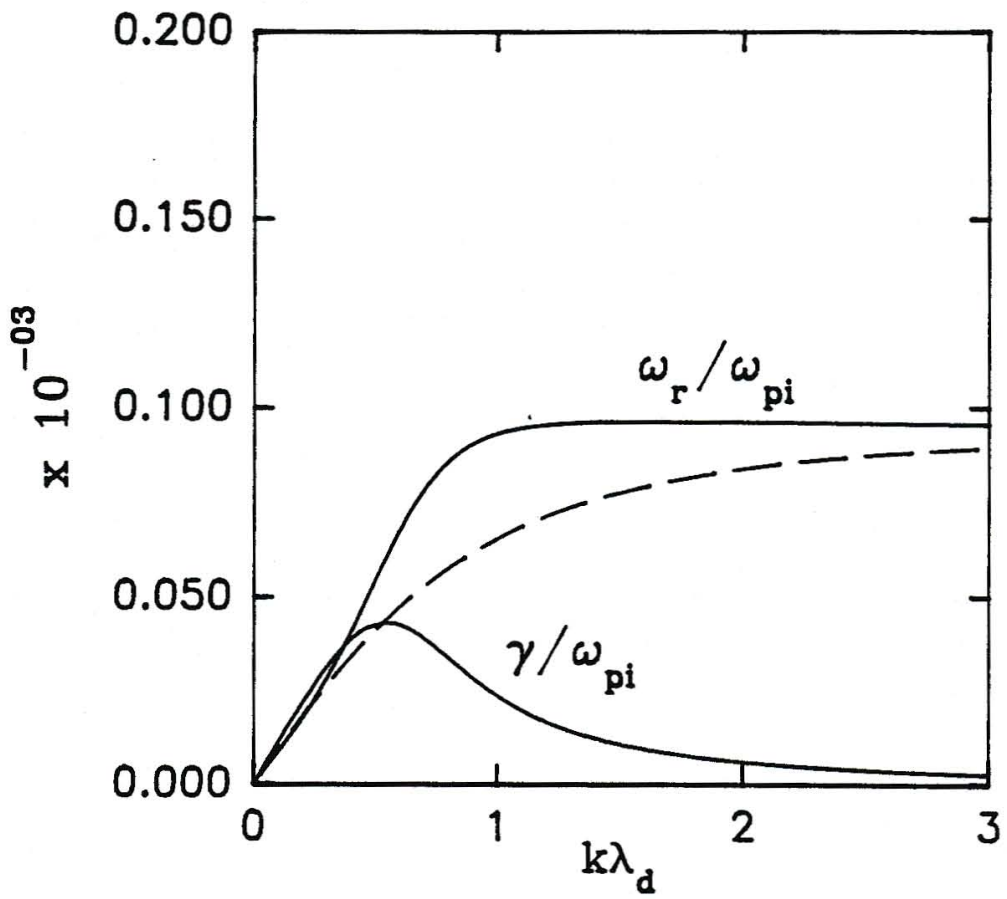

(b)

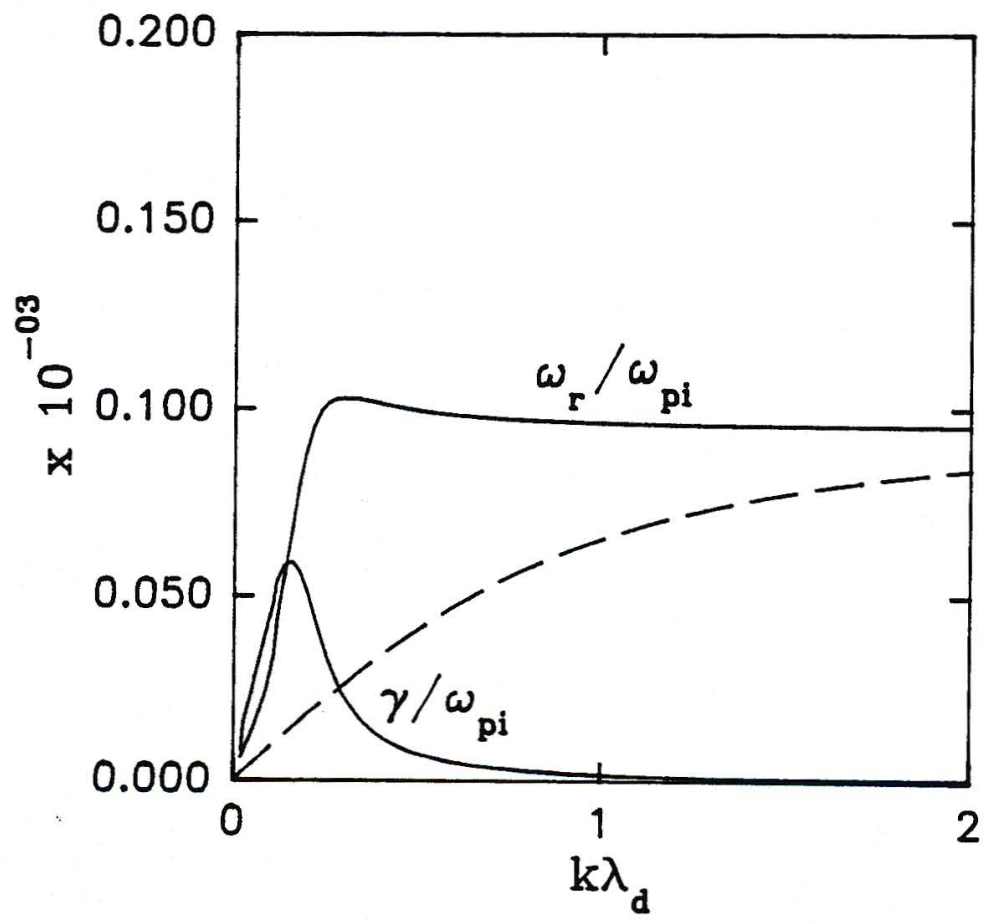

Figure 6.4: Plot of the normalized real frequency and growth rate versus $k \lambda_{d}$ for (a) $k_{y} / k=0.175\left(\theta=10^{\circ}\right)$ and (b) $k_{y} / k=0.993\left(\theta=83^{\circ}\right)$. Here $m_{d}=10^{12} m_{i}$ and $Z_{d}=10^{4}$ for a three-component (single heavy dust species) plasma. The broken line is the result calculated from the approximate solution (6.3). All other fixed parameters are as in Figure 6.3 


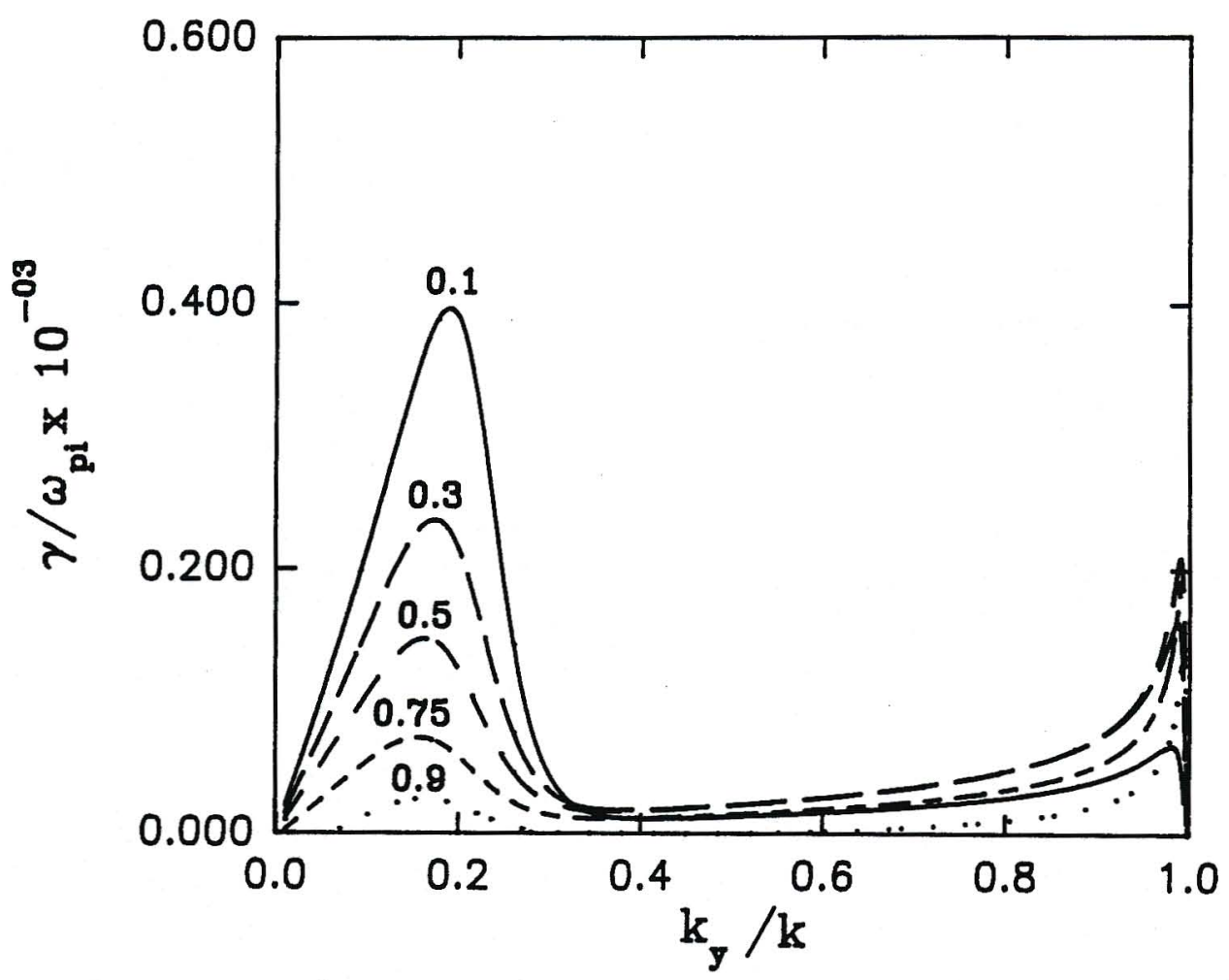

Figure 6.5: Plot of the normalized growth rate versus $k_{y} / k$ for $V_{E}=10 C_{s}$. The parameter labelling the curves is the normalized equilibrium electron density $n_{e o} / n_{\circ}$. All other fixed parameters are as in Figure 6.1 
Figure 6.6 shows the effect of varying the normalized ion temperature $T_{i} / T_{e}$ on the growth rate of the instability. The lowering of the growth rate with an increase in $T_{i} / T_{e}$ is due to the 'flattening' of the ion velocity distribution as explained in preceding chapters.

Figure 6.7 shows the effect of varying the ratio of the light relative to the heavy dust densities $\left(n_{d l o} / n_{d h o}\right)$ on the growth rate of the instability. The equilibrium electron density is fixed at $n_{e o}=0.1 n_{o}$. The corresponding real frequencies are shown in Figure 6.8. It is seen that as $n_{d l o} / n_{d h o}$ decreases ( $n_{d h o}$ increasing), the growth rate and real frequency of the DAI decreases due to the instability becoming dominated by the heavier species and hence the slower inertial response of the heavier particles to perturbations in potential. For $n_{d l o}<0.01 n_{d h o}$, the growth rate becomes comparatively very small. Thus when the fraction of the light dust grains is very small, the instability is present on the 'heavy dust fluid' time scale with growth rates two orders of magnitude lower than those associated with the light dust grains. We find that the growth rate of the DMTSI also decreases as the concentration of the heavy dust grains starts increasing. 
Following a study of the relevant literature (Chapter 2) and the derivation of the kinetic dispersion relation (Chapter 3), Chapter 4 presents a study of the dustacoustic instability in a three-component plasma comprising of ions, electrons and a single heavy dust component. The instability is driven by a drift of the ions in the direction of the external magnetic field $\mathbf{B}_{\mathbf{0}}$. The effect of the variation of parameters such as ion drift speed, densities, ion temperature and magnetic field strength on the real frequency and growth rate of the instability is studied. In this chapter the instability is also studied in a three-component plasma comprising of lighter dust grains. The real frequency and growth rates associated with the light dust constituent are found to be two orders of magnitude higher than those associated with the heavier dust grains. This is to be expected since perturbations in potential would occur on smaller time scales than those associated with the heavier, less mobile dust grains. The numerical solution of the full dispersion relation is found to be in excellent agreement with the approximate analytical solution.

In Chapter 5 the instability is investigated in a four-component plasma comprising of both the light and heavy dust constituents of Chapter 4 . In addition to the variation of parameters undertaken in Chapter 4, the effect of varying the density ratio of the light relative to the heavy dust grains $\left(n_{d l o} / n_{d h o}\right)$, is also studied. The transition from the dust-acoustic instability corresponding to the single dust constituent (threecomponent) plasma of the heavy to that of the light dust grains is continuous and occurs over an extremely large range of $n_{d l o} / n_{d h o}$. Assuming equal concentrations of the light and the heavy dust constituents, we observe that the real frequency lies 
intermediate to those corresponding to the light and heavy dust components of the three-component plasmas of Chapter 4. The numerical solution of the full dispersion relation agrees well with the approximate solution for smaller values of normalized wave number $k \lambda_{d}$.

The effects of the parameter variation in Chapters 4 and 5 may be summarized as follows: An increase in the ion drift speed ( $V_{o}$ increasing) enhances the instability growth rate as more free energy becomes available to drive the instability. An increase in the ion temperature $T_{i}$ results in a lowering of the growth rates. This is attributed to a 'flattening' of the ion velocity distribution. It is also found that the dust-acoustic instability is more easily excited in plasmas with small electron concentrations due to a reduction in the Landau damping. An increase in the magnetic field strength $B_{o}$ enhances wave growth and is attributed to the effect of the increase in particle magnetization. As expected, maximum growth is obtained for propagation along $\vec{B}_{o}$.

In Chapter 6 we modify the model of Chapter 5 and consider an equal $\mathbf{E} \times \mathbf{B}_{\mathbf{o}}$ drift of the magnetized ions and electrons relative to the unmagnetized dust grains. This model is relevant to the planetary rings of Saturn where the unmagnetized, levitated dust grains are stationary, while the magnetized electrons and ions have an azimuthal $\vec{E} \times \vec{B}_{o}$ drift (Rosenberg,1993). The mass and charge of the two dust components are the same as in Chapter 5. The effect of varying parameters such as drift speeds, particle densities and normalized ion temperature on the real frequency and growth rate is examined. We find that the DAI dominates for small angles of wave propagation relative to $\vec{B}_{o}$, whereas for almost perpendicular propagation, the DMTSI is excited. 
The DAI is more easily excited in plasmas with small electron concentrations which is consistent with the findings in Chapters 4 and 5. The numerical solution for the real frequency $\omega_{r}$ of the full dispersion relation is compared with the approximate solution.

There are several extensions of the work in this thesis. Charge variation effects of the dust particles which could play an important role, should be incorporated into our model. The number of dust components in our model is limited to just two. A real dusty plasma comprises of an extremely diverse range of grain sizes. We could generalize our model to include a variable number $n$ of different (with respect to mass and charge) dust constituents and employ a grain size distribution for the dust grains, due to the grain size generally being distributed according to a power law distribution (Meuris et al.,1997). 


\section{References}

[1] Barkan, A, D'Angelo, N and Merlino, R.L., Planet. Space Sci. 43, 905, 1995.

[2] Barkan, A, Merlino, R.L. and D'Angelo, N, Phys. Plasmas 2, 3563, 1995.

[3] Bharuthram, R., Planet. Space Sci. 45, 379, 1997.

[4] Bharuthram, R., Saleem, H. and Shukla, P.K., Phys. Scr. 45, 512, 1992.

[5] Bharuthram, R. and Shukla, P.K., Planet. Space Sci. 40, 465, 1992.

[6] Bharuthram, R. and Pather, T., Planet. Space Sci. 44, 137, 1996.

[7] Bharuthram, R. and Rosenberg, M., Planet. Space Sci. 46, 425, 1998.

[8] Chen, F.F., Introduction to Plasma Physics and Controlled Fusion, Plenum, New York, 1984.

[9] Chow, V.W. and Rosenberg, M., Planet. Space Sci. 43, 613, 1995.

[10] Chow, V.W. and Rosenberg, M., Phys. Plasmas 3, 1202, 1996.

[11] D'Angelo, N, Planet. Space Sci. 38, 1143, 1990.

[12] D'Angelo, N, Experiments on dust-plasma interactions, The Sixth Workshop on the Physics of Dusty Plasmas, University of California, San Diego, 22-24 March 1995.

[13] De Angelis, U., Formisano, V and Giordano, M., J. Plasma Phys. 40, 399, 1988. 
[14] Fried, B.D. and Conte, S.D., The Plasma Dispersion Function, Academic Press, New York, 1961.

[15] Gary, S.P and Sanderson, J.J., J. Plasma Phys. 4, 739, 1970.

[16] Goertz, C.K., Rev. Geophys. 27, 271, 1989.

[17] Havnes, O., Astron. Astrophys. 90, 106, 1980.

[18] Havnes, O., Astron. Astrophys. 193, 309, 1988.

[19] Lakhina, G.S., Phys. Scr. T50, 114, 1994.

[20] Merlino, R.L., Barkan, A.,Thompson, C. and D'Angelo, N., Phys. Plasmas 5, $1607,1998$.

[21] Meuris, P., Planet. Space Sci. 45, 1171, 1997.

[22] Meuris, P., Verheest, F. and Lakhina, G.S., Planet. Space Sci. 45, 449, 1997.

[23] Northrop, T.G., Phys. Scr. 45, 475, 1992.

[24] Prabhakara, H.R. and Tanna, V.L., Phys. Plasmas 3, 3176, 1996.

[25] Rao, N.N., Shukla, P.K. and Yu, M.Y., Planet. Space Sci. 38, 543, 1990.

[26] Robertson, S., Phys. Plasmas 2, 2200, 1995.

[27] Rosenberg, M., Planet. Space Sci. 41, 229, 1993.

[28] Rosenberg, M. and Krall, N.A., Planet. Space Sci. 42, 889, 1994. 
[29] Rosenberg, M. and Krall, N.A., Planet. Space Sci. 43, 619, 1995.

[30] Rosenberg, M. and Krall, N.A., Phys. Plasmas 3, 644, 1996.

[31] Rosenberg, M. and Chow, V.W., Planet. Space Sci. 46, 103, 1998.

[32] Shukla, P.K., Phys. Scr. 45, 504, 1992.

[33] Shukla, P.K. and Silin, V.P., Phys. Scr. 45, 508, 1992.

[34] Singh, P., University of Durban-Westville, M.Sc. Thesis, 1989.

[35] Thomas, H., Morfill, G.E., Demmel, V., Goree, J., Feuerbacher, B. and Mohlmann, D., Phys. Rev. Lett 73, 652, 1994.

[36] Verheest, F., Space Sci. Rev. 77, 267, 1996.

[37] Watson, G., A Treatise on the Theory of Bessel Functions, Cambridge University Press, Second Edition, 1944.

[38] Whipple, E.C., Northrop, T.G. and Mendis, D.A., J. Geophys. Res. 90, 7405, 1985. 